\title{
Witten index and wall crossing
}

\section{Kentaro Hori, ${ }^{a}$ Heeyeon $\mathrm{Kim}^{b}$ and Piljin $\mathrm{Yi}^{c}$}

${ }^{a}$ Kavli Institute for the Physics and Mathematics of the Universe (WPI), University of Tokyo, Kashiwa, Chiba 27\%-8583, Japan

${ }^{b}$ Department of Physics and Astronomy, Seoul National University, Seoul 151-147, South Korea

${ }^{c}$ School of Physics, Korea Institute for Advanced Study, Seoul 130-722, South Korea

E-mail: kentaro.hori@ipmu.jp, hykim@phya.snu.ac.kr, piljin@kias.re.kr

ABSTRACT: We compute the Witten index of one-dimensional gauged linear sigma models with at least $\mathcal{N}=2$ supersymmetry. In the phase where the gauge group is broken to a finite group, the index is expressed as a certain residue integral. It is subject to a change as the Fayet-Iliopoulos parameter is varied through the phase boundaries. The wall crossing formula is expressed as an integral at infinity of the Coulomb branch. The result is applied to many examples, including quiver quantum mechanics that is relevant for BPS states in $d=4 \mathcal{N}=2$ theories.

KeYwords: Supersymmetric gauge theory, Field Theories in Lower Dimensions, D-branes ARXIV EPRINT: 1407.2567 


\section{Contents}

1 Introduction 1

2 Gauged linear sigma models in one dimension $\quad 6$

2.1 The models 6

$\begin{array}{lll}2.2 & \text { The index } & 14\end{array}$

$\begin{array}{lll}2.3 \text { Phases } & 16\end{array}$

2.4 Examples 21

3 Coulomb branch $\quad 28$

3.1 The outline 28

3.2 The $\mathrm{CP}^{N-1}$ model 33

$\begin{array}{lll}3.3 & \text { General U(1) theory } & 37\end{array}$

$\begin{array}{ll}3.4 & \text { Simple wall crossing }\end{array}$

4 The index $\quad 41$

$\begin{array}{ll}4.1 & \text { Setting up the computation }\end{array}$

4.2 One loop integral 44

$\begin{array}{lll}4.3 & \mathrm{U}(1) \text { theories } & 47\end{array}$

4.4 Scaling limits 50

4.5 Higher rank theories $\quad 52$

4.6 The $D$-contour $\quad 55$

$\begin{array}{lll}4.7 & \text { A systematic procedure } & 57\end{array}$

$\begin{array}{ll}4.8 \text { The result } & 59\end{array}$

$\begin{array}{llr}5 & \text { A wall crossing formula } & \mathbf{6 0}\end{array}$

$\begin{array}{lll}5.1 \mathrm{U}(1) \text { theories } & 61\end{array}$

$\begin{array}{lll}5.2 & \text { General Abelian theory } & 62\end{array}$

5.3 Simple wall crossing in a non-Abelian theory 64

6 Systems with $\mathcal{N}=4$ supersymmetry $\quad 66$

$\begin{array}{lll}6.1 \text { Grassmannian } & 66\end{array}$

$\begin{array}{lll}6.2 & \text { Hypersurface in projective space } & 67\end{array}$

$\begin{array}{lll}6.3 & \text { Flavor decoupling in compact models } & 69\end{array}$

$\begin{array}{lll}\text { 6.4 Flavor non-decoupling in non-compact models } & 71\end{array}$

$\begin{array}{ll}\text { 6.5 "The two parameter model" } & 73\end{array}$

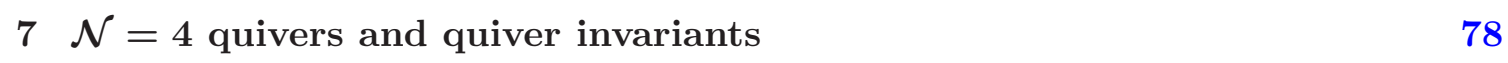

$\begin{array}{lll}7.1 & \text { Abelian }(k+1) \text {-gon } & 79\end{array}$

7.2 Non-Abelian triangle quivers of ranks $(k, 1,1) \quad 80$

7.3 Quiver invariants 84 
8 Systems with $\mathcal{N}=2$ supersymmetry

8.1 The $\mathrm{CP}^{N-1}$ model $\quad 88$

$\begin{array}{lll}8.2 \text { Grassmannian } & 89\end{array}$

$\begin{array}{lll}8.3 & \text { Distler-Kachru model } & 90\end{array}$

$\begin{array}{ll}8.4 & \text { Triangle quiver }\end{array}$

$\begin{array}{ll}\text { A } 1 \mathrm{~d} \mathcal{N}=\mathbf{2} \text { supersymmetry } & \mathbf{9 8}\end{array}$

$\begin{array}{ll}\text { A.1 } 1 \mathrm{~d} \mathcal{N}=2 \text { superspace } & 98\end{array}$

$\begin{array}{llr}\text { A.2 Gauge theory } & 99\end{array}$

$\begin{array}{lll}\text { A.3 Non-linear sigma model } & 101\end{array}$

$\begin{array}{ll}\text { A.4 } \mathcal{N}=4 \text { theories } & 101\end{array}$

\section{Introduction}

The present paper is about Witten index [1] of one dimensional gauge theories with at least $\mathcal{N}=2$ supersymmetry. We do not attempt to consider the most general gauge theories but restrict our target to a class of theories called "gauged linear sigma models". A theory in this class has gauge group with at least one U(1) factor and has the Fayet-Iliopoulos (FI) D-term

$$
-\zeta(D)
$$

in its Lagrangian. The space of FI parameter $\zeta$ is decomposed into chambers called "phases" by the pattern of classical gauge symmetry breaking. Typically, the gauge group is broken to its finite subgroup when $\zeta$ is inside a phase, and a continuous unbroken subgroup shows up when $\zeta$ reaches its boundary. With $\mathcal{N}=2$ or more supersymmetry, the vector multiplet has at least one scalar component, and hence the appearance of continuous unbroken gauge symmetry means emergence of non-compact flat direction, called the Coulomb branch. In particular, we expect a phase transition as $\zeta$ moves from one phase to another through the mutual boundary. This is unlike in two dimensional models [2] where the actual transition is avoided by turning on the theta angle which can lift the Coulomb branch.

Witten index enjoys the usual good properties such as integrality and deformation invariance, under the condition that there is no flat direction. Suppose the only possible source of non-compactness is unbroken gauge symmetry. Then, the index enjoys the good property and does not depend on $\zeta$ as long as it is inside a phase in which the gauge symmetry is broken to a finite subgroup. However, as $\zeta$ approaches a phase boundary, the index ceases to have that property. If $\zeta$ enters another phase, it may regain that property but there is no reason for it to have the same value as in the original phase. Namely, Witten index may jump as $\zeta$ moves from one phase to another. This behaviour, called the "wall crossing", is a distinguished feature of one dimensional models. In two dimensions, wall crossing will not happen since the flat direction can be avoided by turning on the theta angle. Absence of wall crossing is also observed in three dimensional models [3]. 
The goal of the present paper is to compute the Witten index in one dimensional gauged linear sigma models, to see how it depends on the phase, and to understand the physics of the wall crossing.

Apart from its own interest, the present work has a strong motivation in string theory. An effective theory of D-particles in Type II superstring theory is provided by the $\mathcal{N}=16$ maximally supersymmetric SU( $n)$ matrix quantum mechanics [4], or by a class of gauged linear sigma models called quiver quantum mechanics [5-7], depending on the background. BPS bound states of D-particles correspond to supersymmetric ground states of the effective quantum mechanics, and the index computation in the latter is an extremely important problem. For example, the index for $\mathcal{N}=16$ theories [8, 9] is relevant for the M-theory conjecture. The spectrum of BPS states can change discontinuously as the theory is deformed. Such behavior, also called "wall-crossing," was initially discovered for BPS solitons in supersymmetric field theory ( $d=2$ BPS kinks [10], $d=4$ BPS solitons in strongly coupled [11-13] and weakly coupled [14-18] regimes) and then for BPS black holes in supergravity [19].

When the effective theory is given by a gauged linear sigma model, it is natural to expect that the two notions of wall crossing are related. After the pioneering works on Dbrane stability and the rôle of FI parameter [20,21], the relationship was first discussed in detail by F. Denef [22] when the effective theory is a simple class of $\mathcal{N}=4$ quiver quantum mechanics. The decay of BPS bound states, which had been known to be due to infinite separation of constituents $[15-17,19]$, was rephrased by Denef as the run away of the ground state wavefunctions to infinity in the Coulomb branch of the quiver theory [22, 23]. Around the same time, Reineke gave a master formula for counting the index in the geometric Higgs description [24], although this was limited to tree-like quiver theories.

Recently, there have been renewed efforts for systematic and explicit counting of index for all $\mathcal{N}=4$ quiver quantum mechanics [25-33], but much of these efforts rely on approximate schemes, either Coulomb or Higgs. The Coulomb approach in particular is physically attractive, as it explains the origin of wall-crossing behavior intuitively, and, for theories without superpotential, has been successfully demonstrated to be equivalent [34] to predictions via the Kontsevich-Soibelman algebra [35-37]. For theories with superpotentials, such an approximate scheme often fails dramatically and only a limited subset of such theories have been explored so far [23, 30-32, 38]. Nevertheless these new studies lead to a glimpse of very rich and surprising vacuum structure, whereby emerged the concept of quiver invariants and their proposed role as microstates of BPS black holes in $d=4 \mathcal{N}=2$ theories. In view of these new developments, the need for a more efficient and faithful method for computing the index of gauged quantum mechanics is all the more pressing.

The present paper offers a sweeping new approach to the index computation, which does not resort to any truncation or approximation to one sector, and thus is capable of computing full quantum mechanics index exactly, with wall-crossing taken into account. For quiver quantum mechanics, in particular, this opens up a new line of attack where both wall-crossing behavior and quiver invariants can be computed simultaneously.

To compute the index, we employ the method of supersymmetric localization, extending the recent computation of elliptic genus of two-dimensional $(0,2)$ gauge theories $[39,40]$ to quantum mechanics. In one dimension, the classical Coulomb branch cannot be lifted by 


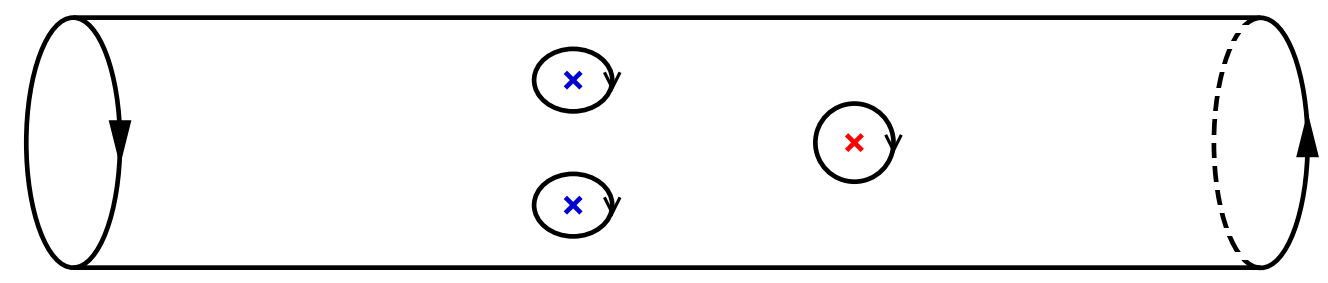

Figure 1. The moduli space $\mathfrak{M}$ for a $U(1)$ gauge theory.

quantum corrections, unlike in two dimensions where it can [41, 42]. This is one reason to limit our target to gauged linear sigma models with phases in which the gauge symmetry is classically broken to its finite subgroup. We may also turn on twist by a global symmetry that commutes with an $\mathcal{N}=2$ supersymmetry. This is necessary especially when the theory has a non-compact Higgs branch. If the global symmetry has a compact set of fixed points, the twist or the corresponding mass term lifts the flat direction, and the twisted index regains the good property. The index to compute is

$$
I\left(\mathrm{e}^{2 \pi i \mathbf{G}^{F}(z)}\right)=\operatorname{Tr}_{\mathcal{H}}(-1)^{F} \mathrm{e}^{2 \pi i \mathbf{G}^{F}(z)} \mathrm{e}^{-\beta H},
$$

where $\mathrm{e}^{2 \pi i \mathbf{G}^{F}(z)}$ is the twist. Outline of computation is as follows.

The index is realized as the path integral on the circle, and the main idea of localization is to take the limit of vanishing gauge coupling constant $e \rightarrow 0$. The path integral first localizes on the supersymmetric configurations for the $\mathcal{N}=2$ vector multiplet, which consists of a pair of the holonomy on the circle and the constant scalar that commute with each other. The moduli space is $\mathfrak{M}=(T \times \mathfrak{t}) / W$, where $T$ is a maximal torus of the gauge group, $\mathfrak{t}$ is its Lie algebra and $W$ is the Weyl group. To be precise, we need to excise from $T \times \mathfrak{t}=: \tilde{\mathfrak{M}}$ a neighborhood $\Delta_{\varepsilon}$ of the union of singular hyperplanes in which the scalar components of the $\mathcal{N}=2$ chiral multiplets have zero modes. It turns out that the integrand is a total derivative, and by Stokes theorem we have an integral on the boundary of $\tilde{\mathfrak{M}} \backslash \Delta_{\varepsilon}$. The boundary consists of $-\partial \Delta_{\varepsilon}$ as well as the boundary at infinity $\partial \tilde{\mathfrak{M}}=T \times \partial \mathfrak{t}$, which exists because $\mathfrak{t}$ is non-compact. See figure 1. Presence of the latter boundary is the main new feature in the one-dimensional theories compared to the two-dimensional theories in which the moduli space was a compact space $(T \times T) / W$. And this presence of infinity is the main reason for the wall crossing. This is in accord with the picture of wall crossing that is associated with the emergence of the Coulomb branch, and this non-compact $\mathfrak{t}$ is indeed the Coulomb branch itself!

At each boundary component, we have an integral of the Cartan zero mode of the auxiliary D-field, which is the $\mathcal{N}=2$ superpartner of the coordinates of $\mathfrak{M}$. The integral is of the form

$$
\int \frac{\mathrm{d} Q(D)}{Q(D)} \exp \left(-\frac{1}{2 e^{2}} D^{2}-i \zeta(D)\right)
$$

where $Q$ is an element of $i \mathfrak{t}^{*}$ that depends on the component of $\partial\left(\tilde{\mathfrak{M}} \backslash \Delta_{\varepsilon}\right)$ under consideration. At the boundary $-\partial \Delta_{\varepsilon}$, the integral can be processed in exactly the same way as in $[39,40]$. There, we have chosen an arbitrary generic element $\eta \in i t^{*}$ that specifies 
the way to deform the $D$ integration contour. That procedure resulted in having a sum over the isolated intersections of singular hyperplanes, where the summand is the so called $J K$ residue [43-45] of a meromorphic form on $\tilde{\mathfrak{M}}$ that is obtained by the one-loop integral. There, the integral (1.3) is either zero or picks up the simple pole at $Q(D)=0$. In either way, the $\zeta$ dependence is washed away. The residues depends only on $\eta$. The main question is what to do with the component at infinity $\partial \tilde{\mathfrak{M}}$ in which we put $Q=Q_{\infty}$. There, we need to evaluate (1.3) directly, along a contour specified by $\eta$. The simplest way to proceed is to set

$$
\eta=\zeta, \quad Q_{\infty}=\zeta .
$$

Then, it is the integral over $\zeta(D)$ along the contour $\mathbf{R}-i \epsilon$.

At this stage we recall that we are taking the limit $e \rightarrow 0$, which is potentially singular. The limit is regular, however, if $\zeta$ is inside a phase where the gauge group is broken to a finite subgroup, and if the Higgs mass scale $M_{H}=e \sqrt{|\zeta|}$ is held non-zero. That is, at the same time as $e \rightarrow 0$, we scale up $\zeta$ so that $\zeta^{\prime}=e^{2} \zeta$ is held at a fixed value inside the phase. In this scaling limit, which may be called the Higgs scaling, the $\zeta(D)$ integral vanishes. To see this, we scale the $\zeta$ component of $D$ as $e^{2} D^{\prime}$. Then, (1.3) is proportional to

$$
\int_{\mathbf{R}-i \epsilon} \frac{\mathrm{d} \zeta^{\prime}\left(D^{\prime}\right)}{\zeta^{\prime}\left(D^{\prime}\right)} \exp \left(-\frac{e^{2}}{2} D^{\prime 2}-i \zeta^{\prime}\left(D^{\prime}\right)\right) \stackrel{e \rightarrow 0}{\longrightarrow} \int_{\mathbf{R}-i \epsilon} \frac{\mathrm{d} \zeta^{\prime}\left(D^{\prime}\right)}{\zeta^{\prime}\left(D^{\prime}\right)} \exp \left(-i \zeta^{\prime}\left(D^{\prime}\right)\right)=0 .
$$

To summarize, with the choice (1.4) and in the Higgs scaling, all the integrals at infinity vanish. We are therefore left with the sum over isolated intersections of singular hyperplanes,

$$
I\left(\mathrm{e}^{2 \pi i \mathbf{G}^{F}(z)}\right)=\frac{1}{|W|} \sum_{p} \underset{p}{\operatorname{JK}-\operatorname{Res}}(Q(p), \zeta)\left[g(u, z) \mathrm{d}^{\ell} u\right]
$$

where $Q(p)$ is the set of charges of the chiral multiplets that define the singular hyperplanes that meet at $p, g(u, z)$ is the result of one-loop integral under the supersymmetric background, and $\mathrm{d}^{\ell} u$ is a correctly normalized holomorphic volume form on $\tilde{\mathfrak{M}}$ ( $\ell$ is the rank of the gauge group).

What happens if $\zeta$ moves from one phase to another? If we keep $\eta$ and $Q_{\infty}$ at the initial value of $\zeta$ during the process, nothing happens to the sum over isolated intersections of the singular hyperplanes. On the other hand, the integrals at infinity may become non-zero, since $\operatorname{Im} \zeta(D)$ may go from negative to positive: the integral (1.5) does not vanish if the contour $\mathbf{R}-i \epsilon$ is replaced by $\mathbf{R}+i \epsilon$. Therefore, the change of the index can be expressed as a certain sum over integrals at infinity. This matches with the picture of wall crossing due to the emergence of the Coulomb branch. In fact, one can sum up the integrals at infinity and make the relation to the Coulomb branch more precise, at least for a "simple wall crossing". A phase boundary is called simple when the unbroken gauge group there is isomorphic to $\mathrm{U}(1)$. Any phase boundary is simple for a general theory with Abelian gauge groups, and many are simple also in non-Abelian theories. At such a simple phase boundary, the states that are responsible for the wall crossing can be analyzed reliably. They have wavefunctions supported on the mixed Coulomb-Higgs branch, and the analysis yields a wall crossing formula. And that formula matches precisely with the wall crossing formula obtained by summing up the integrals at infinity! 
The rest of the paper is organized as follows.

In section 2, we introduce the one-dimensional gauged linear sigma models with at least $\mathcal{N}=2$ supersymmetry and describe their symmetries. We define the indices we are going to study in this paper. To be precise, (1.2) is only a mnemonic, and the actual definition is only in (2.54) in general. We show that the index depends holomorphically on the twist parameter, thus motivating that mnemonic. We also prove that, in an effectively compact $\mathcal{N}=4$ theory, the ground states have charge zero under $\mathcal{N}=4$ flavor symmetries. We introduce the notion of phases, Coulomb, Higgs, mixed branches. We end with presenting three classes of examples illustrating the phase structures.

In section 3, we describe the effective theory on the Coulomb branch near the phase boundary of $\mathrm{U}(1)$ theories as well as the effective theory on the mixed branch near simple phase boundaries of a general theory. This analysis is new for $\mathcal{N}=2$ theories but is of course a review of [22] for $\mathcal{N}=4$ theories. A complete detail is presented for the $\mathcal{N}=2$ $\mathbf{C P}^{N-1}$ model. We present a wall crossing formula for the index, for the case of simple wall crossing, as a result of the Coulomb and mixed branch analysis.

Section 4 is the main section where we compute the Witten index, as outlined above.

In section 5 , we derive the wall crossing formula at simple phase boundary, first in $\mathrm{U}(1)$ theories, next in a general Abelian theory and then in a general non-Abelian theory. Complete agreement with Coulomb or mixed branch formula is observed.

The remaining sections are devoted to examples that illustrate our findings in the earlier sections. In section 6 , we compute the index for $\mathcal{N}=4$ theories, including the linear sigma models for Grassmannians and hypersurfaces in projective spaces, models with compact and non-compact Higgs branches with flavor symmetries, and "the two parameter model". We see that Coulomb and mixed branch analysis can tell us also about Hodge diamond, which carries finer information than the index. We illustrate flavor decoupling in compact theories, and flavor non-decoupling in non-compact theories. In the non-compact theories, we find that the index without R-symmetry twist does not depend on the detail of the flavor twist even though its presence is certainly needed even to define the index. We also study the full Coulomb branch in "the two parameter model" and examine the meaning of the result.

Section 7 is devoted to $\mathcal{N}=4$ quiver theories which are important for $4 \mathrm{~d} \mathcal{N}=4$ BPS states counting and wall crossing phenomena. We start by illustrating the computation of the index for cyclic Abelian quivers and triangle non-Abelian quivers. The computation reproduces the known results on Abelian quivers, while it produces new results for nonAbelian quivers. We then move on to examine the idea of quiver invariants $[30,31]$ and an existing proposal [60] on how these enter the full indices of quiver theories as building blocks, with a brief introduction to that subject. Our new results provide a non-trivial test of this over-determined system.

In the final section 8 , we compute the index for $\mathcal{N}=2$ theories, including the linear sigma models for $\mathbf{C P}^{N-1}$ and Grassmannians, Distler-Kachru model, and triangle Abelian quivers. In $\mathbf{C P}^{N-1}$ and Grassmannian $G(k, N)$, we see that the result agrees with the Weyl character formula for representations of $\mathrm{SU}(N)$, in agreement with Borel-Weil-Bott theorem. In Distler-Kachru model, we observe an interesting process of wall crossing, 
where a pair of bosonic and fermionic states go down together to have zero energy before entering the wall crossing regime. We also illustrate the mixed branch wall crossing formula $\Delta I=I^{(\mathrm{H})} * \Delta_{C} I^{(\mathrm{C})}$.

In appendix we describe $1 \mathrm{~d} \mathcal{N}=2$ superfield formalism.

After this work was completed, presented [46, 47], and at the stage of being edited, two papers with partial overlaps [48, 49] appeared in the ArXiv.

\section{Notational convention:}

1. In the rest of this paper, we denote a compact Lie group by an upper case roman character and its Lie algebra by the lower case Gothic character, $G$ and $\mathfrak{g}$ for example. We denote their complexifications by putting subscript $\mathbf{C}$, as $G_{\mathbf{C}}$ and $\mathfrak{g}_{\mathbf{C}}$. We call elements of $\mathfrak{g} \subset \mathfrak{g}_{\mathbf{C}}$ pure imaginary, while elements of $i \mathfrak{g} \subset \mathfrak{g}_{\mathbf{C}}$ are called real. This is motivated by the fact that the Lie algebra of $\mathrm{U}(1)$ is naturally the vector space $i \mathbf{R}$ of pure imaginary numbers. For a given compact Lie group, say $G$, we shall write $T$ and $\mathfrak{t}$ for a maximal torus of $G$ and its Lie algebra, $N_{T}$ for the normalizer of $T$ in $G, W$ for the associated Weyl group $N_{T} / T, Z_{G}$ and $\mathfrak{z}$ for the center and its Lie algebra.

2. For a meromorphic function $f(z)$ of one variable, $\operatorname{res}_{z=a} f(z)$ is defined with a factor of extra $2 \pi i$ compared to the standard,

$$
\underset{z=a}{\operatorname{res}} f(z)=\oint_{a} \mathrm{~d} z f(z) \text {. }
$$

For example, $\operatorname{res}_{z=0}(1 / z)=2 \pi i$. The same applies to JK-Res as in (1.6). It is $(2 \pi i)^{\ell}$ times the standard one.

\section{Gauged linear sigma models in one dimension}

\subsection{The models}

We consider an $\mathcal{N}=2$ supersymmetric quantum mechanics with a compact gauge group $G$ and a certain set of matter multiplets. We first describe the variables, supersymmetry and Lagrangians. Much of the construction is obtained from the dimensional reduction of $2 d \mathcal{N}=(0,2)$ gauged linear sigma models [2], while a part is borrowed from the boundary interactions of $2 d \mathcal{N}=(2,2)$ theories [50]. We shall use the component expressions. See appendix A for the description in superspace.

\section{$\mathcal{N}=2$ supersymmetry}

An $\mathcal{N}=2$ supersymmetric quantum mechanics has the supercharges $\mathbf{Q}, \overline{\mathbf{Q}}$ and the Hamiltonian $H$ obeying

$$
\mathbf{Q}^{2}=\overline{\mathbf{Q}}^{2}=0, \quad\{\mathbf{Q}, \overline{\mathbf{Q}}\}=H .
$$

We may also have the R-charge $\boldsymbol{J}$ which obeys

$$
[\boldsymbol{J}, \mathbf{Q}]=-\mathbf{Q}, \quad[\boldsymbol{J}, \overline{\mathbf{Q}}]=\overline{\mathbf{Q}}, \quad[\boldsymbol{J}, H]=0
$$


$\mathbf{Q}$ and $\overline{\mathbf{Q}}$ are the adjoints of each other while $H$ and $\boldsymbol{J}$ are self adjoint. We shall write the supersymmetry transformation of field variables by

$$
\delta \mathcal{O}=[-i \epsilon \mathbf{Q}+i \bar{\epsilon} \overline{\mathbf{Q}}, \mathcal{O}]
$$

where $\epsilon$ and $\bar{\epsilon}$ are complex conjugate pair of fermionic variational parameters which have $\mathrm{R}$-charges +1 and -1 respectively.

\section{Vector multiplet}

The vector multiplet consists of a gauge field $v_{t}$, a scalar field $\sigma$, a complex conjugate pair of fermions $\lambda, \bar{\lambda}$, and an auxiliary field $D$, which are all valued in the adjoint representation of $G$. ( $v_{t}, \sigma$ and $D$ are "real" in the convention stated in the introduction.) They transform under the $\mathcal{N}=2$ supersymmetry as

$$
\begin{aligned}
\delta v_{t} & =-\delta \sigma=\frac{i}{2} \epsilon \bar{\lambda}+\frac{i}{2} \bar{\epsilon} \lambda, \\
\delta \lambda & =\epsilon\left(D_{t} \sigma+i D\right), \\
\delta D & =\frac{1}{2} \epsilon D_{t}^{(+)} \bar{\lambda}-\frac{1}{2} \bar{\epsilon} D_{t}^{(+)} \lambda .
\end{aligned}
$$

Here and in what follows, for a field $\mathcal{O}$ in a representation of $G$, we write $D_{t} \mathcal{O}:=\dot{\mathcal{O}}+i v_{t} \mathcal{O}$ for the covariant derivative and put

$$
D_{t}^{( \pm)} \mathcal{O}:=D_{t} \mathcal{O} \pm i \sigma \mathcal{O}
$$

Note that $\lambda$ is chiral, $\{\overline{\mathbf{Q}}, \lambda\}=0$. The supersymmetric kinetic term is

$$
L_{\text {gauge }}=\frac{1}{2 e^{2}} \operatorname{Tr}\left[\left(D_{t} \sigma\right)^{2}+i \bar{\lambda} D_{t}^{(+)} \lambda+D^{2}\right] .
$$

For an adjoint invariant linear form $\zeta: i \mathfrak{g} \rightarrow \mathbf{R}$, we have the Fayet-Iliopoulos term

$$
L_{\mathrm{FI}}=-\zeta(D) .
$$

Note that $\zeta$, which belongs to $i\left(\mathfrak{g}^{*}\right)^{G} \cong i\left(\mathfrak{t}^{*}\right)^{W}$, can be regarded as an element of $i \mathfrak{z}^{*}$ since $\left(\mathfrak{t}^{*}\right)^{W} \hookrightarrow \mathfrak{t}^{*} \rightarrow \mathfrak{z}^{*}$ is an isomorphism.

\section{Chiral multiplet}

A chiral multiplet consists of a scalar field $\phi$ and a fermion $\psi$ which are valued in a unitary representation $V_{\text {chiral }}$ of $G$. The supersymmetry transformations are

$$
\begin{aligned}
\delta \phi & =-\epsilon \psi \\
\delta \psi & =i \bar{\epsilon} D_{t}^{(+)} \phi .
\end{aligned}
$$

Note that $\phi$ is chiral, $[\overline{\mathbf{Q}}, \phi]=0$. The supersymmetric kinetic term is

$$
L_{\text {chiral }}=D_{t} \bar{\phi} D_{t} \phi+i \bar{\psi} D_{t}^{(-)} \psi+\bar{\phi}\left\{D-\sigma^{2}\right\} \phi-i \bar{\phi} \lambda \psi+i \bar{\psi} \bar{\lambda} \phi .
$$




\section{Fermi multiplet}

A fermi multiplet consists of a fermion $\eta$ and an auxiliary field $F$ which are valued in a unitary representation $V_{\text {fermi }}$ of $G$. The supersymmetry transformations are

$$
\begin{aligned}
\delta \eta & =\epsilon F+\bar{\epsilon} E(\phi), \\
\delta F & =\bar{\epsilon}\left(-i D_{t}^{(+)} \eta+\psi^{i} \partial_{i} E(\phi)\right),
\end{aligned}
$$

where $(\phi, \psi)$ is a chiral multiplet in a representation $V_{\text {chiral }}$ of $G$ and $E: V_{\text {chiral }} \rightarrow V_{\text {fermi }}$

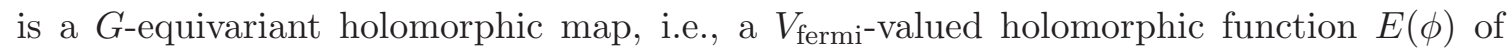
$\phi$ satisfying $E(g \phi)=g E(\phi)$. Note that $\{\overline{\mathbf{Q}}, \eta\}=-i E(\phi)$. Thus, $\eta$ is chiral only when $E(\phi)$ is trivial, in which case the multiplet may be called a chiral fermi multiplet. The supersymmetric kinetic term is

$$
L_{\mathrm{fermi}}=i \bar{\eta} D_{t}^{(+)} \eta+\bar{F} F-\overline{E(\phi)} E(\phi)-\bar{\eta} \partial_{i} E(\phi) \psi^{i}-\bar{\psi}^{\bar{\imath}} \partial_{\bar{\imath}} \overline{E(\phi)} \eta .
$$

\section{Superpotential}

For a chiral multiplet $(\phi, \psi)$ in a representation $V_{\text {chiral }}$ and a fermi multiplet $(\eta, F)$ in another representation $V_{\text {fermi }}$ as above, let $J: V_{\text {chiral }} \rightarrow V_{\text {fermi }}^{*}$ be a $G$-equivariant holomorphic map, such that $J(\phi) E(\phi)=0$. Then, $J(\phi) \eta$ is chiral, $\{\overline{\mathbf{Q}}, J(\phi) \eta\}=0$. In such a case, we have a supersymmetric interaction terms,

$$
L_{J}=\psi^{i} \partial_{i} J(\phi) \eta-J(\phi) F+\text { c.c.. }
$$

We shall call this the F-term associated to the superpotential $\mathfrak{W}=J(\phi) \eta$.

\section{Global anomaly}

As always in gauge theory, we need to impose the Gauss law constraint. Here we would like to point out that a non-trivial condition is required for that to be possible.

Let us consider a (not necessarily supersymmetric) gauge theory with a gauge group $G$, a bosonic variable in some representation of $G$ and a fermion pair, $\eta$ and $\bar{\eta}$, which take values in a unitary representation $V_{F}$ of $G$ and its dual $V_{F}^{*}$ respectively. We assume Lagrangian of the form

$$
L=i \bar{\eta} D_{t} \eta+\cdots=i \sum_{\alpha, \beta=1}^{d_{F}} \bar{\eta}_{\alpha}\left(\delta_{\beta}^{\alpha} \frac{\mathrm{d}}{\mathrm{d} t}+i\left(v_{t}\right)_{\beta}^{\alpha}\right) \eta^{\beta}+\cdots,
$$

where $d_{F}=\operatorname{dim} V_{F}$ and $+\cdots$ are terms that do not involve the time derivatives of $\eta$ and $\bar{\eta}$. Quantization of the fermionic variables is standard. The canonical anticommutation relation is $\left\{\eta^{\alpha}, \bar{\eta}_{\beta}\right\}=\delta_{\beta}^{\alpha},\left\{\eta^{\alpha}, \eta^{\beta}\right\}=\left\{\bar{\eta}_{\alpha}, \bar{\eta}_{\beta}\right\}=0$, and the hermiticity is $\eta^{\alpha \dagger}=\bar{\eta}_{\alpha}$. The space of states $\mathcal{H}_{F}$ is built on a "vacuum" $|0\rangle$ annihilated by all $\eta^{\alpha}$ 's,

$$
|0\rangle, \quad \bar{\eta}_{\alpha}|0\rangle, \quad \bar{\eta}_{\alpha} \bar{\eta}_{\beta}|0\rangle, \quad \ldots, \quad \bar{\eta}_{1} \cdots \bar{\eta}_{d_{F}}|0\rangle .
$$

Gauss law requires physical states to be $G$-invariant. For this, we need to specify how $G$ acts on the states. Throughout the paper, we take the standard quantization rule where $\eta$ 
and $\bar{\eta}$ play symmetric rôles. At the infinitesimal level, this yields the following expression for the gauge charge corresponding to $\xi \in \mathfrak{g}$

$$
\mathbf{G}(\xi)=\frac{1}{2}[\bar{\eta}, \xi \eta]+\cdots=\frac{1}{2} \xi_{\beta}^{\alpha}\left[\bar{\eta}_{\alpha}, \eta^{\beta}\right]+\cdots,
$$

where $+\cdots$ is the bosonic contribution which is unambiguous. For example, $\mathbf{G}(\xi)$ acts on the "vacuum" $|0\rangle$ by multiplication of $-\frac{1}{2} \operatorname{tr}_{V_{F}}(\xi)$. The question is whether this $\mathfrak{g}$-action lifts to a $G$-action. The condition is that $g \in G \mapsto \operatorname{det}_{V_{F}}^{\frac{1}{2}} g \in \mathrm{U}(1)$ is a well-defined group homomorphism. That is, the one-dimensional representation $\operatorname{det} V_{F}=\wedge^{d_{F}} V_{F}$ must have a square root. Absence of a square root can be regarded as a global anomaly. Suppose there is one, $\operatorname{det}^{\frac{1}{2}} V_{F}$. (If $G$ is connected, it is unique when it exists. If there is an ambiguity, we make a choice.) Then, as the space of (fermionic) states we can take

$$
\mathcal{H}_{F} \cong \operatorname{det}^{-\frac{1}{2}} V_{F} \otimes \wedge V_{F} .
$$

This global anomaly is in fact the same as the more familiar form of global anomaly, which is usually discussed in the path-integral quantization. For illustration, let us take the system of a single $\eta, \bar{\eta}$ pair where $\eta$ has charge one under $G=\mathrm{U}(1)$, with the simple Lagrangian $L=i \bar{\eta} D_{t} \eta$, and consider the partition function on the Euclidean circle of circumference $\beta$ with anti-periodic boundary condition. Assuming that $v_{\tau}$ is a constant $-2 \pi a / \beta$, the Dirac operator is diagonalized by the Fourier modes $\mathrm{e}^{-2 \pi i \mathrm{~m} \tau / \beta}$, and the partition function is the product of the eigenvalues

$$
Z_{S^{1}}(a)=C \prod_{\mathrm{m} \in \mathbf{Z}+\frac{1}{2}}\left(\frac{2 \pi m}{\beta}+\frac{2 \pi a}{\beta}\right) .
$$

where $C$ is a normalization constant. $a$ and its integer shifts are all gauge equivalent since they are related by large gauge transformations. Indeed the set of eigenvalues is invariant under the shifts. However, the partition function is not: as we continuously deform $a$ to $a+1,(2.17)$ changes by a sign, since a single eigenvalue of the Dirac operator goes from negative to positive. This is the standard statement of global anomaly. To relate this to the above discussion, we note that $Z_{S^{1}}(a)$ is identified as

$$
\operatorname{Tr}_{\mathcal{H}_{F}} \mathrm{e}^{2 \pi i \mathbf{G}(a)},
$$

and also that $(2.17)$ can be computed as

$$
C^{\prime} \prod_{\mathrm{m}=\frac{1}{2}}^{\infty}\left(1-\left(\frac{a}{\mathrm{~m}}\right)^{2}\right)=\frac{C^{\prime}}{2}\left(\mathrm{e}^{\pi i a}+\mathrm{e}^{-\pi i a}\right),
$$

for another normalization constant $C^{\prime}$. We see that the symmetric quantization (2.15) agrees with this result, and that the globaly anomaly in the canonical quantization is really the same things as the globaly anomaly in the path-integral.

Let us apply this to our $\mathcal{N}=2$ supersymmetric system with gauge group $G$, a chiral multiplet and a fermi multiplet with values in $V_{\text {chiral }}$ and $V_{\text {fermi }}$. The fermion in this theory takes values in $V_{F}=\mathfrak{g}_{\mathbf{C}} \oplus V_{\text {chiral }} \oplus V_{\text {fermi }}$. Note that $\operatorname{det} \mathfrak{g}_{\mathbf{C}}$ has a square root. Thus, the anomaly free condition is

$$
\operatorname{det}\left(V_{\text {chiral }} \oplus V_{\text {fermi }}\right) \quad \text { has a square root. }
$$




\section{Wilson line}

An important class of supersymmetric interactions is provided by a supersymmetric version of the Wilson line. Let $M$ be a $\mathbf{Z}_{2}$ graded vector space with a hermitian inner product, on which the gauge group elements act as unitary operators, $\rho: G \rightarrow \mathrm{U}(M)$. We suppose that there is a $G$-equivariant holomorphic map $Q: V_{\text {chiral }} \rightarrow \operatorname{End}^{\text {od }}(M)$, whose values square to zero,

$$
\begin{aligned}
Q(g \phi) & =\rho(g) Q(\phi) \rho(g)^{-1}, \\
\partial_{\bar{\imath}} Q(\phi) & =0 \\
Q(\phi)^{2} & =0 .
\end{aligned}
$$

Let us put

$$
\mathcal{A}_{t}=\rho\left(v_{t}+\sigma\right)-\psi^{i} \partial_{i} Q(\phi)+\bar{\psi}^{\overline{ }} \partial_{\bar{\imath}} Q(\phi)^{\dagger}+\left\{Q(\phi), Q(\phi)^{\dagger}\right\} .
$$

Then, under the supersymmetry transformations (2.4) and (2.8), letting the variational parameters $\epsilon, \bar{\epsilon}$ depend on $t$, it transforms as

$$
\delta \mathcal{A}_{t}=\mathscr{D}_{t}\left(-i \bar{\epsilon} Q-i \epsilon Q^{\dagger}\right)+i \dot{\bar{\epsilon}} Q+i \dot{\epsilon} Q^{\dagger}
$$

with $\mathscr{D}_{t} Y:=\frac{\mathrm{d}}{\mathrm{d} t} Y+i\left[\mathcal{A}_{t}, Y\right]$. This means that matrix factor

$$
\operatorname{Pexp}\left(-i \int \mathcal{A}_{t} \mathrm{~d} t\right)
$$

has $\mathcal{N}=2$ supersymmetry and can be placed as a path-integral weight. Moreover, the $\dot{\epsilon}$ terms in (2.23) shows that the supercharges act on the space $M$ as

$$
\left.\overline{\mathbf{Q}}\right|_{M}=-i Q(\phi),\left.\quad \mathbf{Q}\right|_{M}=i Q(\phi)^{\dagger} .
$$

When the anomaly free condition (2.20) is met, $\rho: G \rightarrow \mathrm{U}(M)$ must be a genuine representation. One advantage of the Wilson line is that, even if the condition (2.20) is violated, the anomaly may be cancelled by choosing $\rho$ which fails to be a genuine representation of $G$ - we only need $\mathcal{H}_{F} \otimes(M, \rho)$ to be a well-defined representation.

A class of Wilson lines may be induced from a fermi multiplet. If we quantize the fermi multiplet, we obtain the space of states

$$
M=\operatorname{det}^{-\frac{1}{2}} V_{\text {fermi }} \otimes \wedge V_{\text {fermi }} .
$$

This factor itself may or may not be a genuine representation of $G$, but that does not matter as long as the total system is anomaly free. The interaction terms in (2.11) and (2.12) are nothing but $-\mathcal{A}_{t}$ with

$$
Q(\phi)=\bar{\eta} E(\phi)+J(\phi) \eta=E(\phi) \wedge+J(\phi)\lrcorner .
$$

Note that $Q(\phi)^{2}=0$ is equivalent to the condition $J(\phi) E(\phi)=0$. Note also that this expression for $Q(\phi)$ is consistent with the supersymmetry transformation - compare (2.10) and (2.25). This construction, however, is special in that the dimension of $M$ is a power of 2 . 


\section{$\mathcal{N}=4$ supersymmetric systems}

An important class of systems have $\mathcal{N}=4$ supersymmetry - in addition to $\mathbf{Q}=\mathbf{Q}_{+}$and $\overline{\mathbf{Q}}=\overline{\mathbf{Q}}_{+}$there is another set of supercharges $\mathbf{Q}_{-}$and $\overline{\mathbf{Q}}_{-}$which obey the same algebra as (2.1) and anticommute with $\mathbf{Q}_{+}$and $\overline{\mathbf{Q}}_{+}$.

The $\mathcal{N}=4$ vector multiplet consists of the $\mathcal{N}=2$ vector multiplet $\left(v_{t}, x_{3}, \lambda_{-}, D\right)$ and an $\mathcal{N}=2$ chiral multiplet $\left(\sigma, i \bar{\lambda}_{+}\right)$in the complexified adjoint representation. ${ }^{1}$ An $\mathcal{N}=4$ chiral multiplet consists of an $\mathcal{N}=2$ chiral multiplet $\left(\phi, \psi_{+}\right)$and an $\mathcal{N}=2$ fermi multiplet $\left(\psi_{-}, F\right)$ in the same representation, say $V$, with $E(\sigma, \phi)=\sigma \phi$. The $\mathcal{N}=4$ invariant kinetic terms are just the sum of the $\mathcal{N}=2$ invariant kinetic terms:

$$
\begin{aligned}
& \boldsymbol{L}_{\text {gauge }}=L_{\text {gauge }}\left(v_{t}, x_{3}, \lambda_{-}, D\right)+\frac{1}{2 e^{2}} L_{\text {chiral }}\left(\sigma, i \bar{\lambda}_{+}, *\right), \\
& \boldsymbol{L}_{\text {chiral }}=L_{\text {chiral }}\left(\phi, \psi_{+}, *\right)+L_{\text {fermi }}\left(\psi_{-}, F, *\right),
\end{aligned}
$$

where "*" stands for the $\mathcal{N}=2$ vector multiplet fields or $E=\sigma \phi$ with its superpartner. For a $G$-invariant holomorphic function $W(\phi)$ of $\phi \in V$, an $\mathcal{N}=4$ invariant superpotential term is obtained from the $\mathcal{N}=2 \mathrm{~F}$-term with the superpotential $\mathfrak{W}=-\partial_{i} W(\phi) \psi_{-}^{i}$ (i.e. set $J(\phi)=-\mathrm{d} W(\phi))$. The FI-term (2.7) is $\mathcal{N}=4$ supersymmetric by itself. Note that $J E=0$ follows from the $G$-invariance of $W$ and the anomaly free condition (2.20) is automatically satisfied.

$\mathcal{N}=4$ invariant systems are obtained from $4 d \mathcal{N}=1$ or $2 d \mathcal{N}=(2,2)$ systems by dimensional reduction. To write the Lagrangians in a more familiar form, let us introduce a triplet scalar $\mathbf{x}=\left(x_{1}, x_{2}, x_{3}\right)$ for $\sigma=x_{1}-i x_{2}$, and doublet fermions

$$
\overline{\boldsymbol{\lambda}}=\left(\bar{\lambda}_{-}, \bar{\lambda}_{+}\right), \quad \boldsymbol{\lambda}=\left(\begin{array}{c}
\lambda_{-} \\
\lambda_{+}
\end{array}\right), \quad \overline{\boldsymbol{\psi}}=\left(\bar{\psi}_{-}, \bar{\psi}_{+}\right), \quad \boldsymbol{\psi}=\left(\begin{array}{c}
\psi_{-} \\
\psi_{+}
\end{array}\right) .
$$

We also write

$$
D+\frac{1}{2}[\sigma, \bar{\sigma}]=\boldsymbol{D}
$$

Then, the $\mathcal{N}=4$ invariant Lagrangians can be written as

$$
\begin{aligned}
\boldsymbol{L}_{\text {gauge }} & =\frac{1}{2 e^{2}} \operatorname{Tr}\left[\left(D_{t} \mathbf{x}\right)^{2}+i \overline{\boldsymbol{\lambda}} D_{t} \boldsymbol{\lambda}+\boldsymbol{D}^{2}+\sum_{i<j}\left[x_{i}, x_{j}\right]^{2}-\overline{\boldsymbol{\lambda}}[\mathbf{x}, \boldsymbol{\lambda}]\right] \\
\boldsymbol{L}_{\text {chiral }} & =D_{t} \bar{\phi} D_{t} \phi+i \overline{\boldsymbol{\psi}} D_{t} \boldsymbol{\psi}+\bar{F} F+\bar{\phi}\left\{\boldsymbol{D}-\mathbf{x}^{2}\right\} \phi-\overline{\boldsymbol{\psi}} \boldsymbol{x} \boldsymbol{\psi}+i \bar{\phi} \boldsymbol{\lambda} \boldsymbol{\psi}-i \overline{\boldsymbol{\psi} \boldsymbol{\lambda}} \phi . \\
\boldsymbol{L}_{W} & =F^{i} \partial_{i} W(\phi)-\frac{1}{2} \boldsymbol{\psi}^{i} \boldsymbol{\psi}^{j} \partial_{i} \partial_{j} W(\phi)+\text { c.c. }, \\
\boldsymbol{L}_{\mathrm{FI}} & =-\zeta(\boldsymbol{D}) .
\end{aligned}
$$

Here we used $\not \mathbf{x}:=\sum_{j} \sigma_{j} x_{j}$ where $\sigma_{1}, \sigma_{2}, \sigma_{3}$ are the Pauli matrices. We also used the contractions $\boldsymbol{\lambda} \psi:=\lambda_{+} \psi_{-}-\lambda_{-} \psi_{+}$and $\overline{\boldsymbol{\psi} \boldsymbol{\lambda}}:=-\bar{\psi}_{+} \bar{\lambda}_{-}+\bar{\psi}_{-} \bar{\lambda}_{+}$, etc, as in [51]. The $\mathcal{N}=4$ supersymmetry transformation

$$
\delta \mathcal{O}=\left[i \epsilon_{+} \mathbf{Q}_{-}-i \epsilon_{-} \mathbf{Q}_{+}-i \bar{\epsilon}_{+} \overline{\mathbf{Q}}_{-}+i \bar{\epsilon}_{-} \overline{\mathbf{Q}}_{+}, \mathcal{O}\right]
$$

\footnotetext{
${ }^{1}$ We shall use a special notation for $\mathcal{N}=4$ theories: $(\sigma, \lambda)$ of the $\mathcal{N}=2$ vector part of the $\mathcal{N}=4$ vector multiplet is denoted by $\left(\mathrm{X}_{3}, \lambda_{-}\right)$and instead , " $\sigma$ " is used for the scalar component of the $\mathcal{N}=2$ chiral part of the $\mathcal{N}=4$ vector multiplet.
} 
is given by

$$
\begin{aligned}
\boldsymbol{\delta} v_{m} & =\frac{i}{2} \overline{\boldsymbol{\epsilon}}_{m} \boldsymbol{\lambda}-\frac{i}{2} \overline{\boldsymbol{\lambda}} \bar{\sigma}_{m} \boldsymbol{\epsilon}, \\
\boldsymbol{\delta} \boldsymbol{\lambda} & =\left(i \boldsymbol{D}+\sigma^{m n} v_{m n}\right) \boldsymbol{\epsilon}, \\
\boldsymbol{\delta} \boldsymbol{D} & =\frac{1}{2} \overline{\boldsymbol{\epsilon}}^{m} D_{m} \boldsymbol{\lambda}+\frac{1}{2} D_{m} \overline{\boldsymbol{\lambda}} \bar{\sigma}^{m} \boldsymbol{\epsilon}, \\
\boldsymbol{\delta} \phi & =\boldsymbol{\epsilon} \boldsymbol{\psi} \\
\boldsymbol{\delta} \boldsymbol{\psi} & =i \sigma^{m} \overline{\boldsymbol{\epsilon}} D_{m} \phi+\boldsymbol{\epsilon} F, \\
\boldsymbol{\delta} F & =i \overline{\boldsymbol{\epsilon}} \bar{\sigma}^{m} D_{m} \boldsymbol{\psi}-i \overline{\boldsymbol{\epsilon}} \overline{\boldsymbol{\lambda}} \phi .
\end{aligned}
$$

Here we follow the Wess-Bagger convention [51] for the contraction of spinors and the sigma matrices. $m, n$ run over $0,1,2,3$, and we write $v_{0}=v_{t}, v_{j}=x_{j}, v_{0 j}=D_{t} x_{j}, v_{j k}=i\left[x_{j}, x_{k}\right]$, $D_{0} \mathcal{O}=D_{t} \mathcal{O}, D_{j} \mathcal{O}=i x_{j} \mathcal{O}$. The $\mathcal{N}=2$ supersymmetry is obtained by setting $\epsilon_{+}=0$, $\bar{\epsilon}_{+}=0, \epsilon_{-}=\epsilon$, and $\bar{\epsilon}_{-}=\bar{\epsilon}$.

There is a no go theorem for Wilson line (2.22) in an $\mathcal{N}=4$ supersymmetric theory. In the present case, a Wilson line would be of the from

$$
\mathcal{A}_{t}=\rho\left(v_{t}+x_{3}\right)-\psi_{+}^{i} \partial_{i} Q+i \bar{\lambda}_{+}^{a} \partial_{\sigma^{a}} Q+\bar{\psi}_{+}^{\overline{ }} \partial_{\bar{\imath}} Q^{\dagger}-i \lambda_{+}^{a} \partial_{\bar{\sigma}^{a}} Q^{\dagger}+\left\{Q, Q^{\dagger}\right\} .
$$

The fields involved transform under the second $\mathcal{N}=2$ supersymmetry as

$$
\begin{aligned}
& \delta^{\prime \prime}\left(v_{t}+\mathrm{x}_{3}\right)=i\left(\epsilon_{+} \bar{\lambda}_{+}+\bar{\epsilon}_{+} \lambda_{+}\right), \quad \delta^{\prime \prime} \phi=\epsilon_{+} \psi_{-}, \quad \delta^{\prime \prime} \sigma=-i \bar{\epsilon}_{+} \lambda_{-}, \\
& \delta^{\prime \prime} \psi_{+}=-\bar{\epsilon}_{+} \bar{\sigma} \phi+\epsilon_{+} F, \quad \delta^{\prime \prime} \bar{\lambda}_{+}=-i \bar{\epsilon}_{+}\left\{\left(\boldsymbol{D}-i D_{t} \mathrm{x}_{3}\right)+\frac{1}{2}[\sigma, \bar{\sigma}]\right\} .
\end{aligned}
$$

It is clear that $\delta^{\prime \prime} \mathcal{A}_{t}$ cannot be written as $\mathcal{D}_{t} Y=\partial_{t} Y+i\left[\mathcal{A}_{t}, Y\right]$ for any $Y$. Therefore, the Wilson line does not preserve the $\mathcal{N}=4$ supersymmetry. Of course as remarked earlier, we do have a Wilson line if we quantize the $\mathcal{N}=2$ fermi multiplet, which is $\left(\psi_{-}, F\right)$ in the present case. If we do so, $-\mathcal{A}_{t}$ constitutes a part of the sum of (2.33) and (2.34), and is $\mathcal{N}=4$ supersymmetric only with the remaining part of the sum. The theorem states that there is no Wilson line which is $\mathcal{N}=4$ supersymmetric by itself.

\section{Global symmetry and real mass}

We recall that the R-symmetry group of $\mathcal{N}=2$ supersymmetry is $\mathrm{U}(1)$ generated by $\boldsymbol{J}$ under which the variational parameters $\epsilon$ and $\bar{\epsilon}$ have charges 1 and -1 . The R-charges of the component fields in the vector, chiral and fermi multiplets are as follows (the real variables $v_{t}, \sigma$ and $D$ cannot have any $\mathrm{R}$-charge):

$$
\begin{array}{|l|l|lc|lc|}
\hline & \lambda & \phi & \psi & \eta & F \\
\hline \boldsymbol{J} & 1 & r_{\mathrm{c}} & r_{\mathrm{c}}-1 & r_{\mathrm{f}} & r_{\mathrm{f}}-1 \\
\hline
\end{array}
$$

$r_{\mathrm{c}}: V_{\text {chiral }} \rightarrow V_{\text {chiral }}$ and $r_{\mathrm{f}}: V_{\text {fermi }} \rightarrow V_{\text {fermi }}$ are the R-charges of the chiral and the fermi multiplets. The system has U(1) R-symmetry when we can find $r_{\mathrm{c}}, r_{\mathrm{f}}$ and/or $r_{M}: M \rightarrow M$ 
so that $E(\phi), J(\phi)$ and/or $Q(\phi)$ have R-charges $r_{\mathrm{f}}+1,1-r_{\mathrm{f}}^{T}$, and/or 1 respectively in the sense that,

$$
\begin{aligned}
E\left(\omega^{r_{\mathrm{c}}} \phi\right) & =\omega^{r_{\mathrm{f}}+1} E(\phi), \\
J\left(\omega^{r_{\mathrm{c}}} \phi\right) & =J(\phi) \omega^{1-r_{\mathrm{f}}}, \\
\omega^{r_{M}} Q\left(\omega^{r_{\mathrm{c}}} \phi\right) \omega^{-r_{M}} & =\omega Q(\phi) .
\end{aligned}
$$

The system has a discrete R-symmetry when these hold for $\omega$ in a subset of $\mathrm{U}(1)$.

Suppose there is a set $h$ of unitary transformations $h_{\mathrm{c}}, h_{\mathrm{f}}$ and $h_{M}$ on $V_{\text {chiral }}, V_{\text {fermi }}$ and $M$ that commute with the gauge group action, obeying

$$
\begin{aligned}
E\left(h_{\mathrm{c}} \phi\right) & =h_{\mathrm{f}} E(\phi), \\
J\left(h_{\mathrm{c}} \phi\right) & =J(\phi) h_{\mathrm{f}}^{-1}, \\
Q\left(h_{\mathrm{c}} \phi\right) & =h_{M} Q(\phi) h_{M}^{-1} .
\end{aligned}
$$

Then, we have a global symmetry that acts on the chiral and fermi multiplets as $(\phi, \psi) \rightarrow$ $\left(h_{\mathrm{c}} \phi, h_{\mathrm{c}} \psi\right)$ and $(\eta, F) \rightarrow\left(h_{\mathrm{f}} \eta, h_{\mathrm{f}} F\right)$ while keeping the vector multiplet $\left(v_{t}, \sigma, \lambda, D\right)$ intact. It commutes with the supercharges $\mathbf{Q}$ and $\overline{\mathbf{Q}}$. We shall call it a flavor symmetry.

When there is a continuous group $G_{F}$ of flavor symmetries, the theory can be deformed by the following procedure: promote $G_{F}$ to a gauge group, turn on a supersymmetric configuration $\left(v_{t}^{F}, \sigma^{F}, D^{F}, \lambda^{F}\right)$ of its vector multiplet, and then demote it back to a flavor group by turning off the gauge coupling. In view of (2.4), the condition of supersymmetry is $D_{t}^{F} \sigma^{F}=0, D^{F}=0$ and $\lambda^{F}=0$. For example, we may turn on constant $v_{t}^{F}$ and $\sigma^{F}=m$ that commute with each other. This $m$ is an analog of real mass in $3 \mathrm{~d} \mathcal{N}=2$ theories and twisted mass in $2 d(2,2)$ theories, and shall be called real mass again. $v_{t}^{F}$ may be called flavor Wilson line. This deformation preserves the supersymmetry but deforms its algebra as

$$
\{\mathbf{Q}, \overline{\mathbf{Q}}\}=H-\mathbf{G}^{F}\left(v_{t}^{F}+m\right),
$$

where $\mathbf{G}^{F}(\xi)$ is the Noether charge of $\xi \in i \mathfrak{g}_{F}$. The parameters $v_{t}^{F}$ and $m$ enter into $L_{\text {chiral }}$, $L_{\text {fermi }}$ and $\mathcal{A}_{t}^{F}$. The latter two depends only on the combination $v_{t}^{F}+m$. $L_{\text {chiral }}$ depends also on $v_{t}^{F}-m$ but its variation is Q-exact: for $\xi \in i \mathfrak{g}_{F}$ we have

$$
\begin{aligned}
\left(\delta_{\xi}^{v_{t}^{F}}-\delta_{\xi}^{m}\right) L_{\text {chiral }} & =-i \bar{\phi} \xi D_{t}^{(+)} \phi+i D_{t}^{(+)} \bar{\phi} \xi \phi-2 \bar{\psi} \xi \psi \\
& =i[\mathbf{Q}, \bar{\psi} \xi \phi]-i[\overline{\mathbf{Q}}, \bar{\phi} \xi \psi] .
\end{aligned}
$$

This means that supersymmetric correlators depend on $v_{t}^{F}$ and $m$ only through the combination $v_{t}^{F}+m$.

The R-symmetry group of $\mathcal{N}=4$ supersymmetry is $O(4)$ which rotates the four real components of the supercharges $\mathbf{Q}_{ \pm}$and $\overline{\mathbf{Q}}_{ \pm}$. The R-charge $\boldsymbol{J}$ of the $\mathcal{N}=2$ subalgebra is $\boldsymbol{J}_{+}$that rotates the phases of $\mathbf{Q}=\mathbf{Q}_{+}$and $\overline{\mathbf{Q}}=\overline{\mathbf{Q}}_{+}$only. The subgroup of elements that commutes with $\mathbf{Q}_{+}$and $\overline{\mathbf{Q}}_{+}$is generated by the charge $\boldsymbol{J}_{-}$which rotates the phases of $\mathbf{Q}_{-}$ and $\overline{\mathbf{Q}}_{-}$only. In fact, $\boldsymbol{J}_{ \pm}$generate the maximal torus of $\mathrm{U}(2) \subset O(4)$. Its $\mathrm{SU}(2)$ subgroup is always a symmetry in the systems introduced in the previous section, under which $\mathbf{x}$ 
and $\boldsymbol{\lambda}$ form a triplet and a doublet. In this paper, we shall consider theories which are invariant also under the central $\mathrm{U}(1)$ subgroup of $\mathrm{U}(2)$. This requires the superpotential to be homogeneous, that is, there is an endomorphism $R: V \rightarrow V$ under which

$$
W\left(\omega^{R} \phi\right)=\omega^{2} W(\phi)
$$

Then, the system is invariant under $\mathrm{SU}(2) \times \mathrm{U}(1)$ with the following charge assignment, ${ }^{2}$

\begin{tabular}{|r|c|cc|cc|cc|}
\hline & $\lambda_{-}$ & $\sigma$ & $\bar{\lambda}_{+}$ & $\phi$ & $\psi_{+}$ & $\psi_{-}$ & $F$ \\
\hline $\boldsymbol{J}=\boldsymbol{J}_{+}$ & 1 & 1 & 0 & $\frac{R}{2}$ & $\frac{R}{2}-1$ & $\frac{R}{2}$ & $\frac{R}{2}-1$ \\
$\boldsymbol{J}_{-}$ & 0 & -1 & -1 & $\frac{R}{2}$ & $\frac{R}{2}$ & $\frac{R}{2}-1$ & $\frac{R}{2}-1$ \\
\hline
\end{tabular}

Since $\boldsymbol{J}_{-}$commutes with the $\mathcal{N}=2$ supercharges $\mathbf{Q}_{+}$and $\overline{\mathbf{Q}}_{+}$, it can be considered as a flavor symmetry of the $\mathcal{N}=2$ theory. Indeed, the $\boldsymbol{J}_{-}$charges in the table (2.50) are the same for the fields in each $\mathcal{N}=2$ multiplet, and zero for $\lambda_{-}$in the $\mathcal{N}=2$ vector multiplet. If there is a unitary transformation $h: V \rightarrow V$ that leaves $W(\phi)$ invariant, then we have a flavor symmetry that commutes with the $\mathcal{N}=4$ supersymmetry generators. For a continuous group of such flavor symmetries, we have a deformation of the theory by a supersymmetric background $v_{t}^{F}, \mathbf{x}^{F}=\left(m_{1}, m_{2}, m_{3}\right)$ of the flavor group. Note that the four elements $v_{t}^{F}, m_{1}, m_{2}, m_{3}$ must commute with each other. Extending the terminology in $2 \mathrm{~d}(2,2)$ theories, we may refer to $\widetilde{m}=m_{1}+i m_{2}$ as the twisted mass. This deformation preserves the supersymmetry and the supersymmetry algebra is deformed as

$$
\begin{aligned}
\left\{\mathbf{Q}_{ \pm}, \overline{\mathbf{Q}}_{ \pm}\right\} & =H-\mathbf{G}^{F}\left(v_{t}^{F} \pm m_{3}\right), \\
\left\{\mathbf{Q}_{-}, \overline{\mathbf{Q}}_{+}\right\} & =\mathbf{G}^{F}(\widetilde{m}), \quad\left\{\mathbf{Q}_{+}, \overline{\mathbf{Q}}_{-}\right\}=\mathbf{G}^{F}(\bar{m})
\end{aligned}
$$

When there is such a continuous flavor symmetry, there is an ambiguity in the choice of $R$. Unlike in theories in higher dimensions where we would like to find the "correct" R-charge that corresponds to the R-symmetry of the superconformal field theory in the infra-red limit, there is no distinguished choice in quantum mechanics.

\subsection{The index}

The main purpose of the present paper is to compute the Witten index [1] of $\mathcal{N}=2$ supersymmetric gauge theories, possibly with a twist by a flavor symmetry,

$$
I(h)=\operatorname{Tr}_{\mathcal{H}}\left((-1)^{F} \widehat{h} \mathrm{e}^{-\beta H}\right) .
$$

This can be identified as the path-integral on the Euclidean circle of circumference $\beta$ with the boundary condition $\mathcal{O}(\tau)=h \mathcal{O}(\tau+\beta)$ on all fields. This is equivalent to the circle with periodic boundary condition, but under the influence of the background flavor gauge field with holonomy $h$. Suppose there is a continuous group $G_{F}$ of flavor symmetries, and let us consider the index with a twist by an element of the form $h=\mathrm{e}^{i \xi}$ for $\xi \in i \mathfrak{g}_{F}$. Then, the

\footnotetext{
${ }^{2}$ The $J_{3}$ component of SU(2) and the U(1) generator $\boldsymbol{R}$ is related to $\boldsymbol{J}_{ \pm}$as $2 J_{3}=-\boldsymbol{J}_{-}+\boldsymbol{J}_{+}, \boldsymbol{R}=\boldsymbol{J}_{-}+\boldsymbol{J}_{+}$. $2 J_{3}$ and $\boldsymbol{R}$ are respectively the axial and vector R-charges in 2 d $(2,2)$ supersymmetry.
} 
flavor gauge field can be taken to be of the form $v_{\tau}^{F}=-\xi / \beta$. At the same time, we may also turn on the real mass $m \in i \mathfrak{g}_{F}$ that commutes with $\xi$. Then, we have a two parameter family of Witten index

$$
I(\xi, m)=\operatorname{Tr}_{\mathcal{H}_{m}}\left((-1)^{F} \mathrm{e}^{i \mathbf{G}_{m}^{F}(\xi)} \mathrm{e}^{-\beta H_{m}}\right),
$$

where $\left(\mathcal{H}_{m}, H_{m}, \mathbf{G}_{m}^{F}\right)$ is for the theory deformed by the real mass $m$. Due to the remark given in the previous subsection, it depends on $-i \xi / \beta+m$ but not on $-i \xi / \beta-m$. (Note that Wick rotation $t \rightarrow-i \tau$ does $v_{t}^{F} \rightarrow i v_{\tau}^{F}$.) That is, it is a holomorphic function of $\xi+i \beta m$.

The index enjoys the well known nice properties [1] when the theory is effectively compact, that is, when the spectrum is discrete and each level consists of finite number of square normalizable states. In particular, it receives contribution only from the finite number of supersymmetric ground states, $I(h)=\operatorname{Str}_{\mathcal{H}}$ SUSY $(\widehat{h})$. For the twist by an element $\mathrm{e}^{i \xi}$ of a continuous flavor group $G_{F}$, the function $\mathrm{e}^{i \xi} \mapsto \operatorname{Str}_{\mathcal{H}_{\text {SUSY }}}\left(\mathrm{e}^{i \mathbf{G}^{F}(\xi)}\right)$, being a (graded) character of a finite dimensional representation, has a holomorphic extension $\mathrm{e}^{i(\xi+i \beta m)} \mapsto \operatorname{Str}_{\mathcal{H}_{\text {SUSY }}}\left(\mathrm{e}^{i \mathbf{G}^{F}(\xi+i \beta m)}\right)$. By the uniqueness of holomorphic extension, it must agree with (2.54). That is,

$$
I(\xi, m)=\operatorname{Tr}_{\mathcal{H}}\left((-1)^{F} \mathrm{e}^{i \mathbf{G}^{F}(\xi+i \beta m)} \mathrm{e}^{-\beta H}\right) .
$$

This can also be shown directly: using the deformed supersymmetry algebra (2.47), we have $\mathrm{e}^{i \mathbf{G}_{m}^{F}(\xi)} \mathrm{e}^{-\beta H_{m}}=\mathrm{e}^{i \mathbf{G}_{m}^{F}(\xi+i \beta m)} \mathrm{e}^{-\beta\{\mathbf{Q}, \overline{\mathbf{Q}}\}}$, while the charge of a normalizable state is quantized and is constant under deformation. In either way, we have seen that the real mass plays a minor rôle for the index - it simply complexifies the twist parameter. In other words, the index with a complex twist parameter can be identified as the index with a unitary twist, of the theory deformed by a real mass.

Things are subtle when the theory is not effectively compact, which happens when the scalar potential has a non-compact flat direction. In such a theory, the index may not even be defined - the path-integral may diverge by the non-compact integral without exponential suppression; also, it is a delicate problem to define the trace of an operator on a non-separable Hilbert space. Here the flavor twist and/or the real mass $m$ play an essential rôle. When a maximal torus of the flavor group acts on the flat direction with a compact set of fixed points, then the twisted path-integral will converge, and that would provide a definition of the twisted index. On the other hand, with only such a twist, it is not clear if the operator definition as in the right hand side of (2.53) makes sense. If the real mass is turned on, however, the potential term $\bar{\phi} m^{2} \phi$ is introduced and the theory becomes effectively compact. Then, the index can certainly be defined in the operator formalism by (2.54). In what follows, to simplify the notation, we shall write the twisted index as (2.55) and refer to it as the index with complex flavor twist, even if the actual definition may only be (2.54).

One of our main interests in $\mathcal{N}=4$ theories is the index twisted by the R-symmetry $\boldsymbol{J}_{-}$that commutes with $\boldsymbol{J}=\boldsymbol{J}_{+}$,

$$
I\left(\mathbf{y}^{2 \boldsymbol{J}_{-}}\right)=\operatorname{Tr}_{\mathcal{H}}\left((-1)^{F} \mathbf{y}^{2 \boldsymbol{J}_{-}} \mathrm{e}^{-\beta H}\right)
$$


for $\mathbf{y} \in \mathbf{C}^{\times}$. Of course, if there is a flavor symmetry, i.e., $h: V \rightarrow V$ that leaves $W(\phi)$ invariant, we can also twist the index by that. For a continuous group of flavor symmetries, not only the twist but also the triplet mass deformation $\left(m_{1}, m_{2}, m_{3}\right)$ may be considered. However, the twisted mass deformation $\widetilde{m}=m_{1}+i m_{2}$ is forbidden in the presence of the R-twist $\mathbf{y}^{2 \boldsymbol{J}_{-}}$. This is because $\boldsymbol{J}_{-}$rotates the complex scalar $\sigma$ of the $\mathcal{N}=4$ vector multiplet, and hence would not be a symmetry if the twisted mass is turned on. Only the real mass $m_{3}$ is allowed and we have the complex flavor twist (along with $\mathbf{y}^{2 \boldsymbol{J}_{-}}$), as in the general $\mathcal{N}=2$ theories.

When the theory is effectively compact, the supersymmetric ground states have vanishing flavor charge. This can be shown as follows. Let us consider the twisted mass deformation. (This is just for the proof of the claim.) The deformed theory has a deformed supersymmetry algebra (2.52). Taking the $\widetilde{m}$ derivative and then setting $\widetilde{m}=0$, we find

$$
\left\{\mathbf{Q}_{-}, \delta_{\widetilde{m}} \overline{\mathbf{Q}}_{+}\right\}+\left\{\delta_{\widetilde{m}} \mathbf{Q}_{-}, \overline{\mathbf{Q}}_{+}\right\}=\mathbf{G}^{F}(\delta \widetilde{m}) .
$$

Suppose $|\Psi\rangle$ is a supersymmetric ground state. Applying the above operator identity to $|\Psi\rangle$, we see that $\mathbf{G}^{F}(\delta \widetilde{m})|\Psi\rangle$ is of the form $\mathbf{Q}_{-}|\alpha\rangle+\overline{\mathbf{Q}}_{+}|\beta\rangle$. Since $\mathbf{G}^{F}$ is a symmetry, $\mathbf{G}^{F}(\delta \widetilde{m})|\Psi\rangle$ is also a supersymmetric ground state. If $\Pi_{0}$ denotes the projection to the space of supersymmetric ground states, we find $\mathbf{G}^{F}(\delta \widetilde{m})|\Psi\rangle=\Pi_{0} \mathbf{G}^{F}(\delta \widetilde{m})|\Psi\rangle=\Pi_{0}\left(\mathbf{Q}_{-}|\alpha\rangle+\overline{\mathbf{Q}}_{+}|\beta\rangle\right)=0$. Since this holds for any $\delta \widetilde{m}$, this means that the ground state $|\Psi\rangle$ has vanishing flavor charge. Note that we needed the effective compactness of the theory so that the spectrum changes smoothly under the twisted mass deformation.

This means that the index does not depend on the twist by a continuous flavor symmetry. In particular, possible ambiguity in the R-charge assignment does not affect the index. Therefore, "the index with just the R-twist (2.56)" has an unambiguous meaning and that is our primary interest in effectively compact $\mathcal{N}=4$ theories.

When the theory is not effectively compact, the spectrum changes discontinuously when the twisted mass deformation is turned on, and the above argument does not apply. As remarked above in a general $\mathcal{N}=2$ theory, for a non-compact theory, flavor twist is very important even for having a well-defined index, and the result of course depends on the twist parameters.

The above proof does not apply to a theory with only $\mathcal{N}=2$ supersymmetry, even if it is compact. One might try to repeat the argument using the deformed algebra (2.47). But, as the real mass $m$ is deformed, the Hamiltonian also changes and one cannot conclude that the ground states have vanishing flavor charge. In fact, our primary interest in $\mathcal{N}=2$ theories is the dependence on the flavor twist. Also, presence of flavor twist is sometimes necessary for the localization computation.

\subsection{Phases}

Let us consider a system of $\mathcal{N}=2$ supersymmetric quantum mechanics with gauge group $G$, a chiral multiplet in $V_{\text {chiral }}$ and a fermi multiplet in $V_{\text {fermi }}$. As the Lagrangian, we take the sum of $L_{\text {gauge }}$ from (2.6), $L_{\text {chiral }}$ from (2.9), $L_{\text {fermi }}$ from (2.11), $L_{\mathrm{FI}}$ from (2.7) and $L_{J}$ from (2.12), and we may also include the Wilson line $-\mathcal{A}_{t}$ from (2.22). (Not all of them has to be non-trivial.) 
Our main interest is the space of supersymmetric ground states. Classically, supersymmetric vacua correspond to configurations of the scalar variables, $\sigma$ and $\phi$, at which the potential vanishes. The scalar potential of the system is

$$
\begin{aligned}
U(\sigma, \phi)= & |\sigma \phi|^{2}+\frac{e^{2}}{2}(\phi \bar{\phi}-\zeta)^{2}+|E(\phi)|^{2}+|J(\phi)|^{2} \\
& +\rho(\sigma)+\left\{Q(\phi), Q(\phi)^{\dagger}\right\} .
\end{aligned}
$$

The second and the fourth terms are obtained after integrating out the auxiliary fields $D$ and $F$ and called the $D$-term potential and the $F$-term potential respectively. $\frac{e^{2}}{2}(\cdot)^{2}$ is the quadratic form on $i \mathfrak{g}^{*}$ defined as the dual of $\frac{1}{2 e^{2}} \operatorname{Tr}(\cdot)^{2} . \phi \bar{\phi}$ inside that parenthesis is the moment map of the $G$ action on $V_{\text {chiral }}$ defined by $\langle\phi \bar{\phi}, \xi\rangle:=\bar{\phi} \xi \phi$. When $M$ has rank 2 or higher, the potential is valued in hermitian matrices. The term $\rho(\sigma)$ is not positive definite, which may appear strange in the presence of supersymmetry. However, as we will see, positivity does exist if the Gauss law is taken into account. Since that is a quantum effect, in the classical analysis which we present now, we shall simply forget about the term $\rho(\sigma)$. Then the vacuum equations are

$$
\begin{aligned}
\sigma \phi & =0, \\
\phi \bar{\phi} & =\zeta, \\
E(\phi)=0, \quad J(\phi) & =0, \quad\left\{Q(\phi), Q(\phi)^{\dagger}\right\}=0 .
\end{aligned}
$$

Notice that the FI parameter $\zeta$ enters into the second equation (2.60), called the Dterm equation. For a generic $\zeta$, this forces $\phi$ to have a non-zero value, which breaks the gauge group $G$ to its subgroup $G_{1}$ and forces $\sigma$ to lie in its Lie algebra. The space of solutions to the vacuum equations is in general a union of components labeled by the unbroken subgroup $G_{1}$. A component is called a Higgs branch if $G_{1}$ is a finite subgroup so that $\phi \neq 0$ and $\sigma=0$, a Coulomb branch if $G_{1}$ is $G$ itself so that $\phi=0$ and $\sigma \neq 0$, and a mixed branch if $G_{1}$ is a continuous proper subgroup of $G$ so that $\phi$ and $\sigma$ are both non-zero while obeying $\sigma \phi=0$. At each branch the gauge group is further broken to a subgroup of $G_{1}$ by the values of $\sigma$, generically to its maximal torus - thus the name "Coulomb". The pattern of gauge symmetry breaking depends very much on the value of $\zeta$. The space $i \mathfrak{z}^{*}$ of FI parameters is stratified by cones labelled by the symmetry breaking pattern. The cones of the maximal dimension shall be called the phases, following the terminology used in two dimensional systems. Cones of codimension one are called walls or phase boundaries. When the gauge group $G$ is Abelian, it is broken to a finite subgroup in each phase and to a rank one subgroup at each wall. When $G$ is non-Abelian, the situation can be different. Some models contain phases with continuous unbroken subgroups. Examples are the 1d versions of the $2 \mathrm{~d}$ models in $[41,42,52]$.

Inside a phase where the gauge group is broken to a finite subgroup and the vacuum manifold is a Higgs branch, we can often find an effective description of the system that correctly captures the supersymmetric ground states. Since the continuous part of the gauge group is completely broken, the vector multiplet together with the gauge orbit direction of the chiral multiplet acquire a non-zero mass by the Higgs mechanism. (In quantum 
mechanics, "mass" should better be rephrased as "frequency", but we shall keep the terminology used in higher dimensions.) By looking at the term $|\sigma \phi|^{2}$ in the potential and the D-term equation $\phi \bar{\phi}=\zeta$, we find that the mass is of the order of

$$
M_{H}=e \sqrt{|\zeta|}
$$

where $|\zeta|$ is the typical size of the FI parameter that is responsible for the gauge symmetry breaking. ${ }^{3}$ Therefore, the vector multiplet and the gauge orbit modes decouple from the physics at the energy scale $E$ below $M_{H}$.

When the FI parameter $\zeta$ is on a phase boundary, the space of classical vacua has mixed or Coulomb branch components. Quantum analysis is required to obtain the effective theory at or near the phase boundary. Simple cases will be treated in the next section.

Let us make a brief comment on the $\mathcal{N}=4$ systems. The vector multiplet includes an $\mathcal{N}=2$ chiral multiplet $\left(\sigma, i \bar{\lambda}_{+}\right)$in the adjoint, and the chiral multiplet includes an $\mathcal{N}=2$ fermi multiplet $\left(\psi_{-}, F\right)$ with $E=\sigma \phi$. Therefore, the scalar potential reads

$$
\begin{aligned}
U(\mathbf{x}, \phi) & =\frac{1}{2 e^{2}}\left|\left[x_{3}, \sigma\right]\right|^{2}+\left|x_{3} \phi\right|^{2}+\frac{e^{2}}{2}\left(\phi \bar{\phi}+\frac{1}{2 e^{2}}[\sigma, \bar{\sigma}]-\zeta\right)^{2}+|\sigma \phi|^{2}+|\mathrm{d} W(\phi)|^{2} \\
& =\frac{1}{2 e^{2}} \sum_{i<j}\left|\left[x_{i}, x_{j}\right]\right|^{2}+|\mathbf{x} \phi|^{2}+\frac{e^{2}}{2}(\phi \bar{\phi}-\zeta)^{2}+|\mathrm{d} W(\phi)|^{2}
\end{aligned}
$$

where we paid attention to the normalization $\frac{1}{2 e^{2}}$ of the kinetic term of $\left(\sigma, i \bar{\lambda}_{+}\right)$in (2.28). We also used $\zeta([\sigma, \bar{\sigma}])=0$ and $\left|\mathrm{x}_{3} \phi\right|^{2}+\frac{1}{2} \bar{\phi}[\sigma, \bar{\sigma}] \phi+|\sigma \phi|^{2}=|\mathbf{x} \phi|^{2}$ to go to the second line. Deep inside a phase where the gauge group is broken to a finite subgroup, the entire $\mathcal{N}=4$ vector multiplet together with the $\mathcal{N}=4$ chiral multiplet in the gauge orbit direction decouple from the low energy physics. In particular, we can set all three components of $\mathbf{x}$ to zero. This is the $\mathcal{N}=4$ Higgs branch. At the phase boundary, the vacuum manifold has mixed or Coulomb branch components. In each of them, the vanishing of the term $\left|\left[\mathrm{x}_{i}, \mathrm{x}_{j}\right]\right|^{2} / 2 e^{2}$ requires all three components of $\mathbf{x}$ to lie in the Lie algebra of a common maximal torus of the unbroken gauge group $G_{1}$.

Now, let us describe two typical phases - the geometric phase and the LandauGinzburg phase. There can also be hybrid of the geometric and Landau-Ginzburg phase.

\section{Geometric phase}

Suppose $G$ is completely broken, the set of solutions to the equations of (2.59)-(2.61) is a compact smooth manifold $\mathcal{C}_{\text {vac }}$, and the modes transverse to the solution space have non-zero masses. Then, as the effective description, we may take a non-linear sigma model whose target space is the quotient space $X=\mathcal{C}_{\text {vac }} / G$, which is a Kähler manifold. The sigma model may have fermions valued in a holomorphic vector bundle and/or a Wilson line determined by a complex of vector bundles.

The non-linear sigma model with a target Kähler manifold $(X, g)$ is described by a chiral multiplet $(\phi, \psi)$ where $\phi$ takes values in $X$ and $\psi$ takes values in the pull back $\phi^{*} T_{X}$

\footnotetext{
${ }^{3}$ In one dimension, the various fields and parameters have the following dimensions: $[D]=$ energy $^{2}$, $[\zeta D]=\left[D^{2} / e^{2}\right]=$ energy, and hence $[\zeta]=$ energy $^{-1}$ and $\left[e^{2}\right]=$ energy $^{3}$.
} 
( $T_{X}$ is the holomorphic tangent bundle of $X$ ). Its Lagrangian is

$$
L_{X}=g(\dot{\bar{\phi}}, \dot{\phi})+i g\left(\bar{\psi}, D_{t} \psi\right) \text {, }
$$

where $D_{t} \psi$ is the covariant derivative with respect to the pulled back Levi-Civita connection. The model may have a fermi multiplet $(\eta, F)$ with values in a holomorphic vector bundle with a hermitian metric $(\mathcal{E}, h)$ on $X$. Its Lagrangian is

$$
L_{\mathcal{E}}=i h\left(\bar{\eta}, D_{t} \eta\right)+h\left(\bar{\eta}, F_{A}(\psi, \bar{\psi}) \eta\right)+h(\overline{\tilde{F}}, \tilde{F})
$$

where $D_{t} \eta$ is the covariant derivative with respect to the pull back of the hermitian connection $A$ of $(\mathcal{E}, h), F_{A}(\psi, \bar{\psi}):=F_{i \bar{\jmath}} \psi^{i} \bar{\psi}^{\bar{\jmath}}$ with the curvature $F_{i \bar{\jmath}}$ of $A$, and $\tilde{F}:=F-\psi^{j} A_{j} \eta$ is the covariantized auxiliary field. See appendix A.3 for a more detailed description. The model may also have a Wilson line associated to a complex of vector bundles

$$
\cdots \stackrel{Q^{i-2}}{\longrightarrow} \mathcal{F}^{i-1} \stackrel{Q^{i-1}}{\longrightarrow} \mathcal{F}^{i} \stackrel{Q^{i}}{\longrightarrow} \mathcal{F}^{i+1} \stackrel{Q^{i+1}}{\longrightarrow} \cdots .
$$

Each $\mathcal{F}^{i}$ is equipped with a hermitian metric $h^{i}$ which determines a hermitian connection $A^{i}$. The Wilson line is valued in $\mathcal{E} n d(\mathcal{F}, \mathcal{F})$ with $\mathcal{F}=\oplus_{i} \mathcal{F}^{i}$ and is given by

$$
\mathcal{A}_{t}=-i\left(\phi^{*} A\right)_{t}-\frac{1}{2} F_{A}(\psi, \bar{\psi})-\psi^{i} \partial_{i} Q(\phi)+\bar{\psi}^{\bar{\tau}} \partial_{\bar{\imath}} Q(\phi)^{\dagger}+\left\{Q(\phi), Q(\phi)^{\dagger}\right\} .
$$

The construction is the same as in $2 d(2,2)$ theories with boundary. See [50] for detail.

The anomaly free condition is reflected in the effective description as the condition that the fermionic Hilbert space tensored with $\mathcal{F}$,

$$
\sqrt{K}_{X} \otimes \wedge T_{X} \otimes \operatorname{det}^{-\frac{1}{2}} \mathcal{E} \otimes \wedge \mathcal{E} \otimes \mathcal{F}
$$

forms a well-defined vector bundle on $X$. Note that $\mathcal{F}$ should not be a well-defined vector bundle if $\sqrt{K}_{X} \otimes \operatorname{det}^{-\frac{1}{2}} \mathcal{E}$ is not. The bundle $(2.68)$ is isomorphic to $\Omega_{X}^{0, \bullet} \otimes \mathfrak{F}$ with $\mathfrak{F}:=$ $\sqrt{K}_{X} \otimes \operatorname{det}^{-\frac{1}{2}} \mathcal{E} \otimes \wedge \mathcal{E} \otimes \mathcal{F}$ by the isomorphism $T_{X} \cong \bar{T}_{X}^{*}$.

The states of the system are sections of the vector bundle (2.68), or equivalently, antiholomorphic forms with values in $\mathfrak{F}$. As always in a theory with the supersymmetry (2.1), the space of supersymmetric ground states is isomorphic to the cohomology group of one of the supercharges, say $\overline{\mathbf{Q}}$. This operator acts on the states as

$$
i \overline{\mathbf{Q}} s=\bar{\partial} s+Q s .
$$

Thus, the space of supersymmetric ground states is

$$
\mathcal{H}_{\mathrm{SUSY}} \cong H_{\bar{\partial}+Q}^{0, \bullet}(X, \mathfrak{F})
$$

Since $X$ is compact, the Witten index is given by the Riemann-Roch formula

$$
I(\mathrm{id})=\int_{X} \operatorname{td}_{X} \mathrm{e}^{-\frac{1}{2} c_{1}(X)-\frac{1}{2} c_{1}(\mathcal{E})} \operatorname{ch}(\wedge \mathcal{E}) \operatorname{ch}(\mathcal{F}),
$$

where $\operatorname{ch}(\wedge \mathcal{E})=\sum_{i=0}^{\mathrm{rank} \mathcal{E}}(-1)^{i} \operatorname{ch}\left(\wedge^{i} \mathcal{E}\right)$ and $\operatorname{ch}(\mathcal{F})=\sum_{i}(-1)^{i} \operatorname{ch}\left(\mathcal{F}^{i}\right)$. 
Suppose $X$ admits a spin structure, $\mathcal{E}=0$, and $\mathcal{F}$ is just a vector bundle $\left(\mathcal{F}^{i}=0\right.$ for $i \neq 0)$. Then, $\sqrt{K}_{X} \otimes \wedge T_{X}$ makes sense as a vector bundle - it is the spinor bundle of $X$. Therefore, the states are spinors with values in $\mathcal{F}$. One can also show that the supercharge $\overline{\mathbf{Q}}+\mathbf{Q}$ is the Dirac operator. Indeed, (2.71) is the Atiyah-Singer formula for the Dirac index since $\operatorname{td}_{X} \mathrm{e}^{-\frac{1}{2} c_{1}(X)}=\widehat{\mathrm{A}}_{X}$.

The $\mathcal{N}=4$ supersymmetric non-linear sigma model with the target $(X, g)$ is obtained by setting $\mathcal{E}=T_{X}$ and $\mathcal{F}=0$. In this case, we have $\mathfrak{F}=K_{X} \otimes \wedge T_{X} \cong \wedge T_{X}^{*}$ with a shift of the $\mathbf{Z}_{2}$ grading by $n:=\operatorname{dim} X$. Hence the states are differential forms on $X$, and the space of supersymmetric ground states is the Dolbeault cohomology, which is isomorphic to the de Rham cohomology for the Kähler manifold,

$$
\mathcal{H}_{\mathrm{SUSY}} \cong \bigoplus_{p, q=0}^{n} H_{\bar{\partial}}^{0, q}\left(X, \wedge^{p} T_{X}^{*}[n]\right) \cong H_{\mathrm{de} \operatorname{Rham}}^{*}(X, \mathbf{C})[n]
$$

Vanishing of the flavor charge of the supersymmetric ground states is obvious in this representation, thanks to the identity $\mathcal{L}_{v}=d \circ i_{v}+i_{v} \circ d$ for a vector field $v$ on $X$. In fact, this is the origin of the general statement and its proof based on the identity (2.57), presented in section 2.1. The ground state for a $(p, q)$ class carries $2 J_{3}=p+q-n$ and $\boldsymbol{R}=-p+q$. If we take $(-1)^{F}=(-1)^{2 J_{3}}$, the canonically twisted index is

$$
I\left(\mathbf{y}^{2 \boldsymbol{J}_{-}}\right)=\sum_{p, q=0}^{n}(-1)^{p+q-n} \mathbf{y}^{-2 p+n} h^{p, q}(X)=\sum_{p=0}^{n}(-1)^{p-n} \mathbf{y}^{-2 p+n} \chi\left(X, \wedge^{p} T_{X}^{*}\right) .
$$

This is the $\chi_{y}$ genus of $X$ for $y=\mathbf{y}^{-2}$, up the prefactor $(-\mathbf{y})^{n}$. The untwisted index $I$ (id) is the Euler number $\chi(X)$ times $(-1)^{n}$. Indeed, $(2.71)$ is the Gauss-Bonnet formula since $\operatorname{td}_{X} \mathrm{e}^{-c_{1}(X)} \operatorname{ch}\left(\wedge T_{X}\right)=(-1)^{\operatorname{dim} X} e\left(T_{X}\right)$ which follows from $\frac{x}{1-e^{-x}} \mathrm{e}^{-x}\left(1-\mathrm{e}^{x}\right)=-x$.

We should note that one of the first applications of supersymmetry after [1] is the derivation of these index formulae via supersymmetric localization of the non-linear sigma models $[53,54]$.

\section{Landau-Ginzburg phase}

Suppose the vacuum equations (2.59)-(2.61) have a unique (up to gauge) solution at which the gauge group $G$ is broken to a finite group $\Gamma$. Then, as the effective theory, we can take the Landau-Ginzburg model of the massless variables modulo the gauge group $\Gamma$, that is, the Landau-Ginzburg orbifold.

A Landau-Ginzburg orbifold is obtained by the gauge theory described in the previous subsection by taking the gauge group to be the finite group $\Gamma$. In particular, there is no vector multiplet. The only rôle of the gauge group is the Gauss law, i.e., $\Gamma$ invariance of physical states, which makes sense when we can find $\operatorname{det}^{-\frac{1}{2}}\left(V_{\text {chiral }} \oplus V_{\text {fermi }}\right) \otimes M$ as a genuine representation of $\Gamma$. As remarked earlier, a fermi multiplet can be traded into a part of the Wilson line. Thus, we may consider only the chiral multiplet valued in $V_{\text {chiral }}$ with a Wilson line determined by $(M, \rho, Q)$. The anomaly free condition is then $\mathfrak{F}=\operatorname{det}^{-\frac{1}{2}} V_{\text {chiral }} \otimes M$ is a genuine representation of $\Gamma$. 
The states are $\Gamma$-invariant antiholomorphic differential forms on $V_{\text {chiral }}$ with values in $\mathfrak{F}$, and the supercharge $\overline{\mathbf{Q}}$ acts as (2.69). By the standard argument (see for example [50] section 2.2.3), the $\bar{\partial}+Q$ cohomology is isomorphic to the $Q$ cohomology acting on the space $\mathcal{H} o l\left(V_{\text {chiral }}, \mathfrak{F}\right)$ of holomorphic functions on $V_{\text {chiral }}$ with values in $\mathfrak{F}$. Thus, the space of supersymmetric ground states is

$$
\mathcal{H}_{\mathrm{SUSY}} \cong H_{Q}^{\bullet}\left(\mathcal{H} o l\left(V_{\text {chiral }}, \mathfrak{F}\right)\right)^{\Gamma}
$$

where the superscript $\Gamma$ stands for taking the $\Gamma$ invariants. When $(M, \rho, Q)$ comes from a fermi multiplet, as in (2.26) and (2.27), this is the untwisted and left-handed sector of the $2 d$ version of the system $[63,64]$.

The $\mathcal{N}=4$ Landau-Ginzburg orbifold with a $\Gamma$-invariant superpotential $W: V \rightarrow \mathbf{C}$ is obtained by taking $M=\operatorname{det}^{-\frac{1}{2}} V \otimes \wedge V$ and $\left.Q=-\mathrm{d} W\right\lrcorner$. In this case, we have an isomorphism $\mathfrak{F}=\operatorname{det}^{-1} V \otimes \wedge V \cong \wedge V^{*}$ (with a shift of $\mathbf{Z}_{2}$ grading by $n:=\operatorname{dim} V$ ) of $\Gamma$ representations, and $Q$ acts on $\mathcal{H o l}\left(V, \wedge V^{*}\right)$ by $-\mathrm{d} W \wedge$. The cohomology concentrates on the top degree forms and we have

$$
\mathcal{H}_{\mathrm{SUSY}} \cong\left(\frac{\mathcal{H o l}\left(V, \wedge^{n} V^{*}\right)}{\mathrm{d} W \wedge \mathcal{H o l}\left(V, \wedge^{n-1} V^{*}\right)}\right)^{\Gamma}[n] .
$$

This is nothing but the untwisted RR ground states in the $2 d$ version of the system [55].

\subsection{Examples}

\section{Example 1: projective space}

Let us first consider the $\mathcal{N}=2$ system with $\mathrm{U}(1)$ gauge group and $N$ chiral multiplets $\left(\phi_{1}, \psi_{1}\right), \ldots,\left(\phi_{N}, \psi_{N}\right)$ of charge 1 . We turn on the Wilson line $\mathcal{A}_{t}=\mathrm{q}\left(v_{t}+\sigma\right)$. The anomaly free condition is

$$
\frac{N}{2}+\mathrm{q} \in \mathbf{Z}
$$

The FI parameter $\zeta$ is naturally a real number and the D-term equation reads $\|\phi\|^{2}=\zeta$. When $\zeta$ is positive, the solution breaks the gauge group completely. The U(1) quotient of the space of solutions is the complex projective space $\mathbf{C} \mathbf{P}^{N-1}$. The modes transverse to the solution space are all massive and we are left with the non-linear sigma model whose target space is $\mathbf{C} \mathbf{P}^{N-1}$. Thus $\zeta>0$ is a geometric phase. The equation of motion sets $v_{t}$ to be the pull back of the gauge connection of the line bundle $\mathcal{O}(1)$ and therefore the Wilson line induces the "line bundle" $\mathcal{F}=\mathcal{O}(\mathrm{q})$. This yields $\mathfrak{F}=\mathcal{O}\left(\mathrm{q}-\frac{N}{2}\right)$, which is indeed a genuine line bundle over $\mathbf{C} \mathbf{P}^{N-1}$ by the anomaly free condition (2.76). The space of ground states of the effective sigma model is isomorphic to the Dolbeault cohomology

$\oplus_{i} H^{0, i}\left(\mathbf{C P}^{N-1}, \mathcal{O}\left(\mathrm{q}-\frac{N}{2}\right)\right)$. The dimension of each component is known to be

$$
\begin{aligned}
\mathrm{q} & \geq \frac{N}{2}: h^{0, i}\left(\mathbf{C P}^{N-1}, \mathcal{O}\left(\mathrm{q}-\frac{N}{2}\right)\right)= \begin{cases}0 & i \neq 0 \\
\left(\begin{array}{c}
\mathrm{q}+\frac{N}{2}-1 \\
N-1
\end{array}\right) & i=0,\end{cases} \\
-\frac{N}{2}<\mathrm{q}<\frac{N}{2}: \quad h^{0, i}\left(\mathbf{C P}^{N-1}, \mathcal{O}\left(\mathrm{q}-\frac{N}{2}\right)\right) & =0 \quad \forall i,
\end{aligned}
$$




$$
\mathrm{q} \leq-\frac{N}{2}: h^{0, i}\left(\mathbf{C P}^{N-1}, \mathcal{O}\left(\mathrm{q}-\frac{N}{2}\right)\right)= \begin{cases}0 & i \neq N-1 \\
\left(\begin{array}{ll}
\frac{N}{2}-\mathrm{q}-1 \\
N-1
\end{array}\right) & i=N-1\end{cases}
$$

When $\zeta$ is negative, there is no solution to the D-term equation, and we expect that there is no supersymmetric ground state.

Let us also consider the $\mathcal{N}=4$ system with $\mathrm{U}(1)$ gauge group and $N$ chiral multiplets $\left(\phi_{1}, \psi_{1 \pm}, F_{1}\right), \ldots,\left(\phi_{N}, \psi_{N \pm}, F_{N}\right)$ of charge 1 . No superpotential is allowed for this matter content, and there is no room for Wilson line in an $\mathcal{N}=4$ system as shown in section 2.1. The D-term equation is still $\|\phi\|^{2}=\zeta$, see (2.63). When $\zeta$ is positive, the solution breaks the gauge group completely. We are in the geometric phase where we have the $\mathcal{N}=4$ supersymmetric non-linear sigma model with the target space $\mathbf{C} \mathbf{P}^{N-1}$ as the effective description. The space of supersymmetric ground states is $\oplus_{p, q} H^{p, q}\left(\mathbf{C P}^{N-1}\right)$ whose dimension is

$$
h^{p, q}\left(\mathbf{C P}^{N-1}\right)=\left\{\begin{array}{l}
1 p=q=0, \ldots, N-1, \\
0 \text { otherwise }
\end{array}\right.
$$

When $\zeta$ is negative, there is no solution to the D-term equation, and we expect that the supersymmetry is broken.

\section{Example 2: hypersurface in projective space}

Let us consider the model which has a geometric phase and Landau-Ginzburg phase.

$\mathcal{N}=4$ model. We start with a much familiar system with $\mathcal{N}=4$ supersymmetry, with gauge group $\mathrm{U}(1), N$ chiral multiplets $\left(X_{1}, \psi_{1 \pm}, F_{1}\right), \ldots,\left(X_{N}, \psi_{N \pm}, F_{N}\right)$ of charge 1 , one chiral multiplet $\left(P, \psi_{P \pm}, F_{P}\right)$ of charge $-d$, and superpotential $W=\operatorname{Pf}(X)$, where $f(X)$ is a polynomial of degree $d$ which is generic in the sense that the common zero of $f(X)$ and its first derivatives is $X=0$ only. The D-term equation is $\|X\|^{2}-d|P|^{2}=\zeta$, while the F-term equations are $f(X)=0$ and $P \partial_{1} f(X)=\cdots=P \partial_{N} f(X)=0$.

When $\zeta$ is positive, the solution to the D-term equation has non-zero $X$ which breaks the gauge group completely. By the genericity of $f(X)$, the F-term equations require $P=f(X)=0$. Massless modes are modes of $X$ multiplets tangent to the vacuum manifold, which is the hypersurface $X_{f}=(f=0)$ of the projective space $\mathbf{C P} \mathbf{P}^{N-1}$. It is a geometric phase described effectively by the $\mathcal{N}=4$ sigma model whose target is $X_{f}$. The space of supersymmetric ground states is isomorphic to the cohomology group $\oplus_{p, q} H^{p, q}\left(X_{f}\right)$.

When $\zeta$ is negative, the solution to the $\mathrm{D}$-term equation has non-zero $P$ which breaks the gauge groups to $\mathbf{Z}_{d} \subset \mathrm{U}(1)$. By the genericity of $f(X)$, the solution to the vacuum equation is unique up to gauge: $P \neq 0, X=0$. Only the $X$ multiplets are massless, and we are left with the Landau-Ginzburg orbifold with superpotential $W(X)$ and orbifold group $\mathbf{Z}_{d}$.

$\mathcal{N}=\mathbf{2}$ model. We next consider a system with $\mathcal{N}=2$ supersymmetry. This is taken from the two-dimensional version [64] and shall be called the Distler-Kachru model. It is a $\mathrm{U}(1)$ gauge theory with $N$ chiral multiplets $\left(X_{1}, \psi_{1}\right), \ldots,\left(X_{N}, \psi_{N}\right)$ of charge 1 , one chiral multiplet $\left(P, \psi_{P}\right)$ of charge $-\ell$, one fermi multiplet $\left(\xi, F_{\xi}\right)$ of charge $-d, M$ chiral fermi multiplets $\left(\eta^{1}, F^{1}\right), \ldots,\left(\eta^{M}, F^{M}\right)$ of charge $q_{1}, \ldots, q_{M}$, and the superpotential $\mathfrak{W}=$ 


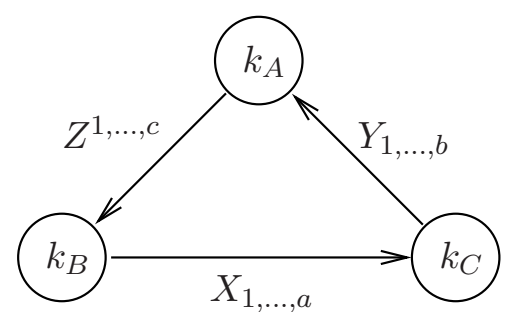

Figure 2. Triangle quiver.

$f(X) \xi+\sum_{\alpha} P g_{\alpha}(X) \eta^{\alpha}$, where $f(X), g_{1}(X), \ldots, g_{M}(X)$ are polynomials of degree $d, \ell-$ $q_{1}, \ldots, \ell-q_{M}$ which are generic in the sense that their common zero is $X=0$ only. (This requires $M+1 \geq N$.) We do not turn on the Wilson line. The anomaly free condition is

$$
N-\ell-d+\sum_{\alpha=1}^{M} q_{\alpha} \in 2 \mathbf{Z} .
$$

The model has a global $\mathrm{U}(1)_{F}$ symmetry under which $X, P, \xi, \eta$ have charges $01,0,-1$ respectively. The D-term equations are $\|X\|^{2}-\ell|P|^{2}=\zeta$ and the F-term equations are $f(X)=P g_{1}(X)=\cdots=P g_{M}(X)=0$.

When $\zeta$ is positive, the solution to the $\mathrm{D}$-term equations have non-zero $X$ which breaks the gauge group completely. The F-term equations require $P=f(X)=0$. Massless modes are modes of the $X$ multiplets tangent to the vacuum manifold $X_{f}$ as well as the modes of $\eta$ satisfying the equation $\sum_{\alpha} g_{\alpha}(X) \eta^{\alpha}=0$. It is a geometric phase described by the sigma model with the target $X_{f}$ and with the fermi multiplet valued in the kernel $\mathcal{E}$ of

$$
\bigoplus_{\alpha=1}^{M} \mathcal{O}\left(q_{\alpha}\right) \stackrel{\left(g_{\alpha}\right)_{\alpha=1}^{M}}{\longrightarrow} \mathcal{O}(\ell) .
$$

The space of states in this effective theory is the space of anti-holomorphic forms with values in $\mathfrak{F}=\sqrt{K}_{X_{f}} \otimes \operatorname{det}^{-\frac{1}{2}} \mathcal{E} \otimes \wedge \mathcal{E}=\mathcal{O}\left(-\frac{1}{2}\left(N-d+\sum_{\alpha=1}^{M} q_{\alpha}-\ell\right)\right) \otimes \wedge \mathcal{E}$ which is indeed a well-defined graded vector bundle over $X_{f}$ in view of (2.81).

When $\zeta$ is negative, the solution to the D-term equation has non-zero $P$ which breaks the gauge groups to $\mathbf{Z}_{\ell} \subset \mathrm{U}(1)$. The solution to the vacuum equation is unique up to gauge: $P \neq 0, X=0$. Massless modes are the chiral multiplet of $X$ and the chiral fermi multiplets of $\xi, \eta^{1}, \ldots, \eta^{M}$. The $\mathrm{U}(1)_{F}$ charges are $1 / \ell$ for $X,-d / \ell$ for $\xi$ and $-1+q_{\alpha} / \ell$ for $\eta^{\alpha}$ — these are obtained by dressing the original assignment with a gauge symmetry so that $P$ has charge zero. We are left with the Landau-Ginzburg orbifold of these variables with the superpotential $\mathfrak{W}=f(X) \xi+\sum_{\alpha} g_{\alpha}(X) \eta^{\alpha}$ and the orbifold group $\mathbf{Z}_{\ell}$.

\section{Example 3: quiver quantum mechanics}

An interesting class of examples with $\mathcal{N}=4$ supersymmetry is quiver quantum mechanics. Let us describe the phases for the triangle quiver as in figure 2. It has a gauge group $G=\left(\mathrm{U}\left(k_{A}\right) \times \mathrm{U}\left(k_{B}\right) \times \mathrm{U}\left(k_{C}\right)\right) / \mathrm{U}(1)_{\text {diag }}$, bifundamental matters as in figure 2 , and a 
generic superpotential of the form

$$
W=\sum_{i, j, h} A_{h}^{i j} \operatorname{tr}\left(Z^{h} Y_{j} X_{i}\right) .
$$

We parameterize the FI parameter as ${ }^{4}$

$$
\zeta(D)=\frac{\zeta^{2}}{k_{A}} \operatorname{tr}\left(D_{A}\right)-\frac{\zeta^{1}}{k_{B}} \operatorname{tr}\left(D_{B}\right)+\frac{\zeta^{1}-\zeta^{2}}{k_{C}} \operatorname{tr}\left(D_{C}\right) .
$$

The D-term and F-term equations read

$$
\begin{array}{lll}
Y Y^{\dagger}-Z^{\dagger} Z=\frac{\zeta^{2}}{k_{A}} \mathbf{1}_{k_{A}}, & \sum_{j, h} A_{h}^{i j} Z^{h} Y_{j}=0, & \forall i, \\
Z Z^{\dagger}-X^{\dagger} X=-\frac{\zeta^{1}}{k_{B}} \mathbf{1}_{k_{B}}, & \sum_{i, h} A_{h}^{i j} X_{i} Z^{h}=0, & \forall j, \\
X X^{\dagger}-Y^{\dagger} Y=\frac{\zeta^{1}-\zeta^{2}}{k_{C}} \mathbf{1}_{k_{C}}, & \sum_{i, j} A_{h}^{i j} Y_{j} X_{i}=0, & \forall h .
\end{array}
$$

where $X X^{\dagger}:=\sum_{i} X_{i} X_{i}^{\dagger}$ etc.

In order to find the phase structure, it is best to start looking for possible solutions to (2.85) with unbroken gauge symmetry. A typical unbroken subgroup is associated with a partition of the rank vector $\left(k_{A}, k_{B}, k_{C}\right)$ into two, $\left(k_{A}^{\prime}, k_{B}^{\prime}, k_{C}^{\prime}\right)+\left(k_{A}^{\prime \prime}, k_{B}^{\prime \prime}, k_{C}^{\prime \prime}\right)$, and includes the $\mathrm{U}(1)$ subgroup of elements of the form

$$
\left[\left(\begin{array}{cc}
z^{\prime} \mathbf{1}_{k_{A}^{\prime}} & 0 \\
0 & z^{\prime \prime} \mathbf{1}_{k_{A}^{\prime \prime}}
\end{array}\right),\left(\begin{array}{cc}
z^{\prime} \mathbf{1}_{k_{B}^{\prime}} & 0 \\
0 & z^{\prime \prime} \mathbf{1}_{k_{B}^{\prime \prime}}
\end{array}\right),\left(\begin{array}{cc}
z^{\prime} \mathbf{1}_{k_{C}^{\prime}} & 0 \\
0 & z^{\prime \prime} \mathbf{1}_{k_{C}^{\prime \prime}}
\end{array}\right)\right] .
$$

A solution invariant under this subgroup exists only when the FI parameter vanishes on its Lie algebra,

$$
\left(\frac{k_{C}^{\prime}}{k_{C}}-\frac{k_{B}^{\prime}}{k_{B}}\right) \zeta^{1}+\left(\frac{k_{A}^{\prime}}{k_{A}}-\frac{k_{C}^{\prime}}{k_{C}}\right) \zeta^{2}=0
$$

This is an empty condition when $k_{A}^{\prime} / k_{A}=k_{B}^{\prime} / k_{B}=k_{C}^{\prime} / k_{C}$, that is, when $k_{A}, k_{B}, k_{C}$ have a common divisor. In such a case, for any FI parameter, there is a solution with unbroken gauge group. In what follows, we assume that $k_{A}, k_{B}$ and $k_{C}$ are relatively prime. Then, the equation (2.87) defines a line in the FI-parameter space.

The line (2.87) is a candidate of the phase boundary. In many cases, however, only half of the line is the actual phase boundary, since the equations to be solved takes the form like $|\phi|^{2}=\zeta$ and has solution only for one sign of $\zeta$. It is also possible that a solution does not exists along both halves of the line. Obvious examples are $k_{A}^{\prime}$ and $k_{A}^{\prime \prime}$ both non-zero but $k_{B}^{\prime \prime}=k_{C}^{\prime}=0$. In such a case, $X, Y, Z$ must be of the form

$$
X=0, \quad Y=\left(\begin{array}{c}
0 \\
Y^{\prime \prime}
\end{array}\right), \quad Z=\left(\begin{array}{ll}
Z^{\prime} & 0
\end{array}\right)
$$

\footnotetext{
${ }^{4}$ It is an invariant linear form on $\mathfrak{g}=\mathfrak{u}\left(k_{A}\right) \oplus \mathfrak{u}\left(K_{B}\right) \oplus \mathfrak{u}\left(k_{C}\right) / \mathfrak{u}(1)_{\text {diag }}$, i.e., it must be invariant under conjugation and the shift of $\left(D_{A}, D_{B}, D_{C}\right)$ by $\left(\mathbf{1}_{k_{A}}, \mathbf{1}_{k_{B}}, \mathbf{1}_{k_{C}}\right)$. A general solution is (2.84).
} 


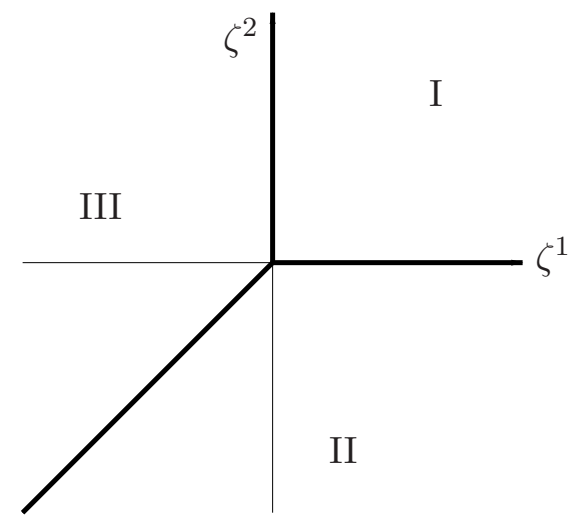

Figure 3. The phases of the model $(1,1,1)$.

and the D-term equations include $Y^{\prime} Y^{\prime \dagger}=\frac{\zeta^{2}}{k_{A}} \mathbf{1}_{k_{A}^{\prime}}$ and $-Z^{\prime \prime \dagger} Z^{\prime \prime}=\frac{\zeta^{2}}{k_{A}} \mathbf{1}_{k_{A}^{\prime \prime}}$, which have no solution if $\zeta \neq 0$. There are non-obvious examples where a solutions does not exist for some range of $(a, b, c)$ because the number of equations is too large compared to the number of variables.

In what follows, we present the phase structure of the model for some range of rank vectors. We assume that $a, b, c$ are all positive.

\section{Rank $(1,1,1)$}

The model has three phases as in figure 3 .

In Phase I, supersymmetry is unbroken if and only if $a+b-2 \geq c$, in which case we have the effective theory on the Higgs branch given by the non-linear sigma model on the complete intersection of the $c$ hypersurfaces $\sum_{i, j} A_{h}^{i j} X_{i} Y_{j}=0$ in $\mathbf{C P}^{a-1} \times \mathbf{C P}^{b-1}$. $Z^{h}=0$ there. The other phases are similar: to go from I to II and to III, we do the cyclic rotation $c \rightarrow b \rightarrow a$ and $Z \rightarrow Y \rightarrow X$. The three phases are separated by three phase boundaries. In the table below, we describe the unbroken gauge group $G_{1}$, the type of vacuum configuration, and the Higgs part of the mixed branch, at each of them:

$$
\begin{array}{|c|c|c|c|}
\hline & G_{1} & \text { type } & \text { Higgs part } \\
\hline \text { I-II } & \{[z, 1,1]\} \cong \mathrm{U}(1) & Y=Z=0 & \mathbf{C P}^{a-1} \\
\text { I-III } & \{[1, z, 1]\} \cong \mathrm{U}(1) & X=Z=0 & \mathbf{C P}^{b-1} \\
\text { II-III } & \{[1,1, z]\} \cong \mathrm{U}(1) & X=Y=0 & \mathbf{C P}^{c-1} \\
\hline
\end{array}
$$

\section{Rank $(k, 1,1)$}

The model has at most four phases as shown in figure 4 . When $b, c \geq k$, Cambers I and II are phases by themselves. Chambers III and IV combine into one phase when

$$
b \geq k, \quad c \geq k, \quad(b+c)(k-1) \leq a+(k-1)^{2},
$$

and are different phases otherwise. 


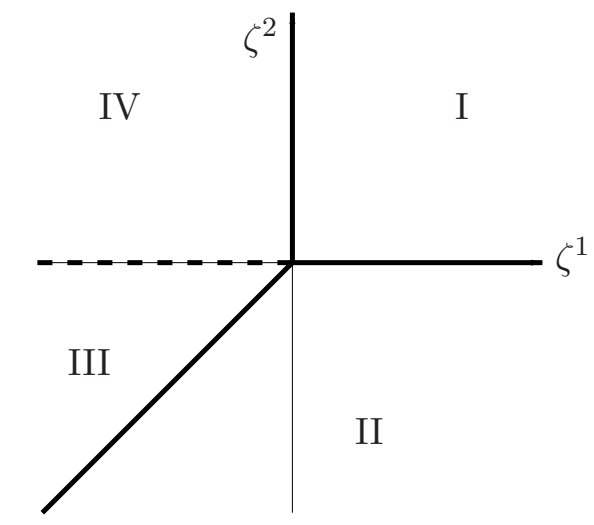

Figure 4. (Possible) phases of the model $(\mathrm{k}, 1,1)$.

In chamber I, supersymmetry is unbroken when $a \geq 1, b \geq k$ and $k(b-k)+a-1 \geq c k$, in which case we have the effective theory on the Higgs branch - the zero of the $c$ sections $\sum_{i, j} A_{h}^{i j} Y_{j} X_{i}$ of the rank $k$ vector bundle $S_{k, b}^{*} \otimes \mathcal{O}(1)$ over $G(k, b) \times \mathbf{C P}^{a-1}$. (Here $G(k, b)$ is the Grassmannian of $k$-planes in $\mathbf{C}^{b}$ and $S_{k, b}$ is its tautological vector bundle.) The description of chamber II is obtained from this by the exchange $Y \leftrightarrow Z, b \leftrightarrow c$. In chamber $\mathrm{IV}$, supersymmetry is unbroken when $b \geq k, c \geq 1$ and $k(b-k)+k c-1 \geq a$, in which case we have the effective theory on the Higgs branch - the complete intersection of $a$ hypersurfaces $\sum_{j, h} A_{h}^{i j} Z^{h} Y_{j}=0$ in $\mathbf{P}\left(S_{k, b}^{\oplus c}\right)$. Description in chamber III is obtained from this by the exchange $Y \leftrightarrow Z, b \leftrightarrow c$.

The Higgs branches in chambers IV and III are the same as complex manifolds if and only if the condition (2.90) is satisfied. To see that, let us regard $Y$ and $Z$ as $k \times b$ and $c \times k$ matrices. Then the defining F-term equations are $\sum_{j, h} A_{h}^{i j}(Z Y)^{h}{ }_{j}=0$ in both chambers. A possible difference is the condition on the rank of $Y$ and $Z$. In chamber IV we have $\operatorname{rank}(Y)=k$ and $\operatorname{rank}(Z) \leq k$ while in chamber III we have $\operatorname{rank}(Y) \leq k$ and $\operatorname{rank}(Z)=k$. The two manifolds are the same if $\operatorname{rank}(Z)$ is always $k$ in chamber IV and $\operatorname{rank}(Y)$ is always $k$ in chamber III. This obviously fails when $b$ or $c$ is less than $k$. But even when they are both bigger or equal to $k$, it fails when, say in chamber IV, there is a solution with $\operatorname{rank}(Z) \leq k-$ 1. Since $Y$ has rank $k$ there, this is equivalent to that the $c \times b$ matrix $Z Y$ has rank $(k-1)$ or less. This imposes $(c-(k-1))(b-(k-1))$ conditions on $Z Y$. On the other hand, the F-term equation $\sum_{j, h} A_{h}^{i j}(Z Y)_{j}^{h}=0$ imposes $a$ conditions on $Z Y$. Thus, there is such a solution when $b c-1 \geq(c-(k-1))(b-(k-1))+a$, which is the opposite of the last inequality in (2.90).

Accordingly, there are at most four phase boundaries. In the table below, we describe 
the data of the (possible) mixed branches:

\begin{tabular}{|c|c|c|c|}
\hline & $G_{1}$ & type & Higgs part \\
\hline I-II & $\{[g, 1,1]\} \cong \mathrm{U}(k)$ & $Y=Z=0$ & $\mathbf{C P}^{a-1}$ \\
I-IV & $\left\{\left[\mathbf{1}_{k}, z, 1\right]\right\} \cong \mathrm{U}(1)$ & $X=Z=0$ & $G(k, b)$ \\
II-III & $\left\{\left[\mathbf{1}_{k}, 1, z\right]\right\} \cong \mathrm{U}(1)$ & $X=Y=0$ & $G(k, c)$ \\
III-IV & $\left\{\left[\left(\begin{array}{rr}g \\
\mathbf{1}_{l}\end{array}\right), 1,1\right]\right\} \cong \mathrm{U}(k-l)$ & $Y=\left(\begin{array}{c}0 \\
Y^{\prime \prime}\end{array}\right), Z=\left(0 Z^{\prime \prime}\right)$ & $\mathcal{H}_{l}$ \\
\hline
\end{tabular}

The first three mixed branches exist when $a \geq 1, b \geq k$ and $c \geq k$ respectively. On the III-IV wall, $l$ ranges over $1, \ldots, k-1$. $\mathcal{H}_{l}$ is a variety of dimension $l(b+c)-l^{2}-1-a$ and is non-empty when this number is non-negative. For $l \geq 2$, it is singular if there is a locus where $\operatorname{rank}\left(Y^{\prime \prime}\right)=\operatorname{rank}\left(Z^{\prime \prime}\right)$ drops from $l$, and the singular locus is isomorphic to $\mathcal{H}_{l-1}$. ( $\mathcal{H}_{l}$ is smooth when $\mathcal{H}_{l-1}$ is empty.) $\mathcal{H}_{1}$ is always smooth and is given by the complete intersection of $a$ hypersurfaces $\sum_{j, h} A_{h}^{i j} z^{h} y_{j}=0$ in $\mathbf{C P}^{b-1} \times \mathbf{C P}^{c-1}$. The space of classical vacua is a union of the mixed branches,

$$
\mathcal{M}_{\text {III-IV }}=\bigcup_{l=1}^{k-1} \mathbf{R}^{3(k-l)} \times \mathcal{H}_{l} .
$$

Note that the $l$-th and the $(l-1)$-th mixed branches touch each other at the singular locus of $\mathcal{H}_{l}$ of the former and the origin of the $\mathbf{R}^{3}$ factor of $\mathbf{R}^{3(k-l+1)}$ of the latter. When $b, c \geq k$, $\mathcal{H}_{k-1}$ has the largest dimension among $\mathcal{H}_{l}$ 's. Therefore, the space (2.92) is non-empty if $\mathcal{H}_{k-1}$ is non-empty, and that is so when the last inequality of (2.90) is violated. Thus, III-IV wall is indeed a genuine phase boundary when the mixed branch exists there.

Special cases of this class of triangle quivers are studied in two dimensions in [56]: the cases are $(a, b, c)=(k n, n, n)$ where the Higgs branches are Calabi-Yau manifolds - in that paper, $k$ here is denoted as $(n-k)$. The above structure of mixed branches on the III-IV wall ( $2 \mathrm{~d}$ version) was worked out by $\mathrm{KH}$ and was conveyed to the authors of [56] who initially missed the possibility that the III-IV wall may not be a phase boundary. Among models with Calabi-Yau threefolds as the Higgs branches, an example where III-IV wall is not a phase boundary is $k=2$ and $(a, b, c)=(8,4,4)$.

\section{Higher ranks}

As the rank vector increases, the number of phases proliferates, as shown in figure 5 . This is because the lines (2.87) corresponding to different partitions of the rank vector are generically different. The case of rank vector $(k, 1,1)$ was special in that the partitions $(k, 1,1)=(k-l, 0,0)+(l, 1,1)$ all define the same line, which includes the III-IV wall. For example, for the case of rank vector $(k, 2,1)$, the partitions $(k-l, 1,0)+(l, 1,1)$ define different lines $\zeta^{1}=(2(k-l) / k) \zeta^{2}$, which appear, for example in $k=5$, as the four dashed segments in the south-west directions in figure 5-Left. Also, for higher ranks, it is more difficult to explicitly describe the Higgs branch in each phase as well as the mixed branches at each phase boundary. The index formula which we will derive in this paper will be 

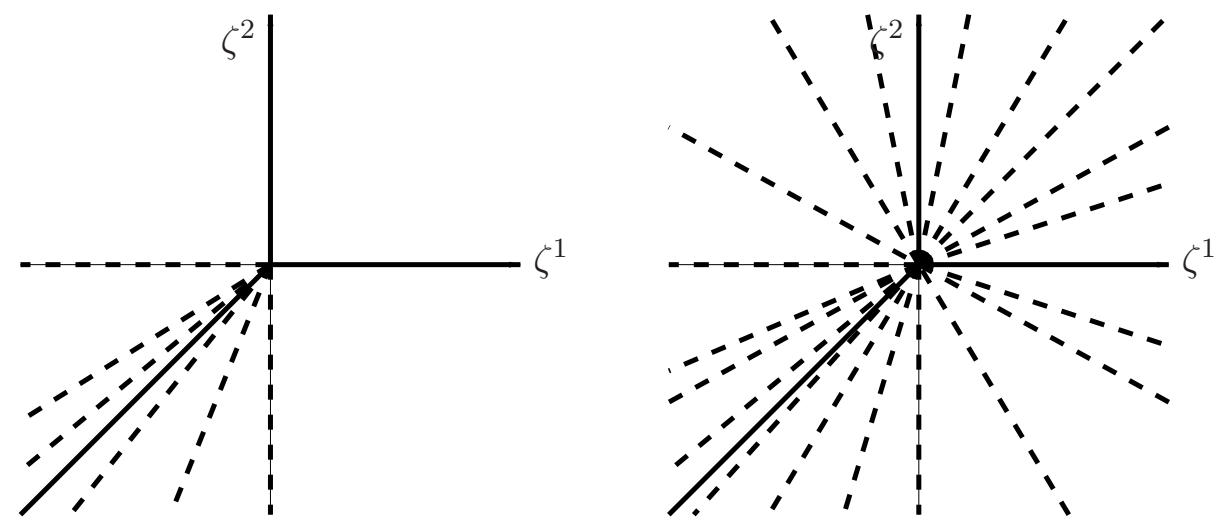

Figure 5. (Possible) phases of the models with $\vec{k}=(5,2,1)$ (Left) and $\vec{k}=(5,3,2)$ (Right).

particularly useful in such a circumstance, since we do not need to solve the D-term and F-term equations nor think about computing the cohomology on the space of solutions.

\section{Coulomb branch}

As we have seen, when the FI parameter $\zeta$ is deep inside a phase where the gauge group is broken to a finite subgroup, the Higgsed modes, consisting of the vector multiplet and the chiral multiplet in the gauge orbit direction, decouple from the physics below the energy scale $M_{H}=e \sqrt{|\zeta|}$. In this section, we would like to analyze what happens when the FI parameter $\zeta$ comes close to the walls.

For $\mathcal{N}=4$ systems, this was studied extensively, first by Denef [22] and later by many people including two of the authors of the present paper. As far as we are aware of, the analysis of $\mathcal{N}=2$ systems has not been done. As we shall see, they have much richer structures than $\mathcal{N}=4$ systems.

\subsection{The outline}

When the FI parameter $\zeta$ comes close to a wall, the mass of the Higgsed modes approaches zero. There, it is natural to look at the Coulomb branch - the region of the field space where the scalar component of the vector multiplet has large values. The chiral multiplet has a large mass in such a region and hence can be ignored, or to be more precise, should be integrated out. This leaves us with an effective theory of the vector multiplet. The main task is to determine this effective theory and then study its ground states.

We shall present the analysis for the theory with $\mathrm{U}(1)$ gauge group. We believe that this captures the essence of the general case, since typically only a single U(1) subgroup of the gauge group is unHiggsed at the wall. We shall examine later if that is indeed the case. Also, we shall start with the $\mathcal{N}=2$ systems, where the scalar $\sigma$ takes values in the real line. Note that there are two disconnected regions with "large $|\sigma|$ " — large positive $\sigma$ and large negative $\sigma$. The analysis should be done separately.

"Integrating out the matter chiral multiplet" is usually done by path-integral, where we can take the one-loop approximation for large values of $|\sigma|$. However, the operator 
formalism is more convenient in order to impose the Gauss law constraint. In that formulation, we shall find the ground state of the matter system in the background $(\sigma, \lambda, D)$ of the vector multiplet. The gauge field $v_{t}$ is used up already at this stage for the Gauss law. The matter theory has $\mathcal{N}=2$ supersymmetry in a supersymmetric background, which satisfies (see (2.4))

$$
\dot{\sigma}=D=0, \quad \lambda=0 .
$$

The matter system may or may not have a supersymmetric ground state. Correspondingly, the effective theory of the vector multiplet may or may not have a supersymmetry. It may happen that the matter system has a multiple of supersymmetric ground states. In that case, the effective theory decomposes into a multiple of sectors of supersymmetric theories. In general, the spectrum of matter ground states depends on whether $\sigma$ is large positive or large negative, and correspondingly the character of the effective theory of the vector multiplet depends on the regions of $\sigma$.

When the matter system preserves the supersymmetry, the effective Lagrangian must be supersymmetric. A supersymmetric Lagrangian of $(\sigma, \lambda, D)$ is of the form

$$
\frac{1}{2 e(\sigma)^{2}}\left(\dot{\sigma}^{2}+\frac{i}{2}(\bar{\lambda} \dot{\lambda}-\dot{\bar{\lambda}} \lambda)+D^{2}\right)-\left(\frac{1}{4 e(\sigma)^{2}}\right)^{\prime} D \bar{\lambda} \lambda+h^{\prime}(\sigma) D-\frac{1}{2} h^{\prime \prime}(\sigma) \bar{\lambda} \lambda
$$

to the second order in $\mathcal{O}(\sigma)=0, \mathcal{O}\left(\frac{\mathrm{d}}{\mathrm{d} t}\right)=1, \mathcal{O}(D)=1, \mathcal{O}(\lambda)=\frac{1}{2}$ and $\mathcal{O}(\epsilon)=-\frac{1}{2}$. (This order assignment is taken from [22].) The prime' stands for the $\sigma$ derivative. Note that the classical Lagrangian has $e(\sigma)=e$ and $h^{\prime}(\sigma)=-\zeta$. We know that the effective gauge coupling constant behaves at large $|\sigma|$ as $1 / e(\sigma)^{2}=1 / e^{2}+\# /|\sigma|^{3}+\cdots$, for some numerical constant \#. At large enough values, $|\sigma|^{3} \gg e^{2}$, we may ignore the correction and assume the following form of the effective Lagrangian,

$$
\begin{aligned}
L_{\mathrm{eff}} & =\frac{1}{2 e^{2}}\left(\dot{\sigma}^{2}+i \bar{\lambda} \dot{\lambda}+D^{2}\right)+h^{\prime}(\sigma) D-\frac{1}{2} h^{\prime \prime}(\sigma) \bar{\lambda} \lambda \\
& \simeq \frac{1}{2 e^{2}}\left(\dot{\sigma}^{2}+i \bar{\lambda} \dot{\lambda}\right)-\frac{e^{2}}{2}\left(h^{\prime}(\sigma)\right)^{2}-\frac{1}{2} h^{\prime \prime}(\sigma) \bar{\lambda} \lambda .
\end{aligned}
$$

When the matter system breaks the supersymmetry spontaneously, then the effective Lagrangian must have a supersymmetry breaking term. For example, the ground state energy of the matter sector, combined with the contribution from the Wilson line, is of the form $2 N_{B}|\sigma|$ for some positive integer $N_{B}$, so that the effective potential is

$$
U_{\text {eff }}(\sigma)=\frac{e^{2}}{2}\left(h^{\prime}(\sigma)\right)^{2}+2 N_{B}|\sigma| .
$$

The potential is positive and we do not expect to have a zero energy state. This only means that there is no supersymmetric ground state supported in the region of $\sigma$ under question. It is still possible that there are supersymmetric ground states supported on the other side of the $\sigma$ line or at $\sigma=0$.

Let us come back the case where the matter system preserves the supersymmetry. It turns out that the function $h^{\prime}(\sigma)$ is of the form

$$
h^{\prime}(\sigma)=\frac{N_{\mathrm{eff}}}{2|\sigma|}-\zeta
$$




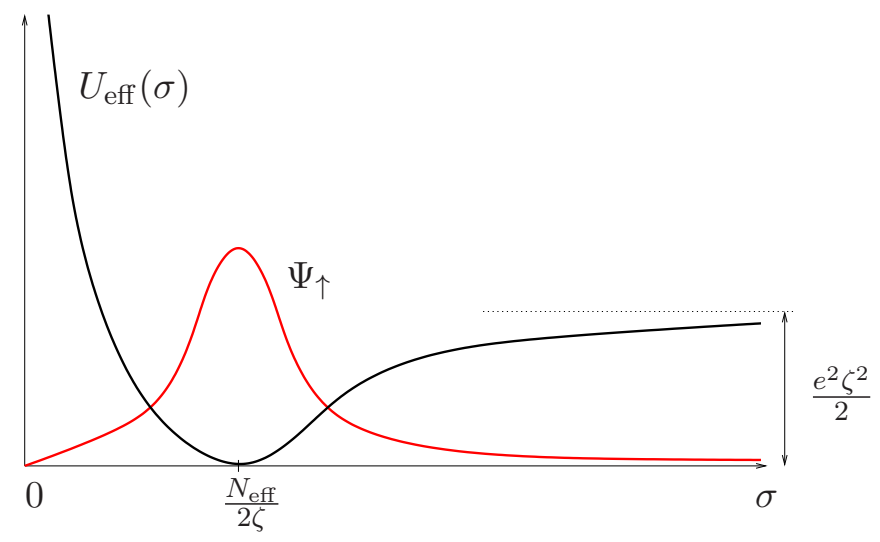

Figure 6. The effective potential and the wavefunction for the case $\zeta>0$ and $N_{\mathrm{eff}}>0$.

for an integer $N_{\text {eff }}$ that depends on the choice of the matter ground state. In particular, the effective potential is given by

$$
U_{\mathrm{eff}}(\sigma)=\frac{e^{2}}{2}\left(\frac{N_{\mathrm{eff}}}{2|\sigma|}-\zeta\right)^{2}
$$

Note that (3.3) is the famous system introduced in [1]. It is found that the exact supersymmetric ground state, if it exists, must be either of the following two,

$$
\Psi_{\downarrow}=\mathrm{e}^{-h(\sigma)}|0\rangle_{\text {eff }} \quad \text { or } \quad \Psi_{\uparrow}=\mathrm{e}^{h(\sigma)} \bar{\lambda}|0\rangle_{\mathrm{eff}},
$$

where $|0\rangle_{\text {eff }}$ is the state annihilated by $\lambda$. Which (or none) to be taken is decided by the requirement that the wavefunction is square normalizable. Integrating (3.5) we find

$$
\mathrm{e}^{h(\sigma)}=|\sigma|^{\frac{N_{\text {eff }}}{2} \operatorname{sgn}(\sigma)} \mathrm{e}^{-\zeta \sigma} .
$$

Suppose we have the supersymmetric effective theory (3.3) with (3.5) on the $\sigma>0$ Coulomb branch. If $\zeta>0$, we should take $\Psi_{\uparrow}$ for square normalizability at $\sigma \rightarrow \infty$. In addition, if $N_{\text {eff }}>0$, then the wavefunction $\Psi_{\uparrow}$ vanishes as $\sigma \rightarrow 0$. It is square normalizable over $\sigma>0$ and is supported around $\sigma=N_{\text {eff }} / 2 \zeta$. (See figure 6) Thus, it qualifies as the supersymmetric ground state on the $\sigma>0$ Coulomb branch. If $N_{\text {eff }}<0$ on the other hand, the wavefunction $\Psi_{\uparrow}$ diverges as $\sigma \rightarrow 0$ and is not square normalizable over $\sigma>0$. That it is not square normalizable at $\sigma \rightarrow 0$ may not be a serious problem since we are interested in large values of $|\sigma|$ anyway. However, the fact that its support is toward $\sigma \rightarrow 0$ disqualifies it to be a Coulomb branch vacuum. Subtle is the case $N_{\text {eff }}=0$ : the state $\Psi_{\uparrow}$ does not diverge as $\sigma \rightarrow 0$ and is perfectly square normalizable over $\sigma>0$, but the peak of the support is toward $\sigma \rightarrow 0$. Therefore it is not clear at this point if we should discard it or not. If $\zeta<0$, we should take $\Psi_{\downarrow}$, and it qualifies as a supersymmetric ground state on the $\sigma>0$ Coulomb branch only when $N_{\text {eff }}<0$. Similarly for the case where we have the supersymmetric effective theory (3.3) with (3.5) on the $\sigma<0$ Coulomb branch. If $\zeta>0$ (resp. $\zeta<0$ ), we should take $\Psi_{\downarrow}\left(\right.$ resp. $\left.\Psi_{\uparrow}\right)$ and it qualifies as a supersymmetric ground state on the $x<0$ Coulomb branch if $N_{\text {eff }}>0$ (resp. $\left.N_{\text {eff }}<0\right)$. Subtle is the case $N_{\text {eff }}=0$ where it is normalizable but has a profile which is peaked toward the origin $\sigma \rightarrow 0$. 
Let us see what happens to the ground states as the FI parameter $\zeta$ is varied across zero, say from positive to negative. For concreteness, we consider the $\sigma>0$ Coulomb branch. First suppose $N_{\text {eff }}>0$. As we have seen, for $\zeta>0$, there is a supersymmetric ground state $\Psi_{\uparrow}$ which is supported around $\sigma=N_{\text {eff }} / 2 \zeta$. As $\zeta$ approaches 0 from above, the location of the support runs away to infinity. For $\zeta<0$, there is no supersymmetric ground state on the $\sigma>0$ Coulomb branch. This is the wall crossing phenomenon - a normalizable supersymmetric ground state disappears by exiting the field space at infinity. When $N_{\text {eff }}<0$, the opposite happens: there is no supersymmetric ground state for $\zeta>0$. As $\zeta$ crosses zero and goes negative, a supersymmetric ground state $\Psi_{\downarrow}$ enters from infinity. Subtle is the case $N_{\text {eff }}=0$ : there is a square normalizable supersymmetric ground state before and after $\zeta$ crosses zero $-\Psi_{\uparrow}$ for $\zeta>0$ and $\Psi_{\downarrow}$ for $\zeta<0$. As $\zeta \rightarrow 0$, its profile does not run away to infinity but instead spreads out. As we will see in examples, it does play a particular rôle in the wall crossing phenomenon.

The effective theory on the Coulomb branch has a characteristic energy scale

$$
M_{C}=e^{2} \zeta^{2}
$$

To show this, let us rescale the fields as $\sigma \rightarrow e \sigma$ and $\lambda \rightarrow e \lambda$ so that they have the canonically normalized kinetic terms. Then, the effective Lagrangian depends on $e$ and $\zeta$ only through the combination $e \zeta$ which has the dimension of $\sqrt{\text { energy }}$. It depends also on $N_{\text {eff }}$ and possibly also $N_{B}$ but they are integer valued discrete parameters. All relevant energy scale is $M_{C}$ times a numerical constant. Indeed, the hight of the potential $U_{\text {eff }}$ at $|\sigma| \rightarrow \infty$ is $e^{2} \zeta^{2} / 2$ (in the supersymmetric region). Also, the perturbative spectrum around the supersymmetric ground state $\Psi_{\uparrow}$ discussed above has level spacing $\omega=2 e^{2} \zeta^{2} / N_{\text {eff }}$ since the potential behaves near the bottom $\sigma=\frac{N_{\text {eff }}}{2 \zeta}+e \xi$ as $U_{\text {eff }} \simeq \frac{\omega^{2}}{2} \xi^{2}$.

Let us now describe the Coulomb branch of the $\mathcal{N}=4$ systems with a $\mathrm{U}(1)$ gauge group. Everything is essentially obtained in [22]. We would like to find the effective theory of the variables $(\mathbf{x}, \boldsymbol{\lambda}, \boldsymbol{D})$ at large values of $|\mathbf{x}|$. The matter sector always has a unique supersymmetric ground state in the supersymmetric backgrounds, and yields a supersymmetric Lagrangian for $(\mathbf{x}, \boldsymbol{\lambda}, \boldsymbol{D})$. It must be of the form

$$
\boldsymbol{L}_{\mathrm{eff}}=\frac{1}{2 e^{2}}\left(\dot{\mathbf{x}}^{2}+i \overline{\boldsymbol{\lambda}} \dot{\boldsymbol{\lambda}}+\boldsymbol{D}^{2}\right)-V(\mathbf{x}) \boldsymbol{D}-\boldsymbol{A}(\mathbf{x}) \cdot \dot{\mathbf{x}}+\boldsymbol{C}(\mathbf{x}) \cdot \bar{\lambda} \boldsymbol{\sigma} \boldsymbol{\lambda}
$$

with $\boldsymbol{C}=\frac{1}{2} \nabla V=-\frac{1}{2} \nabla \times \boldsymbol{A}$, to the second order in $\mathcal{O}$, if we neglect terms that vanishes in the regime $e^{2} /|\mathbf{x}|^{3} \ll 1$. An explicit computation, say in path-integral, yields

$$
V=\zeta-\frac{N_{\mathrm{eff}}^{(4)}}{2|\mathbf{x}|}, \quad \boldsymbol{A}=N_{\mathrm{eff}}^{(4)} \boldsymbol{A}_{\mathrm{mono}}, \quad \boldsymbol{C}=\frac{N_{\mathrm{eff}}^{(4)}}{4} \frac{\mathbf{x}}{|\mathbf{x}|^{3}},
$$

where

$$
N_{\mathrm{eff}}^{(4)}:=\#(\text { positively charged fields) - \#(negatively charged fields), }
$$

and $\boldsymbol{A}_{\text {mono }}$ is the Dirac monopole of unit magnetic charge. In particular, $\boldsymbol{A}$ has first Chen class $N_{\text {eff }}^{(4)}$ on the sphere surrounding the origin. The four supercharges are proportional 
to ${ }^{5}$

$$
\overline{\boldsymbol{\lambda}}\left(\boldsymbol{\sigma} \cdot \boldsymbol{D}_{\boldsymbol{A}}-V\right), \quad\left(\boldsymbol{\sigma} \cdot \boldsymbol{D}_{\boldsymbol{A}}+V\right) \boldsymbol{\lambda},
$$

where $\boldsymbol{D}_{\boldsymbol{A}}$ is the covariant derivative, $\left(D_{\boldsymbol{A}}\right)_{j}=\partial_{j}+i A_{j}$, for $\boldsymbol{A}$ in (3.11). Note that the $\mathrm{SU}(2) \times \mathrm{U}(1)$ R-symmetry is present in this effective theory, since the background $(V, \boldsymbol{A}$, $\boldsymbol{C})$ is rotationally symmetric. $\mathrm{U}(1)_{R}$ is just a phase rotation of $\boldsymbol{\lambda}$ and $\overline{\boldsymbol{\lambda}}$.

The supersymmetric ground states are of the form

$$
\Psi^{\alpha}(\mathbf{x}) \bar{\lambda}_{\alpha}|0\rangle_{\text {osc }},
$$

for $\Psi(\mathbf{x})$ satisfying

$$
\left(\boldsymbol{\sigma} \cdot \boldsymbol{D}_{\boldsymbol{A}}+V\right) \Psi(\mathbf{x})=0 .
$$

The doublet wavefunction $\Psi(\mathbf{x})$ is a spinor on $\mathbf{R}^{3} \backslash 0$ with values in a line bundle of magnetic charge $N_{\text {eff }}^{(4)}$. We may regard it as a spinor on $S^{2}$ with values in $\mathcal{O}\left(N_{\text {eff }}^{(4)}\right)$ which depends on $r=|\mathbf{x}|$. With respect to the chiral basis on $S^{2}$, the vacuum equation (3.15) takes the form

$$
\left\{\left(\begin{array}{cc}
\partial_{r} & 0 \\
0 & -\partial_{r}
\end{array}\right)+\frac{1}{r}\left[\left(\begin{array}{cc}
1 & 0 \\
0 & -1
\end{array}\right)+\boldsymbol{D}_{S^{2}, \boldsymbol{A}}\right]+V\right\}\left(\begin{array}{l}
\Psi_{-} \\
\Psi_{+}
\end{array}\right)=0 .
$$

$\Psi_{ \pm}$is the left/right component of $\Psi$, i.e., an $r$-dependent section of the line bundle $\mathcal{O}\left(N_{\mathrm{eff}}^{(4)} \pm\right.$ 1). $\boldsymbol{D}_{S^{2}, \boldsymbol{A}}$ is the Dirac operator on $S^{2}$ which swaps the left and the right components. We may decompose $\Psi$ into eigenmodes of $\boldsymbol{D}_{S^{2}, \boldsymbol{A}}$. A non-zero mode has both the left and the right components and hence cannot solve (3.16). A zero mode has only the left or the right component and can solve (3.16). Recall the index theorem

$$
\text { ind } \boldsymbol{D}_{S^{2}, \boldsymbol{A}}=N_{\mathrm{eff}}^{(4)} \text {. }
$$

In fact the index is the actual number of zero modes. When $N_{\text {eff }}^{(4)}$ is zero, there is no zero mode. In particular, there is no supersymmetric ground state for any value of $\zeta$. When $N_{\text {eff }}^{(4)}$ is positive, $\Psi_{-}$has $N_{\text {eff }}^{(4)}$ zero modes which may be represented by the holomorphic sections of $\mathcal{O}\left(N_{\mathrm{eff}}^{(4)}-1\right)$. They form the spin $j=\frac{N_{\mathrm{eff}}^{(4)}-1}{2}$ representation of $\mathrm{SU}(2)$ (Borel-Weil theorem). The equation (3.16) reads

$$
\left[\frac{\partial}{\partial r}+\frac{1}{r}+\zeta-\frac{N_{\mathrm{eff}}^{(4)}}{2 r}\right] \Psi_{-}=0 .
$$

For $\zeta>0$, it has a unique solution that vanishes at infinity, $\Psi_{-} \propto r^{\frac{N_{\text {eff }}^{(4)}}{2}-1} \mathrm{e}^{-\zeta r}$. Note that

$$
r^{2}\left|\Psi_{-}\right|^{2} \propto\left(r^{\frac{N_{\text {eff }}^{(4)}}{2}} \mathrm{e}^{-\zeta r}\right)^{2}
$$

which shows that it is square normalizable. This also shows that the probability to be in the shell of radius $r$ is largest for $r=N_{\text {eff }}^{(4)} /(2 \zeta)$. As $\zeta \searrow 0$, the peak runs away to

\footnotetext{
${ }^{5}$ There is a sign error in [22].
} 
infinity, and for $\zeta<0$ there is no normalizable solution. Therefore, when $N_{\mathrm{eff}}^{(4)}$ is positive, $N_{\text {eff }}^{(4)}$ supersymmetric ground states forming the spin $j=\frac{N_{\text {eff }}^{(4)}-1}{2}$ representation of $\mathrm{SU}(2)$ run away to infinity in $|\mathbf{x}|$ as $\zeta$ is sent from positive to negative. When $N_{\text {eff }}^{(4)}$ is negative, $\Psi_{+}$has $\left|N_{\text {eff }}^{(4)}\right|$ zero modes represented by anti-holomorphic sections of $\mathcal{O}\left(N_{\text {eff }}^{(4)}+1\right)$. The equation (3.16) reads

$$
\left[-\frac{\partial}{\partial r}-\frac{1}{r}+\zeta-\frac{N_{\mathrm{eff}}^{(4)}}{2 r}\right] \Psi_{+}=0 .
$$

For $\zeta>0$, there is no normalizable solution. For $\zeta<0$, there is a unique normalizable solution such that

$$
r^{2}\left|\Psi_{+}\right|^{2} \propto\left(r^{-\frac{N_{\text {eff }}^{(4)}}{2}} \mathrm{e}^{\zeta r}\right)^{2} .
$$

Note that the peak $r=N_{\mathrm{eff}}^{(4)} /(2 \zeta)$ runs off to infinity as $\zeta \nearrow 0$. Therefore, when $N_{\mathrm{eff}}^{(4)}$ is negative, $\left|N_{\mathrm{eff}}^{(4)}\right|$ supersymmetric ground states forming the spin $j=\frac{\left|N_{\mathrm{eff}}^{(4)}\right|-1}{2}$ representation of $\mathrm{SU}(2)$ come in from infinity in $|\mathbf{x}|$ as $\zeta$ is sent from positive to negative.

The above ground states, whose wavefunctions are of the form (3.14), have vanishing $\mathrm{U}(1) \mathrm{R}$-charge. This is because only $\boldsymbol{\lambda}$ and $\overline{\boldsymbol{\lambda}}$ have nonzero R-charge, and the conjugate invariant quantization yields R-charge $1,0,-1$ on the states $|0\rangle_{\text {osc }}, \bar{\lambda}_{ \pm}|0\rangle_{\text {osc }}, \bar{\lambda}_{+} \bar{\lambda}_{-}|0\rangle_{\text {osc }}$, respectively. In view of the relation between $\boldsymbol{J}_{ \pm}$and the generators of $\mathrm{SU}(2) \times \mathrm{U}(1)_{R}$ (see the footnote in page 14), we see that these ground states have $\boldsymbol{J}_{ \pm}= \pm J_{3}$.

In what follows, we illustrate these results in some of the examples introduced in section 2.4.

\subsection{The $\mathrm{CP}^{N-1}$ model}

Let us look into the detail of the Coulomb branch of the $\mathbf{C P}^{N-1}$ model (Example 1). First, the $\mathcal{N}=2$ supersymmetric system: $\mathrm{U}(1)$ gauge group, $N$ chiral multiplets of charge 1 , and Wilson line of charge $q$, obeying the anomaly free condition (2.76), $N / 2+\mathrm{q} \in \mathbf{Z}$.

The Lagrangian of the system is

$$
\begin{aligned}
L= & \frac{1}{2 e^{2}}\left(\dot{\sigma}^{2}+i \bar{\lambda} \dot{\lambda}+D^{2}\right)-\zeta D-\mathrm{q}\left(v_{t}+\sigma\right) . \\
& +\left|D_{t} \phi\right|^{2}+i \bar{\psi} D_{t} \psi+\bar{\phi}\left(D-\sigma^{2}\right) \phi+\bar{\psi} \sigma \psi-i \bar{\phi} \lambda \psi+\bar{\psi} \bar{\lambda} \phi .
\end{aligned}
$$

We first quantize the matter system, with the Lagrangian on the second line, in the supersymmetric background (3.1), i.e., $D=\lambda=0$ and $\sigma$ constant. Later, fluctuations away from the background shall be taken into account as perturbation. This matter system is a free theory and we know the exact spectrum. Let us denote by $a_{\phi}$ and $a_{\bar{\phi}}$ (resp. $a_{\phi}^{\dagger}$ and $a_{\bar{\phi}}^{\dagger}$ ) the annihilation (resp. creation) operators of $\phi$ and $\bar{\phi}$. The Hamiltonian and the gauge charge are

$$
\begin{aligned}
H_{\text {matter }} & =\omega\left(a_{\phi}^{\dagger} a_{\phi}+a_{\frac{\phi}{\phi}}^{\dagger} a_{\bar{\phi}}+N\right)-\sigma \frac{[\bar{\psi}, \psi]}{2}, \\
\mathbf{G}_{\text {matter }} & =a_{\phi}^{\dagger} a_{\phi}-a_{\frac{\phi}{\phi}}^{\dagger} a_{\bar{\phi}}+\frac{[\bar{\psi}, \psi]}{2} .
\end{aligned}
$$


The oscillator frequency is $\omega=|\sigma|$ for now - it will be replaced by $\omega=\sqrt{\sigma^{2}-D}$ when the fluctuation is introduced. A general state is of the form

$$
|n, \bar{n}, m\rangle=\left(\prod_{i=1}^{N} \frac{a_{\phi_{i}}^{\dagger n_{i}}}{\sqrt{n_{i} !}} \frac{a_{\bar{\phi}_{i}}^{\dagger \bar{n}_{i}}}{\sqrt{\bar{n}_{i} !}} \bar{\psi}_{i}^{m_{i}}\right)|0\rangle_{\mathrm{osc}}
$$

which has energy and charge

$$
\begin{aligned}
& E_{\text {matter }}=\omega(|n|+|\bar{n}|+N)-\sigma\left(|m|-\frac{N}{2}\right), \\
& Q_{\text {matter }}=|n|-|\bar{n}|+|m|-\frac{N}{2} .
\end{aligned}
$$

In the above expressions, $|0\rangle_{\text {osc }}$ is the state annihilated by $a_{\phi_{i}}, a_{\bar{\phi}_{i}}$ and $\psi_{i}$, and we define $|n|:=\sum_{i=1}^{N} n_{i}$, etc. Note that $n_{i}$ and $\bar{n}_{i}$ run over all non-negative integers while $m_{i}$ runs over 0 and 1 . In particular, $|m|$ can range only over $0,1, \ldots, N$. The Gauss law is $\left(\mathbf{G}_{\text {matter }}+\mathrm{q}\right) \mid$ phys $\rangle=0$, which requires the state (3.25) to satisfy

$$
|n|-|\bar{n}|+|m|-\frac{N}{2}+\mathrm{q}=0
$$

Including the linear potential q $\sigma$ from the Wilson line, the state has energy

$$
\begin{aligned}
E & =\omega(|n|+|\bar{n}|+N)-\sigma\left(|m|-\frac{N}{2}-\mathrm{q}\right) \\
& = \begin{cases}2|\sigma|\left(|n|+\frac{N}{2}+\mathrm{q}\right)=2|\sigma|(|\bar{n}|+(N-|m|)) & \text { for } \sigma>0, \\
2|\sigma|\left(|\bar{n}|+\frac{N}{2}-\mathrm{q}\right)=2|\sigma|(|n|+|m|) & \text { for } \sigma<0 .\end{cases}
\end{aligned}
$$

The energy is indeed non-negative if the Gauss law is taken into account. It is zero (i.e., the state is supersymmetric) when:

$$
\begin{aligned}
& \sigma>0: \quad|\bar{n}|=0, \quad|m|=N, \quad|n|=-\mathrm{q}-\frac{N}{2}, \\
& \sigma<0: \quad|n|=0, \quad|m|=0, \quad|\bar{n}|=\mathrm{q}-\frac{N}{2} .
\end{aligned}
$$

Since $|n|$ and $|\bar{n}|$ are non-negative, supersymmetric ground states exist at $\sigma>0$ (resp. $\sigma<0)$ if and only if $\mathrm{q} \leq-\frac{N}{2}$ (resp. $\mathrm{q} \geq \frac{N}{2}$ ). The degeneracy is the number of $n$ 's with $|n|=-\mathrm{q}-\frac{N}{2}$ (resp. $\bar{n}$ 's with $|\bar{n}|=\mathrm{q}-\frac{N}{2}$ ), which is

$$
\left(\begin{array}{c}
-\mathrm{q}-\frac{N}{2}+N-1 \\
N-1
\end{array}\right) \quad\left(\operatorname{resp} . \quad\left(\begin{array}{c}
\mathrm{q}-\frac{N}{2}+N-1 \\
N-1
\end{array}\right)\right) .
$$

When this condition is violated, i.e. on $\sigma>0$ for $\frac{N}{2}+q>0$ (resp. on $\sigma<0$ for $\frac{N}{2}-q>0$ ), supersymmetry is spontaneously broken. The ground states are those with $|n|=0$ (resp. $|\bar{n}|=0$ ) having energy

$$
E_{0}=2|\sigma|\left(\frac{N}{2}+\mathrm{q}\right) \quad\left(\text { resp. } 2|\sigma|\left(\frac{N}{2}-\mathrm{q}\right)\right) .
$$


There is a degeneracy with multiple possibilities for $|\bar{n}|=|m|-N+\left(\frac{N}{2}+\mathrm{q}\right)$ (resp. $|n|=$ $\left.-|m|+\left(\frac{N}{2}-\mathrm{q}\right)\right)$.

Let us now take into account the fluctuation from the supersymmetric background (3.1) as a perturbation. We describe in detail the region of $\sigma$ where the unperturbed system has supersymmetric ground states. The effect of non-zero $D$ simply occurs in $\omega=\sqrt{\sigma^{2}-D}=$ $|\sigma|-\frac{D}{2|\sigma|}+\cdots$. To the first order in $D$, it shifts the ground state energy as

$$
\begin{aligned}
\Delta_{D} E_{0} & =-(|n|+|\bar{n}|+N) \frac{D}{2|\sigma|} \\
& =-\left(\frac{N}{2}+|\mathrm{q}|\right) \frac{D}{2|\sigma|},
\end{aligned}
$$

where we used (3.30)/(3.31). The effect of non-zero $\lambda$ is the perturbation $\Delta_{\lambda} H=i \bar{\phi} \lambda \psi-$ $i \bar{\psi} \bar{\lambda} \phi=-\frac{1}{\sqrt{2 \omega}}\left(a_{\bar{\phi}}-a_{\phi}^{\dagger}\right) \lambda \psi+\frac{1}{\sqrt{2 \omega}} \bar{\psi} \bar{\lambda}\left(a_{\phi}-a_{\bar{\phi}}^{\dagger}\right)$, which can be treated in the standard way. Since all the ground states have the same $|m|$ and $\Delta_{\lambda} H$ changes the $\psi$ number, the energy shift vanishes to the first order. The second order shifts are the eigenvalues of the matrix

$$
A_{i j}=\sum_{m \neq 0} \frac{\left(\Delta_{\lambda} H\right)_{0_{i} m}\left(\Delta_{\lambda} H\right)_{m 0_{j}}}{0-E_{m}}
$$

where $\left\{0_{i}\right\}$ are the basis of the ground states and $m$ runs over the excited states, in the unperturbed system. In fact it is diagonal with all the same eigenvalue,

$$
\Delta_{\lambda} E_{0}=\frac{1}{0-2|\sigma|}\left\{\begin{array}{ll}
\frac{1}{2 \omega} \bar{\lambda} \lambda \sum_{i}\left(n_{i}+1\right) & (\sigma>0) \\
\frac{1}{2 \omega} \lambda \bar{\lambda} \sum_{i}\left(\bar{n}_{i}+1\right) & (\sigma<0)
\end{array}=-\left(\frac{N}{2}+|\mathrm{q}|\right) \frac{\operatorname{sgn}(\sigma)}{4 \sigma^{2}} \bar{\lambda} \lambda\right.
$$

where $(3.30) /(3.31)$ is used again. To summarize, to the first non-trivial order, the effect of perturbation is the shift of the ground state energy by

$$
\Delta E=-\left(\frac{N}{2}+|\mathrm{q}|\right) \operatorname{sgn}(\sigma)\left[\frac{D}{2 \sigma}+\frac{1}{4 \sigma^{2}} \bar{\lambda} \lambda\right] .
$$

When the unperturbed system breaks the supersymmetry, the effect of non-zero $D$ is similar to (3.34) though the coefficient $|n|+|\bar{n}|+N$ depends on the choice of the degenerate vacua. The effect of non-zero $\lambda$ is far more complicated to explain here.

Integrating out the auxiliary field $D$, we find that the effective potential is of the form

$$
U_{\mathrm{eff}}(\sigma)=\frac{e^{2}}{2}\left(\frac{N_{\mathrm{eff}}}{2|\sigma|}-\zeta\right)^{2}+2 N_{B}|\sigma|
$$

$N_{B}$ is read from (3.29) and (3.33). In the region $\sigma>0$ for $\mathrm{q} \leq-\frac{N}{2}$ and $\sigma<0$ for $\mathrm{q} \geq \frac{N}{2}$ where the matter system preserves the supersymmetry, $N_{B}=0$ and

$$
N_{\text {eff }}=\frac{N}{2}+|\mathrm{q}|
$$

The effective Lagrangian is of the supersymmetric form (3.3) with (3.5), with $N_{\text {eff }}$ given by (3.39). In other regions where the supersymmetry is spontaneously broken in the matter 

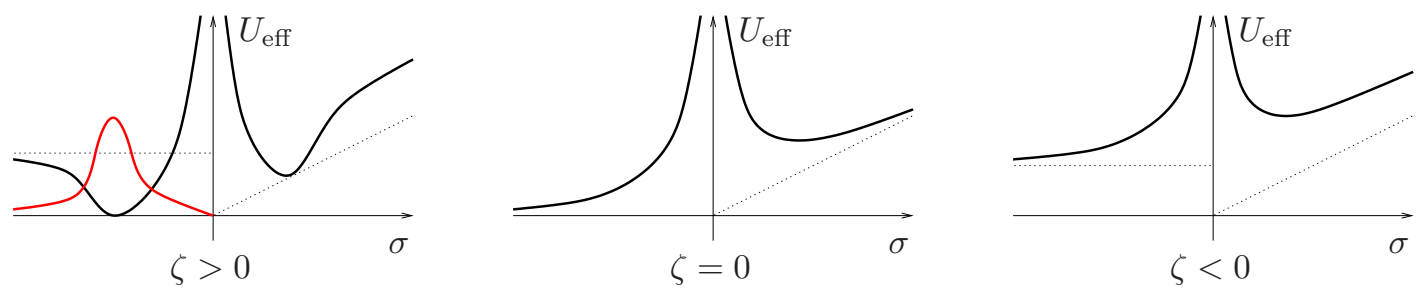

Figure 7. The effective potential for the case $\mathrm{q} \geq \frac{N}{2}$.
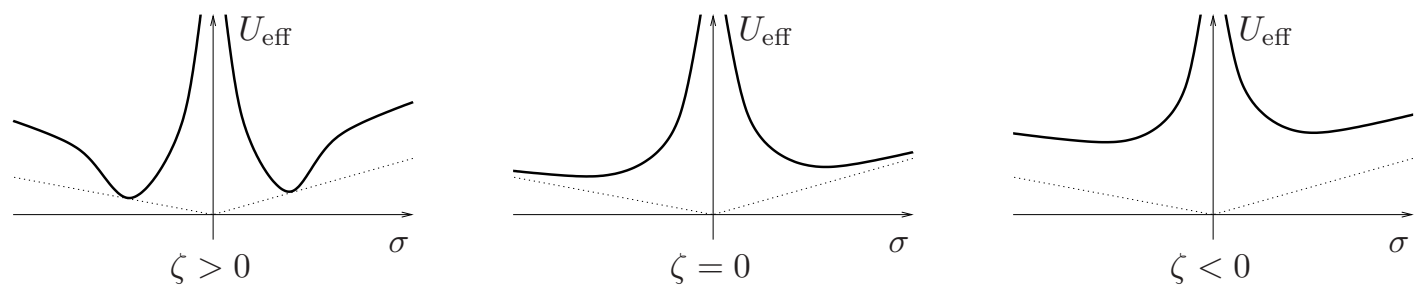

Figure 8. The effective potential for the case $-\frac{N}{2}<\mathrm{q}<\frac{N}{2}$.
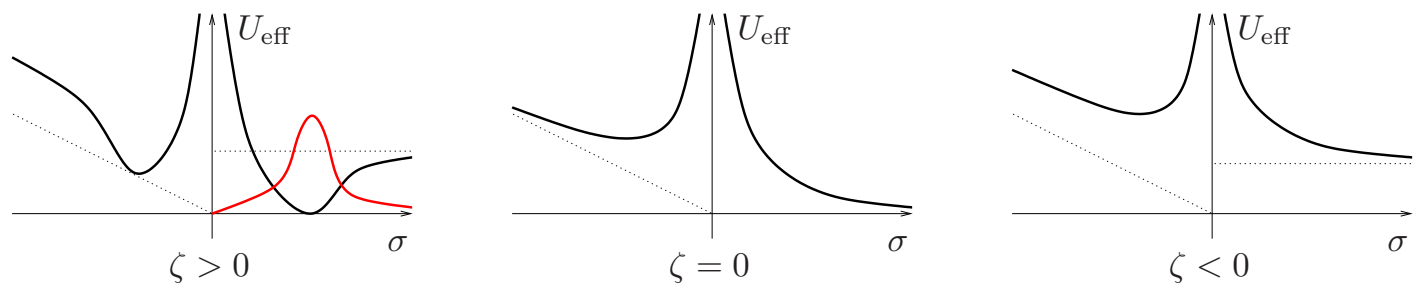

Figure 9. The effective potential for the case $\mathrm{q} \leq-\frac{N}{2}$.

system, $N_{B}$ and $N_{\text {eff }}$ are positive integers which depend on the choice of matter vacuum. We depict the graph of the potential in figure 7,8 and 9 .

The wavefunctions of the supersymmetric ground states, which exist for $\zeta>0$ and $|\mathrm{q}| \geq \frac{N}{2}$, are

$$
\begin{gathered}
\mathrm{q} \geq \frac{N}{2}: \quad \theta(-\sigma)|\sigma|^{\frac{N_{\text {eff }}}{2}} \mathrm{e}^{\zeta \sigma}\left(\prod_{i=1}^{N} \frac{a_{\bar{\phi}_{i}}^{\dagger \bar{n}_{i}}}{\sqrt{\bar{n}_{i} !}}\right)|0\rangle_{\text {osc }}, \quad|\bar{n}|=\mathrm{q}-\frac{N}{2} \\
\mathrm{q} \leq-\frac{N}{2}: \quad \theta(\sigma)|\sigma|^{\frac{N_{\text {eff }}}{2}} \mathrm{e}^{-\zeta \sigma}\left(\prod_{i=1}^{N} \frac{a_{\phi_{i}}^{\dagger n_{i}}}{\sqrt{n_{i} !}}\right) \bar{\psi}_{1} \cdots \bar{\psi}_{N} \bar{\lambda}|0\rangle_{\text {osc }}, \quad|n|=-\mathrm{q}-\frac{N}{2}
\end{gathered}
$$

where $\theta(x)$ is the step function: 1 for $x>0$ and 0 for $x<0$. Note that the R-charge of the latter is bigger than the one of the former by $(N-1)$ : see (2.40). If we assign $r_{\mathrm{c}}=0$ to $\phi$ and take the conjugation symmetric charge assignment to $|0\rangle_{\text {osc }}$, the former and the latter have R-charges $-\frac{N-1}{2}$ and $\frac{N-1}{2}$ respectively. This is the same as the spectrum of the supersymmetric ground states of the theory on the Higgs branch, written in (2.77), (2.78), (2.79). That is, all the supersymmetric ground states deep in the geometric phase $\zeta \gg 0$ become the Coulomb branch ground states for small positive $\zeta$. They disappear to infinity in the limit $\zeta \searrow 0$, and there is no zero energy state in the Coulomb branch for small negative $\zeta$. 
In particular, no supersymmetric ground state is left for $\zeta<0$. This is consistent with the fact that there is no solution to the D-term equation for $\zeta \ll 0$.

For completeness, we describe the Coulomb branch of the $\mathcal{N}=4$ supersymmetric CP ${ }^{N-1}$ model: U(1) gauge group and $N$ chiral multiplets of charge 1 . We see that $N_{\text {eff }}^{(4)}=$ $N$. Therefore, for $\zeta>0$ there are $N$ Coulomb branch vacua constituting the spin $j=\frac{N-1}{2}$ representation of the $\mathrm{SU}(2)$ subgroup of the $\mathrm{U}(2) \subset \mathrm{SO}(4)$ R-symmetry. They run away to infinity as $\zeta \searrow 0$. For $\zeta<0$, there is no supersymmetric vacua.

\subsection{General U(1) theory}

It is straightforward to extend the analysis to a general theory with $\mathrm{U}(1)$ gauge group. Consider the $\mathrm{U}(1)$ gauge theory with chiral multiplets $\left(\phi_{i}, \psi_{i}\right)$ of charge $Q_{i}(i \in \mathrm{I})$, fermi multiplets $\left(\eta_{j}, F_{j}\right)$ of charge $q_{j}(j \in \mathrm{J})$, and Wilson line of charge q, possibly with $E(\phi)$ and/or $J(\phi)$ which are assumed to be quadratic or higher in $\phi$. The anomaly free condition is $\mathrm{q}+\frac{1}{2} \sum_{i} Q_{i}+\frac{1}{2} \sum_{j} q_{j} \in \mathbf{Z}$. We also assume that there is an Abelian flavor symmetry group $T_{F}$ which acts on the fields with the charges $Q_{i}^{f}, q_{j}^{f}$ and have Wilson line of charge $\mathrm{q}^{f}$, where $f \in \mathrm{F}$ is the label of the generators of $T_{F}$.

The matter Hamiltonian and the gauge $\mathrm{U}(1)$ charge are

$$
\begin{aligned}
H_{\text {matter }} & =\sum_{i}\left\{\omega_{i}\left(a_{i}^{\dagger} a_{i}+a_{\bar{\imath}}^{\dagger} a_{\bar{\imath}}+1\right)-Q_{i} \sigma \frac{\left[\bar{\psi}_{i}, \psi_{i}\right]}{2}\right\}+\sum_{j} q_{j} \sigma \frac{\left[\bar{\eta}_{j}, \eta^{j}\right]}{2}, \\
\mathbf{G}_{\text {matter }} & =\sum_{i} Q_{i}\left\{a_{i}^{\dagger} a_{i}-a_{\bar{\imath}}^{\dagger} a_{\bar{\imath}}+\frac{\left[\bar{\psi}_{i}, \psi_{i}\right]}{2}\right\}+\sum_{j} q_{j} \frac{\left[\bar{\eta}_{j}, \eta^{j}\right]}{2}
\end{aligned}
$$

to the quadratic order. Here $\omega_{i}=\sqrt{\left(Q_{i} \sigma\right)^{2}-Q_{i} D}$. The expression for the flavor charge $\mathbf{G}^{f}$ is obtained from (3.43) by the replacement $Q_{i} \rightarrow Q_{i}^{f}$ and $q_{j} \rightarrow q_{j}^{f}$. Higher order terms exists in $H$ if there is an $E(\phi)$ and/or $J(\phi)$. In the present analysis at large $|\sigma|$, however, they have effects suppressed by inverse powers of $|\sigma|$ and will be ignored. We label the states as in (3.25), where we write $n_{i}, \bar{n}_{i}, m_{i}$ and $m_{j}$ for the powers of the creation operators. The energy and the charge of the state are

$$
\begin{aligned}
E_{\text {matter }} & =\sum_{i}\left\{\omega_{i}\left(n_{i}+\bar{n}_{i}+1\right)-Q_{i} \sigma\left(m_{i}-\frac{1}{2}\right)\right\}+\sum_{j} q_{j} \sigma\left(m_{j}-\frac{1}{2}\right), \\
Q_{\text {matter }} & =\sum_{i} Q_{i}\left\{n_{i}-\bar{n}_{i}+m_{i}-\frac{1}{2}\right\}+\sum_{j} q_{j}\left(m_{j}-\frac{1}{2}\right) .
\end{aligned}
$$

The expression for $Q^{f}$ is similar. Under the Gauss law $Q_{\text {matter }}+\mathrm{q}=0$ and in the supersymmetric background $D=0$ where $\omega_{i}=\left|Q_{i} \sigma\right|$, the energy $E=E_{\text {matter }}+q \sigma$ is indeed non-negative,

$$
\begin{aligned}
E & =\sum_{i}\left\{\left|Q_{i} \sigma\right|\left(n_{i}+\bar{n}_{i}+1\right)-Q_{i} \sigma\left(n_{i}-\bar{n}_{i}\right)-Q_{i} \sigma\left(2 m_{i}-1\right)\right\} \\
& =2|\sigma| \times\left\{\begin{array}{l}
\sum_{Q_{i}>0} Q_{i}\left(n_{i}+m_{i}\right)+\sum_{Q_{i}<0}\left|Q_{i}\right|\left(\bar{n}_{i}+\left(1-m_{i}\right)\right) \sigma<0 \\
\sum_{Q_{i}>0} Q_{i}\left(\bar{n}_{i}+\left(1-m_{i}\right)\right)+\sum_{Q_{i}<0}\left|Q_{i}\right|\left(n_{i}+m_{i}\right) \sigma>0 .
\end{array}\right.
\end{aligned}
$$


It vanishes under the following conditions:

$$
\begin{gathered}
\sigma<0: \quad Q_{i}>0 \Rightarrow n_{i}=m_{i}=0 ; \quad Q_{i}<0 \Rightarrow \bar{n}_{i}=1-m_{i}=0, \\
-\sum_{Q_{i}>0} Q_{i}\left(\bar{n}_{i}+\frac{1}{2}\right)+\sum_{Q_{i}<0} Q_{i}\left(n_{i}+\frac{1}{2}\right)+\sum_{j} q_{j}\left(m_{j}-\frac{1}{2}\right)+\mathrm{q}=0, \\
\sigma>0: \quad Q_{i}>0 \Rightarrow \bar{n}_{i}=1-m_{i}=0 ; \quad Q_{i}<0 \Rightarrow n_{i}=m_{i}=0, \\
\sum_{Q_{i}>0} Q_{i}\left(n_{i}+\frac{1}{2}\right)-\sum_{Q_{i}<0} Q_{i}\left(\bar{n}_{i}+\frac{1}{2}\right)+\sum_{j} q_{j}\left(m_{j}-\frac{1}{2}\right)+\mathrm{q}=0 .
\end{gathered}
$$

For each solution to this equation, we have a supersymmetric effective theory on the Coulomb branch with

$$
N_{\mathrm{eff}}=\sum_{Q_{i}>0}\left(n_{i}+\bar{n}_{i}+1\right)-\sum_{Q_{i}<0}\left(n_{i}+\bar{n}_{i}+1\right) .
$$

Suppose $N_{\text {eff }}>0$. Then, the effective theory has a supersymmetric ground state for $\zeta>0$,

$$
\begin{aligned}
& \sigma<0: \quad|\sigma|^{\frac{N_{\mathrm{eff}}}{2}} \mathrm{e}^{\zeta \sigma}\left|n_{\phi}, \bar{n}_{\phi}, m_{\phi}, m_{\eta}\right\rangle, \quad(-1)^{F}=(-1)^{|m|}, \\
& \sigma>0:|\sigma|^{\frac{N_{\mathrm{eff}}}{2}} \mathrm{e}^{-\zeta \sigma} \bar{\lambda}\left|n_{\phi}, \bar{n}_{\phi}, m_{\phi}, m_{\eta}\right\rangle,(-1)^{F}=(-1)^{|m|+1},
\end{aligned}
$$

which runs away to infinity as $\zeta \searrow 0$. For $\zeta<0$, it has no supersymmetric ground state. Suppose $N_{\text {eff }}<0$. Then, the effective theory does not have a supersymmetric ground state for $\zeta>0$. For $\zeta<0$, it has a supersymmetric ground state

$$
\begin{gathered}
\sigma<0:|\sigma|^{\frac{\left|N_{\text {eff }}\right|}{2}} \mathrm{e}^{-\zeta \sigma} \bar{\lambda}\left|n_{\phi}, \bar{n}_{\phi}, m_{\phi}, m_{\eta}\right\rangle,(-1)^{F}=(-1)^{|m|+1} \\
\sigma>0: \quad|\sigma|^{\frac{\left|N_{\text {eff }}\right|}{2}} \mathrm{e}^{\zeta \sigma}\left|n_{\phi}, \bar{n}_{\phi}, m_{\phi}, m_{\eta}\right\rangle, \quad(-1)^{F}=(-1)^{|m|},
\end{gathered}
$$

which runs away to infinity as $\zeta \nearrow 0$. Finally, suppose $N_{\text {eff }}=0$. Then, the effective theory has a zero energy state both for $\zeta>0$ and $\zeta<0$, with the profile as in (3.50)-(3.51) and (3.52)-(3.53). It is square normalizable but it is not clear if it qualifies as the Coulomb branch vacua since its profile is peaked near the origin. However, the wavefunction spreads out to infinity as $\zeta \rightarrow 0$. Hence there is a chance that they play a rôle in the wall crossing phenomenon.

Let us compute the change of the index $I\left(y^{\mathbf{G}^{F}}\right)$, where $y^{\mathbf{G}^{F}}=\prod_{f} y_{f}^{\mathbf{G}^{f}}$, as $\zeta$ goes from positive to negative. There is no subtlety concerning the contribution from the sector with $N_{\text {eff }}>0$ or $N_{\text {eff }}<0$ : the states (3.50) and (3.51) go away to infinity as $\zeta \searrow 0$ or the states (3.52) and (3.53) come in from infinity as $\zeta<0$ is turned on. The contributions to the change in the index in the two cases take the same form, $(-1)^{|m|+1} \prod_{f} y_{f}^{Q^{f}}$ from the $\sigma<0$ branch and $(-1)^{|m|} \prod_{f} y_{f}^{Q^{f}}$ from the $\sigma>0$ branch, where $Q^{f}$ is

$$
\begin{aligned}
& \sigma<0:-\sum_{Q_{i}>0} Q_{i}^{f}\left(\bar{n}_{i}+\frac{1}{2}\right)+\sum_{Q_{i}<0} Q_{i}^{f}\left(n_{i}+\frac{1}{2}\right)+\sum_{j} q_{j}^{f}\left(m_{j}-\frac{1}{2}\right)+\mathrm{q}^{f}, \\
& \sigma>0: \sum_{Q_{i}>0} Q_{i}^{f}\left(n_{i}+\frac{1}{2}\right)-\sum_{Q_{i}<0} Q_{i}^{f}\left(\bar{n}_{i}+\frac{1}{2}\right)+\sum_{j} q_{j}^{f}\left(m_{j}-\frac{1}{2}\right)+\mathrm{q}^{f} .
\end{aligned}
$$


Subtle is what to do for the sector with $N_{\text {eff }}=0$ where the states do not run away but simply spread out as $\zeta \rightarrow 0$. The contribution to the change of the index takes the same form as the $N_{\text {eff }} \neq 0$ cases if we take the average - a "half" of (3.50) and (3.51) go away to infinity and a "half" of (3.52) and (3.53) come in from infinity. If we decide to do so, then, the change of the index takes a concise formula:

$$
\Delta_{C} I\left(y^{\mathbf{G}^{F}}\right)=\sum_{(3.47)}(-1)^{|m|+1} \prod_{f} y_{f}^{Q^{f}}+\sum_{(3.48)}(-1)^{|m|} \prod_{f} y_{f}^{Q^{f}} .
$$

We will see that this is in perfect agreement with the wall crossing formula that will be derived in section 5. This invites us to propose that, for the $N_{\text {eff }}=0$ sector, a "half" of the ground state for $\zeta>0$ plus a "half" of the ground state for $\zeta<0$ contribute to the wall crossing of the index as if they were a single state. Recall, however, that the $N_{\text {eff }}=0$ states have main support toward the origin $\sigma \rightarrow 0$ of the Coulomb branch, and hence we need more information there to say whether the state really exists. The index result is understandable if the state exists only in the $\zeta>0$ side or only in the $\zeta<0$ side.

In the above computation of $\Delta_{C} I\left(y^{\mathrm{G}^{F}}\right)$, we assumed that the twist parameters are unitary $y_{f}=\mathrm{e}^{i \xi_{f}}$, so that we do not need to turn on the real mass $m_{f}$. Turning on real masses would change the form of the Hamiltonian and we would need to start the analysis over. However, the wall crossing part is sensitive only to the behaviour of the wavefunction at large values of $|\sigma|$ to which the finite real masses have only a minor effect. The only effect we need to have in mind is the the deformed supersymmetry algebra (2.47) which dictates how $m_{f}$ enters into the result: that is, we can simply set $y_{f}=\mathrm{e}^{i\left(\xi_{f}+i \beta m_{f}\right)}$ in the result (3.56). This can also be seen buy looking at the result (3.56) itself. It is a Laurent polynomial in $y_{f}$ 's and the holomorphic extension from $y_{f}=\mathrm{e}^{i \xi_{f}}$ to $y_{f}=\mathrm{e}^{i\left(\xi_{f}+i \beta m_{f}\right)}$ is straightforward. This is so even when the theory is not effectively compact. (Of course, in an effectively compact theory the replacement $\mathrm{e}^{i \xi_{f}} \rightarrow \mathrm{e}^{i\left(\xi_{f}+i \beta m_{f}\right)}$ is valid everywhere.) Thus, the wall crossing formula (3.56) is valid for arbitrary complex twist parameters $y_{f} \in \mathbf{C}^{\times}$, whether or not the theory is effectively compact.

Let us also write down the wall crossing formula in $\mathcal{N}=4$ systems with $\mathrm{U}(1)$ gauge group. We recall that the Coulomb branch vacua exists only when $\zeta$ has the same sign as $N_{\text {eff }}^{(4)}$, defined in (3.12). They are invariant under U(1) R-symmetry (so that $\boldsymbol{J}_{-}=-J_{3}$ ) and form a representation of the $\mathrm{SU}(2)$ R-symmetry group of spin $j=\frac{\left|N_{\text {eff }}^{(4)}\right|-1}{2}$, which has character $\chi\left(\mathbf{y}^{-2 J_{3}}\right)=\mathbf{y}^{-2 j}+\mathbf{y}^{-2 j+2}+\cdots+\mathbf{y}^{2 j}$. As a convention, we assign $(-1)^{F}=(-1)^{2 J_{3}}$. Therefore, as $\zeta$ goes from positive to negative, the change of the index is

$$
\Delta_{C} I\left(\mathbf{y}^{2 \boldsymbol{J}_{-}} y^{\mathbf{G}^{F}}\right)=(-1)^{N_{\mathrm{eff}}^{(4)}} \operatorname{sign}\left(N_{\mathrm{eff}}^{(4)}\right)\left(\mathbf{y}^{-\left(\left|N_{\mathrm{eff}}^{(4)}\right|-1\right)}+\cdots+\mathbf{y}^{\left|N_{\mathrm{eff}}^{(4)}\right|-1}\right) .
$$

This is true even when the theory is effectively non-compact and when there is a flavor twist, as included in the notation. This is because the wall crossing states belong to a discrete part of the spectrum in which the effect of the real and twisted masses is continuous. In particular, the operator identity (2.57) makes sense on those states and we can prove that all the Coulomb branch vacua that contribute to the wall crossing have zero flavor charge. 


\subsection{Simple wall crossing}

Let us next consider theories with higher rank gauge groups. On the wall between two phases, there are classical vacua with continuous unbroken gauge symmetries. In general, the symmetry breaking pattern is complex and it is not clear how to analyze the wall crossing. For example, in the III-IV wall in the triangle quiver with rank vector $(k, 1,1)$ with $k \geq 3$ (see figure 4), the space of classical vacua is stratified by mixed branches of different unbroken gauge groups. However, in some cases, we have a simple symmetry breaking where the unbroken gauge group is isomorphic to $\mathrm{U}(1)$. This is always the case in the interior of a wall in a theory with an Abelian gauge group, but there can be such walls even in non-Abelian gauge theories. Let us analyze the wall crossing across such a wall, which we shall refer to as simple.

Thus, suppose we have a simple wall between two phases, with the unbroken gauge group $G_{1} \subset G$ isomorphic to $\mathrm{U}(1)$. When $\zeta$ is close to such a wall region, we must look at the effective theory on the mixed Coulomb-Higgs branch where the $G_{1}$ component of the vector multiplet scalar field is very large while the complementary gauge group $C\left(G_{1}\right) / G_{1}$ is Higgsed. Here $C\left(G_{1}\right) \subset G$ is the subgroup of commutants of $G_{1}$. We decompose the fields into two groups: (C) those which are charged under $G_{1}$ and $(\mathrm{H})$ those which are invariant under $G_{1}$. When $G$ is non-Abelian, the components of the vector multiplet which do not commute with $G_{1}$ should be included in (C) - as fermi multiplets in $\mathcal{N}=2$ theories and as chiral multiplets in $\mathcal{N}=4$ theories. Accordingly we have two theories:

Theory (C) gauge group $G_{1}$, matters from class (C), and the FI parameter close to zero,

Theory (H) gauge group $C\left(G_{1}\right) / G_{1}$, matters from class $(\mathrm{H})$ and the FI parameter in the wall region.

The effective theory on the mixed branch is the "semi-direct product" of the Coulomb branch of Theory $(\mathrm{C})$ and the Higgs branch of Theory $(\mathrm{H})$. We say "semi-direct" because each vacuum of Theory (C) may carry its own charge under $C\left(G_{1}\right) / G_{1}$, which provides a background charge, i.e., the Wilson line, in Theory (H). Note that $C\left(G_{1}\right) / G_{1}$ is regarded as a flavor symmetry in Theory $(\mathrm{C})$.

In $\mathcal{N}=4$ theories, however, Coulomb branch vacua of $(\mathrm{C})$ that contribute to the wall crossing has charge zero under the flavor symmetry, as shown right above. Therefore, the Coulomb and the Higgs dynamics decouples and the mixed branch is the direct product of the Coulomb and the Higgs branches. In particular, supersymmetric ground states are the tensor products of the ground states on the two branches, and the contribution to the change of the index is the simple product:

$$
\Delta I\left(\mathbf{y}^{2 \boldsymbol{J}_{-}} y^{\mathbf{G}^{F}}\right)=I_{\text {wall }}^{(\mathrm{H})}\left(\mathbf{y}^{2 \boldsymbol{J}_{-}} y^{\mathbf{G}^{F}}\right) \times \Delta_{C} I^{(\mathrm{C})}\left(\mathbf{y}^{2 \boldsymbol{J}_{-}}\right) .
$$

Let us illustrate this in the wall crossing in the Abelian triangle quiver (Example $3, \vec{k}=$ $(1,1,1))$. We look at the wall between Phase I and Phase II (see figure 3). The unbroken gauge group is $G_{1}=\{[z, 1,1]\} \cong \mathrm{U}(1)$ and the fields are decomposed as

(C) $y_{1, \ldots, b}(1), z_{1, \ldots, c}(-1)$,

(H) $x_{1, \ldots, a}(1)$. 
To the right of the fields, we put their charges with respect to $G_{1}$ for (C) and $G / G_{1}$ for $(\mathrm{H})$. The Coulomb branch of $(\mathrm{C})$ has $N_{\mathrm{eff}}^{(4)}=b-c$ so that we have $\Delta_{C} I^{(\mathrm{C})}\left(\mathrm{y}^{2 J_{-}}\right)=$ $(-1)^{b-c-1} \operatorname{sign}(c-b)\left(\mathbf{y}^{-(|b-c|-1)}+\cdots+\mathbf{y}^{|b-c|-1}\right)$. The Higgs branch of $(\mathrm{H})$ is $\mathbf{C P}^{a-1}$ which has $I^{(\mathrm{H})}\left(\mathbf{y}^{2 \boldsymbol{J}_{-}}\right)=(-1)^{a-1}\left(\mathbf{y}^{-(a-1)}+\cdots+\mathbf{y}^{a-1}\right)$. Thus, as $\zeta$ moves from Phase $\mathrm{I}$ to Phase II, the index changes as

$$
\Delta I\left(\mathbf{y}^{2 \boldsymbol{J}_{-}}\right)=(-1)^{a+b+c}\left(\mathbf{y}^{-(a-1)}+\cdots+\mathbf{y}^{a-1}\right) \cdot \operatorname{sign}(c-b)\left(\mathbf{y}^{-(|b-c|-1)}+\cdots+\mathbf{y}^{|b-c|-1}\right) .
$$

We will see more examples in later sections.

In a theory without $\mathcal{N}=4$ supersymmetry, each vacuum may have a non-trivial flavor charge and hence Theory $(\mathrm{H})$ may have different background charges for different $(\mathrm{C})$ vacua. Therefore, the change in the index is not in general the simple product, but is a "semi-direct" product, which may be denoted as

$$
\Delta I\left(y^{\mathrm{G}^{F}}\right)=\left(I_{\text {wall }}^{(\mathrm{H})} * \Delta_{C} I^{(\mathrm{C})}\right)\left(y^{\mathrm{G}^{F}}\right)
$$

We will present illustrative examples in later sections.

\section{The index}

In this section, we compute the Witten index

$$
I\left(y^{\mathbf{G}^{F}}\right)=\operatorname{Tr}_{\mathcal{H}}\left((-1)^{F} y^{\mathbf{G}^{F}} \mathrm{e}^{-\beta H}\right) .
$$

As we have seen, the spectrum of supersymmetric ground states depends very much on the region where the FI parameter $\zeta$ belongs. When it is deep inside a phase where the gauge group is broken to a finite group, the ground state spectrum is that of the effective theory on the Higgs branch, and in particular the index should agree with the Higgs branch index. "Deep inside" means that $1 / \beta$ is comparable to or smaller than the Higgs mass $M_{H}=e \sqrt{|\Delta \zeta|}($ see $(2.62))$,

$$
e^{2}|\Delta \zeta| \sim \frac{1}{\beta^{2}}
$$

where $|\Delta \zeta|$ is the distance to the walls of the phase. As $\zeta$ approaches a wall, some of the ground states start to have supports on the Coulomb branch and eventually disappear by running away to infinity. As a consequence, the index must undergo a transition as $\zeta$ goes across the wall. The effective theory on the Coulomb branch has a characteristic energy scale $M_{C}=e^{2}|\Delta \zeta|^{2}$ (see (3.9)). Thus, the wall crossing transition is expected within a range where it is vanishingly small compared to $1 / \beta$,

$$
e^{2}|\Delta \zeta|^{2} \ll \frac{1}{\beta}
$$

We shall employ the method of supersymmetric localization to compute the index, which includes taking the limit $e^{2} \rightarrow 0$. This limit is potentially singular because it turns off the D-term potential and the Higgs mass. The singularity is especially severe in the one-dimensional system because of the non-compact flat direction in the Coulomb branch. 
As we will see, in order to obtain a sensible answer, we need to take particular scaling limits in which $\Delta \zeta$ is sent to infinity. In addition, there is a subtlety in the definition of the index associated to the non-compactness, and some of the above expectation needs to be reexamined. All these are unlike the similar computation of the elliptic genus of two-dimensional $(2,2)$ supersymmetric theories $[39,40]$ where the Coulomb branch is lifted by the R-symmetry twist.

\subsection{Setting up the computation}

We consider a general $\mathcal{N}=2$ supersymmetric gauge theory introduced in section 2.1, with the gauge group $G$, the matter contents specified by $V_{\text {chiral }}, V_{\text {fermi }}$ and $M$, and the interaction given by $\zeta, E(\phi), J(\phi)$ and $Q(\phi)$. We suppose that there is a group $G_{F}$ of flavor symmetries.

The index (4.1) is equal to the path integral on the Euclidean circle of circumference $\beta$ with periodic boundary condition, with the flavor Wilson line $v_{\tau}^{F}$ and real mass $m$ turned on, for $y=\mathrm{e}^{-\beta\left(i v_{\tau}^{F}+m\right)}$,

$$
I=\int \mathcal{D} \mu \exp \left(-\int_{0}^{\beta} L_{E} \mathrm{~d} \tau\right) \operatorname{Str}_{M} \operatorname{Pexp}\left(-\int_{0}^{\beta} i \mathcal{A}_{\tau} \mathrm{d} \tau\right) .
$$

The Euclidean Lagrangian is obtained by the Wick rotation $t \rightarrow-i \tau$, which includes the rotation of the auxiliary fields, $D \rightarrow i D_{E}$, where $D_{E}$ is real valued (i.e. $D_{E} \in i \mathfrak{g}$ ), and $F \rightarrow i F_{E}, \bar{F} \rightarrow i F_{E}^{\dagger}:$

$$
\begin{aligned}
L_{E}= & \frac{1}{2 e^{2}} \operatorname{Tr}\left[\left(D_{\tau} \sigma\right)^{2}+\bar{\lambda} D_{\tau}^{(+)} \lambda+D_{E}^{2}\right]+i \zeta\left(D_{E}\right) \\
& +\frac{1}{\mathrm{~g}^{2}}\left[\tilde{D}_{\tau} \bar{\phi} \tilde{D}_{\tau} \phi+\bar{\psi} \tilde{D}_{t}^{(-)} \psi+\bar{\phi}\left\{-i D_{E}+\tilde{\sigma}^{2}\right\} \phi+i \bar{\phi} \lambda \psi-i \bar{\psi} \bar{\lambda} \phi\right] \\
& +\frac{1}{\mathrm{~g}^{2}}\left[\bar{\eta} \tilde{D}_{\tau}^{(+)} \eta+F_{E}^{\dagger} F_{E}+\overline{E(\phi)} E(\phi)+\bar{\eta} \partial_{i} E(\phi) \psi^{i}+\bar{\psi}^{\bar{\imath}} \partial_{\bar{\imath}} \overline{E(\phi)} \eta\right] \\
& -\psi^{i} \partial_{i} J(\phi) \eta+i J(\phi) F_{F}-\bar{\eta} \partial_{\bar{\imath}} J(\phi)^{\dagger} \bar{\psi}^{\bar{\imath}}+i F_{E}^{\dagger} J(\phi)^{\dagger}, \\
i \mathcal{A}_{\tau}= & \rho\left(i \tilde{v}_{\tau}+\tilde{\sigma}\right)-\psi^{i} \partial_{i} Q(\phi)+\bar{\psi}^{\bar{\tau}} \partial_{\bar{\imath}} Q(\phi)^{\dagger}+\left\{Q(\phi), Q(\phi)^{\dagger}\right\} .
\end{aligned}
$$

Here we put $\tilde{v}_{\tau}:=v_{\tau}+v_{\tau}^{F}$ and $\tilde{\sigma}:=\sigma+m$, and in particular, we have $\tilde{D}_{\tau}^{( \pm)}=\frac{\mathrm{d}}{\mathrm{d} \tau}+i \tilde{v}_{\tau} \pm \tilde{\sigma}$ We assume that there is a region of parameters $\zeta, v_{\tau}^{F}, m, J(\phi)$, etc, where the theory is effectively compact. Then, the index is well-defined and is independent of parameters as long as they are in that region (except the dependence on $i v_{\tau}^{F}+m$ due to $(2.47)$ ). In particular, it is independent of $e$ and $\mathrm{g}$, and we evaluate the path-integral in the regime where $e$ and $g$ are very small. The basic strategy to evaluate the path-integral is mostly the same as in the computation $[39,40]$ of $2 \mathrm{~d}$ index, but we shall see important differences.

We will eventually take the limit where the gauge coupling constant $e$ is sent to zero. In that limit, the path-integral is dominated by the configurations satisfying

$$
D_{\tau} \sigma=0, \quad D_{E}=0
$$


The only observable of a gauge field on the circle is its holonomy, $h=\operatorname{Pexp}\left(-i \int_{0}^{\beta} v_{\tau} \mathrm{d} \tau\right)$, and a parallel section $\sigma$ is determined by its value at $\tau=0$ that commutes with $h$. Therefore, the moduli space $\mathfrak{M}$ of solutions to (4.7) is the space of commuting pairs in $G \times i \mathfrak{g}$ modulo the adjoint action, which is isomorphic to

$$
\mathfrak{M}=(T \times i \mathfrak{t}) / W
$$

where $T$ is a maximal torus of $G$ and $W$ is the Weyl group of $(G, T)$. A solution is realized by constant profiles with values in $i \mathrm{t}, v_{\tau} \equiv v_{\tau 0}^{\mathrm{t}}$ and $\sigma \equiv \sigma_{0}^{\mathrm{t}}$. Since the holonomy is $h=\mathrm{e}^{-i \beta v_{\tau 0}^{t}}$ we see that the shift of $\beta v_{\tau 0}^{\mathrm{t}}$ by an element of $2 \pi \mathrm{Q}^{\vee}$ is a gauge transformation, where $\mathrm{Q}^{\vee} \subset i \mathfrak{t}$ is the coroot lattice - the kernel of the map $\xi \in i \mathfrak{t} \mapsto \mathrm{e}^{2 \pi i \xi} \in T$. It is convenient to take the supersymmetric combination

$$
u:=\frac{\beta}{2 \pi}\left(-v_{\tau 0}^{\mathfrak{t}}+i \sigma_{0}^{\mathfrak{t}}\right) \in \mathfrak{t}_{\mathbf{C}}
$$

which has periodicity $\mathrm{Q}^{\vee}$. It defines a system of complex flat coordinates of the cover $\tilde{\mathfrak{M}}=\mathfrak{t}_{\mathbf{C}} / \mathrm{Q}^{\vee}$ of the moduli space, $\mathfrak{M} \cong \mathfrak{t}_{\mathbf{C}} /\left(\mathrm{Q}^{\vee} \rtimes W\right)=\tilde{\mathfrak{M}} / W$. In contrast to the $2 \mathrm{~d}$ index computation, the moduli space $\mathfrak{M}$ is non-compact, and that is responsible for the wall crossing phenomenon which is the distinguished feature in $1 \mathrm{~d}$.

Note that (4.7) is the condition of supersymmetry, $\delta \lambda=\delta \bar{\lambda}=0$. Therefore, $\mathfrak{M}$ is also the moduli space of supersymmetric background for the vector multiplet. In fact, the above discussion guarantees that our discussion in section 2.2 on the flavor twist and real mass was general enough. Just like (4.9), we write

$$
z:=\frac{\beta}{2 \pi}\left(-v_{\tau}^{F}+i m\right) \in \mathfrak{t}_{F \mathbf{C}}
$$

for the complex parameter of the flavor twist, $y=\mathrm{e}^{2 \pi i z} \in T_{F \mathbf{C}}$.

The index can be written as the integration on the moduli space,

$$
I=\int_{\mathfrak{M}} \mathrm{d}^{2 \ell} u F_{e, \mathrm{~g}}(u)=\frac{1}{|W|} \int_{\tilde{\mathfrak{M}}} \mathrm{d}^{2 \ell} u F_{e, \mathrm{~g}}(u),
$$

where $F_{e, g}(u)$ is the result of path integral over all fields except the commuting zero modes of $v_{\tau}$ and $\sigma$. The integrand $F_{e, g}(u)$ depends on $e$ and g, even though the the integral (4.11) does not. The matter integral for a given gauge multiplet is simplified if we can take the limit $\mathrm{g} \rightarrow 0$. This is certainly valid when the scalar component $\phi$ of the chiral multiplet does not have a zero mode. Along the supersymmetric background specified by $u \in \mathfrak{t}_{\mathbf{C}}$, this is the case except when $\mathrm{e}^{2 \pi i(u+z)}: V_{\text {chiral }} \rightarrow V_{\text {chiral }}$ has eigenvalue 1 . Let

$$
V_{\text {chiral }}=\bigoplus_{i \in \mathrm{I}} \mathbf{C}\left(Q_{i}, Q_{i}^{F}\right)
$$

be the weight decomposition. Then, the $i$-th component $\phi_{i}$ of $\phi$ has a zero mode when

$$
Q_{i}(u)+Q_{i}^{F}(z) \equiv 0 \quad \text { modulo } \mathbf{Z}
$$


We denote the locus (4.13) by $H_{i} \subset \tilde{\mathfrak{M}}$ and call it a singular hyperplane for the field $\phi_{i}$. If $u$ is away from any of such hyperplanes, then, the limit $\mathrm{g} \rightarrow 0$ can obviously be taken. We further make the following assumption that allows $\mathrm{g} \rightarrow 0$ (for non-zero $e$ ) even along singular hyperplanes: at each point $u_{*} \in \tilde{\mathfrak{M}}$, let $\left(\phi_{i}\right)_{i \in \mathrm{I}_{u_{*}}}$ be the set of fields which has zero modes at $u_{*}$. Then, we assume that the space of $\left(\phi_{i}\right)_{i \in \mathrm{I}_{u_{*}}}$ solving the D-term equations is compact. For example, for $G=\mathrm{U}(1)$, this means that the fields having zero modes at each point of $\tilde{\mathfrak{M}}=\mathfrak{M}$ have either positive charges or negative charges, but not both. Under this assumption, the limit $\mathrm{g} \rightarrow 0$ can be taken everywhere on $\tilde{\mathfrak{M}}$, provided $e$ is non-zero. Moreover, this allows us to ignore the contribution from an infinitesimal neighborhood of the singular hyperplanes and take a limit $e \rightarrow 0$ at the same time. To state it precisely, let us put

$$
\Delta_{\varepsilon}:=\bigcup_{i \in \mathrm{I}} \Delta_{\varepsilon}\left(H_{i}\right)
$$

where $\Delta_{\varepsilon}\left(H_{i}\right)$ is the $\varepsilon$-neighborhood of $H_{i}$ in $\tilde{\mathfrak{M}}$. Then, there is a double scaling limit $e \rightarrow 0$ and $\varepsilon \rightarrow 0$ under which the contribution from $\Delta_{\varepsilon}$ at $\mathrm{g}=0$ vanishes, so that the integral (4.11) can be replaced by

$$
I=\frac{1}{|W|} \lim _{\substack{e \rightarrow 0 \\ \varepsilon \rightarrow 0}} \int_{\tilde{\mathfrak{M}} \backslash \Delta_{\varepsilon}} \mathrm{d}^{2 \ell} u F_{e, 0}(u)
$$

Thus, we would like to compute $F_{e, \mathrm{~g}}(u)$ for $u$ away from the singular hyperplanes, in the limit $e \rightarrow 0$ and $\mathrm{g} \rightarrow 0$. Note that the moduli variable $u$, i.e., $v_{\tau 0}^{\mathrm{t}}$ and $\sigma_{0}^{\mathrm{t}}$ are not closed under the supersymmetry (2.4) but is a part of the supermultiplet consisting of the constant modes of the Cartan part of the vector multiplet $\left(v_{\tau 0}^{\mathfrak{t}}, \sigma_{0}^{\mathfrak{t}}, \lambda_{0}^{\mathfrak{t}}, \bar{\lambda}_{0}^{\mathfrak{t}}, D_{E 0}^{\mathfrak{t}}\right)$. To obtain $F_{e, \mathrm{~g}}(u)$, we first do the path-integral over all the supermultiplets which are orthogonal to this Cartan zero modes, and then do the integration over $\lambda_{0}^{\mathfrak{t}}, \bar{\lambda}_{0}^{\mathrm{t}}$ and $D_{E 0}^{\mathrm{t}}$. In the limit $e \rightarrow 0$ and $\mathrm{g} \rightarrow 0$, the first part can be done exactly by the one-loop integral.

\subsection{One loop integral}

Vector multiplet. We write the vector multiplet modes which are orthogonal to the Cartan zero modes as $\left(v_{\tau}^{\prime}, \sigma^{\prime}, \lambda^{\prime}, \bar{\lambda}^{\prime}, D_{E}^{\prime}\right)$. The gauge fixing amounts to dropping the integral over $\left(v_{\tau}^{\prime}, \sigma^{\prime}\right)$. The simplest way to see this is to note that the Gauss law is simply $D_{\tau} \sigma=0$ in the limit $e \rightarrow 0$, which sets $v_{\tau}^{\prime}=\sigma^{\prime}=0$ and leaves us with the integral over the moduli space $\mathfrak{M}$. Thus, the one-loop integral is just over $\left(\lambda^{\prime}, \bar{\lambda}^{\prime}, D_{E}^{\prime}\right)$, which yields

$$
Z_{\text {vector }}^{\prime}=\operatorname{det}^{\prime} D_{\tau 0}^{\mathfrak{t}(+)}
$$

The operator $D_{\tau 0}^{\mathfrak{t}(+)}=\frac{\mathrm{d}}{\mathrm{d} \tau}+\operatorname{ad}\left(i v_{\tau 0}^{\mathfrak{t}}+\sigma_{0}^{\mathfrak{t}}\right)=\frac{\mathrm{d}}{\mathrm{d} \tau}-\frac{2 \pi i}{\beta} \operatorname{ad}(u)$ is diagonalized via the Fourier modes $\mathrm{e}^{-2 \pi i \mathrm{~m} \tau / \beta}$ and the root decomposition of $\mathfrak{g}_{\mathbf{C}}$, and we find (we omit writing $( \pm i) \frac{2 \pi}{\beta}$ that multiplies each eigenvalue)

$$
Z_{\text {vector }}^{\prime}=\left(\prod_{\mathrm{m} \neq 0} \mathrm{~m}\right)^{\ell} \cdot \prod_{\alpha} \prod_{\mathrm{m} \in \mathbf{Z}}(\mathrm{m}+\alpha(u))
$$




$$
\propto \prod_{\alpha}\left(\mathrm{e}^{-\pi i \alpha(u)}-\mathrm{e}^{\pi i \alpha(u)}\right)
$$

Each root factor in (4.17) can also be understood from the operator formalism: the index of the $\lambda_{\alpha}, \bar{\lambda}_{\alpha}$ system may be written as $Z_{\alpha}=\operatorname{Tr}(-1)^{F} \mathrm{e}^{2 \pi i \mathbf{G}(u)} \mathrm{e}^{-\beta H}$, with $H=0$ and $\mathbf{G}(u)=\frac{\alpha(u)}{2}\left[\bar{\lambda}_{\alpha}, \lambda_{\alpha}\right]$. If we assign $(-1)^{F}=1$ to the Fock vacuum annihilated by $\lambda_{\alpha}$, then we find $Z_{\alpha}=\mathrm{e}^{-\pi i \alpha(u)}-\mathrm{e}^{\pi i \alpha(u)}$. It may be more natural to put $v_{\tau 0}^{\mathfrak{t}}$ to $\mathbf{G}$ and $\sigma_{0}^{\mathrm{t}}$ to $H$, but the result is the same.

In the remaining part of the one-loop integral, the fluctuation modes $\left(v_{\tau}^{\prime}, \sigma^{\prime}, \lambda^{\prime}, \bar{\lambda}^{\prime}, D_{E}^{\prime}\right)$ will never appear. To simplify the notation, we denote the Cartan zero modes simply as $\left(v_{\tau}, \sigma, \lambda, \bar{\lambda}, D_{E}\right)$, dropping the super/subscripts $\mathfrak{t} / 0$. We also write

$$
D:=\frac{\beta^{2}}{(2 \pi)^{2}} D_{E}
$$

for a dimensionless variable.

Chiral multiplet. Since we shall eventually perform the integral over the $2 \ell$ gluino zero modes, we may drop the terms $i \bar{\phi} \lambda \psi-i \bar{\psi} \bar{\lambda} \phi$ from the exponential, and look only at

$$
\begin{aligned}
Z_{\text {chiral }} & =\int \mathcal{D} \phi \mathcal{D} \psi \mathrm{e}^{-\int_{0}^{\beta} \mathrm{d} \tau\left[\bar{\phi}\left(-\tilde{D}_{\tau}^{2}+\tilde{\sigma}^{2}-i D_{E}\right) \phi+\bar{\psi} \tilde{D}_{\tau}^{(-)} \psi\right]} \frac{1}{(\ell !)^{2}}\left(\int_{0}^{\beta} \bar{\phi} \lambda \psi \mathrm{d} \tau \int_{0}^{\beta} \bar{\psi} \bar{\lambda} \phi \mathrm{d} \tau^{\prime}\right)^{\ell} \\
& =\frac{\operatorname{det} \tilde{D}_{\tau}^{(-)}}{\operatorname{det}\left(-\tilde{D}_{\tau}^{2}+\tilde{\sigma}^{2}-i D_{E}\right)}\left\langle\frac{1}{(\ell !)^{2}}\left(\int_{0}^{\beta} \bar{\phi} \lambda \psi \mathrm{d} \tau \int_{0}^{\beta} \bar{\psi} \bar{\lambda} \phi \mathrm{d} \tau^{\prime}\right)^{\ell}\right\rangle
\end{aligned}
$$

In view of the weight decomposition (4.12), this is equal to

$$
Z_{\text {chiral }}=g_{\text {chiral }}(u, D) \cdot \operatorname{det} h(u, D) \prod_{a=1}^{\ell} \lambda_{a} \bar{\lambda}_{a}
$$

where

$$
g_{\text {chiral }}(u, D):=\frac{\prod_{i \in \mathrm{I}} \prod_{\mathrm{m} \in \mathbf{Z}}\left(\mathrm{m}+Q_{i}(\bar{u})+Q_{i}^{F}(\bar{z})\right)}{\prod_{i \in \mathrm{I}} \prod_{\mathrm{m} \in \mathbf{Z}}\left(\left|\mathrm{m}+Q_{i}(u)+Q_{i}^{F}(z)\right|^{2}-i Q_{i}(D)\right)},
$$

and $\operatorname{det} h(u, D)$ is the determinant of the $\ell \times \ell$ matrix

$$
h^{a b}(u, D):=\sum_{i \in \mathrm{I}} \sum_{\mathrm{m}} \frac{Q_{i}^{a} Q_{i}^{b}}{\left(\left|\mathrm{~m}+Q_{i}(u)+Q_{i}^{F}(z)\right|^{2}-i Q_{i}(D)\right)\left(\mathrm{m}+Q_{i}(\bar{u})+Q_{i}^{F}(\bar{z})\right)} .
$$

If we set $D=0$, the function $g_{\text {chiral }}$ simplifies

$$
\begin{aligned}
g_{\text {chiral }}(u, 0) & =\frac{1}{\prod_{i \in \mathrm{I}} \prod_{\mathrm{m} \in \mathbf{Z}}\left(\mathrm{m}+Q_{i}(u)+Q_{i}^{F}(z)\right)} \\
& \propto \frac{1}{\prod_{i \in \mathrm{I}}\left(\mathrm{e}^{\pi i\left(Q_{i}(u)+Q_{i}^{F}(z)\right)}-\mathrm{e}^{-\pi i\left(Q_{i}(u)+Q_{i}^{F}(z)\right)}\right)} .
\end{aligned}
$$


It is the index of a chiral multiplet in the supersymmetric background, which explains the holomorphic dependence on $u$ and $z$, see section 2.2. The precise form (4.23) can also be understood from the operator formalism. For this purpose, it is enough to consider the $\mathrm{U}(1)$ gauge theory with a single chiral multiplet of charge 1. Quantization of the chiral multiplet in the constant background $\sigma$ has been done in section 3.2, as its special case $N=1$. The energy and the charge spectrum is given in (3.26) and (3.27). If we assign $(-1)^{F}=1$ to the oscillator vacuum $|0\rangle_{\text {osC }}$, we see that the twisted index is

$$
\begin{aligned}
\operatorname{Tr}(-1)^{F} \mathrm{e}^{-i \beta \mathbf{G}\left(v_{\tau}\right)} \mathrm{e}^{-\beta H} & =\sum_{n, \bar{n} \in \mathbf{Z}} \sum_{m=0,1}(-1)^{m} \mathrm{e}^{-i \beta v_{\tau}\left(n-\bar{n}+m-\frac{1}{2}\right)} \mathrm{e}^{-\beta|\sigma|(n+\bar{n}+1)+\beta \sigma\left(m-\frac{1}{2}\right)} \\
& =\mathrm{e}^{-\beta|\sigma|} \frac{\mathrm{e}^{-i \beta v_{\tau}\left(-\frac{1}{2}\right)} \mathrm{e}^{\beta \sigma\left(-\frac{1}{2}\right)}-\mathrm{e}^{-i \beta v_{\tau}\left(\frac{1}{2}\right)} \mathrm{e}^{\beta \sigma\left(\frac{1}{2}\right)}}{\left(1-\mathrm{e}^{-i \beta v_{\tau}} \mathrm{e}^{-\beta|\sigma|}\right)\left(1-\mathrm{e}^{i \beta v_{\tau}} \mathrm{e}^{-\beta|\sigma|}\right)} \\
& \left.=\frac{1}{\mathrm{e}^{\pi i u}-\mathrm{e}^{-\pi i u}} \quad \text { (for both signs of } \sigma\right)
\end{aligned}
$$

which is indeed of the form (4.23).

Fermi multiplet. Let

$$
V_{\mathrm{fermi}}=\bigoplus_{j \in \mathrm{J}} \mathbf{C}\left(q_{j}, q_{j}^{F}\right)
$$

be the weight decomposition. Then, the one loop integral is given by

$$
\begin{aligned}
Z_{\mathrm{fermi}} & =\operatorname{det} D_{\tau}^{(+)}=\prod_{j \in \mathrm{J}} \prod_{\mathrm{m} \in \mathbf{Z}}\left(\mathrm{m}+q_{j}(u)+q_{j}^{F}(z)\right) \\
& \propto \prod_{j \in \mathrm{J}}\left(\mathrm{e}^{-\pi i\left(q_{j}(u)+q_{j}^{F}(z)\right)}-\mathrm{e}^{\pi i\left(q_{j}(u)+q_{j}^{F}(z)\right)}\right) .
\end{aligned}
$$

The final expression can also be understood from the operator formalism.

Wilson line. Let

$$
M=\bigoplus_{k \in \mathrm{K}} \mathbf{C}\left(\mathrm{q}_{k}, \mathrm{q}_{k}^{F}\right)\left[r_{k}\right]
$$

be the weight decomposition, where $r_{k}=0$ for the even part and $r_{k}=1$ for the odd part. Then, the Wilson line gives the following factor

$$
Z_{\text {Wilson }}=\sum_{k \in \mathrm{K}}(-1)^{r_{k}} \mathrm{e}^{2 \pi i\left(\mathrm{q}_{k}(u)+\mathrm{q}_{k}^{F}(z)\right)} .
$$

Summary. After performing the gluino integral, we have

$$
I=\frac{N_{\ell}}{|W|} \lim _{\substack{e \rightarrow 0 \\ \varepsilon \rightarrow 0}} \int_{\tilde{\mathfrak{M}} \backslash \Delta_{\varepsilon}} \mathrm{d}^{2 \ell} u \int_{i \mathrm{t}} \mathrm{d}^{\ell} D g(u, D) \operatorname{det} h(u, D) \exp \left(-\frac{(2 \pi)^{4}}{2 e^{2} \beta^{3}} D^{2}-i \frac{(2 \pi)^{2}}{\beta} \zeta(D)\right),
$$

where $N_{\ell}$ is a normalization constant to be determined, and $g(u, D)$ is the product of $Z_{\text {vector }}^{\prime}(u), g_{\text {chiral }}(u, D), Z_{\text {fermi }}(u)$ and $Z_{\text {Wilson }}(u)$, which is normalized so that $g(u, D=0)$ 
is the product of the right hand sides of (4.17), (4.23), (4.26) and (4.28),

$$
g(u, 0)=\prod_{\alpha} 2 i \sin (-\pi \alpha(u)) \frac{\prod_{j \in \mathrm{J}} 2 i \sin \left(-\pi\left(q_{i}(u)+q_{i}^{F}(z)\right)\right)}{\prod_{i \in \mathrm{I}} 2 i \sin \left(\pi\left(Q_{i}(u)+Q_{i}^{F}(z)\right)\right)} \sum_{k \in \mathrm{K}}(-1)^{r_{k}} \mathrm{e}^{2 \pi i\left(\mathrm{q}_{k}(u)+\mathrm{q}_{k}^{F}(z)\right)} .
$$

It remains to perform the $u$ and $D$ integrals and then take the limit. For this purpose, there is a very useful identity

$$
\frac{\partial}{\partial \bar{u}_{a}} g(u, D)=-i h^{a b}(u, D) D_{b} g(u, D) .
$$

As in $[39,40]$, this can be used to turn the integral into the residue integral around intersections of the singular hyperplanes. We first consider a theory with U(1) gauge group.

\subsection{U(1) theories}

If the gauge group is $\mathrm{U}(1)$, the moduli space is one dimensional, $\tilde{\mathfrak{M}}=\mathfrak{M}=\mathbf{C} / \mathbf{Z} \cong \mathbf{C}^{\times}$, and singular hyperplanes are points of $\mathfrak{M}$. We denote by $\mathfrak{M}_{\text {sing }}^{(+)}$and $\mathfrak{M}_{\text {sing }}^{(-)}$the sets of singular points corresponding to positively charges fields and negatively charged fields respectively. By the assumption made above, they have no overlap, $\mathfrak{M}_{\text {sing }}^{(+)} \cap \mathfrak{M}_{\text {sing }}^{(-)}=\emptyset$. We denote by $\Delta_{\varepsilon}^{( \pm)}$the epsilon neighborhood of $\mathfrak{M}_{\text {sing }}^{( \pm)}$, so that $\Delta_{\varepsilon}=\Delta_{\varepsilon}^{(+)} \sqcup \Delta_{\varepsilon}^{(-)}$. We set $\beta=2 \pi$ to simplify the notation.

For a U(1) theory, the integral (4.29) is

$$
I=N_{1} \lim _{\substack{e \rightarrow 0 \\ \varepsilon \rightarrow 0}} \int_{\mathfrak{M} \backslash \Delta_{\varepsilon}} \mathrm{d}^{2} u \int_{\mathbf{R}} \mathrm{d} D g(u, D) h(u, D) \exp \left(-\frac{\pi}{e^{2}} D^{2}-2 \pi i \zeta D\right),
$$

and the identity (4.31) reads

$$
g(u, D) h(u, D)=\frac{i}{D} \frac{\partial}{\partial \bar{u}} g(u, D)
$$

The plan is to insert (4.33) into (4.32), apply the Stokes theorem in the $u$-integral, and then evaluate the integral at each boundary component. It turns out to be useful to deform the domain of $D$ integral in advance, from the real line $\mathbf{R}$ to a contour in the complex $D$-plane that goes around $D=0$. This is valid as long as the deformation does not hit the poles of the integrand of (4.32). Denoting the new contour by $\Gamma$ and applying the Stokes theorem, we have

$$
I=\frac{N_{1}}{2} \lim _{\substack{e \rightarrow 0 \\ \varepsilon \rightarrow 0}} \int_{\Gamma} \mathrm{d} D \oint_{\partial\left(\mathfrak{M} \backslash \Delta_{\varepsilon}\right)} \mathrm{d} u \frac{1}{D} g(u, D) \exp \left(-\frac{\pi}{e^{2}} D^{2}-2 \pi i \zeta D\right) .
$$

There is a simple pole at $D=0$ in the integrand, even though it must go away after the $u$-integration, as $D=0$ was perfectly regular in (4.32). It goes away if the sum over all components of $\partial\left(\mathfrak{M} \backslash \Delta_{\varepsilon}\right)$ is taken, but survives in the individual component. That is why we deformed the $D$ contour in advance. We denote by $\Gamma_{-}\left(\right.$resp.$\left.\Gamma_{+}\right)$the contour that goes below (resp. above) $D=0$. To be specific, let us take $\Gamma=\Gamma_{-}$. We emphasize that the 

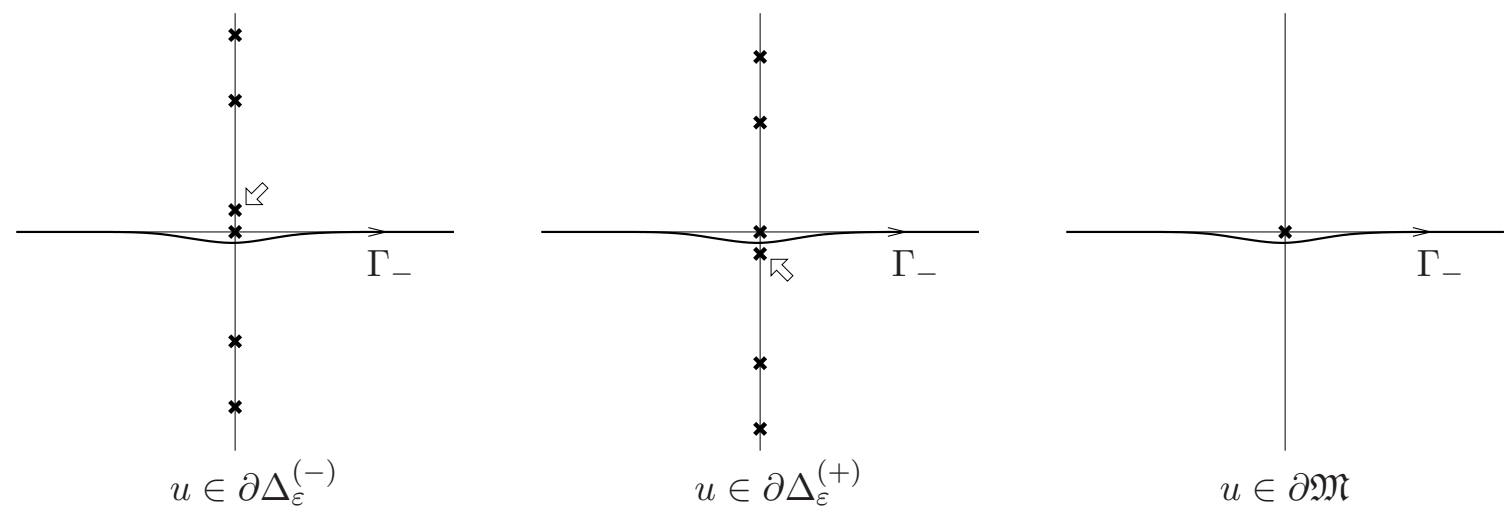

Figure 10. Poles and the contour $\Gamma_{-}$in the complex $D$ plane.

contour deformation should be small enough so as not to hit the poles of (4.32), i.e., those of $g(u, D)$.

Note that $\partial\left(\mathfrak{M} \backslash \Delta_{\varepsilon}\right)$ has three groups of components,

$$
\partial\left(\mathfrak{M} \backslash \Delta_{\varepsilon}\right)=\partial \mathfrak{M}-\partial \Delta_{\varepsilon}^{(+)}-\partial \Delta_{\varepsilon}^{(-)},
$$

where $\partial \mathfrak{M}$ is the boundary at infinity (two circles at $u \rightarrow \pm i \infty$ ). Let us look at a component of $\partial \Delta_{\varepsilon}^{( \pm)}$that encircles a singular point $u_{*} \in \mathfrak{M}_{\text {sing }}^{( \pm)}$. In addition to $D=0$, there is a pole at $D=-i Q_{i} \varepsilon^{2}$ for each $Q_{i}$ satisfying (4.13) for $u=u_{*}$. In the limit $\varepsilon \rightarrow 0$, it collides with the pole at $D=0$. The other poles stay away from the real axis, even after the limit. Recall that the sign of such $Q_{i}$ is fixed: it is positive if $u_{*} \in \mathfrak{M}_{\text {sing }}^{(+)}$and negative if $u_{*} \in \mathfrak{M}_{\text {sing }}^{(-)}$. On the other hand, at $u \in \partial \mathfrak{M}$, there is one pole at $D=0$ and other poles are on the imaginary axis and are infinitely far away.

Let us first compute the contribution from the components of $\partial \Delta_{\varepsilon}^{(-)}$. (See figure 10 left.) No pole approaches $D=0$ from the lower half $D$ plane in the limit. Thus, the contour $\Gamma_{-}$can be deformed further away from $D=0$. In particular, we can take the $\varepsilon \rightarrow 0$ limit holding the contour $\Gamma_{-}$fixed. As a function of $u$, the integrand is continuous and its absolute value is bounded from above for any $\varepsilon$. Hence the integral vanishes in the $\varepsilon \rightarrow 0$ limit since the contour simply shrinks. Thus, we find

$$
\lim _{e \rightarrow 0} \cdot \lim _{\varepsilon \rightarrow 0} \int_{\Gamma_{-}} \mathrm{d} D \oint_{\partial \Delta_{\varepsilon}^{(-)}} \mathrm{d} u \frac{1}{D} g(u, D) \exp \left(-\frac{\pi}{e^{2}} D^{2}-2 \pi i \zeta D\right)=0 .
$$

Let us next consider the components of $\partial \Delta_{\varepsilon}^{(+)}$. (See figure 10 middle.) No pole approaches $D=0$ from the upper half $D$ plane, but one pole $D=-i Q_{i} \varepsilon^{2}$ for each $Q_{i}$ satisfying (4.13) approaches $D=0$ in the limit. And the contour $\Gamma_{\text {- goes between }} D=0$ and these poles. Therefore, we cannot take that limit holding the contour fixed. To avoid the complication, we decompose the contour as

$$
\Gamma_{-}=\Gamma_{+}+C_{0},
$$


where $C_{0}$ is a circle of radius smaller than $\varepsilon^{2}$ that goes around $D=0$ counter-clockwise. The integral along $\Gamma_{+}$vanishes in the limit for the same reason as in (4.36),

$$
\lim _{\substack{e \rightarrow 0 \\ \varepsilon \rightarrow 0}} \int_{\Gamma_{+}} \mathrm{d} D \oint_{\partial \Delta_{\varepsilon}^{(+)}} \mathrm{d} u \frac{1}{D} g(u, D) \exp \left(-\frac{\pi}{e^{2}} D^{2}-2 \pi i \zeta D\right)=0 .
$$

The integral along $C_{0}$, on the other hand, remains,

$$
\begin{aligned}
& \lim _{e \rightarrow 0} \cdot \lim _{\varepsilon \rightarrow 0} \int_{C_{0}} \mathrm{~d} D \oint_{\partial \Delta_{\varepsilon}^{(+)}} \mathrm{d} u \frac{1}{D} g(u, D) \exp \left(-\frac{\pi}{e^{2}} D^{2}-2 \pi i \zeta D\right) \\
& =\lim _{e \rightarrow 0} \cdot \lim _{\varepsilon \rightarrow 0} 2 \pi i \oint_{\partial \Delta_{\varepsilon}^{(+)}} \mathrm{d} u g(u, 0) \exp \left(-\frac{\pi}{e^{2}} 0^{2}-2 \pi i \zeta 0\right) \\
& =2 \pi i \oint_{\partial \Delta_{\varepsilon}^{(+)}} \mathrm{d} u g(u, 0) .
\end{aligned}
$$

Finally, let us study the contribution of $\partial \mathfrak{M}$. This is the main new point compared to the $2 \mathrm{~d}$ index computation [39] where $\mathfrak{M}$ was closed and had no infinity. In $1 \mathrm{~d}$, the index should undergo a transition as $\zeta$ crosses a wall, and it is the integral on $\partial \mathfrak{M}$ that is responsible for that transition. As we have discussed at the beginning of this section, the result should depend on the distance $|\Delta \zeta|$ to the wall in relation to $e$ and $\beta$. Since we are taking the limit $e \rightarrow 0$, we should also scale $\zeta$ accordingly. Here we present one particular scaling limit, which we shall call the Higgs scaling. (A more detailed discussion will be presented in the next subsection.) It is to scale up $|\zeta|$ as the limit $e \rightarrow 0$ is taken so that

$$
\zeta^{\prime}=e^{2} \zeta
$$

is held fixed. Then, the $D$ integral can be processed as follows:

$$
\begin{aligned}
& \int_{\Gamma_{-}} \frac{\mathrm{d} D}{D} g(u, D) \exp \left(-\frac{\pi}{e^{2}} D^{2}-2 \pi i \zeta D\right) \\
& =\int_{\Gamma_{-}} \frac{\mathrm{d} D^{\prime}}{D^{\prime}} g\left(u, e^{2} D^{\prime}\right) \exp \left(-\pi e^{2} D^{\prime 2}-2 \pi i \zeta^{\prime} D^{\prime}\right) \\
& \stackrel{e \rightarrow 0}{\longrightarrow} \int_{\Gamma_{-}} \frac{\mathrm{d} D^{\prime}}{D^{\prime}} g(u, 0) \exp \left(-2 \pi i \zeta^{\prime} D^{\prime}\right) \\
& =\left\{\begin{array}{ll}
0 & \zeta^{\prime}>0 \\
2 \pi i g(u, 0) & \zeta^{\prime}<0
\end{array}=2 \pi i \Theta\left(-\zeta^{\prime}\right) g(u, 0) .\right.
\end{aligned}
$$

Summing up the contributions, we find

$$
\begin{aligned}
I & =\frac{N_{1}}{2}\left[0-2 \pi i \oint_{\partial \Delta_{\varepsilon}^{(+)}} \mathrm{d} u g(u, 0)+2 \pi i \Theta\left(-\zeta^{\prime}\right) \oint_{\partial \mathfrak{M}} \mathrm{d} u g(u, 0)\right] \\
& =\mp N_{1} \pi i \oint_{\partial \Delta_{\varepsilon}^{( \pm)}} \mathrm{d} u g(u, 0) \quad \text { for } \pm \zeta^{\prime}>0 .
\end{aligned}
$$

If we choose $\Gamma=\Gamma_{+}$, then, we have non-zero contribution from $\partial \Delta_{\varepsilon}^{(-)}$, zero from $\partial \Delta_{\varepsilon}^{(+)}$, and similar (but opposite) contribution as (4.41) from $\partial \mathfrak{M}$. The sum is

$$
I=\frac{N_{1}}{2}\left[2 \pi i \oint_{\partial \Delta_{\varepsilon}^{(-)}} \mathrm{d} u g(u, 0)+0-2 \pi i \Theta\left(\zeta^{\prime}\right) \oint_{\partial \mathfrak{M}} \mathrm{d} u g(u, 0)\right]
$$




$$
=\mp N_{1} \pi i \oint_{\partial \Delta_{\varepsilon}^{( \pm)}} \mathrm{d} u g(u, 0) \quad \text { for } \pm \zeta^{\prime}>0,
$$

which is indeed the same as (4.42).

\subsection{Scaling limits}

In the evaluation of the integral along $\partial \mathfrak{M}$, we have taken the scaling limit where $e \rightarrow 0$, $|\zeta| \rightarrow \infty$ holding $e^{2} \zeta$ fixed at a non-zero value. To see the physical meaning of the limit, let us restore the circumference $\beta$ of the circle and also use the physical $D_{E}$ variable. The important factor of the integrand is

$$
\exp \left(-\frac{\beta}{2 e^{2}} D_{E}^{2}-i \beta \zeta D_{E}\right)
$$

Note that the exponent is indeed dimensionless, as the parameters and variables have the following dimensions (see the footnote in page 18):

$$
\left[D_{E}\right]=\text { energy }^{2}, \quad\left[e^{2}\right]=\text { energy }^{3}, \quad[\zeta]=\text { energy }^{-1}, \quad[\beta]=\text { energy }^{-1} .
$$

In any computation of path integral, it is natural to neutralize the dimension of integration variables. Since there are more than one dimensionful parameters, there are more than one ways to do so.

The scaling of the variable $D_{E}$ in the above computation is, when $\beta$ is restored,

$$
D_{E}=\beta e^{2} D_{E}^{\prime} .
$$

This $D_{E}^{\prime}$ is indeed dimensionless and the factor (4.44) is written as

$$
\exp \left(-\frac{\beta^{3} e^{2}}{2}\left(D_{E}^{\prime}\right)^{2}-i \beta^{2} e^{2} \zeta D_{E}^{\prime}\right)
$$

The limit we have taken is $e^{2} \beta^{3} \rightarrow 0$ holding $\zeta^{\prime}=\beta^{2} e^{2} \zeta$ fixed at a non-zero value. Even though $e$ vanishes, the D-term potential remains because $|\zeta|$ is sent to infinity at the same time. In particular, the Higgs mass $e \sqrt{|\zeta|}$ is kept finite and non-zero, in comparison to $1 / \beta$. Moreover, the potential barrier on the Coulomb branch is infinitely high, $e^{2} \zeta^{2} / 2=\left[\left(\zeta^{\prime}\right)^{2} /\left(2 e^{2} \beta^{3}\right)\right](1 / \beta)$. Therefore, this is a regular limit where the theory at the energies below $e^{2}|\zeta|=\zeta^{\prime} / \beta$ is well approximated by the theory on the Higgs branch. In particular, the obtained answer must agree with the index of the Higgs branch theory. That is why we called it the Higgs scaling.

Let us consider another scaling limit which attempts to probe the Coulomb branch dynamics, having characteristic energy scale $e^{2} \zeta^{2}$. That is to hold $\zeta^{\prime \prime}=\sqrt{\beta} e \zeta$ fixed when taking the limit $e^{2} \beta^{3} \rightarrow 0$. The natural rescaling of the variable $D_{E}$ is

$$
D_{E}=\frac{e}{\sqrt{\beta}} D_{E}^{\prime \prime}
$$

so that (4.44) is written as

$$
\exp \left(-\frac{1}{2}\left(D_{E}^{\prime \prime}\right)^{2}-i \sqrt{\beta} e \zeta D_{E}^{\prime \prime}\right)
$$


Then, the $D_{E}$ integration at infinity behaves as

$$
\begin{aligned}
& \int_{\Gamma} \frac{\mathrm{d} D_{E}}{D_{E}} g\left(u, \frac{\beta^{2}}{(2 \pi)^{2}} D_{E}\right) \exp \left(-\frac{\beta}{2 e^{2}} D_{E}^{2}-i \beta \zeta D_{E}\right) \\
& \quad=\int_{\Gamma} \frac{\mathrm{d} D_{E}^{\prime \prime}}{D_{E}^{\prime \prime}} g\left(u, \frac{\beta^{2} e}{(2 \pi)^{2} \sqrt{\beta}} D_{E}^{\prime \prime}\right) \exp \left(-\frac{1}{2}\left(D_{E}^{\prime \prime}\right)^{2}-i \zeta^{\prime \prime} D_{E}^{\prime \prime}\right) \\
& \stackrel{e \rightarrow 0}{\longrightarrow} \int_{\Gamma} \frac{\mathrm{d} D_{E}^{\prime \prime}}{D_{E}^{\prime \prime}} g(u, 0) \exp \left(-\frac{1}{2}\left(D_{E}^{\prime \prime}\right)^{2}-i \zeta^{\prime \prime} D_{E}^{\prime \prime}\right) .
\end{aligned}
$$

As long as $\Gamma$ avoids the pole at $D_{E}=0$ and asymptotes to the real line, this is absolutely convergent and depends continuously on $\zeta^{\prime \prime}$. If we choose $\Gamma=\Gamma_{-}$, it vanishes in the limit $\zeta^{\prime \prime} \rightarrow+\infty$ and approaches $2 \pi i g(0, u)$ in the opposite limit $\zeta^{\prime \prime} \rightarrow-\infty$. The difference from the limit value is exponentially small for large $\left|\zeta^{\prime \prime}\right|$ as $\pm \sqrt{2 \pi} i \mathrm{e}^{-\left|\zeta^{\prime \prime}\right|^{2} / 2} /\left|\zeta^{\prime \prime}\right|$ times $g(u, 0)$. Thus, in this scaling limit, the index is a continuous function of $\zeta^{\prime \prime}$ that interpolates the Higgs scaling result at $\zeta^{\prime}>0$ and the one at $\zeta^{\prime}<0$. According to a numerical evaluation of the integral, the transition occurs essentially within the range $\left|\zeta^{\prime \prime}\right|<5$.

This smooth transition appears to be different from the expectation (4.3) that we have a sharp transition within the range $\left|\zeta^{\prime \prime}\right| \ll 1$. This discrepancy can be understood by noting a subtlety in the definition of the index. ${ }^{6}$ Recall that, when there is a non-trivial wall crossing, the effective potential on the Coulomb branch approaches $E_{1}=e^{2}|\zeta|^{2} / 2=\left|\zeta^{\prime \prime}\right|^{2} / 2 \beta$ at infinity, which is finite in the scaling limit under consideration. In such a situation, the spectrum is continuous above $E_{1}=e^{2}|\zeta|^{2} / 2$. As long as the gap $E_{1}$ is positive, supersymmetric ground states must be normalizable, and the index can be defined unambiguously as

$$
\text { \#(bosonic zero energy states) - \#(fermionic zero energy states). }
$$

(Here we suppress the flavor twist for simplicity.) In particular, it is an integer and is deformation invariant. However, there is an ambiguity if we try to define the index as (4.1), that is,

$$
\operatorname{Tr}_{\mathcal{H}}(-1)^{F} \mathrm{e}^{-\beta H} \text {. }
$$

The expression (4.52) is not absolutely convergent due to the continuum above $E_{1}$, and hence depends on how it is defined. Our localization computation provides one definition to (4.52), but it may not agree with other possible definitions. In particular, it does not have to agree with (4.51). One may estimate the ambiguity to be of the order of $\mathrm{e}^{-\beta E_{1}}=\mathrm{e}^{-\left|\zeta^{\prime \prime}\right|^{2} / 2}$. That is in fact the order of the difference of (4.50) from the Higgs scaling result, as estimated above at large $\left|\zeta^{\prime \prime}\right|$.

In the Higgs scaling, on the other hand, the asymptotic gap $E_{1}=\left|\zeta^{\prime}\right|^{2} /\left(2 e^{2} \beta^{4}\right)$ blows up to infinity as remarked earlier. Hence, (4.52) is absolutely convergent and must agree with (4.51).

It is the index as defined by (4.51) that is expected to undergo a sharp transition by the runaway of Coulomb branch vacua. Since it is deformation invariant as long as the gap $E_{1}$ is bounded from below by a positive energy, the total jump of our localization result must agree with the one from the picture of runaway vacua. In fact, we shall explicitly

\footnotetext{
${ }^{6}$ We thank Edward Witten for a guide on this point.
} 
show the agreement in section 5 whenever the wall crossing on the Coulomb branch can be evaluated.

We now move on to the computation of the index of the theory with a general gauge group. We shall take the "Higgs scaling": $e^{2} \beta^{3} \rightarrow 0$ holding the distance of $\zeta^{\prime}=\beta^{2} e^{2} \zeta$ to the walls fixed.

\subsection{Higher rank theories}

We set $\beta=2 \pi$. We define $G(u, D):=N_{\ell}\left(-\frac{1}{2}\right)^{\ell} g(u, D) \mathrm{e}^{-\frac{\pi}{e^{2}} D^{2}-2 \pi i \zeta(D)}$ and consider it as a function of $(u, D) \in \tilde{\mathfrak{M}} \times \mathfrak{t}_{\mathbf{C}}$. Let us introduce a $\mathfrak{t}_{\mathbf{C}}^{*}$-valued one form $\nu$ on $\tilde{\mathfrak{M}} \times \mathfrak{t}_{\mathbf{C}}$ by

$$
\nu(\xi):=-i \mathrm{~d} \bar{u}_{a} h^{a b} \xi_{b}, \quad \xi \in \mathfrak{t}_{\mathbf{C}} .
$$

Both $G$ and $\nu$ are meromorphic in $D$. In terms of these, the index formula (4.29) reads

$$
I=\frac{1}{|W|} \lim \int_{\left(\tilde{\mathfrak{M} \backslash} \backslash \Delta_{\varepsilon}\right) \times \Gamma} \mu, \quad \mu:=G \mathrm{~d}^{\ell} u \wedge(\nu(\mathrm{d} D))^{\wedge \ell} .
$$

$\Gamma \subset \mathfrak{t}_{\mathbf{C}}$ is a contour of $D$-integration. The real locus $\Gamma=i \mathfrak{t}$ was chosen in (4.29), but we shall deform it away so as not to hit the poles of the integrand, as in the U(1) case. The identity (4.31) reads

$$
\bar{\partial} G=\nu(D) G .
$$

One can easily show that $\partial_{\bar{u}_{c}} h^{a b}$ is symmetric in $a, b, c$, which means

$$
\bar{\partial} \nu=0
$$

For a set $\left\{Q_{1}, \ldots, Q_{s}\right\} \subset \mathfrak{t}^{*}$, put

$$
\mu_{Q_{1}, \ldots, Q_{s}}:=c_{s} \cdot G \mathrm{~d}^{\ell} u \wedge(\nu(\mathrm{d} D))^{\wedge(\ell-s)} \wedge \frac{\mathrm{d} Q_{1}(D)}{Q_{1}(D)} \wedge \cdots \wedge \frac{\mathrm{d} Q_{s}(D)}{Q_{s}(D)},
$$

with $c_{s}=(-1)^{s(\ell-1)+\frac{s(s+1)}{2}}$. Here $Q_{\alpha}$ 's may or may not be taken from the charges of the fields. Note that it vanishes if $Q_{1}, \ldots, Q_{s}$ are linearly dependent. For the empty set, we have $\mu_{\emptyset}=\mu$. It follows from (4.55) and (4.56) that

$$
\mathrm{d} \mu_{Q_{1}, \ldots, Q_{s}}=\sum_{\alpha=1}^{s}(-1)^{s-\alpha} \mu_{Q_{1}, \ldots \widehat{Q_{\alpha}} \ldots, Q_{s}},
$$

where "hat" means omission.

We shall use this identity and Stokes theorem successively to reduce the dimension of the $u$ integration. To show the idea, let us pretend that $G$ and $\nu$ had no-singularity and consider integration on $\tilde{\mathfrak{M}}$ rather than $\tilde{\mathfrak{M}} \backslash \Delta_{\varepsilon}$. (We ignore the $D$ integration for now.) We also pretend that $\tilde{\mathfrak{M}}$ is compact without boundary for now. We choose a good cell decomposition of $\tilde{\mathfrak{M}}$. By "good" we mean that a codimension $k$ cell is at the intersection of $(k+1)$ codimension $(k-1)$ cells (e.g. codimension 1 cell is at the intersection of 2 maximal dimensional cells). Write $\left\{C_{\alpha}^{(n)}\right\}_{\alpha}$ for the set of all $n$-dimensional cells. We 
choose, randomly, an element $Q_{\alpha} \in i \mathfrak{t}^{*}$ for each maximal dimensional cell $C_{\alpha}^{(2 \ell)}$ and use $\mu=\mathrm{d} \mu_{Q_{\alpha}}$ there. Then,

$$
\begin{aligned}
\int_{\tilde{\mathfrak{M}}} \mu & =\sum_{\alpha} \int_{C_{\alpha}^{(2 \ell)}} \mathrm{d} \mu_{Q_{\alpha}}=\sum_{\alpha} \int_{\partial C_{\alpha}^{(2 \ell)}} \mu_{Q_{\alpha}} \\
& =\sum_{\beta} \int_{C_{\beta}^{(2 \ell-1)}}\left(\mu_{Q_{\beta^{+}}}-\mu_{Q_{\beta^{-}}}\right)=\sum_{\beta} \int_{C_{\beta}^{(2 \ell-1)}} \mathrm{d} \mu_{Q_{\beta^{+}}, Q_{\beta^{-}}}=\sum_{\beta} \int_{\partial C_{\beta}^{(2 \ell-1)}} \mu_{Q_{\beta^{+}}, Q_{\beta^{-}}} \\
& =\sum_{\gamma} \int_{C_{\gamma}^{(2 \ell-2)}}\left(\mu_{Q_{\gamma^{1}}, Q_{\gamma^{2}}}-\mu_{Q_{\gamma^{1}}, Q_{\gamma^{3}}}+\mu_{Q_{\gamma^{2}}, Q_{\gamma^{3}}}\right)=\sum_{\gamma} \int_{\partial C_{\gamma}^{(2 \ell-2)}} \mu_{Q_{\gamma^{1}}, Q_{\gamma^{2}}, Q_{\gamma^{3}}} \\
& =\cdots
\end{aligned}
$$

In this way, we can lower the dimension of $u$-integration, and eventually we hit the middle dimension, i.e., $\ell$. There we have complete cancellation because of the identity (4.58) and $\mu_{Q_{\gamma_{1}}, \ldots, Q_{\gamma_{\ell+1}}}=0$. Thus, we get zero.

Now let us consider the real situation where $\tilde{\mathfrak{M}}$ is non-compact and we delete $\Delta_{\varepsilon}$ from $\tilde{\mathfrak{M}}$. To avoid possible misunderstanding, we denote by $\mathscr{I}_{S}$ the set labelling the singular hyperplanes. $\mathscr{I}_{S}$ is different from the set I labelling the weight decomposition (4.12) of $V_{\text {chiral }}$ since different components may define the same singular hyperplane. Thus, (4.14) could also be written as

$$
\Delta_{\varepsilon}:=\bigcup_{i \in \mathscr{I}_{S}} \Delta_{\varepsilon}\left(H_{i}\right)
$$

We consider the non-degenerate case: all the intersections of hyperplanes are transversal. In particular, there is no point where distinct $(\ell+1)$ hyperplanes meet. The boundary $\partial \Delta_{\varepsilon}$ is separated into tubes with holes,

$$
S_{i}:=\partial \Delta_{\varepsilon} \cap \partial \Delta_{\varepsilon}\left(H_{i}\right) .
$$

We give it the natural orientation. We also introduce a cut-off at infinity, $\tilde{\mathfrak{M}}_{R}:=i \mathfrak{t} / \mathrm{Q}^{\vee} \times \mathfrak{t}_{R}$, where $\mathfrak{t}_{R}$ consists of vectors of lengths $\leq R$. In (4.54), we replace $\tilde{\mathfrak{M}}$ by $\tilde{\mathfrak{M}}_{R}$ and send $R$ to infinity at the end of the computation. We put

$$
S_{\infty}:=-\overline{\left(\partial \tilde{\mathfrak{M}}_{R}\right) \backslash \Delta_{\varepsilon}}
$$

Note that

$$
\partial\left(\tilde{\mathfrak{M}}_{R} \backslash \Delta_{\varepsilon}\right)=-\sum_{i \in \mathscr{I}} S_{i}
$$

Here and in what follows, unless otherwise stated, the index $i$ runs over $\mathscr{I}=\mathscr{I}_{S} \cup\{\infty\}$. I.e., $i$ may be the label of a singular hyperplane or $i=\infty$. We write $S_{i_{1} \ldots i_{s}}=S_{i_{1}} \cap \cdots \cap S_{i_{s}}$ and we also give it the natural orientation. It is totally antisymmetric in $i_{1}, \ldots, i_{s}$ and

$$
\partial S_{i_{1} \ldots i_{s}}=-\sum_{j \in \mathscr{I}} S_{i_{1} \ldots i_{s} j}
$$

We would like to choose a cell decomposition of $\tilde{\mathfrak{M}}_{R} \backslash \Delta_{\varepsilon}$. Because it is a manifold with boundary and corners, we cannot take a good one, but we try to take it to be as 
good as possible. We require the following conditions (i)-(iii). (i) Each open cell is either in the interior or contained in the interior of exactly one $S_{i_{1} \ldots i_{s}}$. (ii) It is good in the interior of $\tilde{\mathfrak{M}}_{R} \backslash \Delta_{\varepsilon}$. That is, the valence condition is satisfied at the (closed) cell in the interior. To describe the final condition, we note that a neighborhood of $\tilde{\mathfrak{M}}_{R} \backslash \Delta_{\varepsilon}$ of an interior point of a corner $S_{i_{1} \ldots i_{s}}$ is of the form $\left(\mathbf{R}_{+}\right)^{s} \times \mathbf{R}^{2 \ell-s}$, which is the domain in $\mathbf{R}^{2 \ell}=$ $\left\{\left(x_{i_{1}}, \ldots, x_{i_{s}}, y_{s+1}, \ldots, y_{2 \ell}\right)\right\}$ defined by $x_{i_{1}} \geq 0, \ldots, x_{i_{s}} \geq 0$. For $\left\{j_{1}, \ldots, j_{p}\right\} \subset\left\{i_{1}, \ldots, i_{s}\right\}$, the corner $S_{j_{1} \ldots j_{p}}$ includes $S_{i_{1} \ldots i_{s}}$ and is identified as the region $x_{j_{1}}=\cdots=x_{j_{p}}=0$. We introduce a cell decomposition of $\left(\mathbf{R}_{+}\right)^{s} \times \mathbf{R}^{2 \ell-s}$ as follows. For $\left\{j_{1}, \ldots, j_{p}\right\} \subset\{1, \ldots, s\}$, we define the cell $C_{j_{1} \ldots j_{p}}$ by the condition.

$$
0 \leq x_{j_{1}}=\cdots=x_{j_{p}} \leq \text { all other } x_{i}{ }^{\prime} \text { s. }
$$

Note that the boundary of $C_{j_{1} \ldots j_{p}}$ consists of $S_{j_{1} \ldots j_{p}}$ and $C_{j_{1} \ldots j_{p} i}$. The orientation of $C_{j}$ is the one induced from $\tilde{\mathfrak{M}}$ and we can assign an orientation of $C_{j_{1} \ldots j_{p}}$ so that

$$
\partial C_{j_{1} \ldots j_{p}}=-S_{j_{1} \ldots j_{p}}+\sum_{i} C_{j_{1} \ldots j_{p} i}
$$

We can now describe the condition (iii): a cell touching the interior of $S_{i_{1} \ldots i_{s}}$ coincides with one of $C_{j_{1} \ldots j_{p}}$, or its subdivision in the $\mathbf{R}^{2 \ell-s}$ direction, in a neighborhood of $S_{i_{1} \ldots i_{s}}$.

Under such a cell decomposition, we compute the integral $\int_{\tilde{\mathfrak{M}}_{R} \backslash \Delta_{\varepsilon}} \mu$ as follows. To each maximal dimensional cell, we assign an element of $i t^{*}$. For a cell away from the boundary, the assignment is random. If it touches the interior of $S_{i}$, with $i \in \mathscr{I}_{S}$, we assign the charge $Q_{i}$ of a field defining $H_{i}$. If it touches the interior of $S_{\infty}$, we assign an element $Q_{\infty} \in i t^{*}$ which may or may not be one of the charges. More generally, if it touches the interior of $S_{i_{1} \ldots i_{s}}$, it must be contained in one of $C_{j}$ for $j \in\left\{i_{1}, \ldots, i_{s}\right\}$, then we assign $Q_{j}$. The integral in the interior region can be processed as in (4.59), and we obtain zero. From the cells touching the boundary, we have other contributions. From the cells touching the interior $S_{i}^{o}$ of $S_{i}$, we have

$$
\sum_{C_{\beta}^{(2 \ell-1)} \subset S_{i}^{o}} \int_{C_{\beta}^{(2 \ell-1)}} \mu_{Q_{i}} .
$$

To see what you obtain from the cells touching the interior of higher codimension corners, let us look at the region $\left(\mathbf{R}_{+}\right)^{s} \times \mathbf{R}^{2 \ell-s}$ around $S_{i_{1} \ldots i_{s}}$.

$$
\begin{aligned}
\int_{\left(\mathbf{R}_{+}\right)^{s} \times \mathbf{R}^{2 \ell-s}} \mu & =\sum_{i} \int_{C_{i}} \mathrm{~d} \mu_{Q_{i}}=\sum_{i} \int_{\partial C_{i}} \mu_{Q_{i}} \\
= & \sum_{i} \int_{-S_{i}} \mu_{Q_{i}}+\sum_{i} \sum_{j \neq i} \int_{C_{i j}} \mu_{Q_{i}}+[\cdots] \\
& \underline{\downarrow} \sum_{i} \int_{-S_{i}} \mu_{Q_{i}}+\sum_{i<j} \int_{\partial C_{i j}} \mu_{Q_{i}, Q_{j}}+[\cdots] \\
= & \sum_{i} \int_{-S_{i}} \mu_{Q_{i}}+\sum_{i<j} \int_{-S_{i j}} \mu_{Q_{i}, Q_{j}}+\sum_{i<j} \sum_{k \neq i, j} \int_{C_{i j k}} \mu_{Q_{i}, Q_{j}}+[\cdots] \\
& =\cdots
\end{aligned}
$$




$$
=-\sum_{i} \int_{S_{i}} \mu_{Q_{i}}-\sum_{i<j} \int_{S_{i j}} \mu_{Q_{i}, Q_{j}}-\cdots-\int_{S_{i_{1} \ldots i_{s}}} \mu_{Q_{i_{1}}, \ldots, Q_{i_{s}}}+[\cdots],
$$

where we used (4.58) as well as (4.66) in the intermediate steps. For example, we used the following in $\stackrel{\downarrow}{=}$

$$
\sum_{i} \sum_{j \neq i} \int_{C_{i j}} \mu_{Q_{i}}=\sum_{i<j} \int_{C_{i j}}\left(\mu_{Q_{i}}-\mu_{Q_{j}}\right)=\sum_{i<j} \int_{C_{i j}} \mathrm{~d} \mu_{Q_{i}, Q_{j}}=\sum_{i<j} \int_{\partial C_{i j}} \mu_{Q_{i}, Q_{j}} .
$$

The terms $+[\cdots]$ that appear after partial integration consists of the boundary terms in the interior, which will contribute to the complete cancellation as in (4.59). Collecting all these, we have

$$
\int_{\left(\tilde{\left.\mathfrak{M} \backslash \Delta_{\varepsilon}\right) \times \Gamma}\right.} \mu=-\sum_{i} I_{i}-\sum_{i<j} I_{i j}-\cdots-\sum_{i_{1}<\cdots<i_{\ell}} I_{i_{1} \ldots i_{\ell}}=-\sum_{p=1}^{\ell} \sum_{i_{1}<\cdots<i_{p}} I_{i_{1} \ldots i_{p}},
$$

where

$$
I_{i_{1} \ldots i_{p}}=\int_{S_{i_{1} \ldots i_{p} \times \Gamma}} \mu_{Q_{i_{1}} \ldots Q_{i_{p}}} .
$$

We emphasize again that the index $i_{a}$ runs over $\mathscr{I}=\mathscr{I}_{S} \cup\{\infty\}$.

\subsection{The $D$-contour}

So far, we have focused our attention to the integration over $\tilde{\mathfrak{M}}_{R} \backslash \Delta_{\varepsilon}$. Let us now bring the $D$-integration back into our consideration. An appropriate choice of the contour $\Gamma$ will allow us to process the integral further. Our original choice is $\Gamma=i t^{*}$ with a fixed orientation. We may shift it to

$$
\Gamma=i \delta+i \mathfrak{t}
$$

for some $\delta \in i$ t which is small enough so that the integrand $\mu$ remains non-singular over $\tilde{\mathfrak{M}} \backslash \Delta_{\varepsilon}$ as the contour is shifted from $i$ to $i \delta+i$ t. Let us fix such a $\delta$ which is generic enough so that $Q_{i}(\delta)$ is non-zero for any $i \in \mathscr{I}$. Of course, the final result does not depend on the choice of $\delta$, but each term $I_{i_{1} \ldots i_{p}}$ in the expansion (4.70) may depend on it. So let us denote it by $I_{i_{1} \ldots i_{p}}(\delta)$.

Let us look at the term $I_{i_{1} \ldots i_{p}}(\delta)$ where all the indices are from $\mathscr{I}_{S}$. If $Q_{i_{a}}(\delta)>0$ for some $i_{a}$, the integrand is regular even if the neighborhood $\Delta_{\varepsilon}\left(H_{i_{a}}\right)$ is shrunk to the zero size. Therefore, $I_{i_{1} \ldots i_{p}}(\delta)$ vanishes in the $\varepsilon \rightarrow 0$ limit. If $Q_{i_{a}}(\delta)<0$ for all of $i_{1}, \ldots, i_{p}$, we deform $\Gamma$ upward in each $Q_{i_{a}}(D)$-plane so that it decomposes into a union of the infinite line in the upper-half plane and the component that encircles the origin. The former vanishes in the $\varepsilon \rightarrow 0$ limit, while the latter picks up the simple pole at $Q_{i_{a}}(D)=0$. We are therefore left with the $D$-integration along the common kernel $\operatorname{Ker} Q_{i_{1} \ldots i_{p}}$ of all $Q_{i_{a}}$ 's. Here, just as in (4.72), there is a freedom to set the new contour at

$$
i \delta_{i_{1} \ldots i_{p}}+\operatorname{Ker} Q_{i_{1} \ldots i_{p}}
$$


where $\delta_{i_{1} \ldots i_{p}} \in \operatorname{Ker} Q_{i_{1} \ldots i_{p}}$ is small enough. The result does not depend on the choice of $\delta_{i_{1} \ldots i_{p}}$. Taking one step backward, we may write the result as

$$
I_{i_{1} \ldots i_{p}}(\delta)=\prod_{a=1}^{p} \theta\left(-Q_{i_{a}}(\delta)\right) \int_{S_{i_{1} \ldots i_{p}} \times \Gamma_{i_{1} \ldots i_{p}}} \mu_{Q_{i_{1}} \ldots Q_{i_{p}}}
$$

where $\theta(x)$ is the step function ( 1 on $x>0$ and 0 on $x<0)$ and

$$
\Gamma_{i_{1} \ldots i_{p}}:=C_{i_{1} \ldots i_{p}} \times\left(i \delta_{i_{1} \ldots i_{p}}+\operatorname{Ker} Q_{i_{1} \ldots i_{p}}\right) .
$$

Here $C_{i_{1} \ldots i_{p}} \subset \mathfrak{t}_{\mathbf{C}}$ is any $p$-dimensional contour whose $Q_{i_{a}}$-image is a circle around the origin. We provide $\Gamma_{i_{1} \ldots i_{p}}$ the orientation that is induced from $\Gamma$ in (4.72) by the above deformation.

The integrals in (4.74) can be processed further. Let us take $\left\{Q_{j_{1}}, \ldots, Q_{j_{s}}\right\} \subset i \mathrm{t}^{*}$ so that the $p+s$ elements $Q_{i_{1}}, \ldots, Q_{i_{p}}, Q_{j_{1}}, \ldots, Q_{j_{s}}$ are linearly independent. We have from (4.58),

$$
\mathrm{d} \mu_{Q_{i_{1}} \ldots Q_{i_{p}} Q_{j_{1}} \ldots Q_{j_{s}}}=\sum_{a=1}^{p}(-1)^{p+s-a} \mu_{Q_{i_{1}} \ldots \widehat{Q_{i_{a}} \ldots Q_{j_{s}}}}+\sum_{b=1}^{s}(-1)^{s-b} \mu_{Q_{i_{1}} \ldots \widehat{Q_{j_{b}}} \ldots Q_{j_{s}}} .
$$

When integrated over $\Gamma_{i_{1} \ldots i_{p}}$, the first $p$ terms of (4.76) vanish since the $a$-th term does not have a pole at $Q_{i_{a}}(D)=0$ : for any chain $C$ of $\tilde{\mathfrak{M}}_{R} \backslash \Delta_{\varepsilon}$,

$$
\int_{C \times \Gamma_{i_{1} \ldots i_{p}}} \mathrm{~d} \mu_{Q_{i_{1}} \ldots Q_{i_{p}} Q_{j_{1}} \ldots Q_{j_{s}}}=\sum_{b=1}^{s}(-1)^{s-b} \int_{C \times \Gamma_{i_{1} \ldots i_{p}}} \mu_{Q_{i_{1}} \ldots Q_{i_{p}} Q_{j_{1}} \ldots \widehat{Q_{j_{b}} \ldots Q_{j_{s}}}} .
$$

In the same way as we obtained (4.70) using a cell decomposition of $\tilde{\mathfrak{M}}_{R} \backslash \Delta_{\varepsilon}$ and the identity (4.58), we find, via a cell decomposition of $S_{i_{1} \ldots i_{p}}$ and the identity (4.77),

$$
\int_{S_{i_{1} \ldots i_{p}} \times \Gamma_{i_{1} \ldots i_{p}}} \mu_{Q_{i_{1}} \ldots Q_{i_{p}}}=-\sum_{q=p+1}^{\ell} \sum_{i_{p+1}<\cdots<i_{q}} I_{i_{1} \ldots i_{p} ; i_{p+1} \ldots i_{q}}\left(\delta_{i_{1} \ldots i_{p}}\right),
$$

where

$$
I_{i_{1} \ldots i_{p} ; i_{p+1} \ldots i_{q}}\left(\delta_{i_{1} \ldots i_{p}}\right)=\int_{S_{i_{1} \ldots i_{q}} \times \Gamma_{i_{1} \ldots i_{p}}} \mu_{Q_{i_{1} \ldots Q_{i_{q}}}} .
$$

The total sum (4.78) is independent of the choice of $\delta_{i_{1} \ldots i_{p}}$ but the individual term (4.79) may depend on the choice. Let us assume that $Q_{j}\left(\delta_{i_{1} \ldots i_{p}}\right) \neq 0$ for all charges $Q_{j}$ which are linearly independent of $Q_{i_{1}}, \ldots, Q_{i_{p}}$. Then, the term where all of $i_{p+1}, \ldots i_{q}$ are from $\mathscr{I}_{S}$ can be processed in the same way.

Repeating this procedure enough number of times, the index can be written as the sum of integrations of the form

$$
\int_{S_{i_{1} \ldots i_{\ell}} \times \Gamma_{i_{1} \ldots i_{\ell}}} \mu_{Q_{i_{1}} \ldots Q_{i_{\ell}}}, \quad \text { for }\left\{i_{1}, \ldots, i_{\ell}\right\} \subset \mathscr{I}_{S},
$$

plus the sum of integrations at infinity of the form

$$
\int_{S_{i_{1} \ldots i_{q} \infty} \times \Gamma_{i_{1} \ldots i_{p}}} \mu_{Q_{i_{1}} \ldots Q_{i_{q}} Q_{\infty}, \quad 0 \leq p \leq q<\ell .}
$$


The integration at infinity (4.81) depends on the value of $\zeta$ as well as the $D$-contour. It is not always possible to simplify the expression. In each example which we studied, however, as long as we take the scaling limit $e \rightarrow 0, \zeta \rightarrow \infty$, keeping $\zeta^{\prime}=e^{2} \zeta$ fixed at a generic value, those unprocessible expressions either vanish or cancel among each other, and we find that the final result for the index is a sum of the terms of the form (4.80).

\subsection{A systematic procedure}

In fact, there is a systematic procedure to find a concise answer. It is to apply the procedure employed in [40] involving a choice of $\eta \in i \mathrm{t}^{*}$ which is $\mathscr{I}_{S}$-generic, that is, $\eta$ cannot be

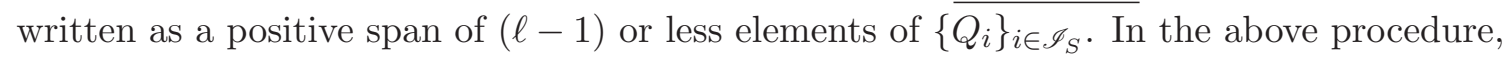
we have successively chosen the shift parameter $\delta$...

$$
\delta \rightarrow \delta_{i_{1} \ldots i_{p}} \rightarrow \delta_{i_{1} \ldots i_{p} ; i_{p+1} \ldots i_{q}} \rightarrow \delta_{i_{1} \ldots i_{p} ; i_{p+1} \ldots i_{q} ; i_{q+1} \ldots i_{r}} \rightarrow \cdots
$$

The choice was random, and in general route dependent: $\delta_{i_{1} \ldots i_{p} ; i_{p+1} \ldots i_{q}}$ does not have to be the same as $\delta_{j_{1} \ldots j_{s} ; j_{s+1} \ldots j_{q}}$ even if $\left\{i_{1}, \ldots, i_{q}\right\}=\left\{j_{1}, \ldots, j_{q}\right\}$. One feature of the systematic procedure is to remove the route dependence:

$$
\delta_{i_{1} \ldots i_{p_{1}} ; i_{p_{1}+1 \ldots i} \ldots i_{p_{2}} ; \cdots ; i_{p_{l}+1 \ldots i_{q}}}=\delta_{i_{1} \ldots i_{q}} .
$$

We recall that $\delta_{i_{1} \ldots i_{p}}$ is chosen for each subset $\left\{i_{1}, \ldots, i_{p}\right\}$ of $\mathscr{I}_{S}$ such that $Q_{i_{1}}, \ldots, Q_{i_{p}}$ are linearly independent: it vanishes on $Q_{i_{1}}, \ldots, Q_{i_{p}}$, and takes a non-zero value on each charge $Q_{j}$ which is linearly independent of $Q_{i_{1}}, \ldots, Q_{i_{p}}$. (The subset may be empty, for which $\delta_{\emptyset}=\delta$.) We also introduce a new notation:

$$
I_{i_{1} \ldots i_{p}}\left[\delta_{i_{1} \ldots i_{p}}\right]:=\int_{S_{i_{1} \ldots i_{p}} \times \Gamma_{i_{1} \ldots i_{p}}} \mu_{Q_{i_{1}} \ldots Q_{i_{p}}} .
$$

Here $\Gamma_{i_{1} \ldots i_{p}}$ is defined as a set by (4.75), and is provided the orientation which is induced from the orientation of $\Gamma=i \delta^{\prime}+i \mathrm{t}^{*}$ by deformation, where $\delta^{\prime} \in i \mathrm{t}^{*}$ has negative values on $Q_{i_{1}}, \ldots, Q_{i_{p}}$. Note that the orientation does not depend on the order of $i_{1}, \ldots, i_{p}$. The notation is redundant - the integral does not really depend on the choice of $\delta_{i_{1} \ldots i_{p}}$. For example, in the new notation, the index $I$ itself should be written as $I[\delta]-\delta$ enters into the integration contour $\Gamma$ via (4.72) but the integral does not depend on it. However, this notation is useful to keep track of what happens next. As in (4.70) and (4.78), we have

$$
\begin{aligned}
I_{i_{1} \ldots i_{p}}\left[\delta_{i_{1} \ldots i_{p}}\right]=-\sum_{q=p+1}^{\ell} & \left(\sum_{\substack{i_{p+1}<\cdots<i_{q} \\
\text { in } \mathscr{I}_{S}}} \prod_{a=p+1}^{q} \theta\left(-Q_{i_{a}}\left(\delta_{i_{1} \ldots i_{p}}\right)\right) \cdot I_{i_{1} \ldots i_{q}}\left[\delta_{i_{1} \ldots i_{q}}\right]\right. \\
& \left.+\sum_{\substack{i_{p+1}<\cdots<i_{q-1} \\
\text { in } \mathscr{I}_{S}}} \prod_{a=p+1}^{q-1} \theta\left(-Q_{i_{a}}\left(\delta_{i_{1} \ldots i_{p}}\right)\right) \cdot I_{i_{1} \ldots i_{q-1} \infty}\left[\delta_{i_{1} \ldots i_{q-1}}\right]\right),
\end{aligned}
$$

where

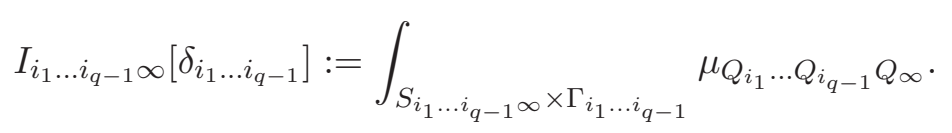


The main ingredient of the systematic procedure of [40] is the choice of $\eta \in i t^{*}$. We require all $\delta_{i_{1} \ldots i_{p}}$ to have negative values on $\eta$ :

$$
\eta\left(\delta_{i_{1} \ldots i_{p}}\right)<0, \quad \forall\left\{i_{1}, \ldots, i_{p}\right\} .
$$

In the present case of $1 \mathrm{~d}$ index, it is particularly useful to set

$$
\eta=\zeta \text { and } Q_{\infty}=\zeta .
$$

This is a valid choice if and only if $\zeta$ is $\mathscr{I}_{S^{-}}$generic. For this choice, the integration at infinity (4.86) involves the integration over $Q_{\infty}(D)=\zeta(D)$ of the following type:

$$
\int_{\zeta\left(\Gamma_{i_{1} \ldots i_{q-1}}\right)} \frac{\mathrm{d} \zeta(D)}{\zeta(D)} f(\ldots, \zeta(D)) \exp \left(-\frac{\pi}{e^{2}} D^{2}-2 \pi i \zeta(D)\right) .
$$

Note that (4.87) and $\eta=\zeta$ means $\zeta\left(\delta_{i_{1} \ldots i_{q-1}}\right)<0$, which means that the contour of integration (4.89) goes below the pole $\zeta(D)=0$. Hence, just as in the $\mathrm{U}(1)$ case (4.41), the integral vanishes in the Higgs scaling: $e \rightarrow 0, \zeta \rightarrow \infty$, keeping $\zeta^{\prime}=e^{2} \zeta$ finite. Therefore, the integration at infinity (4.86) all vanish!

Thus, in the recursion relation (4.85) we are left with the bulk terms only. As in [40], this recursion relation leads to the following result:

$$
I=\frac{1}{|W|} \sum_{\substack{i_{1}<\ldots<i_{\ell} \\ \text { in } \mathscr{I}_{S}}}(-1)^{\ell} \prod_{j \in\left\{i_{1}, \ldots, i_{\ell}\right\}} \theta\left(-Q_{j}\left(\delta_{i_{1} \ldots \hat{j} \ldots i_{\ell}}\right)\right) \int_{S_{i_{1} \ldots i_{\ell} \times \Gamma_{i_{1} \ldots i_{\ell}}}} \mu_{Q_{i_{1}} \ldots Q_{i_{\ell}}} .
$$

The step function factor is non-zero if and only if $\zeta=\eta$ belongs to the cone $\mathcal{C}_{Q_{1} \ldots Q_{i_{\ell}}}$ spanned by $Q_{i_{1}}, \ldots, Q_{i_{\ell}}$. Therefore, the index can also be written as

$$
\begin{aligned}
I & =\frac{1}{|W|} \sum_{\zeta \in \mathcal{C}_{Q_{1} \ldots Q_{i_{\ell}}}} I_{i_{1} \ldots i_{\ell}}, \\
I_{i_{1} \ldots i_{\ell}} & :=(-1)^{\ell} \int_{S_{i_{1} \ldots i_{\ell} \times \Gamma_{i_{1} \ldots i_{\ell}}}} \mu_{Q_{i_{1} \ldots Q_{i_{\ell}}}}=\epsilon_{i_{1} \ldots i_{\ell}} \int_{S_{i_{1} \ldots i_{\ell}}} C_{\ell} g(u, 0) \mathrm{d}^{\ell} u,
\end{aligned}
$$

where $C_{\ell}:=N_{\ell}(-1)^{\frac{\ell(\ell+1)}{2}}(\pi i)^{\ell}$ and

$$
\int_{\Gamma_{i_{1} \ldots i_{\ell}}} \frac{\mathrm{d} Q_{i_{1}}(D)}{Q_{i_{1}}(D)} \wedge \cdots \wedge \frac{\mathrm{d} Q_{i_{\ell}}(D)}{Q_{i_{\ell}}(D)}=(2 \pi i)^{\ell} \epsilon_{i_{1} \ldots i_{\ell}} .
$$

In view of the orientation of $\Gamma_{i_{1} \ldots i_{\ell}}$ as described below Eqn (4.84), $\epsilon_{i_{1} \ldots i_{\ell}}$ is a sign that is antisymmetric in $i_{1}, \ldots, i_{\ell}$ and odd under $Q_{i_{1}} \rightarrow-Q_{i_{1}}$. Recall also that $S_{i_{1} \ldots i_{\ell}}$ is antisymmetric in $i_{1}, \ldots, i_{\ell}$ and even under $Q_{i_{1}} \rightarrow-Q_{i_{1}}$. The product $\epsilon_{i_{1} \ldots i_{\ell}} S_{i_{1} \ldots i_{\ell}}$ is hence symmetric in $i_{1}, \ldots, i_{\ell}$ and odd under $Q_{i_{1}} \rightarrow-Q_{i_{1}}$. This together with the constraint that $\zeta$ must belong to the cone $\mathcal{C}_{Q_{i_{1}} \ldots Q_{i_{\ell}}}$ is the defining property of the JK residue [45] (See [40] for a review). Therefore, the index is the sum of JK residue of $C_{\ell} g(u, 0) \mathrm{d}^{\ell} u$ with respect to $\zeta$ at the isolated intersections of the singular hyperplanes. In the notation of [40], it reads

$$
I=\frac{1}{|W|} \sum_{\substack{i_{1}<\cdots<i_{\ell} \\ \text { in } \mathscr{I}_{S}}} \sum_{p \in H_{i_{1}} \cap \cdots \cap H_{i_{\ell}}} \operatorname{JK}-\operatorname{Res}\left(\left\{Q_{i_{1}}, \ldots, Q_{i_{\ell}}\right\}, \zeta\right)\left[C_{\ell} g(u, 0) \mathrm{d}^{\ell} u\right] .
$$




\subsection{The result}

We now fix the normalization constant $N_{\ell}$. The right choice turns out to be such that $C_{\ell}= \pm 1$, where the choice of sign depends on the definition of $(-1)^{F}$. As we will see in the next section, this choice yields the wall crossing formula that matches with the Coulomb branch result. To simplify the notation, we shall write $C_{\ell} g(u, 0)$ as $g$, or $g(u, z)$ if we want to make the parameter dependence explicit. Also, we may write the result (4.94) simply as

$$
I=\frac{1}{|W|} \operatorname{JK}-\operatorname{Res}_{\zeta} g \mathrm{~d}^{\ell} u .
$$

For $C_{\ell}=1$, we have

$$
\begin{aligned}
g & =\prod_{\alpha} 2 i \sin (-\pi \alpha(u)) \frac{\prod_{j \in \mathrm{J}} 2 i \sin \left(-\pi\left(q_{i}(u)+q_{i}^{F}(z)\right)\right)}{\prod_{i \in \mathrm{I}} 2 i \sin \left(\pi\left(Q_{i}(u)+Q_{i}^{F}(z)\right)\right)} \sum_{k \in \mathrm{K}}(-1)^{r_{k}} \mathrm{e}^{2 \pi i\left(\mathrm{q}_{k}(u)+\mathrm{q}_{k}^{F}(z)\right)} \\
& =\prod_{\alpha}\left(x^{-\frac{\alpha}{2}}-x^{\frac{\alpha}{2}}\right) \frac{\prod_{j \in \mathrm{J}}\left(x^{-\frac{q_{j}}{2}} y^{-\frac{q_{j}^{F}}{2}}-x^{\frac{q_{j}}{2}} y^{\frac{q_{j}^{F}}{2}}\right)}{\prod_{i \in \mathrm{I}}\left(x^{\frac{Q_{i}}{2}} y^{\frac{Q_{i}^{F}}{2}}-x^{-\frac{Q_{i}}{2}} y^{-\frac{Q_{i}^{F}}{2}}\right)} \sum_{k \in \mathrm{K}}(-1)^{r_{k}} x^{\mathrm{q}_{k}} y^{\mathrm{q}_{k}^{F}}
\end{aligned}
$$

with $x=\mathrm{e}^{2 \pi i u}$ and $y=\mathrm{e}^{2 \pi i z}$. The second line is easy to remember, as it carries a clear meaning in the operator formalism, and also will be useful for the actual computation.

An $\mathcal{N}=4$ theory is a special case, with a chiral multiplet in the representation $\mathfrak{g}_{\mathbf{C}} \oplus V$, a fermi multiplet in the representation $V$ and no Wilson line. For an effectively compact $\mathcal{N}=4$ theory, we only have to consider the canonical $\mathbf{y}^{2 \boldsymbol{J}_{-}}$twist, with the charges as in (2.50). We shall often write $\mathbf{y}=\mathrm{e}^{\pi i \mathbf{z}}$ so that the twist is by $\mathrm{e}^{2 \pi i \mathbf{z} \boldsymbol{J}_{-}}$(note the difference from $y=\mathrm{e}^{2 \pi i z}$. If we choose $(-1)^{F}=(-1)^{2 J_{3}}$ where $J_{3}$ is an $\mathrm{SU}(2)$ R-symmetry generator, then, we need to set $C_{\ell}=(-1)^{\ell}$ which yields

$$
\begin{aligned}
g & =\left(\frac{1}{2 i \sin (\pi \mathbf{z})}\right)^{\ell} \prod_{\alpha} \frac{\sin (-\pi \alpha(u))}{\sin (\pi(\alpha(u)-\mathbf{z}))} \prod_{i \in \mathrm{I}} \frac{\sin \left(-\pi\left(Q_{i}(u)+\left(\frac{R_{i}}{2}-1\right) \mathbf{z}\right)\right)}{\sin \left(\pi\left(Q_{i}(u)+\frac{R_{i}}{2} \mathbf{z}\right)\right)} \\
& =\left(\frac{1}{\mathbf{y}-\mathbf{y}^{-1}}\right)^{\ell} \prod_{\alpha} \frac{x^{-\frac{\alpha}{2}}-x^{\frac{\alpha}{2}}}{x^{\frac{\alpha}{2}} \mathbf{y}^{-1}-x^{-\frac{\alpha}{2}} \mathbf{y}} \prod_{i \in \mathrm{I}} \frac{x^{-\frac{Q_{i}}{2}} \mathbf{y}^{-\left(\frac{R_{i}}{2}-1\right)}-x^{\frac{Q_{i}}{2}} \mathbf{y}^{\frac{R_{i}}{2}-1}}{x^{\frac{Q_{i}}{2}} \mathbf{y}^{\frac{R_{i}}{2}}-x^{-\frac{Q_{i}}{2}} \mathbf{y}^{-\frac{R_{i}}{2}}}
\end{aligned}
$$

where $\left(Q_{i}, R_{i}\right)$ are the $T$-weights and $\mathrm{U}(1) \mathrm{R}$-charges of $V$. If the theory is not effectively compact, we need to include an $\mathcal{N}=4$ flavor twist. The effect is to make the replacement

$$
x^{ \pm \frac{Q_{i}}{2}} \longrightarrow x^{ \pm \frac{Q_{i}}{2}} y^{ \pm \frac{Q_{i}^{F}}{2}}
$$

in the last factors of (4.97), where $y$ is the parameter of flavor twist and $Q_{i}^{F}$ are the flavor weights of $V$. 


\section{Remarks.}

1. The above result is valid under the condition that $\zeta$ is $\mathscr{I}_{S}$-generic. When the gauge group is Abelian, this is always the case as long as $\zeta$ is in the interior of a phase. However, this is not always the case in non-Abelian gauge theory. A counter example is found in the triangle quiver with rank vector $(k, 1,1)$ : the III-IV wall in figure 4 is in the interior of a phase when the condition (2.90) is satisfied, but the FI parameter there is not $\mathscr{I}_{S}$-generic for any $(a, b, c)$. If $\zeta$ is at such a point, we do not have a formula, while the index is well-defined and is expected to be the same as the one in the neighboring point. In the next section, we show that the result does not change if $\zeta$ goes across such a point, when the non-genericity is of a simple type.

2. The result (4.94) can be written as

$$
I=\frac{1}{|W|} \sum_{p} \underset{p}{\operatorname{JK}-\operatorname{Res}}(Q(p), \zeta)\left[g \mathrm{~d}^{\ell} u\right]
$$

where $p$ runs over all isolated intersections of singular hyperplanes and $Q(p)$ is the set of charges defining the singular hyperplanes that meet at $p$. This way of writing allows us to generalize the statement to the case with degenerate intersections of hyperplanes, i.e., points $p$ with $|Q(p)|>\ell$. This generalization is a proposal that remains to be justified. Recall that the JK residue is defined $[44,45]$ by the property

$$
\underset{p}{\operatorname{JK}-\operatorname{Res}}(Q(p), \zeta)\left[\frac{\mathrm{d}^{\ell} u}{Q_{1}(u) \cdots Q_{\ell}(u)}\right]= \begin{cases}\frac{(2 \pi i)^{\ell}}{\left|\operatorname{det}\left(Q_{1} \cdots Q_{\ell}\right)\right|} & \text { if } \zeta \in \mathcal{C}_{Q_{1} \cdots Q_{\ell}} \\ 0 & \text { otherwise }\end{cases}
$$

for linearly independent elements $Q_{1}, \ldots, Q_{\ell}$ of $Q(p)$. Note that dependence on $\zeta$ is only via the chamber (of $i \mathrm{t}^{*}$ ) with respect to $Q(p)$ to which $\zeta$ belongs. In fact, it is a function of a chamber, not of a particular element of $i t^{*}$ like $\zeta$, in the original definition $[44,45]$ : the condition " $\zeta \in \mathcal{C}_{Q_{1} \cdots Q_{\ell}}$ " in (4.100) is replaced by "the chamber $\subset \mathcal{C}_{Q_{1} \cdots Q_{\ell}}$ ".

\section{A wall crossing formula}

When the FI parameter $\zeta$ goes from one phase to another through a phase boundary, the index may jump as some of the ground states may run away to infinity of the Coulomb branch or of the Coulomb directions of mixed branches. Our index computation is consistent with this picture - the change of the index can be expressed as the integral along a cycle at infinity of the moduli space $\mathfrak{M}$. We have seen this explicitly in the analysis of $\mathrm{U}(1)$ theories, see (4.41) in section 4.3. For a general theory, this can be seen by keeping $\eta$ to be the FI parameter before the wall crossing. Then, the integrals at infinity (4.86), which are all zero before the wall crossing, may become non-zero after the wall crossing, while the residues in the bulk of $\mathfrak{M}$ do not change as they depend only on $\eta$, not on $\zeta$.

In this section, we evaluate this change of the index and compare the result with the Coulomb branch analysis in section 3 , in the simple wall crossing case: i.e. wall crossing across the phase boundary which supports a mixed branch of rank 1 . 


\section{$5.1 \quad \mathrm{U}(1)$ theories}

We consider a general $\mathrm{U}(1)$ gauge theory and look at the change of the index as the FI parameter $\zeta$ moves from positive to negative. As we have seen in section 4.3 , the change of the index is given by the integration along the circle at infinity of $\mathfrak{M}=\mathbf{C} / \mathbf{Z}$. In the single valued coordinate $x=\mathrm{e}^{2 \pi i u}$, the boundaries are the big circle at $x=\infty$ and the small circle at $x=0$ and the wall crossing formula is given by

$$
\Delta I=\frac{1}{2 \pi i}\left[\oint_{0}-\oint_{\infty}\right] \frac{\mathrm{d} x}{x} g(x, y)
$$

where $g(x, y)$ is given in (4.96) (or (4.97) for $\mathcal{N}=4$ theories).

Let us first consider a general $\mathcal{N}=4$ theory. The function $g$ given in (4.97) has limits at both ends,

$$
g(x, \mathbf{y}) \longrightarrow \frac{(-1)^{N_{\text {eff }}^{(4)}}}{\mathbf{y}-\mathbf{y}^{-1}} \begin{cases}\mathbf{y}^{-N_{\text {eff }}^{(4)}} & x \rightarrow \infty \\ \mathbf{y}^{N_{\text {eff }}^{(4)}} & x \rightarrow 0\end{cases}
$$

We recall that $N_{\text {eff }}^{(4)}$ is the number of positively charged fields minus the number of negatively charged fields. Therefore, we find

$$
\begin{aligned}
\Delta I & =(-1)^{N_{\mathrm{eff}}^{(4)}} \frac{\mathbf{y}^{N_{\mathrm{eff}}^{(4)}}-\mathbf{y}^{-N_{\mathrm{eff}}^{(4)}}}{\mathbf{y}-\mathbf{y}^{-1}} \\
& =(-1)^{N_{\mathrm{eff}}^{(4)}} \operatorname{sgn}\left(N_{\mathrm{eff}}^{(4)}\right)\left(\mathbf{y}^{\left|N_{\mathrm{eff}}^{(4)}\right|-1}+\cdots+\mathbf{y}^{-\left(\left|N_{\mathrm{eff}}^{(4)}\right|-1\right)}\right) .
\end{aligned}
$$

The answer is the same even in the presence of an $\mathcal{N}=4$ flavor twist (4.98). This result agrees with the wall crossing formula based on the Coulomb branch analysis (3.57).

Let us next consider $\mathcal{N}=2$ theories. As a warm up, we first look at the untwisted index of the $\mathbf{C P}^{N-1}$ model with Wilson line q, for which

$$
g(x)=\frac{x^{\mathrm{q}}}{\left(x^{\frac{1}{2}}-x^{-\frac{1}{2}}\right)^{N}} .
$$

Note that

$$
\begin{gathered}
\mathrm{q} \geq \frac{N}{2}: g(x) \longrightarrow \begin{cases}x^{\mathrm{q}-\frac{N}{2}}\left(1-x^{-1}\right)^{-N} & x \rightarrow \infty, \\
0 & x \rightarrow 0,\end{cases} \\
|\mathrm{q}|<\frac{N}{2}: g(x) \longrightarrow 0 \quad x \rightarrow \infty \text { and } 0, \\
\mathrm{q} \leq-\frac{N}{2}: g(x) \longrightarrow \begin{cases}0 & x \rightarrow \infty, \\
x^{\mathrm{q}+\frac{N}{2}}(x-1)^{-N} & x \rightarrow 0 .\end{cases}
\end{gathered}
$$

Recall that $x$ is related to the zero mode of the scalar component $\sigma$ via (4.9) as

$$
x=\mathrm{e}^{2 \pi i u}=\exp \left(-i \beta v_{\tau}-\beta \sigma\right)
$$


and hence $x \rightarrow \infty$ corresponds to $\sigma \rightarrow-\infty$ while $x \rightarrow 0$ corresponds to $\sigma \rightarrow+\infty$. In view of this, the above behaviour of $g(x)$ is perfectly consistent with the behaviour (3.40)-(3.41) of the wavefunction for the Coulomb branch vacua. The change of the index is

$$
\begin{aligned}
\mathrm{q} & \geq \frac{N}{2}: \Delta I=-\#\left\{\left(\bar{n}_{1}, \ldots, \bar{n}_{N}\right)|| \bar{n} \mid=\mathrm{q}-\frac{N}{2}\right\}, \\
|\mathrm{q}| & <\frac{N}{2}: \Delta I=0, \\
\mathrm{q} & \leq-\frac{N}{2}: \Delta I=(-1)^{N} \#\left\{\left(n_{1}, \ldots, n_{N}\right)|| n \mid=-\mathrm{q}-\frac{N}{2}\right\} .
\end{aligned}
$$

This matches with the Coulomb branch result, $\Delta I=\Delta_{C} I$.

Let us now move on to a general $\mathcal{N}=2$ theory. It is enough to consider one term of (4.96) with the Wilson line charge $\left(\mathrm{q}, \mathrm{q}^{F}\right)$ :

$$
g=x^{\mathrm{q}} y^{\mathrm{q}^{F}} \frac{\prod_{j}\left(x^{-\frac{q_{j}}{2}} y^{-\frac{q_{j}^{F}}{2}}-x^{\frac{q_{j}}{2}} y^{\frac{q_{j}^{F}}{2}}\right)}{\prod_{i}\left(x^{\frac{Q_{i}}{2}} y^{\frac{Q_{i}^{F}}{2}}-x^{-\frac{Q_{i}}{2}} y^{-\frac{Q_{i}^{F}}{2}}\right)} .
$$

Let us look at the behaviour at $x \rightarrow \infty$ which corresponds to $\sigma \rightarrow-\infty$. We expand the denominator factors as $\sum_{\bar{n}_{i}=0}^{\infty}\left(x^{-Q_{i}} y^{-Q_{i}^{F}}\right)^{\bar{n}_{i}+\frac{1}{2}}$ if $Q_{i}>0$ and $(-1) \sum_{n_{i}=0}^{\infty}\left(x^{Q_{i}} y^{Q_{i}^{F}}\right)^{n_{i}+\frac{1}{2}}$ if $Q_{i}<0$. Also, we write the numerator factors as $\sum_{m_{j}=0,1}(-1)^{m_{j}}\left(x^{q_{j}} y^{q_{j}^{F}}\right)^{m_{j}-\frac{1}{2}}$. Then, the residue is picked for each $\left(\bar{n}_{i}, n_{i}, m_{j}\right)$ solving

$$
\mathrm{q}+\sum_{j} q_{j}\left(m_{j}-\frac{1}{2}\right)-\sum_{Q_{i}>0} Q_{i}\left(\bar{n}_{i}+\frac{1}{2}\right)+\sum_{Q_{i}<0} Q_{i}\left(n_{i}+\frac{1}{2}\right)=0,
$$

with the result

$$
-(-1)^{\#\left\{j \mid m_{j}=1\right\}}(-1)^{\#\left\{i \mid Q_{i}<0\right\}} y^{\mathrm{q}^{F}+\sum_{j} q_{j}^{F}\left(m_{j}-\frac{1}{2}\right)-\sum_{Q_{i}>0} Q_{i}^{F}\left(\bar{n}_{i}+\frac{1}{2}\right)+\sum_{Q_{i}<0} Q_{i}^{F}\left(n_{i}+\frac{1}{2}\right)} .
$$

The equation (5.13) is nothing but the condition (3.47) for the Coulomb branch vacuum on $\sigma<0$, and the residue (5.14) is equal to $-(-1)^{|m|} y^{Q^{F}}$ for $Q^{F}$ given in (3.54) and $(-1)^{|m|}=$ $(-1)^{\sum_{j} m_{j}+\sum_{i} m_{i}}$ in which $m_{i}=0$ for $Q_{i}>0$ and $m_{i}=1$ for $Q_{i}<0$. In particular, it is equal to the $\sigma<0$ contribution to the result (3.56). The analysis for $x \rightarrow 0$ is similar and matches with the $\sigma>0$ contribution to the result (3.56). Thus we conclude $\Delta I=\Delta_{C} I$.

Using this agreement in a general $\mathrm{U}(1)$ gauge theory, we can check that $\Delta I$ matches with the mixed branch result in section 3.4 for a simple wall crossing in higher rank theories. We separate the discussion to Abelian and non-Abelian theories.

\subsection{General Abelian theory}

In a general Abelian theory, any phase boundary $\mathcal{W}$ is simple, with a mixed branch with unbroken gauge group $G_{1} \subset G$ isomorphic to $\mathrm{U}(1)$. It is a codimension one cone spanned 
by $(\ell-1)$ charges, or an open cone therein. We would like to compute the change of the index when $\zeta$ goes through an interior of the wall $\mathcal{W}$.

There are two ways to find it: (i) see what happens to the derivation of the formula (4.91), and (ii) compare the results (4.91) for the two sides of the wall. Both can be done, but we choose to do (ii) as it is easier to present.

The result (4.91) is the sum of $I_{i_{1} \ldots i_{\ell}}$ over $\left\{i_{1}, \ldots, i_{\ell}\right\}$ such that $\zeta$ is inside the cone $\mathcal{C}_{Q_{i_{1}} \ldots Q_{i_{\ell}}}$ spanned by $Q_{i_{1}}, \ldots, Q_{i_{\ell}}$. Therefore, the contribution $I_{i_{1} \ldots i_{\ell}}$ disappears or appears when $\zeta$ goes out of or comes into the cone $\mathcal{C}_{Q_{i_{1}} \ldots Q_{i_{\ell}}}$, respectively. The boundary of $\mathcal{C}_{Q_{i_{1}} \ldots Q_{i_{\ell}}}$ consists of the cones spanned by $(\ell-1)$ elements of $\left\{Q_{i_{1}}, \ldots, Q_{i_{\ell}}\right\}$. Therefore, there is a non-trivial contribution to the change $\Delta I$ of the index when that cone includes the wall $\mathcal{W}$ under consideration. This leads to the following formula.

Before writing down the formula, let us introduce some notation. Let $\mathcal{H}_{\mathcal{W}}^{+}$and $\mathcal{H}_{\mathcal{W}}^{-}$be the two halves of $i t^{*}$ separated by the hyperplane $\mathbf{R} \mathcal{W}$ that includes the wall $\mathcal{W}$, to which $\zeta$ belongs before and after the wall crossing. Suppose $\mathcal{W}$ is included as an open subset of the cone $\mathcal{C}_{Q_{1}, \ldots, Q_{\ell-1}}$. Then $\zeta$ before (resp. after) the wall crossing is included in the cone $\mathcal{C}_{Q_{i}, \ldots, Q_{\ell-1}, Q_{i_{\ell}}}$ if and only if $Q_{i_{\ell}} \in \mathcal{H}_{\mathcal{W}}^{+}\left(\right.$resp. $\left.Q_{i_{\ell}} \in \mathcal{H}_{\mathcal{W}}^{-}\right)$. Therefore, the change of the index is

$$
\Delta I=\sum_{\mathcal{W} \subset \mathcal{C}_{Q_{i_{1}}, \ldots, Q_{i_{\ell-1}}}}\left(-\sum_{Q_{i_{\ell} \in \mathcal{H}_{\mathcal{W}}^{+}}} I_{i_{1} \ldots i_{\ell}}+\sum_{Q_{i_{\ell} \in \mathcal{H}_{\mathcal{W}}^{-}}} I_{i_{1} \ldots i_{\ell}}\right) .
$$

This formula can be simplified. Recall that

$$
I_{i_{1} \ldots i_{\ell}}=\epsilon_{i_{1} \ldots i_{\ell}} \int_{S_{i_{1} \ldots i_{\ell}}} \mathrm{d}^{\ell} u g(u, z),
$$

where $\epsilon_{i_{1} \ldots i_{\ell}}$ is a sign defined by (4.93). The orientation of $\Gamma_{i_{1} \ldots i_{\ell}}$ is opposite between the one with $Q_{i_{\ell}} \in \mathcal{H}_{\mathcal{W}}^{+}$and the one with $Q_{i_{\ell}} \in \mathcal{H}_{\mathcal{W}}^{-}$. Note also that the integrand of (4.93) does not depend on $Q_{i_{\ell}}$ as long as it is linearly independent of $Q_{i_{1}}, \ldots, Q_{i_{\ell-1}}$. So, we may write $\epsilon_{i_{1} \ldots i_{\ell}}= \pm \epsilon_{i_{1} \ldots i_{\ell-1} \infty}^{+}$for $Q_{i_{\ell}} \in \mathcal{H}_{\mathcal{W}}^{ \pm}$. Then, inside the big parenthesis of (5.15) can be written as

$$
-\sum_{i_{\ell} \in \mathscr{I}_{S}} \epsilon_{i_{1} \ldots i_{\ell-1} \infty}^{+} \int_{S_{i_{1} \ldots i_{\ell-1} i_{\ell}}} \mathrm{d}^{\ell} u g(u, z)
$$

Using (4.64), this is equal to

$$
\epsilon_{i_{1} \ldots i_{\ell-1} \infty}^{+} \int_{S_{i_{1} \ldots i_{\ell-1} \infty}} \mathrm{d}^{\ell} u g(u, z)=: I_{i_{1} \ldots i_{\ell-1} \infty}^{+} .
$$

Therefore, the wall crossing formula (5.15) can be written as

$$
\Delta I=\sum_{\mathcal{W} \subset \mathcal{C}_{Q_{i_{1}}, \ldots, Q_{i_{\ell-1}}}} I_{i_{1} \ldots i_{\ell-1} \infty}^{+} .
$$

This can be processed further. Recall from section 3.4 that the effective theory on the mixed branch at the wall $\mathcal{W}$ has two sectors - $(\mathrm{C})$ with gauge group $G_{1}$ and $(\mathrm{H})$ with gauge group $H=C\left(G_{1}\right) / G_{1}$ which is $T / G_{1}$ when $G(=T)$ is Abelian. Matters in (H) are those 
with charges in the hyperplane $\mathbf{R} \mathcal{W}$ and everything else belongs to $(\mathrm{C})$. Note that $Q \in i \mathrm{t}^{*}$ belongs to $\mathbf{R} \mathcal{W}$ if and only if it vanishes on $\mathfrak{g}_{1}$. We denote the projection $\mathfrak{t} \rightarrow \mathfrak{t} / \mathfrak{g}_{1}=\mathfrak{h}$ by $u \mapsto u_{\mathfrak{h}}$. We also choose an element $Q_{\infty} \in i \mathfrak{t}^{*} \backslash \mathbf{R} \mathcal{W}$ such that $\mathrm{e}^{2 \pi i \xi} \rightarrow \mathrm{e}^{2 \pi i Q_{\infty}(\xi)}$ defines an isomorphism $G_{1} \subset T \rightarrow \mathrm{U}(1)$. Then, we have an isomorphism

$$
\begin{aligned}
S_{i_{1} \ldots i_{\ell-1} \infty}: & =\left\{u \in \mathfrak{t}_{\mathbf{C}} / Q^{\vee} \mid \begin{array}{l}
\left|Q_{i_{a}}(u)-c_{i_{a}}\right|=\varepsilon, a=1, \ldots, \ell-1 \\
\operatorname{Im} Q_{\infty}(u)= \pm R
\end{array}\right\} \\
\cong & \quad \times\left\{u_{\mathfrak{h}} \in \mathfrak{h}_{\mathbf{C}} / \mathrm{Q}_{H}^{\vee}\left|Q_{i_{a}}(u)-c_{i_{a}}\right|=\varepsilon, a=1, \ldots, \ell-1\right\} \\
\quad & \left.Q_{\infty}(u) \in \mathbf{C} / \mathbf{Z} \mid \operatorname{Im} Q_{\infty}(u)= \pm R\right\} \\
= & S_{i_{1} \ldots i_{\ell-1}}^{(\mathrm{H})} \times S_{\infty}^{(\mathrm{C})} .
\end{aligned}
$$

This holds including the orientation provided $\epsilon_{i_{1} \ldots i_{\ell-1} \infty}^{+}=\epsilon_{i_{1} \ldots i_{\ell-1}}^{(\mathrm{H})}$. The integral (5.18) can then be written as

$$
I_{i_{1} \ldots i_{\ell-1} \infty}^{+}=\epsilon_{i_{1} \ldots i_{\ell-1}}^{(\mathrm{H})} \int_{S_{i_{1} \ldots i_{\ell-1}}^{(\mathrm{H})}} \int_{S_{\infty}^{(\mathrm{C})}} \mathrm{d}^{\ell} u g(u, z) .
$$

We may write $g(u, z)=g^{(\mathrm{H})}\left(u_{\mathfrak{h}}, z\right) \cdot g^{(\mathrm{C})}(u, z)$, where $g^{(\mathrm{H})}\left(u_{\mathfrak{h}}, z\right)$ is the $g$-function for the theory $(\mathrm{H})$ and $g^{(\mathrm{C})}(u, z)$ is everything else - we put all Wilson line factors into the latter even if some of the charges may vanish on $\mathfrak{g}_{1}$. The integration of $g^{(\mathrm{C})}(u, z)$ along $S_{\infty}^{(\mathrm{C})}$ yields the change of the index in the theory $(\mathrm{C})$,

$$
\int_{S_{\infty}^{(\mathrm{C})}} \mathrm{d}^{\ell} u g^{(\mathrm{C})}(u, z)=\mathrm{d}^{\ell-1} u_{\mathfrak{h}} \Delta I^{(\mathrm{C})}\left(u_{\mathfrak{h}}, z\right) .
$$

Note that the result may depend on $u_{\mathfrak{h}}$ and provides background Wilson lines for the theory (H). Therefore, we have

$$
\Delta I=\sum_{\zeta_{0} \in \mathcal{C}_{Q_{i_{1}} \ldots Q_{i_{\ell-1}}^{(\mathrm{H})}}} \epsilon_{i_{1} \ldots i_{\ell-1}}^{(\mathrm{H})} \int_{S_{i_{1} \ldots i_{\ell-1}}^{(\mathrm{H})}} \mathrm{d}^{\ell-1} u_{\mathfrak{h}} g^{(\mathrm{H})}\left(u_{\mathfrak{h}}, z\right) \Delta I^{(\mathrm{C})}\left(u_{\mathfrak{h}}, z\right)
$$

Here we used the fact that $\mathcal{W} \subset \mathcal{C}_{Q_{i_{1}} \ldots Q_{i_{\ell-1}}}$ is equivalent to $\zeta_{0} \in \mathcal{C}_{Q_{i_{1}} \ldots Q_{i_{\ell-1}}}^{(\mathrm{H})}$ where $\zeta_{0}$ is a point on the wall $\mathcal{W}$ regarded as an element of $i \mathfrak{h}^{*}$. This yields the index for the theory (H) with the FI parameter $\zeta_{0}$. The result may be written as

$$
\Delta I=\left(I_{\zeta_{0}}^{(\mathrm{H})} * \Delta I^{(\mathrm{C})}\right)(z)
$$

Since we have shown $\Delta I=\Delta_{C} I$ for $\mathrm{U}(1)$ theories, we find that the above agrees with the mixed branch result (3.60).

\subsection{Simple wall crossing in a non-Abelian theory}

Let us now discuss the simple wall crossing in a general non-Abelian gauge theory. Before starting, we describe the set up and introduce some notations and terminology. The space 
of FI parameters $i\left(\mathfrak{t}^{*}\right)^{W}$ can be regarded as a subspace of $i \mathfrak{t}^{*}$. A general phase boundary is a codimension one cone in $i\left(\mathfrak{t}^{*}\right)^{W}$, and it is the intersection of $i\left(\mathfrak{t}^{*}\right)^{W}$ with cones in $i \mathfrak{t}^{*}$ spanned by $(\ell-1)$ or less charges. A simple phase boundary $\mathcal{W}$ has the following special property: it is the intersection of $i\left(\mathfrak{t}^{*}\right)^{W}$ with cones spanned by exactly $(\ell-1)$ charges, and the hyperplanes spanned by such charges are Weyl images of one another. The unbroken subgroup $G_{1}$ may be defined as the subgroup of $T$ whose Lie algebra $\mathfrak{g}_{1}$ is the orthogonal to one of such hyperplanes. (Note that $G_{1} \subset G$ is defined only up to conjugation, and other conjugation images in $T$ are the orthogonal to the other hyperplanes.) The effective theory on the mixed branch at the wall $\mathcal{W}$ has two sectors - (C) with gauge group $G_{1}$ and (H) with gauge group $H=C\left(G_{1}\right) / G_{1}$. Let us collect some useful facts on $G_{1}$ and $H$ :

(i) $T / G_{1}=: T_{H}$ is a maximal torus of $H$.

(ii) The normalizer of $T_{H}$ in $H$ is $\left(N_{T} \cap C\left(G_{1}\right)\right) / G_{1}$ and hence the Weyl group of $H$ is $W_{H}=\left[\left(N_{T} \cap C\left(G_{1}\right)\right) / G_{1}\right] /\left[T / G_{1}\right] \cong\left(N_{T} \cap C\left(G_{1}\right)\right) / T$.

(iii) In particular, $W_{H}$ may be regarded as a subgroup of $W$ consisting of elements that does not move $\mathfrak{g}_{1} \subset \mathfrak{t}$.

(iv) Therefore, $|W| /\left|W_{H}\right|=\left|W / W_{H}\right|$ is an integer, and that is the number of hyperplanes that meets $i\left(\mathfrak{t}^{*}\right)^{W}$ at the wall $\mathcal{W}$.

By (i), we may regard the hyperplane $\mathfrak{g}_{1}^{\perp} \subset \mathfrak{t}^{*}$ as the dual of a Cartan subalgebra of $H, \mathfrak{g}_{1}^{\perp} \cong$ $\mathfrak{t}_{H}^{*}$. Matters in the theory $(\mathrm{H})$ consists of those whose charges belong to this hyperplane $i \mathfrak{g}_{1}^{\perp}$, and everything else belongs to (C). We denote the projection $\mathfrak{t} \rightarrow \mathfrak{t} / \mathfrak{g}_{1} \cong \mathfrak{t}_{H}$ by $u \mapsto u_{\mathfrak{h}}$.

Let us now evaluate the change of the index. Let $\mathcal{H}_{\mathfrak{g}_{1}^{\perp}}^{+}$and $\mathcal{H}_{\mathfrak{g}_{1}^{\perp}}^{-}$be the two halves of $i \mathfrak{t}^{*}$ separated by the hyperplane $i \mathfrak{g}_{1}^{\perp}$ to which $\zeta$ belongs before and after the wall crossing. Suppose the wall $\mathcal{W}$ is included as an open subset of the cone $\mathcal{C}_{Q_{1}, \ldots, Q_{\ell-1}}$ in $i \mathfrak{g}_{1}^{\perp}$. Then $\zeta$ before (resp. after) the wall crossing is included in the cone $\mathcal{C}_{Q_{i}, \ldots, Q_{\ell-1}, Q_{i_{\ell}}}$ if and only if $Q_{i_{\ell}} \in \mathcal{H}_{\mathfrak{g}_{1}^{\perp}}^{+}\left(\right.$resp. $\left.Q_{i_{\ell}} \in \mathcal{H}_{\mathfrak{g}_{1}^{\perp}}^{-}\right)$. Using this as well as the fact (iv) above, we find that the change of the index is given by

$$
\begin{aligned}
& \Delta I=\frac{1}{|W|}\left|\frac{W}{W_{H}}\right| \sum_{\mathcal{W} \subset \mathcal{C}_{Q_{i_{1}}, \ldots, Q_{i_{\ell-1}}} \subset i_{\mathfrak{g}_{1}^{\perp}}}\left(-\sum_{Q_{i_{\ell} \in \mathcal{H}_{\mathfrak{g}_{\perp}}^{+}}} I_{i_{1} \ldots i_{\ell}}+\sum_{Q_{i_{\ell} \in \mathcal{H}_{\mathfrak{g}_{\perp}}^{-}}^{-}} I_{i_{1} \ldots i_{\ell}}\right) \\
& =\frac{1}{\left|W_{H}\right|} \sum_{\mathcal{W} \subset \mathcal{C}_{Q_{i_{1}}, \ldots, Q_{i_{\ell-1}}} \subset i_{1}^{\perp}} I_{i_{1} \ldots i_{\ell-1} \infty}^{+} .
\end{aligned}
$$

The definition of $I_{i_{1} \ldots i_{\ell-1} \infty}^{+}$and the process of going to the second line is exactly the same as in the Abelian case. This can be processed further, again as in the Abelian theories, based on the factorization

$$
S_{i_{1} \ldots i_{\ell-1} \infty} \cong S_{i_{1} \ldots i_{\ell-1}}^{(\mathrm{H})} \times S_{\infty}^{(\mathrm{C})}
$$

as in (5.20), with $\epsilon_{i_{1} \ldots i_{\ell-1} \infty}^{+}=\epsilon_{i_{1} \ldots i_{\ell-1}}^{(\mathrm{H})}$, and the factorization

$$
g(u, z)=g^{(\mathrm{H})}\left(u_{\mathfrak{h}}, z\right) g^{(\mathrm{C})}(u, z) .
$$


In the latter, the root factor of $g(u, z)$ in (4.96) and (4.97) should be included in $g^{(\mathrm{H})}$ if $\alpha \in i \mathfrak{g}_{1}^{\perp}$ (i.e., if it is a root of $C\left(G_{1}\right) / G_{1}=H$ ) and in $g^{(\mathrm{C})}$ if $\alpha$ is non-zero on $\mathfrak{g}_{1}$ (i.e., if $\mathfrak{g}_{\alpha}$ do not commute with $\left.\mathfrak{g}_{1}\right)$. This yields

$$
\begin{aligned}
& \Delta I=\frac{1}{\left|W_{H}\right|} \sum_{\zeta_{0} \in \mathcal{C}_{Q_{i_{1}} \ldots Q_{i_{\ell-1}}}^{(\mathrm{H})}} \epsilon_{i_{1} \ldots i_{\ell-1}}^{(\mathrm{H})} \int_{S_{i_{1} \ldots i_{\ell-1}}^{(\mathrm{H})}} \mathrm{d}^{\ell-1} u_{\mathfrak{h}} g^{(\mathrm{H})}\left(u_{\mathfrak{h}}, z\right) \Delta I^{(\mathrm{C})}\left(u_{\mathfrak{h}}, z\right) \\
& =\left(I^{(\mathrm{H})} * \Delta I^{(\mathrm{C})}\right)(z) \text {. }
\end{aligned}
$$

Since we have shown $\Delta I=\Delta_{C} I$ for $\mathrm{U}(1)$ theories, we find that the above agrees with the mixed branch result (3.60).

Note that the above computation applies to the case where $\mathcal{W}$ is not a real phase boundary. Recall that a non $\mathscr{I}_{S}$-generic point does not always admit a mixed branch solution, in which case it must be in the interior of a phase. For example, the III-IV wall in figure 4 of the triangle quiver in which the condition (2.90) is satisfied. When $k=2$, it is of simple type, i.e., it is the intersection of $i\left(\mathfrak{t}^{*}\right)^{W}$ with the cone spanned by exactly $\ell-1(=2)$ charges. For such a case, since the mixed branch is empty, the index of theory (H) must vanish, $I_{\zeta_{0}}^{(\mathrm{H})}=0$, and hence the index does not jump across $\mathcal{W}, \Delta I=0$.

\section{$\mathcal{N}=4$ theories}

For $\mathcal{N}=4$ theories, we have seen that $\Delta I$ is independent of the flavor twist in the $\mathrm{U}(1)$ theories. Therefore, in either Abelian or non-Abelian theory, $\Delta I^{(\mathrm{C})}\left(u_{\mathfrak{h}}, z\right)$ depends only on the $\boldsymbol{J}_{-}$part of $z$ (denoted by $\mathbf{z}$ ),

$$
\Delta I^{(\mathrm{C})}\left(u_{\mathfrak{h}}, z\right)=\Delta I^{(\mathrm{C})}(\mathbf{z}) .
$$

Therefore, the wall crossing formula has a factorized form,

$$
\Delta I(z)=I^{(\mathrm{H})}\left(\mathbf{z}, z_{\text {other }}\right) \times \Delta I^{(\mathrm{C})}(\mathbf{z}),
$$

in agreement with the mixed branch analysis (3.58).

\section{Systems with $\mathcal{N}=4$ supersymmetry}

In this section, we illustrate the index formula and wall crossing in a few examples with $\mathcal{N}=4$ supersymmetry.

\subsection{Grassmannian}

The first example is the $\mathrm{U}(k)$ gauge theory with $N$ fundamental chiral multiplets. We set $\zeta(D)=r \cdot \operatorname{tr}(D)$. When $N \geq k, r>0$ is the geometric phase with the Grassmannian $G(k, N)$ of $k$ planes in $\mathbf{C}^{N}$ as the target space. For $r<0$ or for $N<k$, supersymmetry is broken.

We shall compute the index in the geometric phase. Since the model is compact, dependence of the flavor twist is trivial and any assignment of R-charge yields the same result. 
For simplicity, we set $R_{i}=0$ for all the chiral matters, for which the $g$ function (4.97) reads

$$
\begin{aligned}
g & =\left(\frac{1}{\mathbf{y}-\mathbf{y}^{-1}}\right)^{k} \prod_{a \neq b} \frac{x_{a}^{-\frac{1}{2}} x_{b}^{\frac{1}{2}}-x_{a}^{\frac{1}{2}} x_{b}^{-\frac{1}{2}}}{x_{b}^{-\frac{1}{2}} \mathbf{y}^{-1}-x_{a}^{-\frac{1}{2}} x_{b}^{\frac{1}{2}} \mathbf{y}}\left(\prod_{a=1}^{k} \frac{x_{a}^{-\frac{1}{2}} \mathbf{y}-x_{a}^{\frac{1}{2}} \mathbf{y}^{-1}}{x_{a}^{\frac{1}{2}}-x_{a}^{-\frac{1}{2}}}\right)^{N} \\
& =(-1)^{k(N-k)} \frac{\mathbf{y}^{-k(N-k)}}{\left(1-\mathbf{y}^{2}\right)^{k}} \frac{\prod_{a \neq b}\left(x_{a}-x_{b}\right)}{\prod_{a \neq b}\left(x_{a}-\mathbf{y}^{2} x_{b}\right)} \frac{\prod_{a=1}^{k}\left(x_{a}-\mathbf{y}^{2}\right)^{N}}{\prod_{a=1}^{k}\left(x_{a}-1\right)^{N}} .
\end{aligned}
$$

Singular hyperplanes are $H_{a}=\left(x_{a}=1\right)$ from the chiral multiplets with the charge vector $e_{a}$ and $H_{a, b}=\left(x_{a}=\mathbf{y}^{2} x_{b}\right)$ from the root $e_{a}-e_{b}$ component of the vector multiplet. Here, $e_{a}$ are the standard basis elements of $i \mathbf{t}^{*} \cong \mathbf{R}^{k}$. Note that

$$
\zeta=r\left(e_{1}+\cdots+e_{k}\right), \quad r>0
$$

The isolated intersection $H_{1} \cap \cdots \cap H_{k}$ should be taken in the JK residue, as $\zeta$ is a positive span of the defining charges $Q=\left\{e_{1}, \ldots, e_{k}\right\}$. What about those involving $H_{a, b}$ 's? Consider for example, $H_{1} \cap \cdots \cap H_{k-1} \cap H_{k, a}$ with $Q=\left\{e_{1}, \ldots, e_{k-1}, e_{k}-e_{a}\right\}$ and $H_{1} \cap \cdots \cap H_{k-1} \cap H_{a, k}$ with $Q=\left\{e_{1}, \ldots, e_{k-1}, e_{a}-e_{k}\right\}$. The former satisfy the condition that $\zeta$ is in the positive span of $Q$, while the latter fails. On the other hand, for the former, the residue vanishes because of the factor $\left(x_{k}-\mathbf{y}^{2}\right)^{N}$ in the numerator in (6.1). Similar reasoning applies to intersections involving more than one vector singularities, because in order to have co-dimension $k$ singularity at least on singularity from the fundamental chiral must be involved.

Thus the only pole that contributes comes from $H_{1} \cap \cdots \cap H_{k}$,

$$
I=\frac{1}{k !} \cdot \frac{1}{(2 \pi i)^{k}} \int_{\left|x_{1}-1\right|=\cdots=\left|x_{k}-1\right|=\varepsilon} \frac{\mathrm{d} x_{1} \cdots \mathrm{d} x_{k}}{x_{1} \cdots x_{k}} g(x, \mathbf{y}) .
$$

At this moment, we do not have a systematic way to carry out the computation. Instead, we can check in examples that it reproduces the known formula [22, 24],

$$
I=(-1)^{k(N-k)} \cdot \frac{\prod_{i=1}^{N}\left(\mathbf{y}^{-i}-\mathbf{y}^{i}\right)}{\prod_{j=1}^{k}\left(\mathbf{y}^{-j}-\mathbf{y}^{j}\right) \prod_{l=1}^{N-k}\left(\mathbf{y}^{-l}-\mathbf{y}^{l}\right)} .
$$

\subsection{Hypersurface in projective space}

Let us next consider Example 2 in section 2.4. $\zeta>0$ is the geometric phase with the degree $d$ hypersurface $X_{f} \subset \mathbf{C P}^{N-1}$, while $\zeta<0$ is the Landau-Ginzburg orbifold $W=$ $f\left(X_{1}, \ldots, X_{N}\right) / \mathbf{Z}_{d}$.

The R-charge can be anything such that the superpotential $W=\operatorname{Pf}(\Phi)$ has charge 2 . We take $R_{P}=2$ and $R_{X}=0$, for which the $g$ function is

$$
\begin{aligned}
g & =\frac{1}{\mathbf{y}-\mathbf{y}^{-1}}\left(\frac{x^{-\frac{1}{2}} \mathbf{y}-x^{\frac{1}{2}} \mathbf{y}^{-1}}{x^{\frac{1}{2}}-x^{-\frac{1}{2}}}\right)^{N} \frac{x^{\frac{d}{2}}-x^{-\frac{d}{2}}}{x^{-\frac{d}{2}} \mathbf{y}-x^{\frac{d}{2}} \mathbf{y}^{-1}} \\
& =(-1)^{N-1} \frac{\mathbf{y}^{1-N}}{\mathbf{y}-\mathbf{y}^{-1}}\left(\frac{x-\mathbf{y}^{2}}{x-1}\right)^{N} \frac{x^{d}-1}{x^{d}-\mathbf{y}^{2}} .
\end{aligned}
$$


In the geometric phase $\zeta>0$, we select the $N$-th order pole at $x=1$ :

$$
\begin{aligned}
I & =\frac{1}{2 \pi i} \oint_{1} \frac{\mathrm{d} x}{x} g(x, \mathbf{y}) \\
& =\left.(-1)^{N-1} \frac{\mathbf{y}^{1-N}}{\mathbf{y}-\mathbf{y}^{-1}} \frac{1}{(N-1) !} \frac{\mathrm{d}^{N-1}}{\mathrm{~d} x^{N-1}}\left[\frac{\left(x-\mathbf{y}^{2}\right)^{N}\left(x^{d}-1\right)}{x\left(x^{d}-\mathbf{y}^{2}\right)}\right]\right|_{x=1} .
\end{aligned}
$$

For example,

$$
\begin{aligned}
I_{N=3}= & \frac{d(d-3)}{2}\left(\mathbf{y}^{-1}+\mathbf{y}\right) \\
I_{N=4}= & \frac{d\left(d^{2}-6 d+11\right)}{6}\left(\mathbf{y}^{-2}+\mathbf{y}^{2}\right)+\frac{d\left(2 d^{2}-6 d+7\right)}{3}, \\
I_{N=5}= & \frac{d\left(d^{3}-10 d^{2}+35 d-50\right)}{24}\left(\mathbf{y}^{-3}+\mathbf{y}^{3}\right)+\frac{d\left(11 d^{3}-50 d^{2}+85 d-70\right)}{24}\left(\mathbf{y}^{-1}+\mathbf{y}\right) \\
& \ldots
\end{aligned}
$$

These are the $\chi_{y}$ genus (2.73) of the hypersurface $X_{f}$. By the Lefschetz hyperplane theorem, the Hodge diamond must be of the form

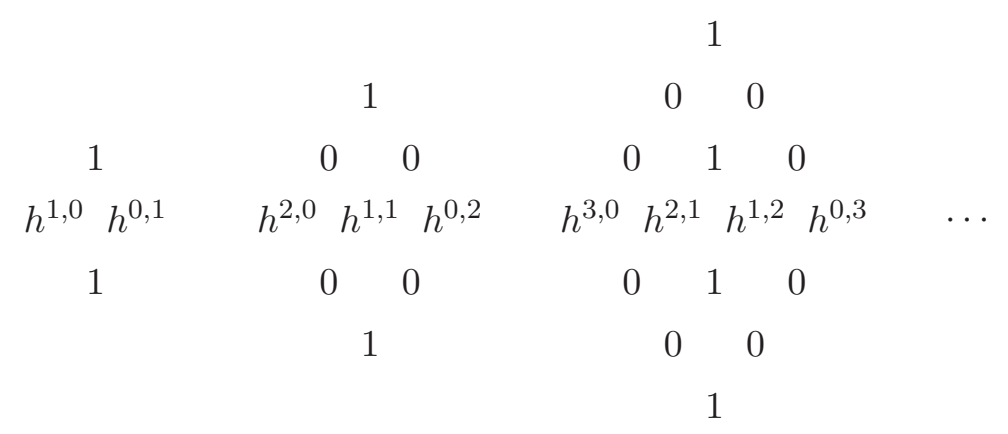

Thus, we can read off the Hodge numbers from the index. For example, for $N=3, X_{f}$ is a curve of genus $h^{1,0}=\frac{d(d-3)}{2}+1$. For $N=4$ it is a surface of $h^{2,0}=\frac{d\left(d^{2}-6 d+11\right)}{6}-1$ and $h^{1,1}=\frac{d\left(2 d^{2}-6 d+7\right)}{3}$. Etc. Check that $h^{N-2,0}=1$ in the Calabi-Yau case $d=N$. Note also that $h^{1,1}=20$ for $d=N=4$ (K3 surface) and $h^{2,1}=101$ for $d=N=5$ (quintic threefold). To find the index in the Landau-Ginzburg phase $\zeta<0$, we use the wall crossing formula (5.3), in which we set $N_{\mathrm{eff}}^{(4)}=N-1$. This yields

$$
I_{\mathrm{LG}}=I_{\text {geometric }}+(-1)^{N-1}\left(\mathbf{y}^{-(N-2)}+\cdots+\mathbf{y}^{N-2}\right)
$$

This is also what we have seen in the Coulomb branch analysis. There we have also seen that the wall crossing states form the spin $j=\frac{\left|N_{\text {eff }}^{(4)}\right|-1}{2}=\frac{N-2}{2}$ representation of the $\mathrm{SU}(2)$ R-symmetry. Since the vertical axis of the Hodge diamond shows the spin $J_{3}$ of the same $\mathrm{SU}(2)$, the 1's in the vertical middle of the Hodge diamond (6.10) drops. This leaves us 
with the following Hodge diamond of the Landau-Ginzburg orbifold:

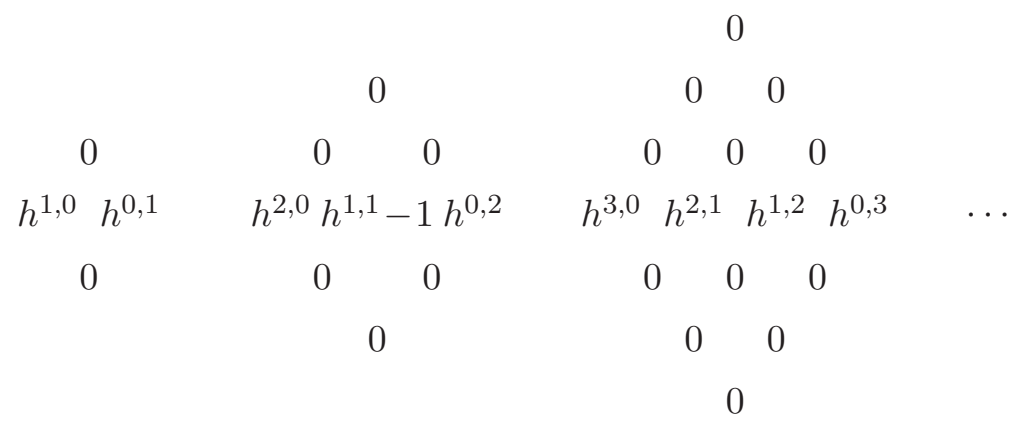

Note that there is a non-trivial wall crossing even in the Calabi-Yau case $d=N$ where $X_{f}$ is a Calabi-Yau manifold. In that case, the elliptic genus of the two-dimensional version of the model is well defined and does not exhibit wall crossing phenomenon, i.e., the Hodge diamond is the same as (6.10) for both the geometric phase and the Landau-Ginzburg orbifold phase. On the other hand, in one-dimension, there is a wall crossing. The difference comes from the twisted sector states, which are present in $2 \mathrm{~d}$ orbifolds but absent in $1 \mathrm{~d}$ orbifolds: in the $2 d$ Landau-Ginzburg orbifold, the ground states from the $d-1(=N-1)$ twisted sectors are nothing but the 1's in the diagonal middle. The horizontal middle of (6.12) are the untwisted sector invariant states, which are present both in $1 \mathrm{~d}$ and $2 \mathrm{~d}$.

When $d \neq N$, the axial $\mathrm{U}(1) \mathrm{R}$-symmetry (which determines the vertical axis of Hodge diamond) is anomalous in $2 \mathrm{~d}$ and the elliptic genus is ill defined. ${ }^{7}$ However, we can still consider the relation between the ground states of the non-linear sigma model and the Landau-Ginzburg orbifold. In $2 \mathrm{~d}$, the two theories are related by the renormalization group. For $d<N$, it is a flow from the sigma model to the Landau-Ginzburg orbifold, and for $d>N$ the flow is in the opposite direction. The spectrum of supersymmetric ground states is continuous along the flow, provided we include the $|N-d|$ isolated massive vacua on the Coulomb branch in the low energy side. This is consistent with the above result on the 1d wall crossing that $(N-1)$ states disappear as we go from the geometric phase to the Landau-Ginzburg orbifold phase. In $2 \mathrm{~d}$, in the same direction, $(N-d)$ states go to Coulomb branch vacua (counted with sign!) and $(d-1)$ states becomes twisted sector states.

\subsection{Flavor decoupling in compact models}

In section 2.2 , we proved that supersymmetric ground states of an effectively compact $\mathcal{N}=4$ theory have charge zero under any flavor symmetry. In particular, the index cannot depend on the flavor twist parameter. In fact, we have already seen this implicitly for the $\mathbf{C P}^{N-1}$ model. That model has $\mathrm{SU}(N)$ flavor symmetry and we may consider the index twisted by that. Since the index vanishes for $\zeta<0$, the index for $\zeta>0$ is determined by the wall crossing part, $I=-\Delta I$, but we have seen that $\Delta I$ is independent of the flavor twist in any $\mathrm{U}(1)$ theory (5.3). ${ }^{8}$ In this subsection, we illustrate the twist independence in

\footnotetext{
${ }^{7}$ The vector U(1) R-symmetry, which determines the horizontal axis of the Hodge diamond, is conserved for any $d$ versus $N$.

${ }^{8}$ In section 8.1 , we shall study $\mathcal{N}=2 \mathbf{C P}^{N-1}$ model, where we will see that the $\mathrm{SU}(N)$ flavor twist dependence of the index is not only non-trivial but carries important information about the ground states.
} 
a more complicated model. We shall see this in the form of independence on the R-charge assignment.

Consider $\mathrm{U}(1)^{k}$ theories with $k+1$ sets of chiral fields of charges, $a_{1}$ number of $(1,0,0, \cdots, 0), a_{2}$ number of $(0,1,0, \cdots, 0), \ldots, a_{k}$ number of $(0,0,0, \cdots, 1)$, and $a_{k+1}$ number of $(1,1,1, \cdots, 1)$. The superpotential is not possible. The classical Higgs branch is present and is compact when all the FI parameters are positive. When some of the FI parameter is negative, supersymmetry is broken. Since there is no superpotential, there is no constraint on the R-charge assignment. For example, we may assign 0 to the first $k$ groups of matter fields, but $q$ to the last group of $a_{k+1}$ fields. Clearly, this is not a part of the gauge symmetry. This gives

$$
\begin{aligned}
g_{a_{1} a_{2} \cdots a_{k} ; a_{k+1}}= & \left(\frac{1}{2 i \sin (\pi \mathbf{z})}\right)^{k} \times \prod_{i=1}^{k}\left(\frac{\sin \left(-\pi\left(u_{i}-\mathbf{z}\right)\right)}{\sin \left(\pi u_{i}\right)}\right)^{a_{i}} \\
& \times\left(\frac{\sin \left(-\pi\left(u_{1}+\cdots+u_{k}+(q-1) \mathbf{z}\right)\right)}{\sin \left(\pi\left(u_{1}+\cdots+u_{k}+q \mathbf{z}\right)\right)}\right)^{a_{k+1}} .
\end{aligned}
$$

We of course look at the phase where all the FI parameters $\zeta_{i}$ are positive. Taking a simultaneous permutations of $a$ 's and $\zeta$ 's, we may further assume that $\zeta_{i \neq k}>\zeta_{k}>0$. There are exactly two relevant poles $u_{*}^{(0)} \equiv\left\{u_{1}=u_{2}=\cdots=u_{k}=0\right\}$ and $u_{*}^{(k)} \equiv\left\{u_{1}=\right.$ $\left.u_{2}=\cdots=u_{k-1}=0=\sum_{i=1}^{k} u_{i}+q \mathbf{z}\right\}$, and the index is

$$
I_{a_{1} \cdots a_{k} ; a_{k+1}}=\operatorname{res}_{u=u_{*}^{(0)}} g_{a_{1} a_{2} \cdots a_{k} ; a_{k+1}}(u)+\operatorname{res}_{u=u_{*}^{(k)}} g_{a_{1} a_{2} \cdots a_{k} ; a_{k+1}}(u) .
$$

Both terms are explicitly dependent on $q$, yet the sum should be independent of $q$.

This $q$-independence is easiest to see when $a_{1}=\cdots=a_{k-1}=1$. Integrating $u_{1}, \ldots, u_{k-1}$ first, we find

$$
I_{1,1, \ldots, 1, a_{k} ; a_{k+1}}=\left[\underset{u_{k}=0}{\operatorname{res}}+\underset{u_{k}=-q \mathbf{z}}{\operatorname{res}}\right] f_{q}^{a_{k} a_{k+1}}\left(u_{k}, \mathbf{z}\right),
$$

where

$$
f_{q}^{a_{k} a_{k+1}}\left(u_{k}, z\right) \equiv \frac{1}{2 i \sin (\pi \mathbf{z})} \cdot\left(\frac{\sin \left(-\pi\left(u_{k}-\mathbf{z}\right)\right)}{\sin \left(\pi u_{k}\right)}\right)^{a_{k}} \cdot\left(\frac{\sin \left(-\pi\left(u_{k}+(q-1) \mathbf{z}\right)\right)}{\sin \left(\pi\left(u_{k}+q \mathbf{z}\right)\right)}\right)^{a_{k+1}} .
$$

The integrand has two other poles, at $e^{2 \pi i u_{k}}=0, \infty$, which are both simple and with which we may trade off the poles $e^{2 \pi i u_{k}}=1, e^{-2 \pi i q z}$. Residues at $e^{2 \pi i u_{k}}=0, \infty$ are manifestly independent of $q$, proving the assertion that the index is independent of $q$. This simple argument generalizes straightforwardly to general $a_{i}$ 's, by turning on most general flavor chemical potentials on the first $k-1$ sets of chiral fields, which reduces all the poles of $u_{i, \ldots, k-1}$ planes to become simple.

We close with a few examples,

$$
\begin{aligned}
& I_{2,4 ; 3}=-\frac{1}{\mathbf{y}^{7}}-\frac{2}{\mathbf{y}^{5}}-\frac{2}{\mathbf{y}^{3}}-\frac{2}{\mathbf{y}}-2 \mathbf{y}-2 \mathbf{y}^{3}-2 \mathbf{y}^{5}-\mathbf{y}^{7}, \\
& I_{4,4 ; 2}=\frac{1}{\mathbf{y}^{8}}+\frac{2}{\mathbf{y}^{6}}+\frac{3}{\mathbf{y}^{4}}+\frac{4}{\mathbf{y}^{2}}+4+4 \mathbf{y}^{2}+3 \mathbf{y}^{4}+2 \mathbf{y}^{6}+\mathbf{y}^{8},
\end{aligned}
$$




$$
I_{3,3,3 ; 3}=-\frac{1}{\mathbf{y}^{9}}-\frac{3}{\mathbf{y}^{7}}-\frac{6}{\mathbf{y}^{5}}-\frac{8}{\mathbf{y}^{3}}-\frac{9}{\mathbf{y}}-9 \mathbf{y}-8 \mathbf{y}^{3}-6 \mathbf{y}^{5}-3 \mathbf{y}^{7}-\mathbf{y}^{9},
$$

which indeed shows no $q$-dependence, showing that all ground states are invariant under the flavor symmetry.

\subsection{Flavor non-decoupling in non-compact models}

When the theory is not effectively compact, the decoupling of flavor twist fails, or rather, flavor twist is necessary to make the index well-defined. The most illustrative example of this is already mentioned in section 4.2 , embedded in the middle of one-loop computation, though with $\mathcal{N}=2$ supersymmetry. Let us consider the $\mathcal{N}=4$ version, that is, the theory (with no gauge group) of a single chiral multiplet on which the $\mathrm{U}(1)$ flavor symmetry acts in the standard way. The Higgs branch is $\mathbf{C}$ and is non-compact. The index with the twist parameter $y$ for the $\mathrm{U}(1)$ is, when we assign R-charge zero,

$$
I=\frac{y^{-\frac{1}{2}} \mathbf{y}-y^{\frac{1}{2}} \mathbf{y}^{-1}}{y^{\frac{1}{2}}-y^{-\frac{1}{2}}} .
$$

Indeed it depends on the twist parameter $y$ and is singular when we turn it off, $y \rightarrow 1$. As stated in section 2.2, Hamiltonian way to understand it is possible only when the real mass $m=m_{3}$ is non-zero, i.e. when $|y|>1$ or $|y|<1$, which makes the theory compact by the Harmonic oscillator potential. Then, (6.16) can be expanded in the geometric series in one way or the other, and the result represents the count of states with $0=\left\{\mathbf{Q}_{+}, \overline{\mathbf{Q}}_{+}\right\}=$ $H_{m}-\mathbf{G}_{m}^{F}(m)$ for each pair of flavor and R-charges. It is interesting to note that the index simplifies when the $\boldsymbol{J}_{-}$-twist is turned off,

$$
\left.I\right|_{\mathbf{y}=1}=-1 .
$$

How can this happen?

In fact, this holds in general and is another consequence of $\mathcal{N}=4$ supersymmetry, which has another set of supercharges, $\mathbf{Q}_{-}$and $\overline{\mathbf{Q}}_{-}$with charge -1 and +1 under $\boldsymbol{J}_{-}$. They obey the lower part of the algebra (2.51), which yields the following identity on states obeying $0=H_{m}-\mathbf{G}_{m}^{F}(m)$ :

$$
\left\{\mathbf{Q}_{-}, \overline{\mathbf{Q}}_{-}\right\}=H_{m}+\mathbf{G}_{m}^{F}(m) \stackrel{\downarrow}{=} 2 \mathbf{G}_{m}^{F}(m) .
$$

Therefore, when $m \neq 0$, as far as the states that contribute to the index are concerned, $\mathbf{Q}_{-}+\overline{\mathbf{Q}}_{-}$defines a one to one correspondence between bosonic and fermionic states of non-zero flavor charge, while it vanishes on states of zero flavor charge. Therefore, $\left.I\right|_{\mathbf{y}=1}$ receives contribution only from the states of zero flavor charge, and hence is independent of the flavor twist parameter.

Let us illustrate these in a system with gauge symmetry. We take the non-compact version of Example 2: $\mathrm{U}(1)$ gauge theory with charge 1 chiral multiplets $X_{1}, \ldots, X_{N}$ and a charge $-d$ chiral multiplet $P$, and vanishing superpotential $W=0$. This system has $\mathrm{U}(N)$ flavor symmetry that acts in the standard way on $X_{1}, \ldots, X_{N}$. Positive $\zeta$ is the geometric 
phase with the total space of $\mathcal{O}(-d)$ over $\mathbf{C P}^{N-1}$ as the target. Negative $\zeta$ is the orbifold phase where we have $\mathbf{C}^{N} / \mathbf{Z}_{d}$. A generic element of the flavor group $g \in \mathrm{U}(N)$ has a compact set of fixed points - the zero section of $\mathcal{O}(-d)$ in the geometric phase and the origin of $\mathbf{C}^{N} / \mathbf{Z}_{d}$ in the orbifold phase. Special elements are those $g$ where $g^{d}$ has eigenvalue 1 , for which the fixed point set is non-compact - extending in the fibre direction of $\mathcal{O}(-d)$ or in the ray of $\mathbf{C}^{N} / \mathbf{Z}_{d}$. Let us compute the index with the twist parameter $y=\left(y_{1}, \ldots, y_{N}\right)$ for the maximal torus of $\mathrm{U}(N)$. It must be well-defined when $y$ is generic, i.e., when none of $y_{i}^{d}$ is equal to 1 . Assigning the trivial R-charge to all fields, we have

$$
\begin{aligned}
g & =\frac{1}{\mathbf{y}-\mathbf{y}^{-1}} \prod_{i=1}^{N}\left(\frac{x^{-\frac{1}{2}} y_{i}^{-\frac{1}{2}} \mathbf{y}-x^{\frac{1}{2}} y_{i}^{\frac{1}{2}} \mathbf{y}^{-1}}{x^{\frac{1}{2}} y_{i}^{\frac{1}{2}}-x^{-\frac{1}{2}} y_{i}^{-\frac{1}{2}}}\right) \cdot \frac{x^{\frac{d}{2}} \mathbf{y}-x^{-\frac{1}{2}} \mathbf{y}^{-1}}{x^{-\frac{d}{2}}-x^{\frac{d}{2}}} \\
& =(-1)^{N-1} \frac{\mathbf{y}^{1-N}}{\mathbf{y}-\mathbf{y}^{-1}} \prod_{i=1}^{N}\left(\frac{x-\varepsilon_{i} \mathbf{y}^{2}}{x-\varepsilon_{i}}\right) \cdot \frac{x^{d}-\mathbf{y}^{-2}}{x^{d}-1}
\end{aligned}
$$

where we introduced $y_{i}^{-1}=\varepsilon_{i}$ to simplify the expression. The index in the geometric phase $\zeta>0$ is

$$
I=(-1)^{N} \mathbf{y}^{2-N} \sum_{i=1}^{N} \prod_{j \neq i}\left(\frac{\varepsilon_{i}-\varepsilon_{j} \mathbf{y}^{2}}{\varepsilon_{i}-\varepsilon_{j}}\right) \cdot \frac{\varepsilon_{i}^{d}-\mathbf{y}^{-2}}{\varepsilon_{i}^{d}-1} .
$$

The diagonals $\varepsilon_{i}=\varepsilon_{j}$ for $i \neq j$ are only apparent singularity. For example,

$$
I_{N=2}=\frac{\left(\varepsilon_{1} \varepsilon_{2} s_{d-2}\left(\varepsilon_{1}, \varepsilon_{2}\right)+1\right) \mathbf{y}^{-1}+\left(\varepsilon_{1}^{d} \varepsilon_{2}^{d}-1\right)+\left(\varepsilon_{1} \varepsilon_{2} s_{d-2}\left(\varepsilon_{1}, \varepsilon_{2}\right)+\varepsilon_{1}^{d} \varepsilon_{2}^{d}\right) \mathbf{y}}{\left(\varepsilon_{1}^{d}-1\right)\left(\varepsilon_{2}^{d}-1\right)}
$$

where $s_{d-2}\left(\varepsilon_{1}, \varepsilon_{2}\right)=\left(\varepsilon_{1}^{d-1}-\varepsilon_{2}^{d-1}\right) /\left(\varepsilon_{1}-\varepsilon_{2}\right)$. The index has singularity only at $\varepsilon_{i}^{d}=1$ for some $i$, as expected. The index in the orbifold phase $\zeta<0$ can be obtained from this by the wall crossing formula (5.3):

$$
I_{\text {orbifold }}=I_{\text {geometric }}+(-1)^{N-1}\left(\mathbf{y}^{-(N-2)}+\cdots+\mathbf{y}^{N-2}\right) .
$$

Recall that the wall crossing part is independent of the flavor twist, even when the theory is non-compact, since the non-compactness of the Higgs branch does not affect the effective compactness of the Coulomb branch. When we turn off the $\boldsymbol{J}_{-}$twist, we have

$$
\left.I_{\text {geometric }}\right|_{\mathbf{y}=1}=(-1)^{N} N,\left.\quad I_{\text {orbifold }}\right|_{\mathbf{y}=1}=(-1)^{N} .
$$

Indeed they are independent of the flavor twist parameters. Up to sign, the result agrees with the Euler number of the fixed point set - the zero section $\left(\mathbf{C P}^{N-1}\right)$ in the geometric phase and the origin (one point) in the orbifold phase. Why is that? The real mass provides a quadratic potential in the direction transverse to the fixed point set $X^{F}$, and hence at energies below the mass, the theory is well approximated by the sigma model on $X^{F}$, whose index is $(-1)^{\operatorname{dim} X^{F}} \chi\left(X^{F}\right)$. The trivial action of $\mathbf{Z}_{d}$ on the origin in the orbifold phase might not cause a problem in $1 \mathrm{~d}$ where there is no twisted sectors. How about the sign? In the limit of very large masses, the theory of the transverse modes can 


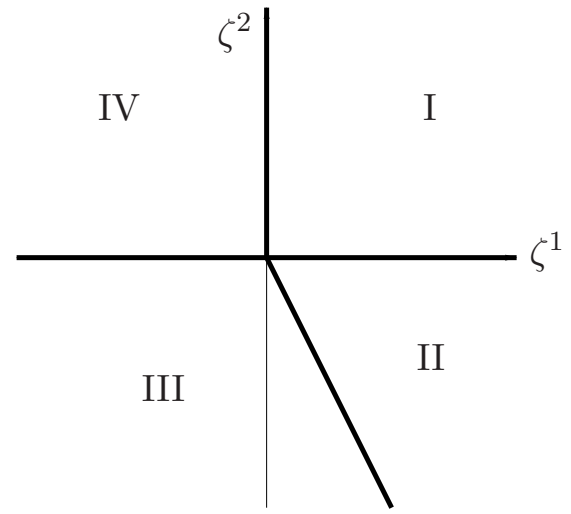

Figure 11. The phases of "the two parameter model".

be well approximated by the direct product of decoupled copies of a free chiral multiplet with a large real mass. Using the result (6.17) for the individual component, we obtain $(-1)^{\operatorname{codim} X^{F}}$ as the index. This explains the above result, $(-1)^{1} \cdot(-1)^{N-1} N$ for the geometric phase and $(-1)^{N} \cdot 1$ in the orbifold phase.

From this exercise, we can extract the following general statement. Suppose an $\mathcal{N}=4$ model has an effective theory on the Higgs branch, which is a non-linear sigma model on an orbifold $X$. Suppose there is a continuous flavor group whose generic element has a compact set of fixed points $X^{F}$. Then the index is well defined if it is twisted by the flavor symmetry, and as we have learned above, the flavor twist dependence drops out when the $\boldsymbol{J}_{-}$-twist is turned off. In particular, $\left.I\right|_{\mathbf{y}=1}=I\left(y^{\mathbf{G}^{F}}\right)$ must be an integer. Let us assume that the local orbifold group acts trivially on $X^{F}$. Then, we have

$$
I\left(y^{\mathbf{G}^{F}}\right)=\sum_{i}(-1)^{\operatorname{dim} X} \chi\left(X_{i}^{F}\right)
$$

where the sum is over the connected components of $X^{F}$. When $X$ is a compact manifold, the index is independent of the flavor twist and its value at $\mathbf{y}=1$ is $(-1)^{\operatorname{dim} X} \chi(X)$. Thus, (6.24) is nothing but the Hopf theorem. Therefore, (6.24) can be regarded as a generalization of the Hopf theorem to $\mathcal{N}=4$ linear sigma models with non-compact Higgs branches. From the derivation, the statement itself must hold for any $\mathcal{N}=4$ non-linear sigma model on an orbifold $X$, even without linear sigma model realization.

\section{5 "The two parameter model"}

The next example is the model with gauge group $\mathrm{U}(1) \times \mathrm{U}(1)$, the following matter content

\begin{tabular}{|l|cccc|c|}
\hline & $P$ & $X_{1,2}$ & $Y_{1,2,3}$ & $Z$ & $\mathrm{FI}$ \\
\hline $\mathrm{U}(1)_{1}$ & -4 & 0 & 1 & 1 & $\zeta^{1}$ \\
$\mathrm{U}(1)_{2}$ & 0 & 1 & 0 & -2 & $\zeta^{2}$ \\
\hline
\end{tabular}

and the superpotential $W=P \cdot f(X, Y, Z)$ where $f(X, Y, Z)$ is a polynomial of charge $(4,0)$. This is the $1 \mathrm{~d}$ version of the $2 \mathrm{~d}(2,2)$ model which is popular among physicists [57]. 
There are four phases as shown in figure 11. I is a geometric phase, II is an orbifold phase, III is a Landau-Ginzburg orbifold phase and IV is a hybrid phase. The geometry in Phase I is a smooth compact Calabi-Yau threefold embedded as a hypersurface is a toric fourfold of $h^{1,1}=2$.

We may set $R_{P}=2$ and $R_{X}=R_{Y}=R_{Z}=0$. Then the $g$ function is

$$
\begin{gathered}
g=\left(\frac{1}{2 i \sin (\pi \mathbf{z})}\right)^{2} \frac{\sin \left(-\pi\left(-4 u_{1}\right)\right)}{\sin \left(\pi\left(-4 u_{1}+\mathbf{z}\right)\right)}\left(\frac{\sin \left(-\pi\left(u_{2}-\mathbf{z}\right)\right)}{\sin \left(\pi u_{2}\right)}\right)^{2} \\
\left(\frac{\sin \left(-\pi\left(u_{1}-\mathbf{z}\right)\right)}{\sin \left(\pi u_{1}\right)}\right)^{3} \frac{\sin \left(-\pi\left(u_{1}-2 u_{2}-\mathbf{z}\right)\right)}{\sin \left(\pi\left(u_{1}-2 u_{2}\right)\right)}
\end{gathered}
$$

Below we write the result of residue computation in each phase.

- Phase I:

$$
I=84 \mathbf{y}^{-1}+84 \mathbf{y}
$$

- Phase II:

$$
I=82 \mathbf{y}^{-1}+82 \mathbf{y}
$$

- Phase III:

$$
I=\mathbf{y}^{-3}+83 \mathbf{y}^{-1}+83 \mathbf{y}+\mathbf{y}^{3} .
$$

- Phase IV:

$$
I=\mathbf{y}^{-3}+86 \mathbf{y}^{-1}+86 \mathbf{y}+\mathbf{y}^{3} .
$$

We see that non-trivial wall crossing is happening at each phase boundary. Since the gauge group is Abelian, all of them are simple walls. Let us present the mixed branch analysis of the wall crossing.

I $\rightarrow$ II. The unbroken gauge group at the mixed branch along this phase boundary is $G_{1}=\mathrm{U}(1)_{2}$ and the fields are decomposed as

$$
\begin{aligned}
& \text { (C) } X_{1,2}(1), Z(-2), \\
& \text { (H) } P(-4), Y_{1,2,3}(1) .
\end{aligned}
$$

As in section 3.4, to the right of each field, we put its charge under $G_{1}$ for (C) and $G / G_{1}$ for $(\mathrm{H})$. The Coulomb branch of $(\mathrm{C})$ has $N_{\mathrm{eff}}^{(4)}=2-1=1$ so that we have $\Delta_{C} I^{(\mathrm{C})}(\mathbf{y})=-1$. The Higgs branch of $(\mathrm{H})$ is the degree four hypersurface in $\mathbf{C P}^{2}$ : it is a curve of genus 3 and has $I^{(\mathrm{H})}(\mathbf{y})=2 \mathbf{y}^{-1}+2 \mathbf{y}^{1}$ (see section 6.2). Thus,

$$
\Delta I=\left(2 \mathbf{y}^{-1}+2 \mathbf{y}^{1}\right) \cdot(-1)=-2 \mathbf{y}^{-1}-2 \mathbf{y}^{1} .
$$

Note also that the wall crossing states, which go out since $N_{\mathrm{eff}}^{(4)}>0$, form the following Hodge diamond 
II $\rightarrow$ III. The unbroken gauge group is $G_{1}=\left\{\left(g^{2}, g\right)\right\}$ and the fields are decomposed as

(C) $P(-8), X_{1,2}(1), Y_{1,2,3}(2)$,

(H) $Z(1)$.

The Coulomb branch of $(\mathrm{C})$ has $N_{\text {eff }}^{(4)}=-1+2+3=4$ so that we have $\Delta_{C} I^{(\mathrm{C})}(\mathbf{y})=$ $\mathbf{y}^{-3}+\mathbf{y}^{-1}+\mathbf{y}^{1}+\mathbf{y}^{3}$. The Higgs branch of $(\mathrm{H})$ is one point and hence has $I^{(\mathrm{H})}(\mathbf{y})=1$. Thus,

$$
\Delta I=\mathbf{y}^{-3}+\mathbf{y}^{-1}+\mathbf{y}^{1}+\mathbf{y}^{3} .
$$

The wall crossing states, which go out since $N_{\text {eff }}^{(4)}>0$, form the Hodge diamond with $1,1,1,1$ in the vertical middle.

III $\rightarrow$ IV. The unbroken gauge group is $G_{1}=\mathrm{U}(1)_{2}$ and the fields are decomposed as

$$
\begin{aligned}
& \text { (C) } X_{1,2}(1), Z(-2), \\
& \text { (H) } P(-4), Y_{1,2,3}(1) .
\end{aligned}
$$

The Coulomb branch of $(\mathrm{C})$ has $N_{\mathrm{eff}}^{(4)}=-2+1=-1$ so that we have $\Delta_{C} I^{(\mathrm{C})}(\mathbf{y})=1$. The Higgs branch theory of $(\mathrm{H})$ is the Landau-Ginzburg orbifold $W=f_{4}(Y) / \mathbf{Z}_{4}$ where $f_{4}$ is a quartic polynomial. To simplify the analysis let us take the Fermat polynomial, for which the relations are $Y_{1}^{3}=Y_{2}^{3}=Y_{3}^{3}=0$. Since $\Omega=\mathrm{d} Y_{1} \wedge \mathrm{d} Y_{2} \wedge \mathrm{d} Y_{3}$ has charge 3, for $O(Y) \Omega$ to be $\mathbf{Z}_{4}$ invariant, $O(Y)$ must have charge $1(\bmod 4)$ under $\mathbf{Z}_{4}$. There are six states:

$$
Y_{1} \Omega, Y_{2} \Omega, Y_{3} \Omega, \quad Y_{1} Y_{2}^{2} Y_{3}^{2} \Omega, Y_{1}^{2} Y_{2} Y_{3}^{2} \Omega, Y_{1}^{2} Y_{2}^{2} Y_{3} \Omega
$$

The R-charge assignment which is conjugate symmetric is $-\frac{1}{2}$ for the first three and $\frac{1}{2}$ for the last three. Thus, $I^{(\mathrm{H})}(\mathbf{y})=3 \mathbf{y}^{-1}+3 \mathbf{y}^{1}$. Therefore, the change of the index is

$$
\Delta I=\left(3 \mathbf{y}^{-1}+3 \mathbf{y}^{1}\right) \cdot 1=3 \mathbf{y}^{-1}+3 \mathbf{y}^{1} .
$$

The wall crossing states, which come in since $N_{\mathrm{eff}}^{(4)}<0$, form the Hodge diamond with $0,3,3,0$ in the horizontal middle.

IV $\rightarrow$ I. The unbroken gauge group is $G_{1}=\mathrm{U}(1)_{1}$ and the fields are decomposed as

(C) $P(-4), Y_{1,2,3}(1), Z(1)$,

(H) $X_{1,2}(1)$.

The Coulomb branch of $(\mathrm{C})$ has $N_{\mathrm{eff}}^{(4)}=1-3-1=-3$ so that we have $\Delta_{C} I^{(\mathrm{C})}(\mathbf{y})=$ $\mathbf{y}^{-1}+1+\mathbf{y}$. The Higgs branch of $(\mathrm{H})$ is $\mathbf{C P}^{1}$ which has $I^{(\mathrm{H})}(\mathbf{y})=-\mathbf{y}^{-1}-\mathbf{y}^{1}$. Thus,

$$
\Delta I=\left(-\mathbf{y}^{-1}-\mathbf{y}^{1}\right) \cdot\left(\mathbf{y}^{-1}+1+\mathbf{y}\right)=-\mathbf{y}^{-3}-2 \mathbf{y}^{-1}-2 \mathbf{y}^{1}-\mathbf{y}^{3} .
$$

The wall crossing states, which come in since $N_{\mathrm{eff}}^{(4)}<0$, form the Hodge diamond with $1,2,2,1$ in the vertical middle.

We see that $I_{\text {before }}+\Delta I=I_{\text {after }}$ indeed holds for each wall crossing. We have also determined the Hodge diamond formed by the wall crossing states. Using this, we can determine the Hodge diamonds of all four phases starting from the geometric phase, where the Hodge numbers are known by the Lefschetz hyperplane theorem: 
- Phase I (input):

$$
\begin{aligned}
& 1 \\
& \begin{array}{ll}
0 & 0
\end{array} \\
& \begin{array}{lll}
0 & 2 & 0
\end{array} \\
& \begin{array}{llll}
1 & 86 & 86 & 1
\end{array} \\
& \begin{array}{lll}
0 & 2 & 0
\end{array} \\
& 00 \\
& 1
\end{aligned}
$$

$\longrightarrow$ Phase II:

$$
\begin{aligned}
& 1 \\
& 00 \\
& \begin{array}{lll}
0 & 1 & 0
\end{array} \\
& \begin{array}{llll}
1 & 83 & 83 & 1
\end{array} \\
& \begin{array}{lll}
0 & 1 & 0
\end{array} \\
& \text { 0 } 0 \\
& 1
\end{aligned}
$$

$\longrightarrow$ Phase III:

$$
\begin{aligned}
& 0 \\
& 0 \quad 0 \\
& \begin{array}{lll}
0 & 0 & 0
\end{array} \\
& \begin{array}{llll}
1 & 83 & 83 & 1
\end{array} \\
& \begin{array}{lll}
0 & 0 & 0
\end{array} \\
& 00 \\
& 0
\end{aligned}
$$

$\longrightarrow$ Phase IV:

$$
\begin{aligned}
& 0 \\
& 0 \quad 0 \\
& \begin{array}{lll}
0 & 0 & 0
\end{array} \\
& \begin{array}{llll}
1 & 86 & 86 & 1
\end{array} \\
& \begin{array}{lll}
0 & 0 & 0
\end{array} \\
& 0 \quad 0 \\
& 0
\end{aligned}
$$

$\longrightarrow$ back to Phase I.

The Hodge diamond (6.37) of Phase III (Landau-Ginzburg orbifold phase) can be derived independently. First of all, the $2 \mathrm{~d}$ Hodge diamond is the same as (6.35) at all 
four phases because the model is Calabi-Yau. In the Landau-Ginzburg orbifold phase, it is known that the 1,2,2,1 in the diagonal middle and 3 out of the 86 in each of the two horizontal middle entries are twisted sector states (see e.g. section 2.1.1 of [58] based on [55]) and should not be taken in $1 \mathrm{~d}$. The others are untwisted sector states and should be included, thus giving (6.37).

There is an alternative approach to extract information on the ground states - it is to look at the "full Coulomb branch" where we take $\mathbf{x}$ to be large generic and integrate out all the matter multiplets. This induces an effective potential

$$
U_{\text {eff }}=\frac{e_{\text {eff }}^{2}}{2}\left(\zeta_{\text {eff }}\right)^{2}
$$

with $\zeta_{\text {eff }}=\zeta-\sum_{i} Q_{i} / 2\left|Q_{i}(\mathbf{x})\right|$, plus accompanying vector potential and Yukawa terms. The effective Lagrangian is reliable in the region where $|\mathbf{x}|$ is large enough $|\mathbf{x}|^{3} \gg e^{2}$. Since the bottom of the potential is typically at $|\mathbf{x}| \sim 1 /|\zeta|$, we need $e^{2}|\zeta|^{3} \ll 1$ to find a valid ground state on the full Coulomb branch. That is, the FI parameter must be very close to the origin $\zeta=0$. It is expected, however, that states that are found there will not disappear if we scale up $\zeta$. In the present example, the vacuum equation $\zeta_{\text {eff }}=0$ reads

$$
\zeta^{1}=\frac{1}{\left|\mathbf{x}_{1}\right|}+\frac{1}{2\left|\mathbf{x}_{1}-2 \mathbf{x}_{2}\right|}, \quad \zeta^{2}=\frac{1}{\left|\mathbf{x}_{2}\right|}-\frac{1}{\left|\mathbf{x}_{1}-2 \mathbf{x}_{2}\right|} .
$$

We see that there is no solution in Phases III and IV, while in each of Phases I and II, the solution space is well inside the safe region provided $|\zeta|$ is small enough. Applying the well-established routine of equivariant index counting, we find that the contribution of the full Coulomb branch vacua to the index is

$$
I_{\text {Coulomb }}=\left\{\begin{array}{lr}
-\mathbf{y}^{-3}-2 \mathbf{y}^{-1}-2 \mathbf{y}^{1}-\mathbf{y}^{3} & \text { Phase I } \\
-\mathbf{y}^{-3}-\mathbf{y}^{-1}-\mathbf{y}^{1}-\mathbf{y}^{3} & \text { Phase II } \\
0 & \text { Phases III and IV }
\end{array}\right.
$$

The Coulomb branch vacua form an $\mathrm{SU}(2)$ multiplet and are expected to have zero $\mathrm{U}(1)$ R-charge. We see that the result (6.41) correctly reproduces the vertical middle entries of the Hodge diamond at each phase.

It should be emphasized that the mixed branch analysis in section 3.4 and the above full Coulomb branch analysis are valid in totally different regimes and are good for different purposes. The former is valid deep inside the phase boundaries and can capture the full detail of the wall crossing states there, while the latter is valid only near the origin $\zeta=0$ and can capture a part of the SU(2) multiplets in each phase. For example, if we go far out in the I-IV phase boundary, i.e. $\zeta^{1} \rightarrow 0$ and $\zeta^{2} \rightarrow+\infty$, the vacuum equation (6.40) forces $\left|\mathbf{x}_{2}\right|$ to vanish, where the full Coulomb branch is totally invalid.

This is in sharp contrast to two-dimensional theories where it is necessary and valid to take into account the full Coulomb branch and all possible mixed branches at the same time, for a common purpose. For example, in the two-dimensional version of the present model [57], the vacuum equation on the full Coulomb branch reads

$$
\mathrm{e}^{t^{1}}=4^{4} \frac{\sigma_{1}}{\sigma_{1}-2 \sigma_{2}}, \quad \mathrm{e}^{t^{2}}=\frac{\left(\sigma_{1}-2 \sigma_{2}\right)^{2}}{\sigma_{2}^{2}},
$$




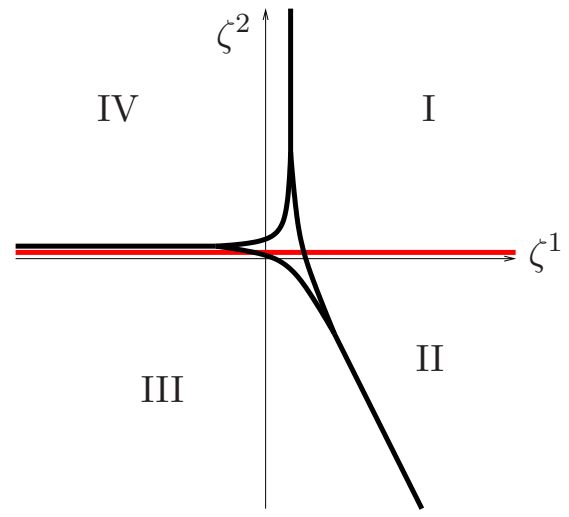

Figure 12. The $\zeta$-images of the discriminants (6.42) (black) and (6.43) (red).

where $t^{a}=\zeta^{a}-i \theta^{a}$ are the FI-theta parameter. Also, there is just one additional flat direction - from the mixed branch where $\mathrm{U}(1)_{2}$ is unbroken and $\mathrm{U}(1)_{1}$ is Higgsed which exists when

$$
\mathrm{e}^{t^{2}}=2^{2}
$$

(6.42) and (6.43) together define the discriminant locus in the FI-theta parameter space. Their $\zeta$-projection asymptote to the phase boundaries. See figure 12 . Note that the full Coulomb equation (6.42) allows valid solutions even in such asymptotic region. For example, the asymptotic region of I-IV phase boundary is $\mathrm{e}^{t^{1}} \rightarrow 4^{4}$ and $\mathrm{e}^{t^{2}} \rightarrow \infty$ : the vacuum equation does not force $\sigma_{2} \rightarrow 0$. We can take $\sigma_{1} \rightarrow \infty$ holding $\sigma_{2}$ fixed and large.

In one dimension, to account for the wall crossing deep inside phase boundaries, we only need to consider the corresponding mixed branches. This is supported by our index result which proves the wall crossing formula obtained just from the mixed branch analysis. There is no room for the full Coulomb branch to play any rôle there. However, the full Coulomb branch is the key element of the recent approach to index counting and wall crossing, in $\mathcal{N}=4$ quiver theories. We turn to that subject next.

\section{$7 \mathcal{N}=4$ quivers and quiver invariants}

Now we come to $\mathcal{N}=4$ quiver theories, which are very important as they address $4 \mathrm{~d}$ $\mathcal{N}=2$ wall-crossing phenomena. A given quiver theory is a low energy dynamics of particular charge sector, and our index computes the relevant protected spin character [59]

$$
\operatorname{Tr}\left[(-1)^{2 J_{3}} \mathbf{y}^{2\left(J_{3}+I_{3}\right)}\right]
$$

with $I_{3}$ and $J_{3}$ being the helicity operators of $\mathrm{SU}(2)_{R}$ and $\mathrm{SU}(2)$ little group, respectively. The trace is supposed to be taken after removing the universal half-hypermultiplet factor in the supermultiplet, so that the value is +1 for a half-hypermultiplet and $-\mathbf{y}-\mathbf{y}^{-1}$ for a vector multiplet. In particular the little group $\mathrm{SU}(2)$ of $d=4$ descends to $\mathrm{SU}(2)_{J}$ $R$-symmetry while $\mathrm{U}(1)_{I}$ is inherited from $d=4 \mathrm{SU}(2)_{R} R$-symmetry [30]. 
There has been extensive works for this class during last four years. For quivers without loops, the index computation in the Coulomb viewpoint have been carried out extensively, and lead to an explicit and recursive and partition-based index formulae $[26,29]$ based on a symplectic volume hypothesis. This was later re-derived in an ab initio computation from Seiberg-Witten theory [28]. For loopless quivers, it has been demonstrated [34] that the Coulomb branch analysis is sufficient in that the resulting index agrees with an independent Higgs computation and also with predictions coming from the Kontsevich-Soibelman algebra [35].

Quiver involving loops, and thus superpotentials, pose more challenges, as the Coulomb description often fails to capture all supersymmetric ground states. The additional states can be found from the Higgs side [23, 30, 38]. Some of these latter states, in particular, are impervious to wall-crossing and have been dubbed quiver invariants [30, 31]. These new kind of states serve as important building blocks in general BPS state construction. They are also believed to represent microstates of single-centered BPS black holes of $d=4$ $\mathcal{N}=2$ theories, generalizing and fine-tuning Ashoke Sen's earlier proposal to separate singe-center black holes from multi-center ones via angular momentum content [62].

With the new technology developed in this paper, we are now at a position to address most general quivers such as multi-loop quivers and non-Abelian quivers. In this section, we address two particular classes; all cyclic Abelian quiver where we reproduces results of ref. [31] and non-Abelian triangle quivers where we will also test the generalized Coulomblike expansion of refs. [60, 61], which fused the quiver invariants and the multi-center picture of BPS states into a single practical machinery.

\subsection{Abelian $(k+1)$-gon}

Let us start exploring Abelian $(k+1)$-gon with arrows $a=\left(a_{1}, a_{2}, \cdots, a_{k+1}\right)$, which will serve as a prototype. This class of theories were explored from both Higgs and Coulomb branches [30,38] and the complete equivariant indices computed [31, 32]. This also lead to the notion of quiver invariants that we will come back to after discussion of non-Abelian generalizations.

The model has gauge group $\mathrm{U}(1)^{k+1} / \mathrm{U}(1)_{\text {diag }}$, bifundamental matters $X_{1, \ldots, a_{i}}^{(i)}$ on the $i$-th edge and a generic linear combination of monomials of the form $X_{j_{k+1}}^{(k+1)} \cdots X_{j_{1}}^{(1)}$ as the superpotential. The gauge group is isomorphic to $\mathrm{U}(1)^{k}$, with $i \mathbf{t}^{*} \cong \mathbf{R}^{k}$, under which the $i$-th group of matters have charge $Q_{i}=e_{i}$ for $i=1, \ldots, k$, and the last group have charge $Q_{k+1}=-e_{1}-\cdots-e_{k}$. There are $(k+1)$ phases $\mathcal{C}_{1}, \ldots, \mathcal{C}_{k+1}$ where $\mathcal{C}_{i}$ is the positive span of all the charges except $Q_{i}$. We may assign R-charge 0 to the first $k$ groups of matters and 2 to the last group. Then, the $g$ function reads

$$
\begin{aligned}
& g=\left(\frac{1}{2 i \sin (\pi \mathbf{z})}\right)^{k} \times \\
& \times\left(\frac{\sin \left(-\pi\left(u_{1}-\mathbf{z}\right)\right)}{\sin \left(\pi u_{1}\right)}\right)^{a_{1}}\left(\frac{\sin \left(-\pi\left(u_{2}-\mathbf{z}\right)\right)}{\sin \left(\pi u_{2}\right)}\right)^{a_{2}} \cdots\left(\frac{\sin \left(-\pi\left(-u_{1}-\cdots-u_{k}\right)\right)}{\sin \left(\pi\left(-u_{1}-\cdots-u_{k}+\mathbf{z}\right)\right)}\right)^{a_{k+1}}
\end{aligned}
$$


In the phase $\mathcal{C}_{i}$, we must take the residue at the intersection of the singular hyperplanes of the fields except the $i$-group. For $\mathcal{C}_{k+1}$, it is the standard residue at the origin,

$$
I_{\mathcal{C}_{k+1}}=\int_{\left|u_{1}\right|=\cdots=\left|u_{k}\right|=\varepsilon} \mathrm{d}^{k} u g(u, \mathbf{z}) .
$$

For $\mathcal{C}_{i}$ with $i=1, \ldots, k$, it is the residue at $u_{j}=0(j \neq i)$ and $-u_{1}-\cdots-u_{k}+\mathbf{z}=0$. Because of the sign in front of $u_{i}$ of the last equation, we need to include an extra sign,

$$
I_{\mathcal{C}_{i}}=(-1) \int_{\substack{\left|u_{1}\right|=\cdots \hat{i} \cdots=\left|u_{k}\right|=\varepsilon \\\left|-u_{1}-\cdots-u_{k}+\mathbf{z}\right|=\varepsilon}} \mathrm{d}^{k} u g(u, \mathbf{z}) .
$$

This exactly reproduces the results of ref. [31] which were based on conventional index theorem for the Higgs branch, also demonstrating that the Higgs branch captures the entire index for this class of theories in all chambers.

As a simple example, consider the case $k=2$ and $a=(6,5,4)$. The is Example 3 in section 2.4 with rank vector $(1,1,1)$ and the number vector $(a, b, c)=(6,5,4)$. The three phases $\mathcal{C}_{1}, \mathcal{C}_{2}, \mathcal{C}_{3}$ are III, II, I respectively, in the notation of section 2.4 (see figure 3 ). The index in these phases are

$$
\begin{gathered}
I_{\mathrm{I}}=\int_{\left|u_{1}\right|=\left|u_{2}\right|=\varepsilon} \mathrm{d}^{2} u g(u, \mathbf{z})=-\frac{1}{\mathbf{y}^{5}}-\frac{2}{\mathbf{y}^{3}}+\frac{23}{\mathbf{y}}+23 \mathbf{y}-2 \mathbf{y}^{3}-\mathbf{y}^{5}, \\
I_{\mathrm{II}}=-\int_{\left|u_{1}\right|=\left|-u_{1}-u_{2}+\mathbf{z}\right|=\varepsilon} \mathrm{d}^{2} u g(u, \mathbf{z})=-\frac{1}{\mathbf{y}^{3}}+\frac{24}{\mathbf{y}}+24 \mathbf{y}-\mathbf{y}^{3}, \\
I_{\mathrm{III}}=-\int_{\left|u_{2}\right|=\left|-u_{1}-u_{2}+\mathbf{z}\right|=\varepsilon} \mathrm{d}^{2} u g(u, \mathbf{z})=\frac{25}{\mathbf{y}}+25 \mathbf{y} .
\end{gathered}
$$

In section 3.4, we worked out the I $\rightarrow$ II wall crossing of this example, as an illustration of the mixed branch analysis. The formula obtained there, (3.59), reads

$$
\Delta_{\mathrm{I} \rightarrow \mathrm{II}} I=(-1)\left(\mathbf{y}^{-5}+\mathbf{y}^{-3}+\mathbf{y}^{-1}+\mathbf{y}+\mathbf{y}^{3}+\mathbf{y}^{5}\right) \cdot(-1) .
$$

We see that $I_{\mathrm{I}}+\Delta_{\mathrm{I} \rightarrow \mathrm{II}} I=I_{\mathrm{II}}$ indeed holds. Of course, we can also look at the Hodge diamonds of the phases and the wall crossing states, and find agreement.

\subsection{Non-Abelian triangle quivers of ranks $(k, 1,1)$}

Now we will generalize this to non-Abelian examples. Here we will consider triangle quivers (Example 3, section 2.4). When a rank vector is $(k, 1,1)$ and intersection numbers are $(a, b, c)$, there exist four phases at most. See figure 4. The gauge group $[\mathrm{U}(k) \times \mathrm{U}(1) \times$ $\mathrm{U}(1)] / \mathrm{U}(1)_{\text {diag }}$ is isomorphic to $\mathrm{U}(1) \times \mathrm{U}(k)$ by $\left[g, \omega^{-1}, 1\right] \longleftrightarrow(\omega, g)$. With respect to a basis $\left\{e_{0} ; e_{1}, \ldots, e_{k}\right\}$ of $i t^{*}$, where $e_{0}$ is for the $\mathrm{U}(1)$ factor and $e_{\alpha}-e_{\beta}$ are the roots of $\mathrm{U}(k)$, the matter charges and the FI parameter are written as

$$
Q_{X}=e_{0}, \quad Q_{Y^{\alpha}}=e_{\alpha}, \quad Q_{Z_{\alpha}}=-e_{0}-e_{\alpha}
$$




$$
\zeta=\zeta^{1} e_{0}+\frac{\zeta^{2}}{k}\left(e_{1}+\cdots+e_{k}\right)
$$

Here $\zeta^{1}$ and $\zeta^{2}$ are as in (2.84). We assign R-charge 2 to $Z$ 's and 0 to others. Then, the $g$ function reads

$$
\begin{aligned}
g= & \left(\frac{1}{2 i \sin (\pi \mathbf{z})}\right)^{k+1} \prod_{\alpha \neq \beta} \frac{\sin \left(-\pi\left(u_{\alpha}-u_{\beta}\right)\right)}{\sin \left(\pi\left(u_{\alpha}-u_{\beta}-\mathbf{z}\right)\right)} \times \\
& \times\left(\frac{\sin \left(-\pi\left(u_{0}-\mathbf{z}\right)\right)}{\sin \left(\pi u_{0}\right)}\right)^{a} \prod_{\alpha=1}^{k}\left(\frac{\sin \left(-\pi\left(u_{\alpha}-\mathbf{z}\right)\right)}{\sin \left(\pi u_{\alpha}\right)}\right)^{b} \prod_{\alpha=1}^{k}\left(\frac{\sin \left(-\pi\left(-u_{\alpha}-u_{0}\right)\right)}{\sin \left(\pi\left(-u_{\alpha}-u_{0}+\mathbf{z}\right)\right.}\right)^{c} .
\end{aligned}
$$

In chamber I, $\zeta$ is a positive span of $Q_{X}$ and $Q_{Y^{1}}, \ldots, Q_{Y^{k}}$, as is evident from (7.9) and (7.8). A non-zero contribution comes only from the intersection of the corresponding hyperplanes, $\left(u_{0}, u_{1}, \ldots, u_{k}\right)=(0, \ldots, 0)$,

$$
I_{\mathrm{I}}=\frac{1}{k !} \int_{\left|u_{0}\right|=\left|u_{1}\right|=\cdots=\left|u_{k}\right|=\varepsilon} \mathrm{d}^{k+1} u g(u, \mathbf{z}) .
$$

In chamber II, $\zeta=\left(\zeta^{1}-\zeta^{2}\right) Q_{X}+\frac{\zeta^{2}}{k}\left(Q_{Z_{1}}+\cdots+Q_{Z_{k}}\right)$ is a positive span of $Q_{X}$ and $Q_{Z_{\alpha}}$ 's. A non-zero contribution comes only from the intersection of the corresponding hyperplanes, $\left(u_{0}, u_{1}, \ldots, u_{k}\right)=(0, \mathbf{z}, \ldots, \mathbf{z})$

$$
I_{\mathrm{II}}=\frac{(-1)^{k}}{k !} \int_{\left|u_{0}\right|=\left|-u_{1}-u_{0}+\mathbf{z}\right|=\cdots=\left|-u_{k}-u_{0}+\mathbf{z}\right|=\varepsilon} \mathrm{d}^{k+1} u g(u, \mathbf{z}) .
$$

In chamber IV, $\zeta=\left(-\zeta^{1}\right)\left(Q_{Y^{\alpha}}+Q_{Z_{\alpha}}\right)+\frac{\zeta^{2}}{k}\left(Q_{Y^{1}}+\cdots+Q_{Y^{k}}\right)$ is a positive span of $Q_{Y^{1}}, \ldots, Q_{Y^{k}}$ and $Q_{Y^{\alpha}}+Q_{Z_{\alpha}}$ for any $\alpha$, while in chamber III, $\zeta=\left(\zeta^{2}-\zeta^{1}\right)\left(Q_{Y^{\alpha}}+\right.$ $\left.Q_{Z_{\alpha}}\right)+\frac{-\zeta^{2}}{k}\left(Q_{Z_{1}}+\cdots+Q_{Z_{k}}\right)$ is a positive span of $Q_{Z_{1}}, \ldots, Q_{Z_{k}}$ and $Q_{Y^{\alpha}}+Q_{Z_{\alpha}}$ for any $\alpha$. In these chambers, a non-zero contribution can come only from the point $p$ with $\left(u_{0}, u_{1}, \ldots, u_{k}\right)=(\mathbf{z}, 0, \ldots, 0)$, which is a degenerate intersection where $2 k$ hyperplanes $H_{Y^{1}}, \ldots, H_{Y^{k}}, H_{Z_{1}}, \ldots, H_{Z_{k}}$ meet. At this point, we employ the constructive "definition" of the JK-residue [45]. We first choose an element $\xi \in i t^{*}$ which is in the same chamber as $\zeta$ with respect to the set $Q(p)=\left\{Q_{Y^{1}}, \ldots, Q_{Y^{k}}, Q_{Z_{1}}, \ldots, Q_{Z_{k}}\right\}$ and is generic with respect to all the partial sums of $Q(p)$. Then, we set

$$
\underset{p}{\operatorname{JK}-\operatorname{Res}}(Q(p), \zeta)=\sum_{F \in \mathcal{F} \mathcal{L}^{+}(Q(p), \xi)} \nu(F) \underset{F}{\operatorname{Res}},
$$

where the sum is over a certain set of flags of $i t^{*}$ determined by $Q(p)$ and $\xi$. See [45] or its review given in [40]. Let us put

$$
\xi(\epsilon):=\zeta+\epsilon\left(k e_{1}+(k-1) e_{2}+\cdots+2 e_{k-1}+e_{k}\right) .
$$

For a small but non-zero $\epsilon$, this belongs to the same chamber as $\zeta$ and is generic with respect to all the partial sums of $Q(p)$. In chamber IV, let us choose $\xi=\xi(\epsilon)$ with a small positive $\epsilon$. Then, the sum (7.13) consists of a single term with the flag

$$
\{0\} \subset \mathbf{R} Q_{Y^{1}} \subset \mathbf{R} Q_{Y^{1}}+\mathbf{R} Q_{Y^{2}} \subset \cdots \subset \mathbf{R} Q_{Y^{1}}+\cdots+\mathbf{R} Q_{Y^{k}} \subset i \mathbf{t}^{*},
$$


which yields

$$
I_{\mathrm{IV}}=\frac{(-1)}{k !} \underset{u_{0}=\mathbf{z}}{\operatorname{res}} \cdot \underset{u_{k}=0}{\operatorname{res}} \cdots \underset{u_{1}=0}{\operatorname{res}} g(u, \mathbf{z}) .
$$

In chamber III, let us choose $\xi=\xi(\epsilon)$ with a small negative $\epsilon$. Then, the sum (7.13) consists of a single term with the flag

$$
\{0\} \subset \mathbf{R} Q_{Z_{1}} \subset \mathbf{R} Q_{Z_{1}}+\mathbf{R} Q_{Z_{2}} \subset \cdots \subset \mathbf{R} Q_{Z_{1}}+\cdots+\mathbf{R} Q_{Z_{k}} \subset i \mathrm{t}^{*},
$$

which yields

$$
I_{\text {III }}=\frac{(-1)^{k+1}}{k !} \underset{u_{0}=\mathbf{z}}{\operatorname{res}} \cdot \underset{u_{k}=\mathbf{z}-u_{0}}{\operatorname{res}} \cdots \underset{u_{1}=\mathbf{z}-u_{0}}{\operatorname{res}} g(u, \mathbf{z}) .
$$

Let us present the result of computation for low values of $k$ and $(a, b, c)$. First, $k=2$. With $(a, b, c)=(4,1,4)$, three chambers are empty and

$$
\begin{aligned}
I_{\mathrm{I}} & =0, \\
I_{\mathrm{II}} & =-\frac{1}{\mathbf{y}^{5}}-\frac{2}{\mathbf{y}^{3}}-\frac{3}{\mathbf{y}}-3 \mathbf{y}-2 \mathbf{y}^{3}-\mathbf{y}^{5}, \\
I_{\mathrm{III}} & =0 \\
I_{\mathrm{IV}} & =0,
\end{aligned}
$$

which is consistent with the results derived previously from purely geometrical method [33]. Let us look at the cases where the indices are non-zero at all the four branches. When the condition (2.90) is obeyed, say $(a, b, c)=(7,3,4)$, the fourth phase boundary between III and IV is actually moot, and no decay should be possible there. In accordance we find, the indices at each chambers are

$$
\begin{aligned}
I_{\mathrm{I}} & =10 \\
I_{\mathrm{II}} & =\frac{1}{\mathbf{y}^{4}}+\frac{2}{\mathbf{y}^{2}}+13+2 \mathbf{y}^{2}+\mathbf{y}^{4}, \\
I_{\mathrm{III}} & =\frac{1}{\mathbf{y}^{2}}+11+\mathbf{y}^{2}, \\
I_{\mathrm{IV}} & =\frac{1}{\mathbf{y}^{2}}+11+\mathbf{y}^{2} .
\end{aligned}
$$

If we take $(a, b, c)=(7,4,5)$, for which $(2.90)$ is violated, the indices all four chambers are indeed different from one another as

$$
\begin{aligned}
I_{\mathrm{I}} & =50, \\
I_{\mathrm{II}} & =\frac{1}{\mathbf{y}^{4}}+\frac{2}{\mathbf{y}^{2}}+87+2 \mathbf{y}^{2}+\mathbf{y}^{4}, \\
I_{\mathrm{III}} & =\frac{1}{\mathbf{y}^{6}}+\frac{2}{\mathbf{y}^{4}}+\frac{4}{\mathbf{y}^{2}}+89+4 \mathbf{y}^{2}+2 \mathbf{y}^{4}+\mathbf{y}^{6}, \\
I_{\mathrm{IV}} & =\frac{1}{\mathbf{y}^{6}}+\frac{2}{\mathbf{y}^{4}}+\frac{4}{\mathbf{y}^{2}}+54+4 \mathbf{y}^{2}+2 \mathbf{y}^{4}+\mathbf{y}^{6} .
\end{aligned}
$$

Next, let us take $k=3$. For $(a, b, c)=(10,4,3)$, we find

$$
I_{\mathrm{I}}=-\frac{1}{\mathbf{y}^{3}}-\frac{2}{\mathbf{y}}-2 \mathbf{y}-\mathbf{y}^{3},
$$




$$
\begin{aligned}
I_{\mathrm{II}} & =0 \\
I_{\mathrm{III}} & =-\frac{1}{\mathbf{y}}-\mathbf{y}, \\
I_{\mathrm{IV}} & =-\frac{1}{\mathbf{y}}-\mathbf{y} .
\end{aligned}
$$

while for $k=3$ and $(a, b, c)=(10,5,3)$, we find

$$
\begin{aligned}
I_{\mathrm{I}} & =\frac{1}{\mathbf{y}^{6}}+\frac{2}{\mathbf{y}^{4}}-\frac{2}{\mathbf{y}^{2}}-7-2 \mathbf{y}^{2}+2 \mathbf{y}^{4}+\mathbf{y}^{6}, \\
I_{\mathrm{II}} & =0 \\
I_{\mathrm{III}} & =\frac{1}{\mathbf{y}^{4}}+\frac{1}{\mathbf{y}^{2}}+1+\mathbf{y}^{2}+\mathbf{y}^{4}, \\
I_{\mathrm{IV}} & =\frac{1}{\mathbf{y}^{4}}-\frac{4}{\mathbf{y}^{2}}-9-4 \mathbf{y}^{2}+\mathbf{y}^{4} .
\end{aligned}
$$

The final example is for $k=3$ and $(a, b, c)=(10,4,4)$, which does have its own quiver invariant. The indices are

$$
\begin{aligned}
I_{\mathrm{I}} & =20 \\
I_{\mathrm{II}} & =20 \\
I_{\mathrm{III}} & =\frac{1}{\mathbf{y}^{4}}+\frac{2}{\mathbf{y}^{2}}+22+2 \mathbf{y}^{2}+\mathbf{y}^{4}, \\
I_{\mathrm{IV}} & =\frac{1}{\mathbf{y}^{4}}+\frac{2}{\mathbf{y}^{2}}+22+2 \mathbf{y}^{2}+\mathbf{y}^{4} .
\end{aligned}
$$

Note that I-IV and II-III walls are simple for any $k$, and III-IV is also simple for $k=2$. There we can see how the simple wall crossing formula works.

$\mathbf{I} \rightarrow \mathbf{I V}$. The data of the mixed branch is

$$
\begin{aligned}
& \text { (C) } \mathrm{U}(1) \times\left\{\mathbf{1}_{k}\right\} X_{1, \ldots, a}(1), Z_{1, \ldots, k}^{1, \ldots, c}(-1) \text {, } \\
& \text { (H) } \quad \mathrm{U}(k) \quad Y_{1, \ldots, b}(\mathbf{k}) \text {. }
\end{aligned}
$$

(C) has $N_{\mathrm{eff}}^{(4)}=a-k c$ and (H) has Higgs branch $G(k, b)$. This yields

$$
\Delta I=I_{G(k, b)} \cdot(-1)^{a-k c} \operatorname{sign}(a-k c)\left(\mathbf{y}^{-|a-k c|+1}+\cdots+\mathbf{y}^{|a-k c|-1}\right) .
$$

II $\rightarrow$ III. The data of the mixed branch is

$$
\begin{aligned}
& \text { (C) }\left\{\left(\omega, \omega^{-1} \mathbf{1}_{k}\right)\right\} \\
& X_{1, \ldots, a}(1), Y_{1, \ldots, b}^{1, \ldots, k}(-1), \\
& \text { (H) } \mathrm{U}(k) \quad Z^{1, \ldots, c}(\overline{\mathbf{k}}) .
\end{aligned}
$$

(C) has $N_{\mathrm{eff}}^{(4)}=a-k b$ and (H) has Higgs branch $G(k, c)$. This yields

$$
\Delta I=I_{G(k, c)} \cdot(-1)^{a-k b} \operatorname{sign}(a-k b)\left(\mathbf{y}^{-|a-k b|+1}+\cdots+\mathbf{y}^{|a-k b|-1}\right) .
$$


IV $\rightarrow$ III $(k=\mathbf{2}$ case $)$. The data of the mixed branch is

(C) $\left\{\left(1,\left(\begin{array}{c}\omega_{1} \\ 1\end{array}\right)\right)\right\} \quad Y_{1, \ldots, b}^{1}(1), Z_{1}^{1, \ldots, c}(-1)$,

(H) $\mathrm{U}(1) \times \mathrm{U}(1) X_{1, \ldots, a}(0,-1), Y_{1, \ldots, b}^{2}(1,0), Z_{2}^{1, \ldots, c}(-1,1) ; W=\sum A_{h}^{i j} Z_{2}^{h} Y_{j}^{2} X_{i}$.

(C) has $N_{\mathrm{eff}}^{(4)}=b-c$ and $(\mathrm{H})$ has complete intersection $M_{b, c}^{a}(A)$ of $a$ hypersurfaces in $\mathbf{C P}^{b-1} \times \mathbf{C P}^{c-1}$ as its Higgs branch. Thus,

$$
\Delta I=I_{M_{b, c}^{a}(A)} \cdot(-1)^{b-c} \operatorname{sgn}(b-c)\left(\mathbf{y}^{-|b-c|+1}+\cdots+\mathbf{y}^{|b-c|-1}\right) .
$$

These wall crossing formulae work in all cases above. For example, for the I-IV wall in the model with $k=3,(10,5,3)$, we have $\Delta I=-I_{G(3,5)}=-\left(\mathbf{y}^{-6}+\mathbf{y}^{-4}+2 \mathbf{y}^{-2}+2+\right.$ $\left.2 \mathbf{y}^{2}+\mathbf{y}^{4}+\mathbf{y}^{6}\right)$, in agreement with (7.21). As another example, let us look at the IV-III wall in the model $k=2,(7,4,5)$, where $\Delta I=I_{M_{4,5}^{7}(A)}$. Note that $M_{4,5}^{7}(A)$ is the intersection of seven hyperplanes in a seven dimensional manifold $\mathbf{C P}^{3} \times \mathbf{C P}^{4}$ and hence is a set of points, as many as $\left(H_{1}+H_{2}\right)^{7}=35$. This matches with $(7.19)$, as $54+35=89$. The mixed branch analysis can also tell us on the Hodge diamond of the wall crossing states.

\subsection{Quiver invariants}

Now that we accumulated several examples, it is time to talk about quiver invariant. It is instructive to recall the explicit example given at the end of subsection 7.1. The index and in fact the full cohomology of this example can be worked out [31]. The Hodge diamond for the three chambers are

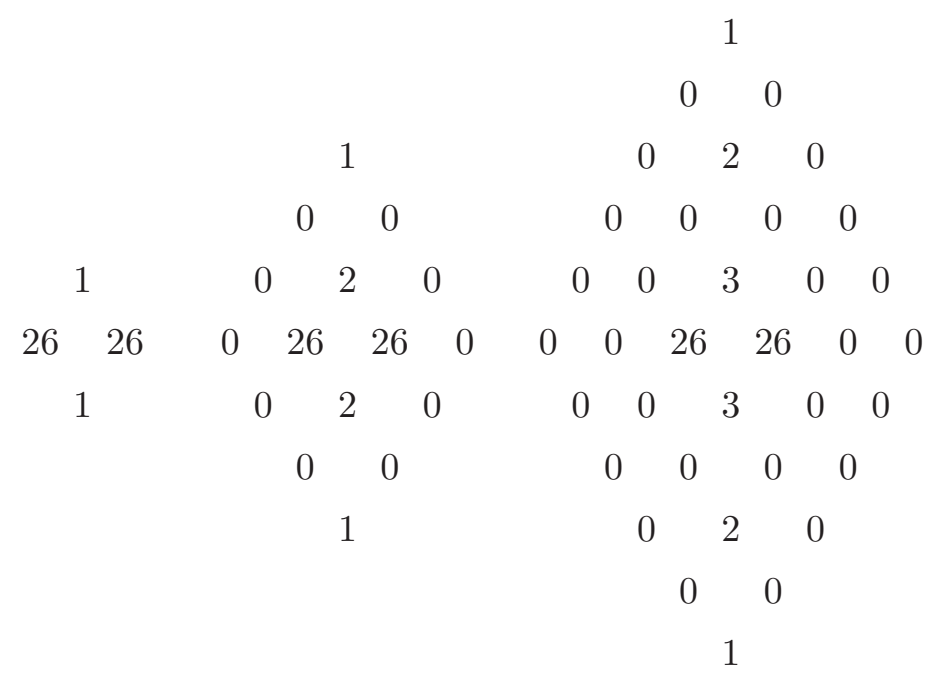

This set of Hodge diamond shows an interesting behavior. The dimensions of each chambers are different, and thus the size of the diamond. Yet, notice how the nontrivial entries along the horizontal middle remain the same throughout chambers. In fact, more detailed analysis of states shows that the entries along the vertical middle correspond to states that become destabilized at the walls, while these $26+26$ states remain stable even at the walls.

This kind of behavior has been observed for all cyclic Abelian quivers, as demonstrated precisely in refs. [30, 31]. For this class, the Hodge diamond consists of only two nontrivial 
lines, vertical middle and horizontal middle, just as in the above example; again, the vertical middle of the Hodge diamonds correspond to states that are detectable in the Coulomb description and are invariably become unstable at the walls, while states belonging to the horizontal middle are visible only to the Higgs description and impervious to wall-crossing instabilities. These wall-crossing-safe states have been dubbed "intrinsic Higgs states" [30] or "pure Higgs states" [38]. The part of index counting these states only shall be called quiver invariants, following refs. [30, 31], meaning that for any given they represents part of spectrum invariant across all chambers.

For these Abelian cyclic quiver, a state is either Coulomb-like, meaning that it becomes a multi-center bound state at a marginal stability wall, or "intrinsic Higgs." The split is clean, so we can define the quiver invariant as

$$
I=\Omega_{\mathrm{Inv}}+I_{\mathrm{Coulomb}} .
$$

In the above Abelian triangle with linking numbers $(6,5,4)$, for example, the invariant part of the index is

$$
\left.\Omega_{6,5,4}^{1,1,1}\right|_{\operatorname{Inv}}=\frac{26}{\mathbf{y}}+26 \mathbf{y}
$$

There is a potential ambiguity at the center of the Hodge diamond, i.e. $H^{(s / 2, s / 2)}$, when the vacuum manifold is of even complex dimension $s$. For these cyclic Abelian quivers, the ambiguity was neatly resolved by either the Lefshetz hyperplane theorem or by explicit counting/construction in the Coulomb description [30].

For general quiver theories, such neat separation of states into two classes cannot be true any more. As we saw earlier, for theories with rank large than 1, wall-crossing states are often of hybrid type, approximately a product of Coulomb-like multi-center wavefunction and tightly bound Higgs-like state. This implies that wall-crossing states, i.e. states that become unstable at a wall can involve quiver invariants of one or more subquivers as building blocks. Generally, given a quiver, one should find purely Coulomblike states which are multi-center states made from elementary centers, mixed states which are again multi-centered but with one or more centers belong to the quiver invariant of a subquiver, and then finally a single-centered wall-crossing-safe states, all contributing to the full index. The Hodge diamond will no longer be as simple as above, yet it is clear that there are states along the horizontal middle that are impervious to wall-crossing.

This idea, much in the spirit of mixed phase analysis we saw earlier, is physically appealing but complex to incorporate; ref. [60] proposed a routine to do this, although while keeping the quiver invariants as unknown input data. The idea is to break up a quiver to all possible subquivers, labeled $A$, and then consider the quiver invariant $\Omega_{\text {Inv }}^{A}$ for each of them. Each of these will act as a building block for wall-crossing states of the full quiver. Such states can no longer be considered invariant under wall-crossing, yet cannot be captured correctly if one only count the ordinary Coulombic states.

This tells us that the full index, if we view it from the wall-crossing perspective, will have to involve a sum over many partitions of the quiver into subquivers, say, $\left\{A_{i}\right\}$. 
Schematically, we have

$$
I=\sum_{\left\{A_{i}\right\}} I_{\left\{A_{i}\right\}}^{\text {Coulomb }} \times \prod_{i} \Omega_{\mathrm{Inv}}^{A_{i}}
$$

where each summand treats a subquiver $A_{i}$ as if it is a single charge center with the intrinsic degeneracy $\Omega_{\mathrm{Inv}}^{A_{i}}$, and the index $I_{\left\{A_{i}\right\}}^{\text {Coulomb }}$ counts the index of the resulting multi-center dynamics. Much of machinery developed in refs. [26, 28, 29] can be used not only for Coulomb branches of quiver theories but also for any multi-center bound state problems in $\mathbb{R}^{3}$ with four supersymmetries, so $I_{\left\{A_{i}\right\}}^{\text {Coulomb }}$ makes sense as a multi-center index. Computational procedure for $I^{\text {Coulomb }}$ that enter in this expansion is very involved but well-established. Because the index must correctly reflect the necessary Weyl projections, actual formulae are a bit more involved than this naive one; please see ref. [61] for the complete prescription.

The sum includes the case where the subquiver is the original quiver itself, which is precisely where wall-crossing-safe states of the original quiver enters and contributes a term $1 \times \Omega_{\mathrm{Inv}}$. In the other extreme, the sum also includes elementary subquivers, i.e., a single node with rank 1 , for each of which $\Omega_{\mathrm{Inv}}=1$. This shows that the quiver invariant generalizes the notion of intrinsic degeneracy of a hypermultiplet BPS particle to that of a single center BPS state of large internal degeneracy.

Theory by theory, isolating such invariant states is a well-defined, if tedious, exercise. All one needs to do is to solve for ground states and observe which states become unstable at marginal stability walls and which state never does. In particular, one can send all FI constant to "zero" where all marginal stability walls collide, leaving behind only invariant states. Systematic counting is a different matter since these theories are rather difficult to handle at vanishing FI parameters.

In view of this situation, one could choose a practical viewpoint and consider this equation as (recursive) definition of the quiver invariants. Then one begins to compute left hand sides, we can refer to the right hand side and extract quiver invariants. The invariant of one quiver will appear repeatedly in the index of larger quivers that can accommodate the former as a subquiver. As we compute the index of quivers with increasing complexity and ranks, we end up with a hugely over-determined problem therefore. The fact that we always get consistent answers for $\Omega_{\text {Inv }}$ in examples below nevertheless, goes a long way to confirm that the concept of the quiver invariant is robust and also that the above expansion scheme actually works and catalog faithfully all possible Coulombic, mixed, and invariant states.

Let us now scan through examples of the above triangle quivers. For rank $(2,1,1)$ and intersection numbers $(4,1,4)$, three of the four chambers are empty, implying vanishing quiver invariant. In fact, the same is true of rank $(1,1,1)$ version of the same quiver, and we find

$$
\left.\Omega_{4,1,4}^{2,1,1}\right|_{\text {Inv }}=0=\left.\Omega_{4,1,4}^{1,1,1}\right|_{\text {Inv }} .
$$

For intersections $(7,3,4)$, quiver invariant for rank $(1,1,1)$ is known to be null, and we have

$$
I_{\mathrm{I}, \mathrm{II}, \mathrm{III}, \mathrm{IV}}=I_{\mathrm{I}, \mathrm{II}, \mathrm{III}, \mathrm{IV}}^{\text {Coulomb }}+\left.\Omega_{7,3,4}^{2,1,1}\right|_{\mathrm{Inv}}
$$


from which we read off

$$
\left.\Omega_{7,3,4}^{2,1,1}\right|_{\text {Inv }}=9 .
$$

Next, with intersections $(7,4,5)$, both $(1,1,1)$ and $(2,1,1)$ quivers have nontrivial quiver invariants, and they show up for $(2,1,1)$ quiver index as

$$
I_{\mathrm{I}, \mathrm{IV}}=I_{\mathrm{I}, \mathrm{IV}}^{\text {Coulomb }}+\left.\Omega_{7,4,5}^{2,1,1}\right|_{\mathrm{Inv}}
$$

and

$$
I_{\mathrm{II}, \mathrm{III}}=I_{\mathrm{II}, \mathrm{III}}^{\text {Coulomb }}+1 \times\left.\Omega_{7,4,5}^{1,1,1}\right|_{\mathrm{Inv}}+\left.\Omega_{7,4,5}^{2,1,1}\right|_{\mathrm{Inv}} .
$$

Thus, we find

$$
\left.\Omega_{7,4,5}^{2,1,1}\right|_{\text {Inv }}=49,\left.\quad \Omega_{7,4,5}^{1,1,1}\right|_{\text {Inv }}=34,
$$

where the latter number is independently confirmed by studying rank $(1,1,1)$ quiver.

The least interesting examples among the rank $(3,1,1)$ quiver is the one with intersection numbers $(10,4,3)$, because for each ranks $(k \leq 3,1,1)$ there is at least one empty chambers. This is borne out in the index computation also, and we find

$$
\left.\Omega_{10,4,3}^{3,1,1}\right|_{\text {Inv }}=\left.\Omega_{10,4,3}^{2,1,1}\right|_{\text {Inv }}=\left.\Omega_{10,4,3}^{1,1,1}\right|_{\text {Inv }}=0
$$

and $I_{\mathrm{I}, \mathrm{II}, \mathrm{III}, \mathrm{IV}}$ are all purely Coulombic for $k=1,2,3$. Intersection numbers $(10,5,3)$ is a little more interesting. Although, rank $(3,1,1)$ and rank $(1,1,1)$ quivers both have an empty chamber so that

$$
\left.\Omega_{10,5,3}^{3,1,1}\right|_{\mathrm{Inv}}=0=\left.\Omega_{10,5,3}^{1,1,1}\right|_{\mathrm{Inv}} .
$$

The quiver invariant of the $k=2$ subquiver is nontrivial and enters the index of this $k=3$ quiver as

$$
I_{\mathrm{I}, \mathrm{IV}}=I_{\mathrm{I}, \mathrm{IV}}^{\text {Coulomb }}+\left(-\frac{1}{\mathbf{y}}-\mathbf{y}\right) \times\left.\Omega_{10,5,3}^{2,1,1}\right|_{\mathrm{Inv}}
$$

and

$$
I_{\mathrm{II}, \mathrm{III}}=I_{\mathrm{II}, \mathrm{III}}^{\text {Coulomb }} .
$$

Again comparison with the Coulomb computation shows,

$$
\left.\Omega_{10,5,3}^{2,1,1}\right|_{\text {Inv }}=\frac{6}{\mathbf{y}}+6 \mathbf{y}
$$

which is again independently verified by directly computing Coulomb and Higgs indices for the $(2,1,1)$ quiver with $(a, b, c)=(10,5,3)$. The final example is $(a, b, c)=(10,4,4)$, for which

$$
\left.\Omega_{10,4,4}^{3,1,1}\right|_{\text {Inv }}=19,\left.\quad \Omega_{10,4,4}^{2,1,1}\right|_{\text {Inv }}=\left.\Omega_{10,4,4}^{1,1,1}\right|_{\text {Inv }}=0 .
$$

All these examples exhibit the expected behavior of how the quiver invariants in a selfconsistent manner. 
The interesting dichotomy seen in the cyclic Abelian quivers, between the Coulomblike $\mathrm{SU}(2)_{R}$ multiplets and the invariant $\mathrm{U}(1)_{R}$ states suggest that we can compute not only the indices but even the full Hodge diamonds, through such recursive constructions of BPS states. ${ }^{9}$ Whether theses kind of ideas, including the notion of invariant part of spectrum and the recursive build-up of BPS states will work for more general gauged quantum mechanics remains to be seen.

\section{Systems with $\mathcal{N}=2$ supersymmetry}

In this section, we illustrate the index formula and wall crossing for $\mathcal{N}=2$ supersymmetric theories which do not have $\mathcal{N}=4$ supersymmetry. As explained in section 2.2, dependence on the flavor twist is the most interesting part.

\subsection{The $\mathrm{CP}^{N-1}$ model}

The first example is the $\mathcal{N}=2$ linear sigma model for $\mathbf{C} \mathbf{P}^{N-1}$ : the theory with gauge group $\mathrm{U}(1), N$ chiral multiplet and Wilson line of charge q, such that $\frac{N}{2}-\mathrm{q} \in \mathbf{Z}$. We have already computed the wall crossing formula $\Delta I$ in (5.9)-(5.11). Since there is no negatively charged chiral multiplets, we have $I=0$ for $\zeta<0$. Combining these, we find that $I=-\Delta I$ for $\zeta>0$, which agrees with the result in geometric phase (2.77)-(2.79).

Here we would like to compute the index (for $\zeta>0$ ) with the twist by the $\operatorname{SU}(N)$ flavor symmetry under which the chiral multiplets form the fundamental representation. The twist parameter is written as $\left(y_{1}, \ldots, y_{N}\right)$ obeying $y_{1} \cdots y_{N}=1$. Then, the $g$ function is

$$
g=\frac{x^{\mathrm{q}}}{\prod_{i=1}^{N}\left(x^{\frac{1}{2}} y_{i}^{\frac{1}{2}}-x^{-\frac{1}{2}} y_{i}^{-\frac{1}{2}}\right)}=\frac{x^{\mathrm{q}+\frac{N}{2}}}{\prod_{i=1}^{N}\left(x-\varepsilon_{i}\right)},
$$

where we write $y_{i}^{-1}=\varepsilon_{i}$ to simplify the expressions. Therefore, the index is

$$
\begin{aligned}
I & =\sum_{i=1}^{N} \frac{\varepsilon_{i}^{\mathrm{q}+\frac{N}{2}-1}}{\prod_{j \neq i}\left(\varepsilon_{i}-\varepsilon_{j}\right)} \\
& =\frac{\sum_{s \in \mathfrak{S}_{N}}(-1)^{\ell(s)} \varepsilon_{s(1)}^{\mathrm{q}+\frac{N}{2}-1} \varepsilon_{s(2)}^{N-2} \cdots \varepsilon_{s(N-1)}}{\prod_{i<j}\left(\varepsilon_{i}-\varepsilon_{j}\right)}
\end{aligned}
$$

For $\mathrm{q} \geq \frac{N}{2}$, the right hand side is the Weyl character formula ${ }^{10}$ for the irreducible

\footnotetext{
${ }^{9}$ We thank Ashoke Sen for bringing this aspect to our attention.

${ }^{10}$ The character of the irreducible representation of highest weight $\lambda$ of a simple Lie group is

$$
\chi_{\lambda}(\varepsilon)=\frac{\sum_{w \in W}(-1)^{\ell(w)} \varepsilon^{w(\lambda+\rho)}}{\varepsilon^{\rho} \prod_{\alpha>0}\left(1-\varepsilon^{-\alpha}\right)}
$$

where $\rho$ is half the sum of positive roots. For $\mathrm{SU}(N)$, we have $\varepsilon^{\rho}=\varepsilon_{1}^{N-1} \varepsilon_{2}^{N-2} \cdots \varepsilon_{N-1}$ and the denominator of this formula is $\prod_{i<j}\left(\varepsilon_{i}-\varepsilon_{j}\right)$.
} 
representation of $\mathrm{SU}(N)$ with highest weight $\varepsilon_{1}^{\mathrm{q}-\frac{N}{2}}$, i.e., the $\left(\mathrm{q}-\frac{N}{2}\right)$-th symmetric power of the fundamental representation $\mathbf{C}^{N}$. Since $\varepsilon_{i}=y_{i}^{-1}$, the actual representation is its dual, $S^{\mathrm{q}-\frac{N}{2}}\left(\mathbf{C}^{N}\right)^{*}$. In fact we had seen this already on the Coulomb branch (3.40). Also, this is consistent with the fact that holomorphic sections of $\mathcal{O}\left(q-\frac{N}{2}\right)$ over $\mathbf{C P}^{N-1}$ are polynomials of degree $\left(\mathrm{q}-\frac{N}{2}\right)$ of the homogeneous coordinates, and hence transform as $S^{\mathrm{q}-\frac{N}{2}}\left(\mathbf{C}^{N}\right)^{*}$ under $\mathrm{SU}(N)$. For $|\mathrm{q}|<\frac{N}{2}$, this vanishes since a pair of $\varepsilon_{i}$ 's have the same power in each term of the numerator on the right hand side, and hence there is a pairwise cancellation in the permutation sum. The vanishing $I=0$ can also be seen easily by deforming the $x$-integration contour to 0 and $\infty$. For $\mathrm{q} \leq-\frac{N}{2}$, it is better to write the first line of (8.2) in terms of $y_{i}$ 's as

$$
I=(-1)^{N-1} \sum_{i=1}^{N} \frac{y_{i}^{-\mathrm{q}+\frac{N}{2}-1}}{\prod_{j \neq i}\left(y_{i}-y_{j}\right)} .
$$

Up to the sign, this is the Weyl character of the representation $S^{-\mathrm{q}-\frac{N}{2}} \mathbf{C}^{N}$ of $\mathrm{SU}(N)$. This is consistent with the expressions of the wavefunctions on the Coulomb branch (3.41).

The above result is consistent with a particular case of the Borel-Weil-Bott theorem [65, $66]$ : as a representation of $\mathrm{SL}(N, \mathbf{C})$,

$$
H^{i}\left(\mathbf{C P}^{N-1}, \mathcal{O}(j)\right) \cong \begin{cases}S^{j}\left(\mathbf{C}^{N}\right)^{*} & i=0, j \geq 0 \\ S^{-j-N} \mathbf{C}^{N} & i=N-1, j \leq-N \\ 0 & \text { else. }\end{cases}
$$

\subsection{Grassmannian}

We next consider the model with gauge group $\mathrm{U}(k), N$ chiral multiplets in the fundamental representation, and the Wilson line in the $\operatorname{det}^{\mathrm{q}}$ representation. The anomaly free condition is $\mathrm{q}+\frac{N}{2} \in \mathbf{Z}$. When $N \geq k$, the chamber $\zeta>0$ is the geometric phase with the Grassmannian $G(k, N)$ supporting the line bundle $\mathfrak{F}=\mathcal{O}\left(\mathrm{q}-\frac{N}{2}\right)$. For $N<k$ or for $\zeta<0$, supersymmetry is broken.

Let us compute the index with the twist by the $\mathrm{SU}(N)$ flavor symmetry. The $g$ function is

$$
\begin{aligned}
g & =\prod_{a \neq b}\left(x_{a}^{\frac{1}{2}} x_{b}^{-\frac{1}{2}}-x_{a}^{-\frac{1}{2}} x_{b}^{\frac{1}{2}}\right) \frac{\left(x_{1} \cdots x_{k}\right)^{\mathrm{q}}}{\prod_{i=1}^{N} \prod_{a=1}^{k}\left(x_{a}^{\frac{1}{2}} y_{i}^{\frac{1}{2}}-x_{a}^{-\frac{1}{2}} y_{i}^{-\frac{1}{2}}\right)} \\
& =\prod_{a \neq b}\left(x_{a}-x_{b}\right) \frac{\left(x_{1} \cdots x_{k}\right)^{\mathrm{q}+\frac{N}{2}-k+1}}{\prod_{i, a}\left(x_{a}-\varepsilon_{i}\right)},
\end{aligned}
$$

where we set $y_{i}^{-1}=\varepsilon_{i}$. The singular hyperplanes are at $x_{a}=\varepsilon_{i}$ and the isolated intersections are where, for each $a, x_{a}=y_{i_{a}}$ for some $i_{a}$. Note that the residue vanishes when $i_{a}=i_{b}$ for $a \neq b$. In particular, the index vanishes when $N<k$ where this is 
unavoidable. Since we have $1 /|W|=1 /\left|\mathfrak{S}_{k}\right|$ in front, we just have to sum over a subset $\left\{i_{1}, \ldots, i_{k}\right\}$ of $\{1, \ldots, N\}$ consisting of $k$ distinct (unordered) elements:

$$
\begin{aligned}
I & =\sum_{\left\{i_{1}, \ldots, i_{k}\right\} \subset\{1, \ldots, N\}} \prod_{a \neq b}\left(\varepsilon_{i_{a}}-\varepsilon_{i_{b}}\right) \frac{\left(\varepsilon_{i_{1}} \cdots \varepsilon_{i_{k}}\right)^{\mathrm{q}+\frac{N}{2}-k}}{\prod_{a=1} \prod_{j \neq i_{a}}\left(\varepsilon_{i_{a}}-\varepsilon_{j}\right)} \\
& =\sum_{\substack{\mathrm{I} \subset\{1, \ldots, N\} \\
|\mathrm{I}|=k}} \frac{\left(\prod_{i \in \mathrm{I}} \varepsilon_{i}\right)^{\mathrm{q}+\frac{N}{2}-k}}{\prod_{i \in \mathrm{I}} \prod_{j \notin \mathrm{I}}\left(\varepsilon_{i}-\varepsilon_{j}\right)}=\frac{\sum_{s \in \mathfrak{S}_{N}}(-1)^{\ell(s)} s\left[\left(\varepsilon_{1} \cdots \varepsilon_{k}\right)^{\mathrm{q}-\frac{N}{2}} \varepsilon^{\rho}\right]}{\prod_{i<j}\left(\varepsilon_{i}-\varepsilon_{j}\right)} .
\end{aligned}
$$

For $\mathrm{q} \geq \frac{N}{2}$, the right hand side is the Weyl character formula for the irreducible representation of $\operatorname{SU}(N)$ with highest weight $\left(\varepsilon_{1} \cdots \varepsilon_{k}\right)^{\mathrm{q}-\frac{N}{2}}$. Since $\varepsilon_{i}=y_{i}^{-1}$, it is the character of the dual of that representation. By the Weyl dimension formula, the untwisted index is

$$
\left.I\right|_{y_{1}=\cdots y_{N}=1}=\prod_{i=1}^{k} \frac{\prod_{j=k+1}^{N}\left(\mathrm{q}-\frac{N}{2}+j-i\right)}{\prod_{j=k+1}^{N}(j-i)}=\prod_{i=1}^{k} \frac{\left(\mathrm{q}+\frac{N}{2}-i\right) !(k-i) !}{\left(\mathrm{q}-\frac{N}{2}-i\right) !(N-i) !} .
$$

For $|\mathrm{q}|<\frac{N}{2}$, the index vanishes, $I=0$, since a pair of $\varepsilon_{i}$ 's have the same power in each term of the numerator of (8.7), yielding pairwise cancellation of the permutation sum. For $\mathrm{q} \leq-\frac{N}{2}$, it is more convenient to use $y_{i}$ 's, in terms of which the middle expression of (8.7) can be written as

$$
I=(-1)^{k(N-k)} \sum_{\substack{\mathrm{I} \subset\{1, \ldots, N\} \\|\mathrm{I}|=k}} \frac{\left(\prod_{i \in \mathrm{I}} y_{i}\right)^{-\mathrm{q}+\frac{N}{2}-k}}{\prod_{i \in \mathrm{I}} \prod_{j \notin \mathrm{I}}\left(y_{i}-y_{j}\right)} .
$$

Up to the sign $(-1)^{k(N-k)}$, this is the character of the irreducible representation of $\operatorname{SU}(N)$ with the highest weight $\left(y_{1} \cdots y_{k}\right)^{-\mathrm{q}-\frac{N}{2}}$.

The above result is consistent with a particular case of the Borel-Weil-Bott theorem [65, $66]$ : as a representation of $\operatorname{SL}(N, \mathbf{C})$,

$$
H^{i}(G(k, N), \mathcal{O}(j)) \cong \begin{cases}V_{j\left(e_{1}+\cdots+e_{k}\right)}^{*} & i=0, j \geq 0 \\ V_{(-j-N)\left(e_{1}+\cdots+e_{k}\right)} & i=k(N-k), j \leq-N \\ 0 & \text { else. }\end{cases}
$$

\subsection{Distler-Kachru model}

We next consider the Distler-Kachru model (Example 2, section 2.4). We recall the data of the model in the table below:

\begin{tabular}{|c|crrc|}
\hline & $X_{1, \ldots, N}$ & $P$ & $\xi$ & $\eta^{1, \ldots, M}$ \\
\hline $\mathrm{U}(1)$ & 1 & $-\ell$ & $-d$ & $q_{1, \ldots, M}$ \\
$\mathrm{U}(1)_{F}$ & 0 & 1 & 0 & -1 \\
\hline
\end{tabular}


The $g$ function is

$$
g=\frac{\left(x^{\frac{d}{2}}-x^{-\frac{d}{2}}\right) \prod_{\alpha}\left(x^{-\frac{q_{\alpha}}{2}} y^{\frac{1}{2}}-x^{\frac{q_{\alpha}}{2}} y^{-\frac{1}{2}}\right)}{\left(x^{\frac{1}{2}}-x^{-\frac{1}{2}}\right)^{N}\left(x^{-\frac{\ell}{2}} y^{\frac{1}{2}}-x^{\frac{\ell}{2}} y^{-\frac{1}{2}}\right)}=(-1)^{M+1} y^{\frac{1-M}{2}} x^{-k} \frac{\left(x^{d}-1\right) \prod_{\alpha}\left(x^{q_{\alpha}}-y\right)}{(x-1)^{N}\left(x^{\ell}-1\right)}
$$

where

$$
k:=\frac{1}{2}\left(d+\sum_{\alpha} q_{\alpha}-N-\ell\right) .
$$

Note that the anomaly free condition is $k \in \mathbf{Z}$. Index computation is strightforward. Let us consider the case $M=N-1, q_{\alpha}=1, \ell=2$, in which case $k=\frac{d-3}{2}$ ( $d$ must be odd). Then, the index $I_{N, d}$ is, in the geometric phase,

$$
\begin{aligned}
& I_{2, d}^{+}=d, \\
& I_{3, d}^{+}=-d y^{-\frac{1}{2}}+d y^{\frac{1}{2}}, \\
& I_{4, d}^{+}=\frac{d^{3}+23 d}{24} y^{-1}-\frac{d^{3}-d}{12}+\frac{d^{3}-d}{24} y, \\
& I_{5, d}^{+}=-\frac{d^{3}+11 d}{12} y^{-\frac{3}{2}}+\frac{d^{3}-d}{6} y^{-\frac{1}{2}}-\frac{d^{3}-13 d}{12} y^{\frac{1}{2}}+0 \cdot y^{\frac{3}{2}},
\end{aligned}
$$

and in the Landau-Ginzburg phase,

$$
\begin{aligned}
I_{N, d}^{-} & =\frac{1}{2} y^{-\frac{d-1}{4}}\left(\frac{y^{\frac{d}{2}}-1}{y^{\frac{1}{2}}-1}+(-1)^{\frac{d-1}{2}+N} \frac{y^{\frac{d}{2}}+1}{y^{\frac{1}{2}}+1}\right) \\
& =\left\{\begin{array}{l}
y^{-\left[\frac{d-1}{4}\right]}+\cdots+y^{-1}+1+y+\cdots+y^{\left[\frac{d-1}{4}\right]} \quad N \text { even, } \\
y^{-\left[\frac{d+1}{4}\right]+\frac{1}{2}}+\cdots+y^{-\frac{1}{2}}+y^{\frac{1}{2}}+\cdots+y^{\left[\frac{d+1}{4}\right]-\frac{1}{2}} N \text { odd }
\end{array}\right.
\end{aligned}
$$

The simplicity in the Landau-Ginzburg phase is remarkable. Let us try to understand it. Recall that the model has gauge group $\mathbf{Z}_{\ell}=\mathbf{Z}_{2}$ and has chiral multiplets $X_{1}, \ldots, X_{N}$ of $\mathbf{Z}_{2} \times \mathrm{U}(1)_{F}$ charge $\left(1, \frac{1}{2}\right)$, a fermi multiplet $\xi$ of charge $\left(d,-\frac{d}{2}\right)$ and fermi multiplets $\eta^{1}, \ldots, \eta^{N-1}$ of charge $\left(1,-\frac{1}{2}\right)$. The $\mathrm{U}(1)_{F}$ charges are different from (8.11) but that is because $P$ has a non-zero value and its $\mathrm{U}(1)_{F}$ charge has to be cancelled by dressing it with the original $\mathrm{U}(1)$ gauge symmetry. The model also has the superpotential $\mathfrak{W}=f(X) \xi+\sum_{\alpha} g_{\alpha}(X) \eta^{\alpha}$. In the present model, $g_{\alpha}(X)$ are linear in $X$ and $f(X)$ is of degree $d$ in $X$. From the genericity requirement stated in section 2.4, we can find a coordinate such that $g_{1}(X)=X_{2}, \ldots, g_{N-1}(X)=X_{N}$, and that the coefficient of $X_{1}^{d}$ in $f(X)$ is non-zero. The space of supersymmetric ground states is the $\mathfrak{W}$-cohomology space before the orbifold projection, and it is easy to see that they are generated by elements of the form $X_{1}^{j}|0\rangle(j=0,1, \ldots, d-1)$, where $|0\rangle$ is the Clifford vacuum annihilated by $\xi$ and $\eta^{\alpha}$ 's. Orbifold projection is done by selecting $\mathbf{Z}_{2}$ invariant elements, but the problem is to find how $\mathbf{Z}_{2}$ acts on $|0\rangle$. To see this we look at the charge of the Clifford vacuum under the original $\mathrm{U}(1)$ gauge group. The vacuum $|0\rangle$ is annihilated also by $\psi_{i}$ 's and $\psi_{P}$. Then, the conjugate invariant charge assignment fixes the charge of $|0\rangle$ to be $N-\frac{d-1}{2}$. In this 
way, we find that the generator of $\mathbf{Z}_{2}$ acts as

$$
|0\rangle \longmapsto(-1)^{\frac{d-1}{2}+N}|0\rangle \text {. }
$$

This determines which of $X_{1}^{j}|0\rangle$ should be selected. It is evident that the resulting index, with $(-1)^{F}=1$ on $|0\rangle$, is equal to (8.18).

Let us see how the wall crossing happens, using our analysis on the Coulomb branch in section 3.3. We consider the case $N=2$ in the above series.

The index in the geometric phase is $d$ as in (8.14). This can be directly checked as follows. For $\zeta \gg 0$, the low energy theory is the non-linear sigma model on the hypersurface $f(X)=0$ of $\mathbf{C P}^{1}$ with the fermi multiplet with values in the kernel of $g_{1}(X)$. The hypersurface consists of $d$ distinct points for a generic choice of $f(X)$, and $g_{1}(X)$ is non-zero at each of them. Thus, the target space is the set of $d$ points and there is no fermi multiplet. We see that there are $d$ supersymmetric ground states which are all bosonic and have $\mathrm{U}(1)_{F}$ charge zero.

Let us now work out the Coulomb branch. The vacuum equation (3.47)-(3.48) reads

$$
\begin{array}{cl}
\sigma<0: & n_{X}=0, \quad \bar{n}_{P}=0, \quad m_{X}=0, \quad m_{P}=1, \\
& \left|\bar{n}_{X}\right|+2\left|n_{P}\right|+d m_{\xi}+\left(1-m_{\eta}\right)=k . \\
\sigma>0: & \bar{n}_{X}=0, \quad n_{P}=0, \quad\left|m_{X}\right|=2, \quad m_{P}=0, \\
& \left|n_{X}\right|+2\left|\bar{n}_{P}\right|+d\left(1-m_{\xi}\right)+m_{\eta}=k,
\end{array}
$$

Let us discuss each $d$ one by one.

When $d=1, k$ is negative, and there is no solution. That is, there should be no wall crossing. This is indeed consistent with $I_{2,1}^{+}=I_{2,1}^{-}=1$. Also, we have seen that there is a unique ground state of $\mathrm{U}(1)_{F}$ charge zero, for both $\zeta \gg 0$ and $\zeta \ll 0$. It is natural to expect that it is the unique ground state of the system all the way from $\zeta \gg 0$ to $\zeta \ll 0$.

When $d=3, k$ is zero, and the above equation has a unique solution with all $n$ 's zero. This means $N_{\text {eff }}=1>0$. Therefore, for $\zeta>0$, there is a unique Coulomb branch vacuum for each of $\sigma<0$ and $\sigma>0$, both bosonic and with $Q_{F}=0$. For $\zeta<0$ there is none. That is, there are two wall crossing states that go out as $\zeta$ goes from positive to negative. This is consistent with $I_{2,3}^{+}=3$ and $I_{2,3}^{-}=1$, and also to the fact that there are three supersymmetric ground states for $\zeta \gg 0$ and one for $\zeta \ll 0$, all with vanishing $\mathrm{U}(1)_{F}$ charge. As $\zeta$ goes from $+\infty$ to $-\infty$, two of the three ground states become wall crossing states and go out, while one of them stays till the end. We may write the process as

\begin{tabular}{|c|c|}
\hline$Q_{F}$ & 0 \\
\hline$\zeta \gg 0$ & $\circ \circ \circ$ \\
$\zeta \sim+0$ & $\circ ㅇ ㅡ$ \\
\hline$\zeta \sim-0$ & $\circ$ \\
$\zeta \ll$ & $\circ$ \\
\hline
\end{tabular}

Here, a white circle means a bosonic supersymmetric state and an underlined circle means a wall crossing state that goes out as $\zeta \searrow 0$. 
When $d=5$, then $k=1$, and there are six solutions in total, all having $N_{\text {eff }}>0$. Thus, they correspond to wall crossing states at small positive $\zeta$ that go out as $\zeta \searrow 0$. Their profiles are as follows:

\begin{tabular}{|c|cccc|}
\hline support & state & $(-1)^{F}$ & $Q_{F}$ number \\
\hline$\sigma<0$ & $|\sigma| \mathrm{e}^{\zeta \sigma} a_{\bar{X}}^{\dagger} \bar{\eta}_{P}|0\rangle_{\text {OSC }}$ & +1 & 0 & 2 \\
& $|\sigma|^{\frac{1}{2}} \mathrm{e}^{\zeta \sigma} \bar{\psi}_{P}|0\rangle_{\text {OSC }}$ & -1 & 1 & 1 \\
\multirow{5}{*}{$\sigma>0$} & $|\sigma| \mathrm{e}^{-\zeta \sigma} a_{X}^{\dagger} \bar{\xi} \bar{\psi}_{1} \bar{\psi}_{2} \bar{\lambda}|0\rangle_{\text {OSC }}$ & +1 & 0 & 2 \\
& $|\sigma|^{\frac{1}{2}} \mathrm{e}^{-\zeta \sigma} \bar{\xi} \bar{\eta} \bar{\psi}_{1} \bar{\psi}_{2} \bar{\lambda}|0\rangle_{\text {OSC }}$ & -1 & -1 & 1 \\
\hline
\end{tabular}

They induce the following change of the index,

$$
\Delta_{C} I\left(y^{Q_{F}}\right)=y^{-1}-4+y .
$$

This is consistent with $I_{2,5}^{+}=5$ and $I_{2,5}^{-}=y^{-1}+1+y$. However, the total number of states (six) is bigger than the number (five) of the ground states at the starting point, and some of them are even fermionic. This may be understood as follows: as $\zeta$ is decreased from large positive to small positive, two boson-fermion pairs of states, one pair with charge 1 and another pair with charge -1 , descend to have zero energy. As $\zeta \searrow 0$, four of the bosonic states of charge 0 and the fermionic states of charges 1 and -1 run away to infinity. For $\zeta<0$, there remain three bosonic states of charge $-1,0,1$ supported at the origin $\sigma=0$. This process may be written as

\begin{tabular}{|c|c|c|c|}
\hline$Q_{F}$ & -1 & 0 & 1 \\
\hline$\zeta \gg 0$ & & $\circ \circ \circ \circ \circ$ & \\
\hline$\zeta \sim+0$ & $\circ \bullet$ & $\circ \underline{\circ} \underline{\circ} \underline{\circ} \underline{\circ}$ & $\circ \bullet$ \\
\hline$\zeta \sim-0$ & ○ & $\circ$ & $\circ$ \\
\hline$\zeta \ll 0$ & $\circ$ & 0 & $\circ$ \\
\hline
\end{tabular}

Here, a black circle stands for a fermionic ground state.

We can continue in the same way. Here are the next few:

\begin{tabular}{|c|c|c|c|c|}
\hline & $Q_{F}$ & -1 & 0 & 1 \\
\hline$=7:$ & $\begin{array}{l}\zeta \gg 0 \\
\zeta \sim+0\end{array}$ & $\circ \underline{\circ} \bullet \underline{\bullet}$ & 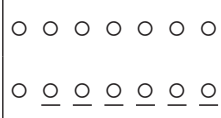 & $\circ \underline{\bullet} \underline{\bullet}$ \\
\hline & $\zeta \sim-0$ & ○ & $\circ$ & $\circ$ \\
\hline & $\zeta \ll 0$ & ○ & ○ & ○ \\
\hline
\end{tabular}

\begin{tabular}{|c|c|c|c|c|c|c|}
\hline & $Q_{F}$ & -2 & -1 & 0 & 1 & 2 \\
\hline$d=9:$ & $\begin{array}{l}\zeta \gg 0 \\
\zeta \sim+0\end{array}$ & & $\circ \underline{\circ} \underline{\circ} \bullet \bullet \bullet$ & $\begin{array}{c}9 \circ \text { ○'s } \\
\circ+8 \underline{\circ} \mathrm{s}\end{array}$ & $\circ \underline{\circ} \circ \bullet \bullet \bullet$ & \\
\hline & $\zeta \sim-0$ & $\bar{o}$ & o & $\circ$ & ० & $\bar{o}$ \\
\hline & $\zeta \ll 0$ & ० & ○ & 0 & 0 & ○ \\
\hline
\end{tabular}




$d=11:$\begin{tabular}{|c|c|c|c|c|c|}
\hline$Q_{F}$ & -2 & -1 & 0 & 1 & 2 \\
\hline$\zeta \gg 0$ & & & $11 \circ$ 's & & \\
$\zeta \sim+0$ & $\circ \circ \bullet \bullet$ & $\circ+3 \underline{\circ}, \mathrm{s}+4 \underline{\bullet}^{\prime} \mathrm{s}$ & $\circ+10 \stackrel{\circ}{\prime} \mathrm{s}$ & $\circ+3 \underline{\circ} \mathrm{\prime}, \mathrm{s}+4 \underline{\bullet} \mathrm{s}$ & $\circ \circ \bullet \bullet \bullet$ \\
\hline$\zeta \sim-0$ & $\circ \circ-\bullet$ & $\circ$ & $\circ$ & $\circ$ & $\circ \circ-\bullet$ \\
$\zeta \ll 0$ & $\circ$ & $\circ$ & $\circ$ & $\circ$ & $\circ$ \\
\hline
\end{tabular}

Overlined circles are wall crossing states that come in as $\zeta$ enters the negative region. (Equivalently, states that go out as $\zeta \nearrow 0$.) To be precise, there is an ambiguity in $d=7$ and $d=9$. The $d=7, Q_{F}= \pm 1$ and $d=9, Q_{F}= \pm 2$ entries involve $N_{\text {eff }}=0$ states, whose fate is not clear just from the Coulomb branch analysis. The above is a minimal possibility. The actual process could have been another minimal possibility:

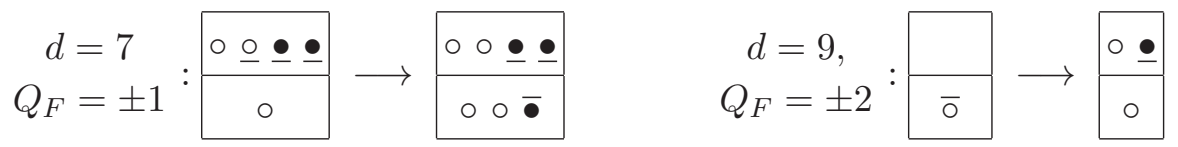

\subsection{Triangle quiver}

We consider the $\mathcal{N}=2$ triangle quiver, with the gauge group $G=\mathrm{U}(1)_{1} \times \mathrm{U}(1)_{2}$, three groups of chiral and three groups of fermi multiplets,

\begin{tabular}{|c|cccccc|}
\hline & $X_{1, \ldots, a}$ & $Y_{1, \ldots, b}$ & $Z_{1, \ldots, c}$ & $\eta_{3}^{1, \ldots, M_{3}}$ & $\eta_{1}^{1, \ldots, M_{1}}$ & $\eta_{2}^{1, \ldots, M_{2}}$ \\
\hline $\mathrm{U}(1)_{1}$ & 1 & 0 & -1 & -1 & 1 & 0 \\
$\mathrm{U}(1)_{2}$ & 0 & 1 & -1 & -1 & 0 & 1 \\
\hline $\mathrm{U}(1)_{F_{1}}$ & 1 & 0 & 0 & -1 & 0 & -1 \\
$\mathrm{U}(1)_{F_{2}}$ & 0 & 1 & 0 & -1 & -1 & 0 \\
\hline
\end{tabular}

and the superpotential

$$
\mathfrak{W}=\sum_{\gamma=1}^{M_{3}} f_{3 \gamma}(X, Y) \eta_{3}^{\gamma}+\sum_{\alpha=1}^{M_{1}} f_{1 \alpha}(Y, Z) \eta_{1}^{\alpha}+\sum_{\beta=1}^{M_{2}} f_{2 \beta}(Z, X) \eta_{2}^{\beta},
$$

where $f_{3 \gamma}(X, Y), f_{1 \alpha}(Y, Z)$ and $f_{2 \beta}(Z, X)$ are generic bilinear polynomials of the two entries. The system has $\mathrm{U}(1)_{F_{1}} \times \mathrm{U}(1)_{F_{2}}$ flavor symmetry whose charges had been listed above in advance. The anomaly free condition is

$$
a+c+M_{3}+M_{1} \in 2 \mathbf{Z}, \quad b+c+M_{3}+M_{2} \in 2 \mathbf{Z} .
$$

The D-term equations read

$$
\|X\|^{2}-\|Z\|^{2}=\zeta^{1}, \quad\|Y\|^{2}-\|Z\|^{2}=\zeta^{2},
$$

and the F-term equations read

$$
f_{3 \gamma}(X, Y)=0 \forall \gamma, \quad f_{1 \alpha}(Y, Z)=0 \forall \alpha, \quad f_{2 \beta}(Z, X)=0 \forall \beta .
$$


The theory has three phases, just as in figure 3, and their nature depends very much on the numbers $(a, b, c)$ and $\left(M_{3}, M_{1}, M_{2}\right)$. The $g$ function reads

$$
\begin{aligned}
& g=\frac{\left(2 i \sin \left(\pi\left(u_{1}+u_{2}+z_{1}+z_{2}\right)\right)\right)^{M_{3}}\left(2 i \sin \left(\pi\left(-u_{1}+z_{2}\right)\right)\right)^{M_{1}}\left(2 i \sin \left(\pi\left(-u_{2}+z_{1}\right)\right)\right)^{M_{2}}}{\left(2 i \sin \left(\pi\left(u_{1}+z_{1}\right)\right)\right)^{a}\left(2 i \sin \left(\pi\left(u_{2}+z_{2}\right)\right)\right)^{b}\left(2 i \sin \left(\pi\left(-u_{1}-u_{2}\right)\right)\right)^{c}} \\
&=(-1)^{c+M_{2}+M_{3}} y_{1}^{-\frac{a+M_{2}+M_{3}}{2}} y_{2}^{-\frac{b+M_{1}+M_{3}}{2}} \times \\
& x_{1}^{\frac{a+c-M_{1}-M_{3}}{2}} x_{2}^{\frac{b+c-M_{2}-M_{3}}{2}} \frac{\left(x_{1} x_{2} y_{1} y_{2}-1\right)^{M_{3}}\left(x_{1}-y_{2}\right)^{M_{1}}\left(x_{2}-y_{1}\right)^{M_{2}}}{\left(x_{1}-y_{1}^{-1}\right)^{a}\left(x_{2}-y_{2}^{-1}\right)^{b}\left(x_{1} x_{2}-1\right)^{c}}
\end{aligned}
$$

We consider the case $(a, b, c)=(1,3,3),\left(M_{3}, M_{1}, M_{2}\right)=(2,2,2)$ in detail. In Phase I, the vacuum equations require $Z=0$ and that $(X, Y)$ is at a point of $\mathbf{C P}^{2}$ determined by $f_{31}(X, Y)=f_{32}(X, Y)=0$. Of the six fermi multiplets, five mixes with the fermions of the chiral multiplets and only one of the $\eta_{1}^{1,2}$ remains massless. With respect to the two flavor symmetries, which are modified by the gauge symmetry so that $X$ and $Y$ are neutralized, $\eta_{1}^{1,2}$ has charge $(-1,-1)$. Therefore, the index is

$$
I_{\mathrm{I}}=y_{1}^{\frac{1}{2}} y_{2}^{\frac{1}{2}}-y_{1}^{-\frac{1}{2}} y_{2}^{-\frac{1}{2}} .
$$

Phase II has a similar structure, and the index is the same as in Phase I,

$$
I_{\mathrm{II}}=y_{1}^{\frac{1}{2}} y_{2}^{\frac{1}{2}}-y_{1}^{-\frac{1}{2}} y_{2}^{-\frac{1}{2}} .
$$

In Phase III, the vacuum equations require $X=0$ and that $(Y, Z)$ is in the complete intersection $\mathcal{X}$ of the hypersurfaces $f_{11}(Y, Z)=f_{12}(Y, Z)=0$ in $\mathbf{C P}^{2} \times \mathbf{C P}^{2} .(\mathcal{X}$ is the del Pezzo surface of degree 6 - the blow up at three general points of $\mathbf{C P}^{2}$.) The fermions $\eta_{1}^{1,2}$ mixes with the superpartner of the two transverse directions of $\mathcal{X}$ in $\mathbf{C P}^{2} \times \mathbf{C P}^{2}$. Of the four remaining fermions, $\eta_{3}^{1,2}$ and $\eta_{2}^{1,2}$, one mixes with the superpartner of $X$ and we are left with the three spanning the vector bundle $\mathcal{E}$ defined by

$$
0 \rightarrow \mathcal{E} \longrightarrow \mathcal{O}(0,1)^{\oplus 2} \oplus \mathcal{O}(1,0)^{\oplus 2} \stackrel{\left(f_{3}(Y), f_{2}(Z)\right)}{\longrightarrow} \mathcal{O}(1,1) \rightarrow 0 .
$$

Ground states correspond to Dolbeault cohomology classes with values in ${ }^{11}$

$$
\begin{aligned}
\mathfrak{F} & =\sqrt{K} \mathcal{X} \otimes \operatorname{det}^{-\frac{1}{2}} \mathcal{E} \otimes \wedge \mathcal{E}=(\wedge \mathcal{E})(-1,-1) \\
& =\mathcal{O}(-1,-1) \oplus \mathcal{E}(-1,-1) \oplus\left(\wedge^{2} \mathcal{E}\right)(-1,-1) \oplus \mathcal{O}
\end{aligned}
$$

Using Riemann-Roch formula, we find that the Dolbeault indices of the four terms of the last expression are $1,-1,-1,1$ respectively. Since $\eta_{3}$ 's and $\eta_{2}$ 's both have the $\mathrm{U}(1)_{F_{1}} \times \mathrm{U}(1)_{F_{2}}$ flavor charge $(-1,-1)$, again after neutralizing $y$ and $z$, we find that the index in this phase is

$$
I_{\mathrm{III}}=y_{1}^{\frac{3}{2}} y_{2}^{\frac{3}{2}}+y_{1}^{\frac{1}{2}} y_{2}^{\frac{1}{2}}-y_{1}^{-\frac{1}{2}} y_{2}^{-\frac{1}{2}}-y_{1}^{-\frac{3}{2}} y_{2}^{-\frac{3}{2}} .
$$

It is straightforward to check that the above indices in the three phases agrees with the ones obtained from (8.35) by the residue computation.

\footnotetext{
${ }^{11}$ In the final equality, we use $\wedge^{3} \mathcal{E}=\mathcal{O}(1,1)$ that follows from (8.38).
} 
Let us now discuss the wall crossing. We first consider the I $\rightarrow$ III crossing. There we have a mixed branch with the unbroken gauge group $G_{1}=\mathrm{U}(1)_{1}$ and broken gauge group $G / G_{1} \cong \mathrm{U}(1)_{2}$. The Coulomb and Higgs parts of the fields are

(C) $X(1,0 \mid 1,0), \quad Z_{1,2,3}(-1,-1 \mid 0,1), \eta_{3}^{1,2}(-1,-1 \mid-1,0), \quad \eta_{1}^{1,2}(1,0 \mid 0,-1)$,

(H) $Y_{1,2,3}(1 \mid 0,0), \quad \eta_{2}^{1,2}(1 \mid-1,-1)$.

Inside the parenthesis are the gauge and the flavor charges, copied from (8.30) except that the $\mathrm{U}(1)_{F_{2}}$ charges are modified so that the Higgs fields $Y_{1,2,3}$ are neutral. For the fields in $(\mathrm{H})$, we omit the $G_{1}=\mathrm{U}(1)_{1}$ charges which are trivial by definition. The charges under $\mathrm{U}(1)_{2}$ are regarded as those under $G / G_{1} \cong \mathrm{U}(1)_{2}$. Let us first analyze the $\sigma_{1}<0$ mixed branch. The equation (3.47) has a unique solution which yields $N_{\text {eff }}=-2$. Thus, there is no supersymmetric ground state for $\zeta^{1}>0$ and a unique supersymmetric ground state of the form (3.52) for $\zeta^{1}<0$ :

$$
|\mathrm{C}-\mathrm{vac}\rangle=\left|\sigma_{1}\right| \mathrm{e}^{-\zeta^{1} \sigma_{1}} \bar{\lambda}_{z_{1}} \bar{\psi}_{z_{2}} \bar{\psi}_{z_{3}} \bar{\eta}_{1}^{1} \bar{\eta}_{1}^{2}|0\rangle_{\mathrm{OSC}} \quad\left(-\frac{1}{2} \mid \frac{1}{2}, \frac{1}{2}\right) .
$$

We listed the charge of the state under $G / G_{1} \times \mathrm{U}(1)_{F_{1}} \times \mathrm{U}(1)_{F_{2}}$, from (3.54), which provides the background charge in the effective theory on the Higgs branch. The Higgs branch theory is thus the $\mathbf{C P}^{2}$ model with Wilson line $\mathrm{q}=-\frac{1}{2}$ and the fermi-multiplet with values in $\mathcal{E}=\mathcal{O}(1)^{\oplus 2}$. Ground states correspond to Dolbeault cohomology classes with values in

$$
\begin{aligned}
\mathfrak{F} & =\sqrt{K}_{\mathbf{C P}^{2}} \otimes \operatorname{det}^{-\frac{1}{2}} \mathcal{E} \otimes \wedge \mathcal{E} \otimes \mathcal{O}\left(-\frac{1}{2}\right)=(\wedge \mathcal{E})(-3) \\
& =\mathcal{O}(-3) \oplus \mathcal{O}(-2)^{\oplus 2} \oplus \mathcal{O}(-1) .
\end{aligned}
$$

There is a unique Higgs vacuum corresponding to $H^{0,2}\left(\mathbf{C P}^{2}, \mathcal{O}(-3)\right) \cong \mathbf{C}$. Since $\eta_{2}^{1,2}$ have $\mathrm{U}(1)_{F_{1}} \times \mathrm{U}(1)_{F_{2}}$ charge $(-1,-1)$, their oscillator vacuum, which corresponds to the term $\mathcal{O}(-3)$ in $(8.42)$, has charge $(1,1)$. Taking the background charge (8.41) into account, we find that the ground state has charge $\left(\frac{3}{2}, \frac{3}{2}\right)$. Note also that it has $(-1)^{F}=1$ if $|0\rangle_{\text {osc }}$ is even. Therefore, it contributes to the change in the index by $+y_{1}^{\frac{3}{2}} y_{2}^{\frac{3}{2}}$. Let us next analyze the $\sigma_{1}>0$ mixed branch. The equation (3.48) has a unique solution with $N_{\text {eff }}=-2$. Thus, there is a supersymmetric ground state only when $\zeta^{1}<0$ which is of the form (3.53):

$$
|\mathrm{C}-\mathrm{vac}\rangle=\left|\sigma_{1}\right| \mathrm{e}^{\zeta^{1} \sigma_{1}} \bar{\psi}_{x} \bar{\eta}_{3}^{1} \bar{\eta}_{3}^{2}|0\rangle_{\mathrm{OSC}} \quad\left(\frac{1}{2} \mid-\frac{1}{2},-\frac{1}{2}\right) .
$$

The Higgs branch theory is the $\mathbf{C P}^{2}$ model with with Wilson line $\mathrm{q}=\frac{1}{2}$ and the fermimultiplet with values in $\mathcal{E}=\mathcal{O}(1)^{\oplus 2}$. Ground states correspond to Dolbeault cohomology classes with values in

$$
\begin{aligned}
\mathfrak{F} & =\sqrt{K}_{\mathbf{C P}^{2}} \otimes \operatorname{det}^{-\frac{1}{2}} \mathcal{E} \otimes \wedge \mathcal{E} \otimes \mathcal{O}\left(\frac{1}{2}\right)=(\wedge \mathcal{E})(-2) \\
& =\mathcal{O}(-2) \oplus \mathcal{O}(-1)^{\oplus 2} \oplus \mathcal{O} .
\end{aligned}
$$

There is a unique Higgs vacuum corresponding to $H^{0,0}\left(\mathbf{C P}^{2}, \mathcal{O}\right) \cong \mathbf{C}$. The state $\bar{\eta}_{2}^{1} \bar{\eta}_{2}^{2}|0\rangle$, which corresponds to the term $\mathcal{O}$ in $(8.44)$, has charge $(-1,-1)$ under $\mathrm{U}(1)_{F_{1}} \times \mathrm{U}(1)_{F_{2}}$. 
Taking the background charge (8.43) into account, we find that the ground state has charge $\left(-\frac{3}{2},-\frac{3}{2}\right)$. Note also that it has $(-1)^{F}=-1$ if $|0\rangle_{\text {osC }}$ is even. Therefore, it contributes to the change in the index by $-y_{1}^{-\frac{3}{2}} y_{2}^{-\frac{3}{2}}$. To summarize, the change in the index for the $\mathrm{I} \rightarrow$ III wall crossing is

$$
\Delta_{\mathrm{I} \rightarrow \mathrm{III}} I=y_{1}^{\frac{3}{2}} y_{2}^{\frac{3}{2}}-y_{1}^{-\frac{3}{2}} y_{2}^{-\frac{3}{2}} .
$$

The II $\rightarrow$ III crossing is similar and we find the same expression for $\Delta I$ as above,

$$
\Delta_{\mathrm{II} \rightarrow \mathrm{III}} I=y_{1}^{\frac{3}{2}} y_{2}^{\frac{3}{2}}-y_{1}^{-\frac{3}{2}} y_{2}^{-\frac{3}{2}}
$$

Finally, let us consider the I $\rightarrow$ II crossing. There we have a mixed branch with the unbroken gauge group $G_{1}=\mathrm{U}(1)_{2}$ and broken gauge group $G / G_{1} \cong \mathrm{U}(1)_{1}$. The Coulomb and the Higgs parts of the fields are

(C) $Y_{1,2,3}(0,1 \mid 0,1), \quad Z_{1,2,3}(-1,-1 \mid 1,0), \quad \eta_{3}^{1,2}(-1,-1 \mid 0,-1), \quad \eta_{2}^{1,2}(0,1 \mid-1,0)$,

(H) $X(1 \mid 0,0), \eta_{1}^{1,2}(1 \mid-1,-1)$.

The gauge and the flavor charges are copied from (8.30) except that the $\mathrm{U}(1)_{F_{1}}$ charges are modified so that the Higgs field $X$ is neutral. For the fields in $(\mathrm{H})$, we omit the $G_{1}=\mathrm{U}(1)_{2}$ charges which are trivial by definition. The charges under $\mathrm{U}(1)_{1}$ are regarded as those under $G / G_{1} \cong \mathrm{U}(1)_{1}$. The supersymmetry equation for the matter sector, (3.47) for $\sigma_{2}<0$ and (3.48) for $\sigma_{2}>0$, has no solution. Thus, there is no supersymmetric ground states in the mixed branch theory for both $\zeta^{2}>0$ and $\zeta^{2}<0$. In particular, the index does not change,

$$
\Delta_{\mathrm{I} \rightarrow \mathrm{II}} I=0 .
$$

The results for the index, (8.36), (8.37) and (8.40), are consistent with the wall crossing formulae, (8.45), (8.46) and (8.47).

\section{Acknowledgments}

We would like to thank Francesco Benini, Jyotirmoy Bhattacharya, Richard Eager, SeungJoo Lee, Yu Nakayama, Tomoki Ohtsuki, Mauricio Romo, Ashoke Sen, Yuji Tachikawa, Zhao-Long Wang, Chris Woodward for discussions, conversations, instructions, collaboration on related works, and encouragement. HK and PY thank Kavli IPMU for the hospitality during their visit. KH and PY are also grateful to organizers of RIKKYO MathPhys 2014, by which time the main results of this work have emerged, for providing a stimulating environment for the collaboration. This work is supported in part by JSPS Grant-in-Aid for Scientific Research No. 21340109 and WPI Initiative, MEXT, Japan at Kavli IPMU, the University of Tokyo. We would like to thank the referee for spotting many mistakes in the manuscript. The paper and our understanding have improved significantly by the revision. We would also like to thank Edward Witten for great help during the revision process. 


\section{A $\quad 1 \mathrm{~d} \mathcal{N}=2$ supersymmetry}

\section{A.1 $1 \mathrm{~d} \mathcal{N}=2$ superspace}

The $\mathcal{N}=2$ superspace has time coordinate $t$ and fermionic coordinates $\theta$ and $\bar{\theta}$. Supersymmetry is

$$
\delta=-\epsilon \mathrm{Q}+\bar{\epsilon} \overline{\mathrm{Q}}
$$

where $\mathrm{Q}$ and $\overline{\mathrm{Q}}$ are differential operators

$$
\mathrm{Q}=\frac{\partial}{\partial \theta}+\frac{i}{2} \bar{\theta} \frac{\partial}{\partial t}, \quad \overline{\mathrm{Q}}=-\frac{\partial}{\partial \bar{\theta}}-\frac{i}{2} \theta \frac{\partial}{\partial t},
$$

which satisfy $\mathrm{Q}^{2}=\overline{\mathrm{Q}}^{2}=0,\{\mathrm{Q}, \overline{\mathrm{Q}}\}=-i \partial_{t}$. They commutes with another set of operators

$$
\mathrm{D}=\frac{\partial}{\partial \theta}-\frac{i}{2} \bar{\theta} \frac{\partial}{\partial t}, \quad \overline{\mathrm{D}}=-\frac{\partial}{\partial \bar{\theta}}+\frac{i}{2} \theta \frac{\partial}{\partial t}
$$

which satisfy $\mathrm{D}^{2}=\overline{\mathrm{D}}^{2}=0$ and $\{\mathrm{D}, \overline{\mathrm{D}}\}=i \partial_{t}$.

A chiral superfield $\Phi$ is a superfield satisfying $\overline{\mathrm{D}} \Phi=0$. It can be expanded as

$$
\Phi=\phi+\theta \psi-\frac{i}{2} \theta \bar{\theta} \dot{\phi}
$$

Supersymmetry variation of the components fields follows from (A.1)

$$
\begin{aligned}
\delta \phi & =-\epsilon \psi, \\
\delta \psi & =i \bar{\epsilon} \dot{\phi} .
\end{aligned}
$$

A fermi superfield $\mathfrak{Y}$ is a fermionic superfield satisfying $\bar{D} \mathfrak{Y}=E(\Phi)$ with $E(\Phi)$ a holomorphic function of a chiral superfield $\Phi$. It is expanded as

$$
\mathfrak{Y}=\eta-\theta F-\frac{i}{2} \theta \bar{\theta} \dot{\eta}-\bar{\theta} E(\Phi) .
$$

Supersymmetry variation of the components is

$$
\begin{aligned}
\delta \eta & =\epsilon F+\bar{\epsilon} E(\phi), \\
\delta F & =\bar{\epsilon}\left(-i \dot{\eta}+\psi^{i} \partial_{i} E(\phi)\right) .
\end{aligned}
$$

For the chiral and fermi superfields as above, we have $(\doteq$ stands for equality up to total derivatives)

$$
\begin{aligned}
& \int \mathrm{d} \theta \mathrm{d} \bar{\theta} i \bar{\Phi} \dot{\Phi} \doteq \dot{\bar{\phi}} \dot{\phi}+i \bar{\psi} \dot{\psi} \\
& \int \mathrm{d} \theta \mathrm{d} \bar{\theta} \overline{\mathfrak{Y}} \mathfrak{Y} \doteq i \bar{\eta} \dot{\eta}+\bar{F} F-|E(\phi)|^{2}-\bar{\eta} \partial_{i} E(\phi) \psi^{i}-\bar{\psi}^{\bar{\imath}} \partial_{\bar{\imath}} \overline{E(\phi)} \eta
\end{aligned}
$$

For a set of fermi and chiral superfields $\mathfrak{Y}^{\alpha}, J_{\alpha}(\Phi)$ with $\overline{\mathrm{D}} \mathfrak{Y}^{\alpha}=E^{\alpha}(\Phi)$ and $E^{\alpha}(\phi) J_{\alpha}(\phi)=0$, the combination $\mathfrak{Y}^{\alpha} J_{\alpha}(\Phi)$ is a fermionic chiral superfield, for which we can take

$$
\int \mathrm{d} \theta\left(\mathfrak{Y}^{\alpha} J_{\alpha}(\Phi)\right)_{\bar{\theta}=0}=-\eta^{\alpha} \partial_{i} J_{\alpha}(\phi) \psi^{i}-F^{\alpha} J_{\alpha}(\phi) .
$$

This is the F-term associated to the superpotential $\mathfrak{W}=\mathfrak{Y}^{\alpha} J_{\alpha}(\Phi)$. 


\section{A.2 Gauge theory}

The vector multiplet consists of a $\mathfrak{g}_{\mathbf{C}}$ and an $i \mathfrak{g}$ valued superfields $\Omega$ and $V^{(-)}$which transform as

$$
\mathrm{e}^{\Omega} \longrightarrow h^{-1} \mathrm{e}^{\Omega} k, \quad i V^{(-)} \longrightarrow k^{-1} i V^{(-)} k+k^{-1} \dot{k},
$$

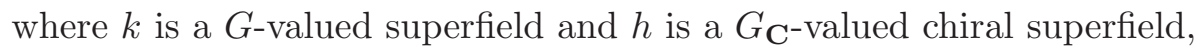

$$
k^{\dagger}=k^{-1}, \quad \overline{\mathrm{D}} h=0 .
$$

One may define the covariant derivatives

$$
\overline{\mathcal{D}}:=\mathrm{e}^{-\Omega} \overline{\mathrm{D}} \mathrm{e}^{\Omega}, \quad \mathcal{D}:=\mathrm{e}^{\Omega^{\dagger}} \mathrm{De}^{-\Omega^{\dagger}}, \quad \mathcal{D}_{t}^{(-)}:=\partial_{t}+i V^{(-)},
$$

which are invariant under the chiral gauge transformation by $h$ but transform covariantly under the unitary gauge transformation by $k$ :

$$
\overline{\mathcal{D}} \rightarrow k^{-1} \overline{\mathcal{D}} k, \quad \mathcal{D} \rightarrow k^{-1} \mathcal{D} k, \quad \mathcal{D}_{t}^{(-)} \rightarrow k^{-1} \mathcal{D}_{t}^{(-)} k .
$$

The first two of them satisfy the relations

$$
\overline{\mathcal{D}}^{2}=\mathcal{D}^{2}=0, \quad\{\mathcal{D}, \overline{\mathcal{D}}\}=i \mathcal{D}_{t}^{(+)}:=i\left(\partial_{t}+i V^{(+)}\right),
$$

for some superfield $V^{(+)}$. We define the field strength superfield by

$$
\Upsilon:=\left[\overline{\mathcal{D}}, \mathcal{D}_{t}^{(-)}\right] .
$$

It is unitary covariant, $\Upsilon \rightarrow k^{-1} \Upsilon k$, and (covariantly) chiral, $\overline{\mathcal{D}} \Upsilon=0$. The gauge kinetic term is

$$
L_{\text {guage }}=\int \mathrm{d} \theta \mathrm{d} \bar{\theta} \frac{1}{2 e^{2}} \operatorname{Tr} \bar{\Upsilon} \Upsilon .
$$

For a $G$-invariant linear form $\zeta: i \mathfrak{g} \rightarrow \mathbf{R}$, we have the FI term

$$
L_{\mathrm{FI}}=\int \mathrm{d} \theta \mathrm{d} \bar{\theta} \zeta\left(V^{(-)}\right) .
$$

For unitary covariant chiral and fermi superfields $\Phi$ and $\mathfrak{Y}$, the kinetic terms are

$$
L_{\text {chiral }}=\int \mathrm{d} \theta \mathrm{d} \bar{\theta} i \bar{\Phi} \mathcal{D}_{t}^{(-)} \Phi, \quad L_{\text {fermi }}=\int \mathrm{d} \theta \mathrm{d} \bar{\theta} \overline{\mathfrak{Y}} \mathfrak{Y} .
$$

Component expressions. Let us define the Wess-Zumino gauge as

$$
\Omega=-\frac{1}{2} \theta \bar{\theta}\left(v_{t}+\sigma\right) .
$$

The residual gauge symmetry is

$$
k=g, \quad h=g-\frac{i}{2} \theta \bar{\theta} \dot{g},
$$

for a $G$-valued function $g$ of $t$. For this choice, we have

$$
\overline{\mathcal{D}}=-\partial_{\bar{\theta}}+\frac{i}{2} \theta D_{t}^{(+)}, \quad \mathcal{D}=\partial_{\theta}-\frac{i}{2} \bar{\theta} D_{t}^{(+)} .
$$


where

$$
D_{t}^{( \pm)}:=D_{t} \pm i \sigma=\partial_{t}+i\left(v_{t} \pm \sigma\right) .
$$

We may write

$$
\begin{aligned}
V^{(-)} & =v_{t}-\sigma-i \theta \bar{\lambda}-i \bar{\theta} \lambda+\theta \bar{\theta} D, \\
\Phi & =\phi+\theta \psi-\frac{i}{2} \theta \bar{\theta} D_{t}^{(+)} \phi, \\
\mathfrak{Y} & =\eta-\theta F-\frac{i}{2} \theta \bar{\theta} D_{t}^{(+)} \psi_{-}-\bar{\theta} E(\Phi) .
\end{aligned}
$$

They transform covariantly under the residual gauge transformations (A.21) except $i v_{t} \rightarrow$ $g^{-1} i v_{t} g+g^{-1} \dot{g}$. The field strength superfields is then written as

$$
\Upsilon=-\lambda+\theta\left(D_{t} \sigma+i D\right)+\frac{i}{2} \theta \bar{\theta} D_{t}^{(+)} \lambda_{-} .
$$

Under the supersymmetry $\delta=-\epsilon \mathrm{Q}+\bar{\epsilon} \overline{\mathrm{Q}}$, the Wess-Zumino gauge is not preserved. To bring things back to the Wess-Zumino gauge, we need to perform the following unitary and chiral gauge transforms (to the first order):

$$
k=1+\theta \bar{\epsilon} \frac{v_{t}+\sigma}{2}+\bar{\theta} \epsilon \frac{v_{t}+\sigma}{2}, \quad h=1+\theta \bar{\epsilon}\left(v_{t}+\sigma\right) .
$$

This way, we find the supersymmetry transform of the component fields:

$$
\begin{aligned}
\delta v_{t} & =-\delta \sigma=\frac{i}{2} \epsilon \bar{\lambda}+\frac{i}{2} \bar{\epsilon} \lambda, \\
\delta \lambda & =\epsilon\left(D_{t} \sigma+i D\right), \\
\delta D & =\frac{1}{2} \epsilon D_{t}^{(+)} \bar{\lambda}-\frac{1}{2} \bar{\epsilon} D_{t}^{(+)} \lambda, \\
\delta \phi & =-\epsilon \psi, \\
\delta \psi & =i \bar{\epsilon} D_{t}^{(+)} \phi \\
\delta \eta & =\epsilon F+\bar{\epsilon} E(\phi), \\
\delta F & =\bar{\epsilon}\left(-i D_{t}^{(+)} \eta+\psi^{i} \partial_{i} E(\phi)\right) .
\end{aligned}
$$

The supersymmetric Lagrangians have the following expressions:

$$
\begin{aligned}
L_{\text {gauge }} & \doteq \frac{1}{2 e^{2}} \operatorname{Tr}\left[\left(D_{t} \sigma\right)^{2}+i \bar{\lambda} D_{t}^{(+)} \lambda+D^{2}\right], \\
L_{\mathrm{FI}} & =-\zeta(D), \\
L_{\text {chiral }} & \doteq D_{t} \bar{\phi} D_{t} \phi+i \bar{\psi} D_{t}^{(-)} \psi+\bar{\phi}\left\{D-\sigma^{2}\right\} \phi-i \bar{\phi} \lambda \psi+i \overline{\psi \lambda} \phi, \\
L_{\text {fermi }} & \doteq i \bar{\eta} D_{t}^{(+)} \eta+\bar{F} F-\overline{E(\phi)} E(\phi)-\bar{\eta} \partial_{i} E(\phi) \psi^{i}-\bar{\psi}^{\imath} \partial_{\bar{\imath}} \overline{E(\phi)} \eta
\end{aligned}
$$




\section{A.3 Non-linear sigma model}

We consider a Kähler manifold $(X, g)$ and a holomorphic vector bundle with a hermitian metric $(\mathcal{E}, h)$ on $X$. Locally, the Kähler metric is written using a Kähler potential as $g_{i \bar{\jmath}}=\partial_{i} \partial_{\bar{\jmath}} K$, and the fiber metric $h$ is expressed as a hermitian matrix $h_{\alpha \bar{\beta}}=h\left(e_{\beta}, e_{\alpha}\right)$ for a choice of local holomorphic frame $\left\{e_{\alpha}\right\}$. The variables are $X$-valued chiral superfield $\Phi$ and $\mathcal{E}$-valued chiral fermi superfield $\mathfrak{Y}$. Their kinetic terms are

$$
\begin{gathered}
\int \mathrm{d} \theta \mathrm{d} \bar{\theta} i \partial_{i} K(\Phi, \bar{\Phi}) \dot{\Phi}^{i} \doteq g_{i \bar{\jmath}} \dot{\bar{\phi}}^{\bar{\phi}} \dot{\phi}^{i}+i g_{i \bar{\jmath}} \bar{\psi}^{\bar{\jmath}} D_{t} \psi^{i} \\
\int \mathrm{d} \theta \mathrm{d} \bar{\theta} h_{\alpha \bar{\beta}}(\Phi, \bar{\Phi}) \bar{\Xi}_{-}^{\bar{\beta}} \mathfrak{Y}_{-}^{\alpha} \doteq i h_{\alpha \bar{\beta}} \bar{\eta}^{\bar{\beta}} D_{t} \eta^{\alpha}+h_{\alpha \bar{\beta}} \bar{\eta}^{\bar{\beta}} F_{i \bar{\jmath} \gamma}^{\alpha} \psi^{i} \bar{\psi}^{\bar{\jmath}} \eta^{\gamma}+h_{\alpha \bar{\beta}} \overline{\widetilde{F}}^{\bar{\beta}} \widetilde{F}^{\alpha}
\end{gathered}
$$

where

$$
D_{t} \psi^{i}=\dot{\psi}^{i}+\dot{\phi}^{j} \Gamma_{j k}^{i} \psi^{k}, \quad D_{t} \eta^{\alpha}=\dot{\eta}^{\alpha}+\dot{\phi}^{j} A_{j \beta}^{\alpha} \eta^{\beta}
$$

with $\Gamma_{j k}^{i}:=g^{i \bar{l}} \partial_{j} g_{k \bar{l}}$ and $A_{j \beta}^{\alpha}:=h^{\alpha \bar{\gamma}} \partial_{j} h_{\beta \bar{\gamma}}$ which are the connection forms of $T_{X}$ and $\mathcal{E}$ in the holomorphic frames. Also, $F_{i \bar{\jmath}}^{\alpha} \beta$ is the curvature of $\mathcal{E}$,

$$
F_{i \bar{\jmath} \beta}^{\alpha}:=\left(\partial_{i} A_{\bar{\jmath}}-\partial_{\bar{\jmath}} A_{i}+\left[A_{i}, A_{\bar{\jmath}}\right]\right)_{\beta}^{\alpha}=-h^{\alpha \bar{\gamma}}\left(\partial_{i} \partial_{\bar{\jmath}} h_{\beta \bar{\gamma}}-\partial_{\bar{\jmath}} h_{\delta \bar{\gamma}} h^{\delta \bar{\alpha}} \partial_{i} h_{\beta \bar{\alpha}}\right)
$$

and $\widetilde{F}^{\alpha}$ are the covariant auxiliary fields,

$$
\widetilde{F}^{\alpha}:=F^{\alpha}-\psi^{j} A_{j \beta}^{\alpha} \eta^{\beta} .
$$

$\widetilde{F}^{\alpha}$ 's are covariant because $F^{\alpha}$ 's transform inhomogeneously under the holomorphic frame change $e_{\beta^{\prime}}=e_{\alpha} g_{\beta^{\prime}}^{\alpha}$ as $F^{\alpha}=g_{\beta^{\prime}}^{\alpha} F^{\beta^{\prime}}-\psi^{i} \partial_{i} g_{\beta^{\prime}}^{\alpha} \eta^{\beta^{\prime}}$, which follows from $\mathfrak{Y}^{\alpha}=g_{\beta^{\prime}}^{\alpha}(\Phi) \mathfrak{Y}^{\beta^{\prime}}$.

\section{A.4 $\mathcal{N}=4$ theories}

An $\mathcal{N}=4$ vector multiplet consists of an $\mathcal{N}=2$ vector multiplet $\left(\Omega, V^{(-)}\right)$and an $\mathcal{N}=2$ chiral multiplet $\Sigma$ in the adjoint representation. An $\mathcal{N}=4$ chiral multiplet consists of $\mathcal{N}=2$ chiral and fermi multiplets in a representation, $\Phi$ and $\mathfrak{Y}$, which obey $\overline{\mathcal{D}} \mathfrak{Y}=\Sigma \Phi$. An $\mathcal{N}=4$ supersymmetric Lagrangians are

$$
\begin{aligned}
\boldsymbol{L}_{\text {gauge }} & =\int \mathrm{d} \theta \mathrm{d} \bar{\theta} \frac{1}{2 e^{2}} \operatorname{Tr}\left[i \bar{\Sigma} \mathcal{D}_{t}^{(-)} \Sigma+\bar{\Upsilon} \Upsilon\right], \\
\boldsymbol{L}_{\text {chiral }} & =\int \mathrm{d} \theta \mathrm{d} \bar{\theta}\left[i \bar{\Phi} \mathcal{D}_{t}^{(-)} \Phi+\overline{\mathfrak{Y}} \mathfrak{Y}\right], \\
\boldsymbol{L}_{W} & =-\left.\operatorname{Re} \int \mathrm{d} \theta \mathfrak{Y}^{i} \partial_{i} W(\Phi)\right|_{\bar{\theta}=0},
\end{aligned}
$$

where $W(\Phi)$ is a gauge invariant and holomorphic function of $\Phi$. They are the dimensional reductions of the gauge kinetic term, matter kinetic term, and superpotential term, respectively, in $4 \mathrm{~d} \mathcal{N}=1$ or $2 \mathrm{~d}(2,2)$ gauge theories. The FI term $(2.7)$ is also $\mathcal{N}=4$ supersymmetric. 
Open Access. This article is distributed under the terms of the Creative Commons Attribution License (CC-BY 4.0), which permits any use, distribution and reproduction in any medium, provided the original author(s) and source are credited.

\section{References}

[1] E. Witten, Constraints on supersymmetry breaking, Nucl. Phys. B 202 (1982) 253 [INSPIRE].

[2] E. Witten, Phases of $N=2$ theories in two-dimensions, Nucl. Phys. B 403 (1993) 159 [hep-th/9301042] [INSPIRE].

[3] K. Intriligator and N. Seiberg, Aspects of $3 d N=2$ Chern-Simons-matter theories, JHEP 07 (2013) 079 [arXiv: 1305.1633] [INSPIRE].

[4] E. Witten, Bound states of strings and p-branes, Nucl. Phys. B 460 (1996) 335 [hep-th/9510135] [INSPIRE].

[5] M.R. Douglas and G.W. Moore, D-branes, quivers and ALE instantons, hep-th/9603167 [INSPIRE].

[6] M.R. Douglas, Gauge fields and D-branes, J. Geom. Phys. 28 (1998) 255 [hep-th/9604198] [INSPIRE].

[7] M.R. Douglas, B.R. Greene and D.R. Morrison, Orbifold resolution by D-branes, Nucl. Phys. B 506 (1997) 84 [hep-th/9704151] [INSPIRE].

[8] P. Yi, Witten index and threshold bound states of D-branes, Nucl. Phys. B 505 (1997) 307 [hep-th/9704098] [INSPIRE].

[9] S. Sethi and M. Stern, D-brane bound states redux, Commun. Math. Phys. 194 (1998) 675 [hep-th/9705046] [INSPIRE].

[10] S. Cecotti, P. Fendley, K.A. Intriligator and C. Vafa, A new supersymmetric index, Nucl. Phys. B 386 (1992) 405 [hep-th/9204102] [INSPIRE].

[11] N. Seiberg and E. Witten, Electric-magnetic duality, monopole condensation and confinement in $N=2$ supersymmetric Yang-Mills theory, Nucl. Phys. B 426 (1994) 19 [Erratum ibid. B 430 (1994) 485] [hep-th/9407087] [INSPIRE].

[12] N. Seiberg and E. Witten, Monopoles, duality and chiral symmetry breaking in $N=2$ supersymmetric QCD, Nucl. Phys. B 431 (1994) 484 [hep-th/9408099] [INSPIRE].

[13] F. Ferrari and A. Bilal, The strong coupling spectrum of the Seiberg-Witten theory, Nucl. Phys. B 469 (1996) 387 [hep-th/9602082] [INSPIRE].

[14] O. Bergman, Three pronged strings and $1 / 4$ BPS states in $N=4$ super Yang-Mills theory, Nucl. Phys. B 525 (1998) 104 [hep-th/9712211] [INSPIRE].

[15] K.-M. Lee and P. Yi, Dyons in $N=4$ supersymmetric theories and three pronged strings, Phys. Rev. D 58 (1998) 066005 [hep-th/9804174] [INSPIRE].

[16] D. Bak, C.-K. Lee, K.-M. Lee and P. Yi, Low-energy dynamics for $1 / 4$ BPS dyons, Phys. Rev. D 61 (2000) 025001 [hep-th/9906119] [inSPIRE].

[17] J.P. Gauntlett, N. Kim, J. Park and P. Yi, Monopole dynamics and BPS dyons $N=2$ super Yang-Mills theories, Phys. Rev. D 61 (2000) 125012 [hep-th/9912082] [INSPIRE].

[18] M. Stern and P. Yi, Counting Yang-Mills dyons with index theorems, Phys. Rev. D 62 (2000) 125006 [hep-th/0005275] [INSPIRE]. 
[19] F. Denef, Supergravity flows and D-brane stability, JHEP 08 (2000) 050 [hep-th/0005049] [INSPIRE].

[20] M.R. Douglas, D-branes, categories and $N=1$ supersymmetry, J. Math. Phys. 42 (2001) 2818 [hep-th/0011017] [InSPIRE].

[21] S. Kachru and J. McGreevy, Supersymmetric three cycles and supersymmetry breaking, Phys. Rev. D 61 (2000) 026001 [hep-th/9908135] [INSPIRE].

[22] F. Denef, Quantum quivers and Hall/hole halos, JHEP 10 (2002) 023 [hep-th/0206072] [INSPIRE].

[23] F. Denef and G.W. Moore, Split states, entropy enigmas, holes and halos, JHEP 11 (2011) 129 [hep-th/0702146] [INSPIRE].

[24] M. Reineke, The Harder-Narasimhan system in quantum groups and cohomology of quiver moduli, Invent. Math. 152 (2003) 349 [math/0204059].

[25] J. de Boer, S. El-Showk, I. Messamah and D. Van den Bleeken, Quantizing $N=2$ multicenter solutions, JHEP 05 (2009) 002 [arXiv:0807.4556] [INSPIRE].

[26] J. Manschot, B. Pioline and A. Sen, Wall crossing from Boltzmann black hole halos, JHEP 07 (2011) 059 [arXiv:1011.1258] [INSPIRE].

[27] S. Lee and P. Yi, Framed BPS states, moduli dynamics and wall-crossing, JHEP 04 (2011) 098 [arXiv: 1102.1729] [INSPIRE].

[28] H. Kim, J. Park, Z. Wang and P. Yi, Ab initio wall-crossing, JHEP 09 (2011) 079 [arXiv: 1107.0723] [INSPIRE].

[29] J. Manschot, B. Pioline and A. Sen, A fixed point formula for the index of multi-centered $N=2$ black holes, JHEP 05 (2011) 057 [arXiv:1103.1887] [INSPIRE].

[30] S.-J. Lee, Z.-L. Wang and P. Yi, Quiver invariants from intrinsic Higgs states, JHEP 07 (2012) 169 [arXiv:1205.6511] [INSPIRE].

[31] S.-J. Lee, Z.-L. Wang and P. Yi, BPS states, refined indices and quiver invariants, JHEP 10 (2012) 094 [arXiv: 1207.0821] [INSPIRE].

[32] J. Manschot, B. Pioline and A. Sen, From black holes to quivers, JHEP 11 (2012) 023 [arXiv: 1207.2230] [INSPIRE].

[33] S.-J. Lee, Z.-L. Wang and P. Yi, Abelianization of BPS quivers and the refined Higgs index, JHEP 02 (2014) 047 [arXiv:1310.1265] [INSPIRE].

[34] A. Sen, Equivalence of three wall-crossing formulae, Commun. Num. Theor. Phys. 6 (2012) 601 [arXiv:1112.2515] [INSPIRE].

[35] M. Kontsevich and Y. Soibelman, Stability structures, motivic Donaldson-Thomas invariants and cluster transformations, arXiv:0811.2435 [INSPIRE].

[36] D. Gaiotto, G.W. Moore and A. Neitzke, Four-dimensional wall-crossing via three-dimensional field theory, Commun. Math. Phys. 299 (2010) 163 [arXiv:0807.4723] [INSPIRE].

[37] D. Gaiotto, G.W. Moore and A. Neitzke, Wall-crossing, Hitchin systems and the WKB approximation, arXiv:0907.3987 [INSPIRE].

[38] I. Bena, M. Berkooz, J. de Boer, S. El-Showk and D. Van den Bleeken, Scaling BPS solutions and pure-Higgs states, JHEP 11 (2012) 171 [arXiv:1205.5023] [INSPIRE]. 
[39] F. Benini, R. Eager, K. Hori and Y. Tachikawa, Elliptic genera of two-dimensional $N=2$ gauge theories with rank-one gauge groups, Lett. Math. Phys. 104 (2014) 465 [arXiv: 1305.0533] [INSPIRE].

[40] F. Benini, R. Eager, K. Hori and Y. Tachikawa, Elliptic genera of $2 d N=2$ gauge theories, arXiv: 1308.4896 [INSPIRE].

[41] K. Hori and D. Tong, Aspects of non-Abelian gauge dynamics in two-dimensional $N=(2,2)$ theories, JHEP 05 (2007) 079 [hep-th/0609032] [INSPIRE].

[42] K. Hori, Duality in two-dimensional $(2,2)$ supersymmetric non-Abelian gauge theories, JHEP 10 (2013) 121 [arXiv:1104.2853] [InSPIRE].

[43] L.C. Jeffrey and F.C. Kirwan, Localization for non-Abelian group actions, Topology 34 (1995) 291 [alg-geom/9307001].

[44] M. Brion and M. Vergne, Arrangement of hyperplanes I: rational functions and Jeffrey-Kirwan residue, Ann. Sci. ENS 32 (1999) 715 [math/9903178].

[45] A. Szenes and M. Vergne, Toric reduction and a conjecture of Batyrev and Materov, Invent. Math. 158 (2004) 453 [math/0306311].

[46] K. Hori, $1 d$ index and wall-crossing, talk at StringMath 2014, Alberta Canada June 2014.

[47] P. Yi, Index theorems for $d=1$ GLSM and BPS states, in Recent Development in Theoretical Physics, Seoul South Korea June 2014.

[48] C. Cordova and S.-H. Shao, An index formula for supersymmetric quantum mechanics, arXiv: 1406.7853 [INSPIRE].

[49] C. Hwang, J. Kim, S. Kim and J. Park, General instanton counting and 5d SCFT, arXiv: 1406.6793 [INSPIRE].

[50] M. Herbst, K. Hori and D. Page, Phases of $N=2$ theories in $1+1$ dimensions with boundary, arXiv:0803.2045 [INSPIRE].

[51] J. Wess and J. Bagger, Supersymmetry and supergravity, Princeton Univ. Pr., Princeton U.S.A. (1992).

[52] K. Hori and J. Knapp, Linear $\sigma$-models with strongly coupled phases - one parameter models, JHEP 11 (2013) 070 [arXiv: 1308.6265] [INSPIRE].

[53] L. Álvarez-Gaumé, Supersymmetry and the Atiyah-Singer index theorem, Commun. Math. Phys. 90 (1983) 161 [inSPIRE].

[54] D. Friedan and P. Windey, Supersymmetric derivation of the Atiyah-Singer index and the chiral anomaly, Nucl. Phys. B 235 (1984) 395 [INSPIRE].

[55] K.A. Intriligator and C. Vafa, Landau-Ginzburg orbifolds, Nucl. Phys. B 339 (1990) 95 [INSPIRE].

[56] H. Jockers, V. Kumar, J.M. Lapan, D.R. Morrison and M. Romo, Non-Abelian $2 D$ gauge theories for determinantal Calabi-Yau varieties, JHEP 11 (2012) 166 [arXiv:1205.3192] [INSPIRE].

[57] D.R. Morrison and M.R. Plesser, Summing the instantons: quantum cohomology and mirror symmetry in toric varieties, Nucl. Phys. B 440 (1995) 279 [hep-th/9412236] [INSPIRE].

[58] I. Brunner, K. Hori, K. Hosomichi and J. Walcher, Orientifolds of Gepner models, JHEP 02 (2007) 001 [hep-th/0401137] [INSPIRE]. 
[59] D. Gaiotto, G.W. Moore and A. Neitzke, Framed BPS states, Adv. Theor. Math. Phys. 17 (2013) 241 [arXiv: 1006.0146] [InSPIRE].

[60] J. Manschot, B. Pioline and A. Sen, On the Coulomb and Higgs branch formulae for multi-centered black holes and quiver invariants, JHEP 05 (2013) 166 [arXiv:1302.5498] [INSPIRE].

[61] J. Manschot, B. Pioline and A. Sen, The Coulomb branch formula for quiver moduli spaces, arXiv: 1404.7154 [INSPIRE].

[62] A. Sen, Arithmetic of quantum entropy function, JHEP 08 (2009) 068 [arXiv:0903.1477] [INSPIRE].

[63] S. Kachru and E. Witten, Computing the complete massless spectrum of a Landau-Ginzburg orbifold, Nucl. Phys. B 407 (1993) 637 [hep-th/9307038] [INSPIRE].

[64] J. Distler and S. Kachru, (0,2) Landau-Ginzburg theory, Nucl. Phys. B 413 (1994) 213 [hep-th/9309110] [INSPIRE].

[65] A. Borel and A. Weil, Représentations linéaires et espaces homogènes Kählériens des groupes de Lie compacts (in French), presented by J-P. Serre, Séminaire Bourbaki, France (1954).

[66] R. Bott, Homogeneous vector bundles, Ann. Math. 66 (1957) 203. 